\title{
MODELING, SIMULATION, AND OPTIMIZATION OF ADVANCED OXIDATION PROCESSES FOR TREATMENT OF POLYMERIC WASTEWATER
}

by

\section{Samira Ghafoori}

BSc, University of Tehran, Tehran, Iran, 2003

MSc, Sharif University of Technology, Tehran, Iran, 2007

\author{
A Dissertation \\ Presented to the School of Graduate Studies at \\ Ryerson University \\ in partial fulfilment of the \\ requirements for the degree of \\ Doctor of Philosophy \\ in the Program of Chemical Engineering
}

Toronto, Ontario, Canada, 2013

(C) Samira Ghafoori, 2013 


\section{Table of Contents}

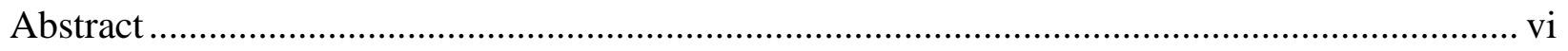

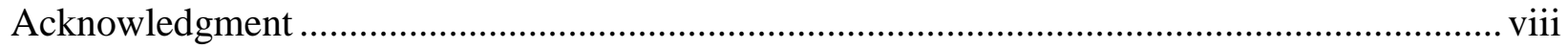

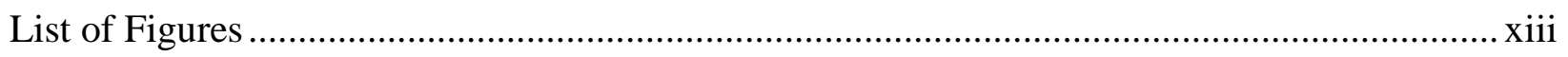

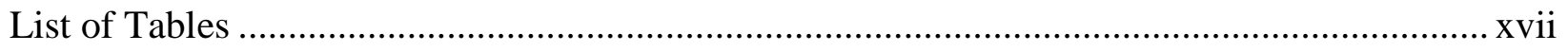

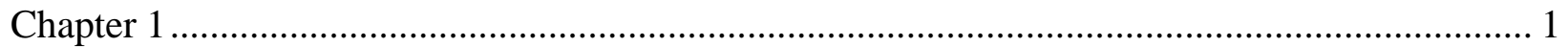

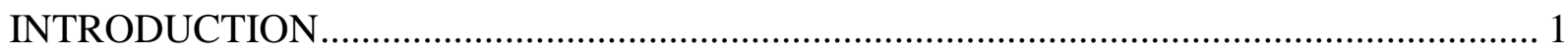

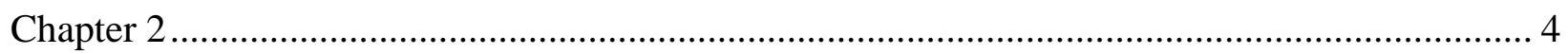

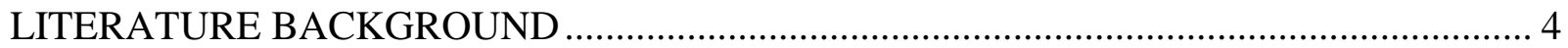

2.1. ADVANCED OXIDATION TECHNOLOGIES (AOTs) ………................................. 4

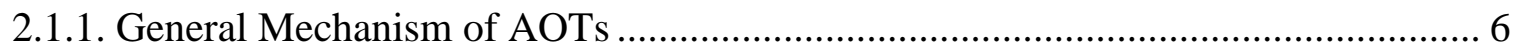

2.1.2. Kinetic Modeling of AOTs ............................................................................. 15

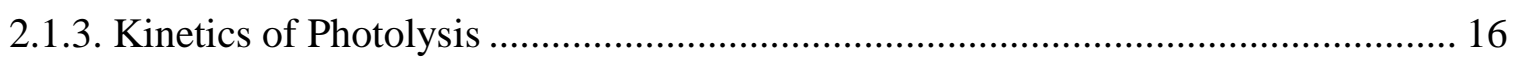

2.2. PHOTOCHEMICAL DEGRADATION OF POLYMERS ……………………............. 22

2.2.1. General Mechanism of Photodegradation of Polymers ........................................... 23

2.2.2. Types of Polymer Chain Scission ..................................................................... 26

2.2.3. Kinetic Modeling of Polymer Degradation........................................................... 28

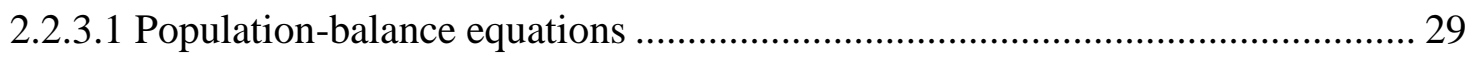

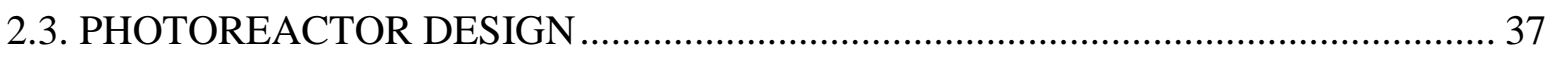

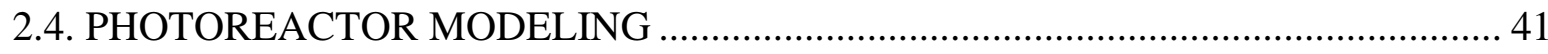

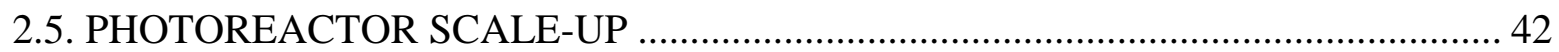

2.6. SYNTHETIC WATER-SOLUBLE POLYMERS AS MODEL COMPOUNDS IN

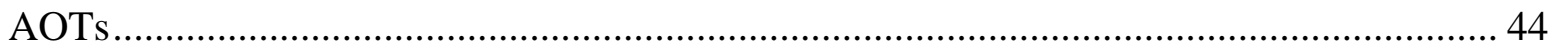

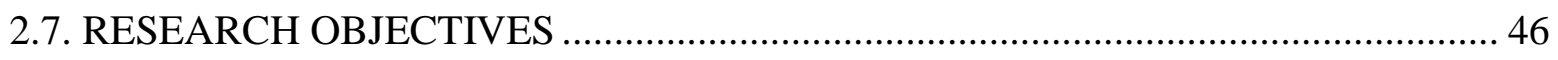

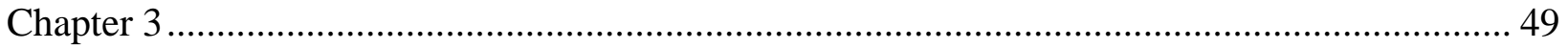

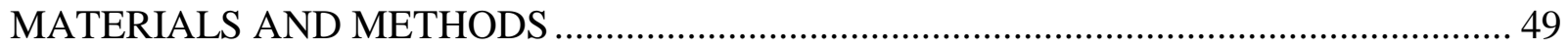

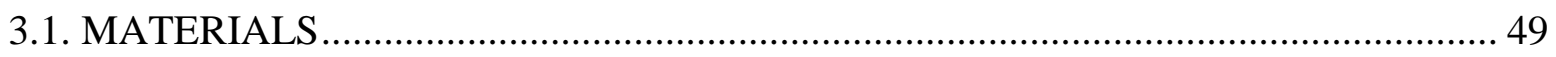

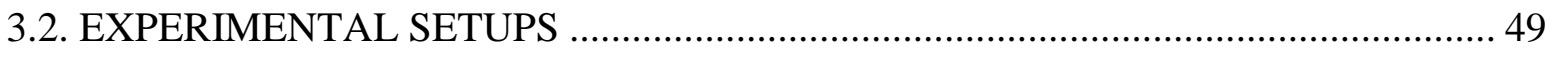

3.2.1. Laboratory-Scale Photoreactor ........................................................................... 50 


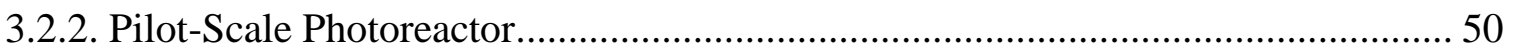

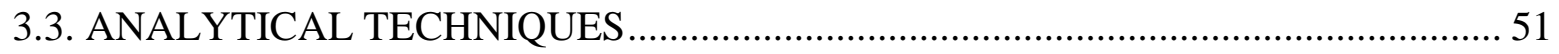

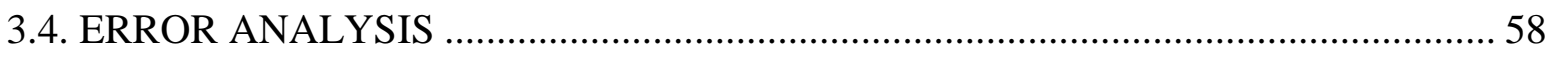

3.5. MODELING, STATISTICAL ANALYSIS, AND OPTIMIZATION ........................... 58

3.5.1. Response Surface Methodology …………………...................................... 58

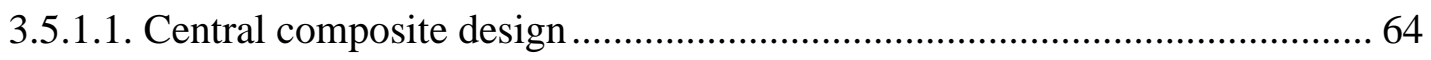

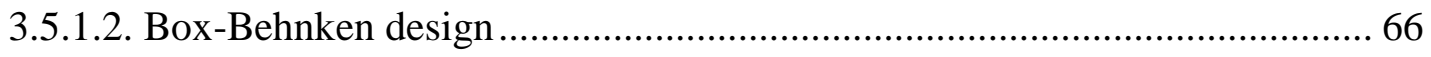

3.5.2. Computational Fluid Dynamics ..................................................................... 68

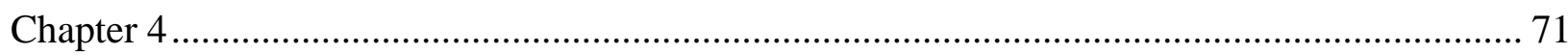

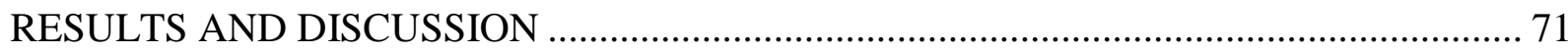

4.1. KINETIC STUDY OF PHOTODEGRADATION OF WATER-SOLUBLE

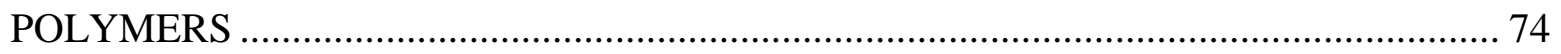

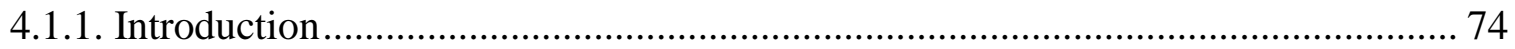

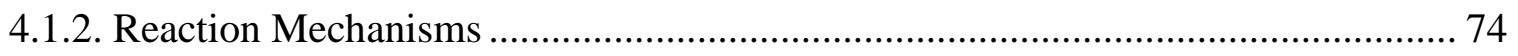

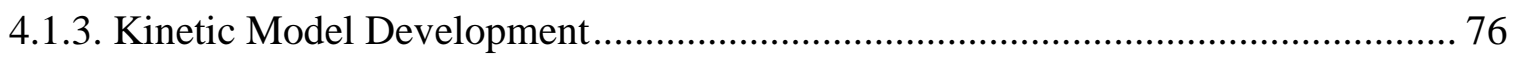

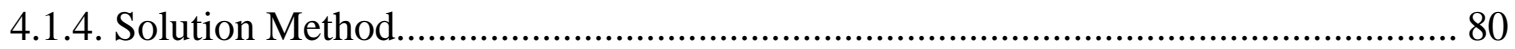

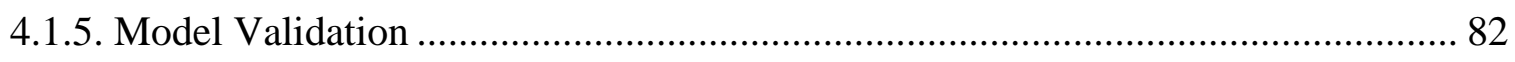

4.1.6. Kinetic Parameter Estimation ............................................................................. 87

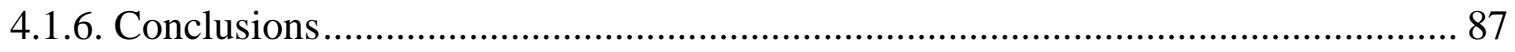

4.2. FREE RADICAL-INDUCED DEGRADATION OF AQUEOUS POLYETHYLENE OXIDE BY UV $/ \mathrm{H}_{2} \mathrm{O}_{2}$ : EXPERIMENTAL DESIGN, REACTION MECHANISM, AND

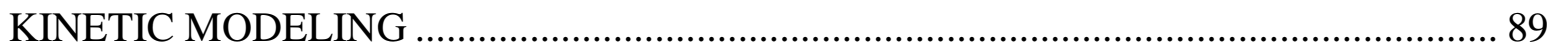

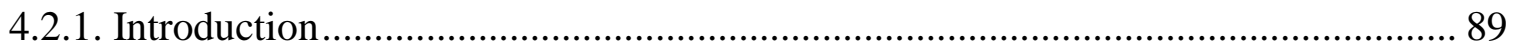

4.2.2. Experimental Procedure .................................................................................... 90

4.2.3. Preliminary Studies ..................................................................................... 90

4.2.4. Experimental Design using Central Composite Design............................................ 91

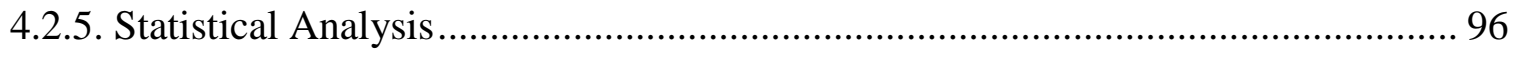

4.2.6. Interaction Effects between the Independent Variables......................................... 96

4.2.7. Optimization of the Process Parameters ................................................................. 99

4.2.8. Kinetic Model Development and Photoreactor Modeling ..................................... 101

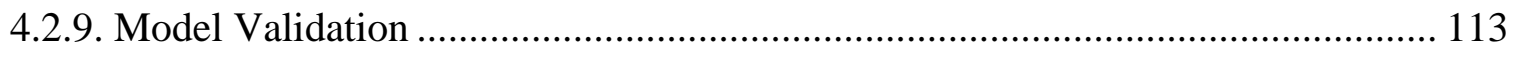

4.2.10. Kinetic Parameter Estimation ....................................................................... 117 
4.2.11. Conclusions 121

4.3. PHOTOASSISTED FENTON-LIKE DEGRADATION OF AQUEOUS POLYACRYLIC ACID: EXPERIMENTAL DESIGN, MECHANISTIC KINETIC

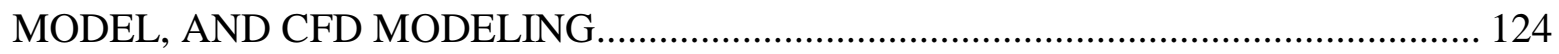

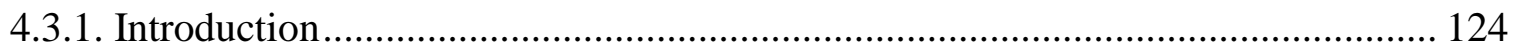

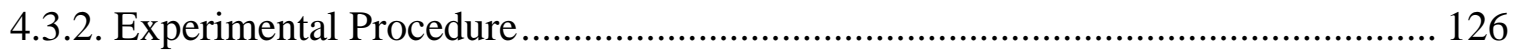

4.3.3. Experimental Design using Box-Behnken Design ............................................. 126

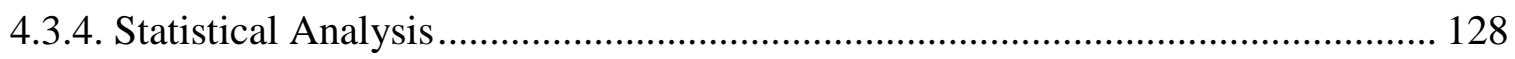

4.3.5. Interaction Effects between the Independent Variables...................................... 132

4.3.6. Optimization of Process Parameters ................................................................... 132

4.3.7. Mechanistic Kinetic Model.......................................................................... 133

4.3.8. Model Validation .............................................................................................. 143

4.3.9. Sensitivity Analysis ......................................................................................... 144

4.3.10. CFD Model Development......................................................................... 150

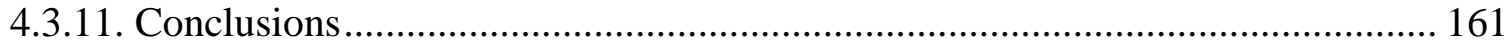

4.4. OPTIMIZATION OF PHOTO-FENTON-LIKE DEGRADATION OF AQUEOUS POLYACRYLIC ACID USING TWO PHOTOREACTORS IN SERIES .......................... 163

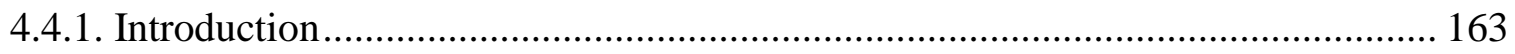

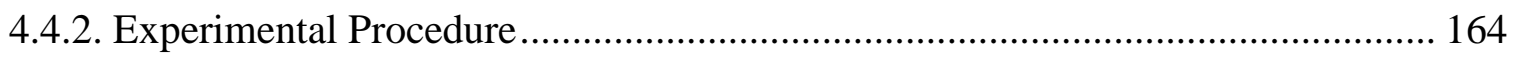

4.4.3. Preliminary Experiments ............................................................................. 164

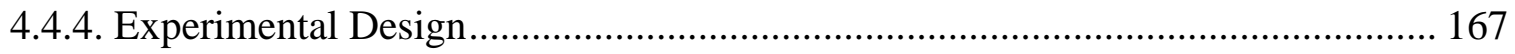

4.4.5. RSM Model Development ............................................................................. 168

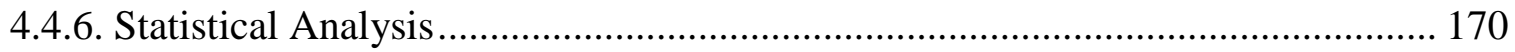

4.4.7. Effect of Model Parameters and their Interactions ............................................. 171

4.4.8. 3D Response Surface and 2D Contour Plots ...................................................... 176

4.4.9. Optimization of the Process Parameters .............................................................. 182

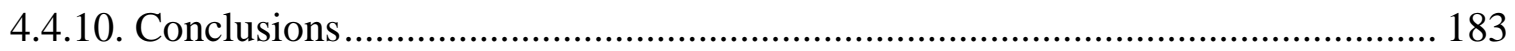

4.5. PHOTOREACTOR SCALE-UP FOR DEGRADATION OF POLYVINYL ALCOHOL IN AQUEOUS SOLUTION USING UV/ $\mathrm{H}_{2} \mathrm{O}_{2}$ PROCESS .......................... 185

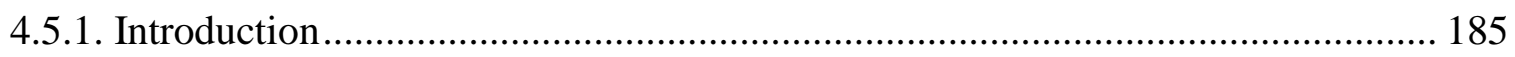

4.5.2. Photoreactor Scale-up Methodology ……………………………………….... 186

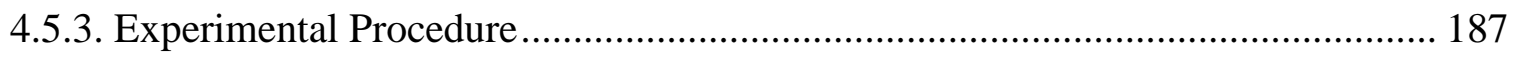


4.5.4. Modeling of the Laboratory-Scale Photoreactor................................................. 187

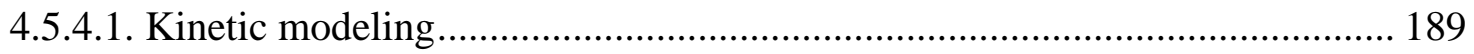

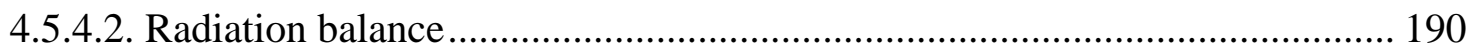

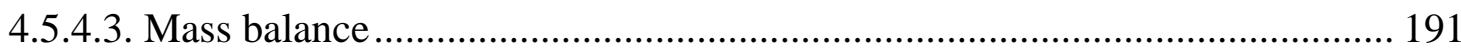

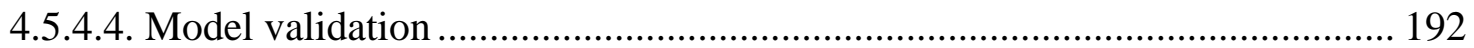

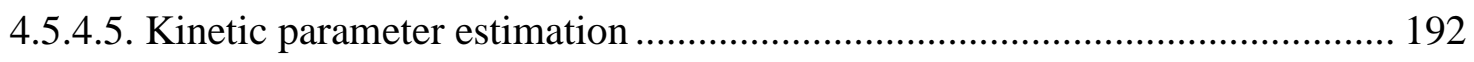

4.5.5. CFD Modeling of the Pilot-Scale Photoreactor ..................................................... 193

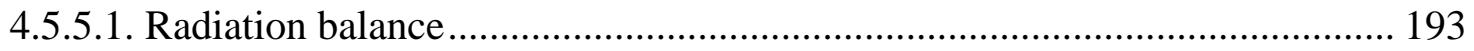

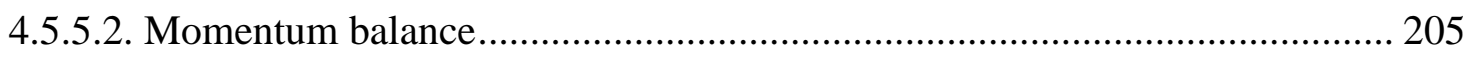

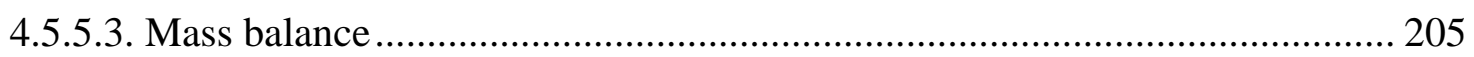

4.5.6. Validation of the Scaling-up Methodology........................................................ 208

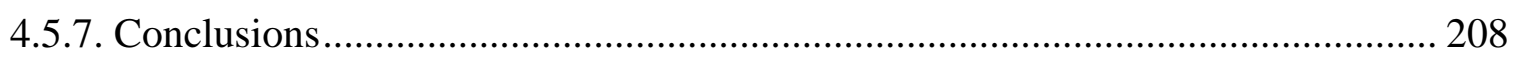

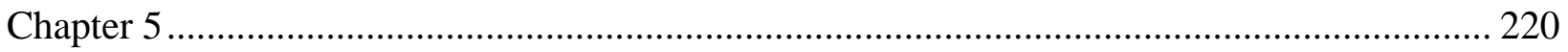

CONCLUSIONS AND RECOMMENDATIONS ……………...................................... 220

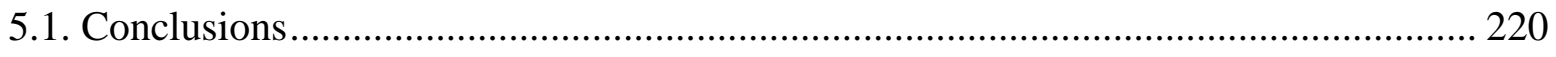

5.2. Recommendations for Future Work....................................................................... 224

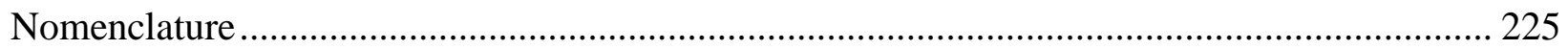

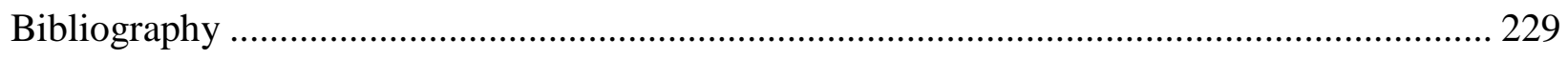

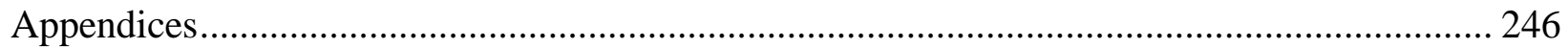

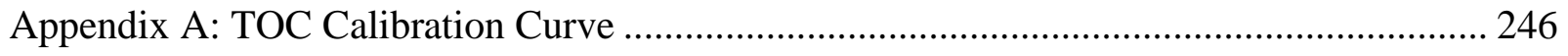

Appendix B: Computer Program ………………………............................................ 250 
I hereby declare that I am the sole author of this dissertation.

I authorize Ryerson University to lend this dissertation to other institutions or individuals for the purpose of scholarly research.

\section{$\underline{\text { Samira Ghafoori }}$}

I further authorize Ryerson University to reproduce this dissertation by photocopying or by other means, in total or in part, at the request of other institutions or individuals for the purpose of scholarly research.

$\underline{\text { Samira Ghafoori }}$ 


\section{Abstract}

\section{Modeling, Simulation, and Optimization of Advanced Oxidation Technologies for Treatment of Polymeric Wastewater}

(C)Samira Ghafoori, 2013

Doctor of Philosophy

Chemical Engineering

Ryerson University

Most synthetic water-soluble polymers from industrial effluents are discarded into the aqueous environment. Due to their water solubility they can reach conventional sewage disposal systems and contaminate water resources as they are not amenable to biodegradation. Therefore, the possibility of rendering these materials to biologically managable chemical compounds is a challenging task.

The applications of advanced oxidation technologies (AOTs) for treating wastewater containing recalcitrant and inhibitory organics have risen drastically during the past few decades. AOTs mediate fast degradation or decomposition of the target organic compounds by producing free radical species.

In this study, the photooxidative degradation of water-soluble polymers in wastewater is studied using different methods of AOTs. The experimental design, statistical analysis, and optimization of different processes of AOTs are investigated employing various methods of response surface methodology (RSM). Using RSM, the effects of different process parameters on the response function are determined. Also, a detailed mechanistic kinetic model is developed along with photoreactor design and modeling for a laboratory-scale batch recirculating photoreactor. 
Continuous-distribution kinetics using population balance equations is applied to describe the dynamics of polymer degradation. In order to validate the proposed model, the model predictions are compared to the experimental data carried out at different operating conditions. Furthermore, in order to determine spatial distribution of different parameters, the computational fluid dynamics (CFD) modeling incorporating rigorous kinetic rate expressions and governing transport equations is also studied.

Finally, a methodology for scaling-up a laboratory-scale photoreactor for the polymer photodegradation is proposed. In this procedure, the intrinsic kinetic parameters are determined using the laboratory-scale batch recirculating photoreactor. The obtained kinetic parameters are used to predict the performance of the pilot-scale continuous flow through photoreactor without any adjustable parameter. Three compulsory conditions are needed in this approach: (1) to hae a validated kinetic scheme, (2) a validated intrinsic reaction kinetic expression using a laboratoryscale photoreactor with the simplest mathematical representation for both mass and radiation balances, and (3) a rigorous mathematical model for the pilot-scale photoreactors. A CFD model development for the scaling-up methodology is validated experimentally in the pilot-scale photoreactor. 


\section{Acknowledgment}

I am highly indebted to several people for their extended help given to me during the work of this entire thesis. This would not have been possible without them. It is a pleasure to thank those who made this possible and I would like to express my sincere gratitude and appreciation to my supervisor, Professor Mehrab Mehrvar, for providing me the unique opportunity to work on this research area and his expert guidance and mentorship, and his encouragement and support at all levels. I also acknowledge Professor Philip Chan as my co-supervisor for his insight and perspective into my work. To each of them, I owe a great debt of gratitude for their patience and inspiration. My sincere thanks is also extended to Professor Hugo De Lasa (the University of Western Ontario, London, Ontario, Canada), the external member of my Ph.D. thesis committee. My regards are also due to Professors D. Foucher (Department of Chemistry and Biology), R. Dhib, and S. Upreti (Department of Chemical Engineering), the committee members of my Ph.D. thesis.

I would like to thank Mr. Ali Hemmati, Mr. Daniel Boothe, and Mr. Tondar Tajrobekar for their help with experimental setups and analytical instruments. I am also indebted to my fellow students: Laila Pakzad, Parisa Tahvildarian, Dr. Masroor Mohajerani, Mauricio Barrera, Amir Mowla, and Ciro LeCompte. A special thank goes to Noel Jacob for helping me in computer programming. The financial support from Natural Sciences and Engineering Research Council of Canada (NSERC) and Ryerson University is greatly acknowledged.

Also, last but not least, I would like to express my honest and eternal gratitude towards my wonderful husband, Ali for his unconditional care and support. He has shown everlasting love, tireless patience and encouragement. Also, I would like to thank my parents, my family, and inlaws for their help, support, and encouragement. 
VITA

Sep. 1998- Feb. 2003.....................Sc. Chemical Engineering, University of Tehran, Iran Sep. 2005-Aug. 2007...........Sc. Chemical Engineering, Sharif University of Technology, Iran Sep. 2008-May 2013 ..................Ph.D. Chemical Engineering, Ryerson University, Canada

The following publications were resulted from this Ph.D. work:

\section{Refereed Journal Papers:}

1. Ghafoori, S., Mehrvar, M., Chan, P. Photoreactor scale-up for degradation of poly(vinyl alcohol) in aqueous solution using $\mathrm{UV} / \mathrm{H}_{2} \mathrm{O}_{2}$ process. Chemical Engineering Journal (submitted).

2. Ghafoori, S., Mehrvar, M., Chan, P. Photoassisted Fenton-like degradation of aqueous poly(acrylic acid): From mechanistic kinetic model to CFD modeling. Chemical Engineering Research and Design (in press).

3. Ghafoori, S., Mehrvar, M., Chan, P. Optimization of photo-Fenton-like degradation of aqueous polyacrylic acid using Box-Behnken experimental design. Canadian Journal of Chemical Engineering, (in press), Published online in May 2013.

4. Ghafoori, S., Mehrvar, M., Chan, P., 2012. Free-radical-induced degradation of aqueous polyethylene oxide by $\mathrm{UV} / \mathrm{H}_{2} \mathrm{O}_{2}$ : Experimental design, reaction mechanisms, and kinetic modeling. Industrial \& Engineering Chemistry Research. 51 (46), 14980-14993, 2012.

5. Ghafoori, S., Mehrvar, M., Chan, P. Kinetic study of photodegradation of water soluble polymers. Iranian Polymer Journal. 21 (12), 869-876, 2012. 
6. Ghafoori, S., Shah, K., Mehrvar, M., Chan, P. Experimental analysis and modeling of pharmaceutical wastewater treatment using combined granular activated carbon and $\mathrm{UV} / \mathrm{H}_{2} \mathrm{O}_{2}$ processes. Canadian Journal of Chemical Engineeing (submitted).

\section{Conference Papers and Presentations:}

1. Ghafoori, S., Mehrvar, M., Chan, P. Determination of intrinsic kinetic constants for scalingup a photoreactor for degradation of poly(vinyl alcohol) using $\mathrm{UV} / \mathrm{H}_{2} \mathrm{O}_{2}$ process. $7^{\text {th }} I W A$ Specialized Membrane Technology Conference and Exhibition for Water and Wastewater Treatment and Reuse. Toronto, August 25-29, 2013.

2. Ghafoori, S., Mehrvar, M., Chan, P. Scaling-up a photoreactor for degradation of poly(ethylene oxide) in aqueous solution. $5^{\text {th }}$ Canadian Wastewater Management Conference $\& 48^{\text {th }}$ Central Canadian Symposium on Water Quality Research. Hamilton, Ontario, March 6-8, 2013.

3. Ghafoori, S., Mehrvar, M., Chan, P. Process optimization of photo-Fenton-like degradation of poly(acrylic acid). International Conference on Chemical Engineering (ICCE'12). San Francisco, USA, October 24-26, 2012.

4. Ghafoori, S., Mehrvar, M., Chan, P. Kinetic study and CFD modeling of poly(acrylic acid) degradation by photo-Fenton-like process. $62^{\text {nd }}$ Canadian Chemical Engineering Conference, Vancouver, British Columbia, October 14-17, 2012.

5. Ghafoori, S., Mehrvar, M., Chan, P. A statistical experiment design approach for the photoFenton-like degradation of polyacrylic acid. $62^{\text {nd }}$ Canadian Chemical Engineering Conference, Vancouver, British Columbia, October 14-17, 2012. 
6. Ghafoori, S., Mehrvar, M., Chan, P. Optimization of aqueous polyethylene oxide photodegradation using design of experiment. 47th Central Canadian Symposium on Water Quality Research. Canada Centre for Inland Waters, Burlington, Ontario. February 21-22, 2012.

7. Ghafoori, S., Mehrvar, M., Chan, P. Photoinduced degradation of poly (N-vinylpyrrolidone) in aqueous solution by iron salt: Degradation mechanism and kinetic modeling. 61st Canadian Chemical Engineering Conference. London, Ontario. Oct 24-26, 2011.

8. Ghafoori, S., Mehrvar, M., Chan, P. Photodegradation of poly (ethylene oxide) by $\mathrm{UV} / \mathrm{H}_{2} \mathrm{O}_{2}$ process: Experimental design and kinetic modeling approach. 61st Canadian Chemical Engineering Conference. London, Ontario. Oct 24-26, 2011.

9. Ghafoori, S., Mehrvar, M., Chan, P. TOC Removal of pharmaceutical wastewater using combined granular activated carbon and $\mathrm{UV} / \mathrm{H}_{2} \mathrm{O}_{2}$ processes. 61st Canadian Chemical Engineering Conference. London, Ontario. Oct 24-26, 2011.

10. Ghafoori, S., Mehrvar, M., Chan, P. Photodegradation kinetics of poly (ethylene) glycol in aqueous solution. 46th Central Canadian Symposium on Water Quality Research. Burlington, Ontario. February. 21-22, 2011.

11. Ghafoori, S., Mehrvar, M., Chan, P. Mechanism and kinetic modeling of photodegradation of polymer-based composites in aqueous solution. The 2nd International Conference on Composites: Characterization, Fabrication and Application. Kish Island, Iran. Dec. 27-30, 2010. 


\section{Dedication}

This thesis is dedicated to my beloved husband, Ali. 


\section{List of Figures}

Figure 2.1. Idealized scheme of photo-initiated oxidation reactions based on the presence of auxiliary oxidants (Adapted by Oppenländer, 2003).

Figure 2.2. Radical chain mechanism of the photo-induced decomposition of $\mathrm{H}_{2} \mathrm{O}_{2}$ (Adapted by

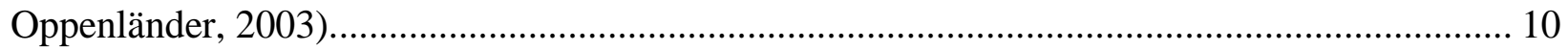
Figure 2.3. Generally accepted mechanism of oxidation by $\mathrm{UV} / \mathrm{H}_{2} \mathrm{O}_{2}$ process (Oppenländer,

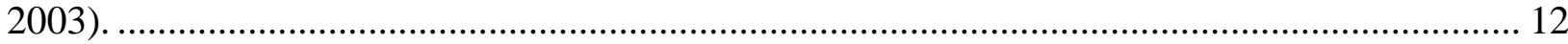

Figure 2.4. Flowchart of evaluation of local volumetric rate of energy absorption (LVREA)... 18

Figure 2.5. General mechanism of photodegradation of polymers........................................ 27

Figure 2.6. Stoichiometric coefficient (kernel) for several values of $m$. (Adapted by McCoy and

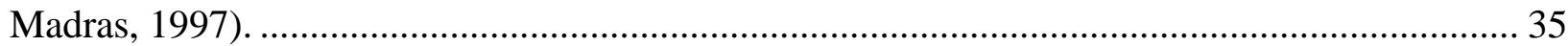

Figure 2.7. Comparison of the concentration-time profiles in a batch (a) and a batch recirculation photoreactor (PR) system (b) (Oppenländer, 2003) ....................................................... 40

Figure 3.1. Schematic diagram of the laboratory-scale batch recirculating system.................. 52

Figure 3.2. Schematic diagram of the pilot-scale flow through system.................................. 54

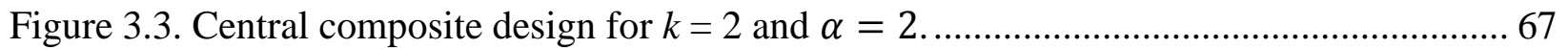

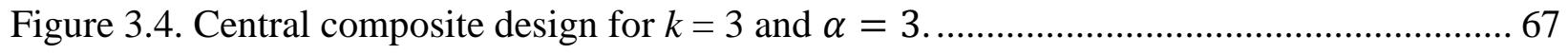

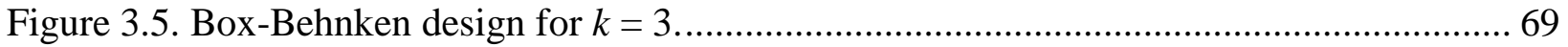

Figure 4.1. Comparison of the kinetic model predictions with the experimental data for number average molecular weight $\left(M_{n}\right)$ and number of chain scission per molecule $(S)$...................... 84

Figure 4.2. Comparison of predicted number average molecular weight versus actual values.... 85 Figure 4.3. Comparison of predicted number average molecular weight versus actual values.... 85 Figure 4.4. Simulation results for the concentration of hydrogen peroxide and total molar concentration of the polymer (zero moment) ................................................................ 86

Figure 4.5. TOC removal of PEO solution by $\mathrm{UV} / \mathrm{H}_{2} \mathrm{O}_{2}$ process $\left([\mathrm{PEO}]_{\mathrm{o}}=10 \mathrm{mg} \mathrm{L}^{-1}\right) \ldots \ldots \ldots \ldots . . . . . . .92$

Figure 4.6. TOC removal of PEO solution by $\mathrm{UV} / \mathrm{H}_{2} \mathrm{O}_{2}$ process $\left([\mathrm{PEO}]_{\mathrm{o}}=20 \mathrm{mg} \mathrm{L}^{-1}\right) \ldots \ldots \ldots \ldots . . . . .92$

Figure 4.7. TOC removal of PEO solution by $\mathrm{UV} / \mathrm{H}_{2} \mathrm{O}_{2}$ process $\left([\mathrm{PEO}]_{\mathrm{o}}=30 \mathrm{mg} \mathrm{\textrm {L } ^ { - 1 }}\right) \ldots \ldots \ldots \ldots . . . . . .93$

Figure 4.8. TOC removal of PEO solution by $\mathrm{UV} / \mathrm{H}_{2} \mathrm{O}_{2}$ process $\left([\mathrm{PEO}]_{\mathrm{o}}=40 \mathrm{mg} \mathrm{L}^{-1}\right) \ldots \ldots \ldots \ldots . . . . . . .93$

Figure 4.9. TOC removal of PEO solution by $\mathrm{UV} / \mathrm{H}_{2} \mathrm{O}_{2}$ process $\left([\mathrm{PEO}]_{\mathrm{o}}=50 \mathrm{mg} \mathrm{L}^{-1}\right) \ldots \ldots \ldots \ldots . . . . . .94$

Figure 4.10. Normal probability plot of the internally studentized residuals......................... 100

Figure 4.11. Comparison of predicted percent TOC removal versus actual values. ................. 100

Figure 4.12. The effects of PEO concentration $\left(X_{1}\right)$ and $\mathrm{H}_{2} \mathrm{O}_{2}$ dosage $\left(X_{2}\right)$ on percent TOC

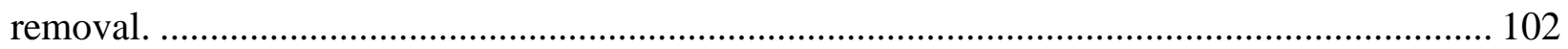

Figure 4.13. The effects of PEO concentration $\left(X_{1}\right)$ and $\mathrm{pH}\left(X_{3}\right)$ on Percent TOC removal..... 102 Figure 4.14. The effects of PEO concentration $\left(X_{1}\right)$ and recirculation rate $\left(X_{4}\right)$ on percent TOC

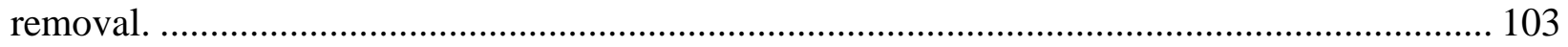
Figure 4.15. The effects of $\mathrm{H}_{2} \mathrm{O}_{2}$ concentration $\left(X_{2}\right)$ and $\mathrm{pH}\left(X_{3}\right)$ on percent TOC removal.... 103 
Figure 4.16. The effects of $\mathrm{H}_{2} \mathrm{O}_{2}$ concentration $\left(X_{2}\right)$ and recirculation rate $\left(X_{4}\right)$ on percent TOC

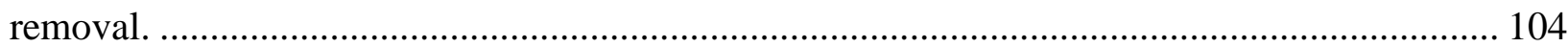

Figure 4.17. The effects of $\mathrm{pH}\left(X_{3}\right)$ and recirculation rate $\left(X_{4}\right)$ on percent TOC removal. ........ 104 Figure 4.18. Comparison of experimental data (•:10 $\mathrm{mg} \mathrm{L}^{-1} \mathrm{PEO} ; \mathbf{m :} 20 \mathrm{mg} \mathrm{L}^{-1} \mathrm{PEO} ; \boldsymbol{\Delta}: 30 \mathrm{mg}$ $\mathrm{L}^{-1} \mathrm{PEO}$; $50 \mathrm{mg} \mathrm{L}^{-1} \mathrm{PEO}$ ) and corresponding model predictions (lines) of $\mathrm{TOC}^{\mathrm{TOC}} \mathrm{C}_{\mathrm{o}}$ as a function of time for $\left[\mathrm{H}_{2} \mathrm{O}_{2}\right]_{0}=300 \mathrm{mg} \mathrm{L}^{-1}$ 114 Figure 4.19. Comparison of experimental data $\left(\bullet: 10 \mathrm{mg} \mathrm{L}^{-1} \mathrm{PEO} ; \mathbf{m}: 20 \mathrm{mg} \mathrm{L}^{-1} \mathrm{PEO} ; \mathbf{\Delta}: 30 \mathrm{mg}\right.$ $\mathrm{L}^{-1} \mathrm{PEO}$; $50 \mathrm{mg} \mathrm{L}^{-1} \mathrm{PEO}$ ) and corresponding model predictions (lines) of $\mathrm{TOC}^{\mathrm{TOC}} \mathrm{o}_{\mathrm{o}}$ as a

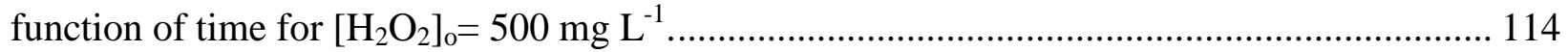

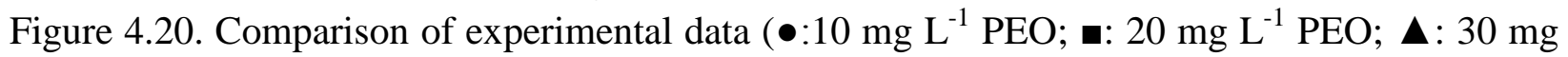
$\mathrm{L}^{-1} \mathrm{PEO}$; $50 \mathrm{mg} \mathrm{L}^{-1} \mathrm{PEO}$ ) and corresponding model predictions (lines) of $\mathrm{TOC}^{\mathrm{TOC}} \mathrm{o}_{\mathrm{o}}$ as a

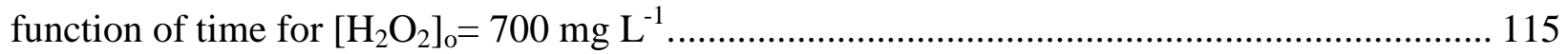
Figure 4.21. Comparison of experimental data (•:10 $\mathrm{mg} \mathrm{L}^{-1} \mathrm{PEO} ; \mathbf{m}: 20 \mathrm{mg} \mathrm{L}^{-1} \mathrm{PEO} ; \boldsymbol{\Delta}: 30 \mathrm{mg}$ $\mathrm{L}^{-1} \mathrm{PEO}$; $50 \mathrm{mg} \mathrm{L}^{-1} \mathrm{PEO}$ ) and corresponding model predictions (lines) of $\mathrm{TOC}^{\mathrm{TOOC}} \mathrm{o}_{\mathrm{o}}$ as a function of time for $\left[\mathrm{H}_{2} \mathrm{O}_{2}\right]_{\mathrm{o}}=900 \mathrm{mg} \mathrm{L}^{-1}$...... 115 Figure 4.22. Comparison of experimental data $\left(\bullet: 10 \mathrm{mg} \mathrm{L}^{-1} \mathrm{PEO} ; \mathbf{m}: 20 \mathrm{mg} \mathrm{L}^{-1} \mathrm{PEO} ; \boldsymbol{\Delta}: 30 \mathrm{mg}\right.$ $\mathrm{L}^{-1} \mathrm{PEO}$; $: 50 \mathrm{mg} \mathrm{L}^{-1} \mathrm{PEO}$ ) and corresponding model predictions (lines) of $\mathrm{TOC}^{\mathrm{TOC}} \mathrm{o}_{\mathrm{o}}$ as a

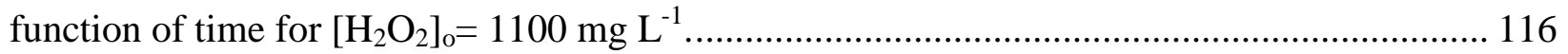
Figure 4.23. Percent TOC removal vs. initial $\mathrm{H}_{2} \mathrm{O}_{2}$ dosage for different PEO concentration.... 118 Figure 4.24. Algorithm of mathematical modeling and computational simulation procedure for kinetic parameter estimation..................................................................................... 119 Figure 4.25. Normal probability plot of the residuals.................................................... 131

Figure 4.26. Comparison of predicted TOC removal rates versus actual values...................... 131 Figure 4.27. Interaction effect between $[\mathrm{PAA}]_{\mathrm{o}}$ and $\left[\mathrm{Fe}^{3+}\right]_{\mathrm{o}}$ on percent TOC removal.............. 135 Figure 4.28. Interaction effect between $[\mathrm{PAA}]_{\mathrm{o}}$ and $\left[\mathrm{H}_{2} \mathrm{O}_{2}\right]_{\mathrm{o}}$ on percent TOC removal............ 135 Figure 4.29. Interaction effect between $\left[\mathrm{Fe}^{3+}\right]_{\mathrm{o}}$ and $\left[\mathrm{H}_{2} \mathrm{O}_{2}\right]_{\mathrm{o}}$ on percent TOC removal............. 136 Figure 4.30. Simulation of the proposed kinetic model and comparison with experimental data shown in Table 4.11: Exp.1 (口), Exp.2 ( $\nabla)$, and Exp.3 (*)........................................... 145 Figure 4.31. Simulation of the proposed kinetic model and comparison with experimental data shown in Table 4.11: Exp.4 (+), Exp.5 (ぬ), Exp.6 (o)..................................................... 145

Figure 4.32. Simulation of the proposed kinetic model and comparison with experimental data shown in Table 4.11: Exp.7 ( $\square)$, Exp.8 (+), Exp.9 $(\nabla)$.................................................... 146 Figure 4.33. Comparison of model predictions with experimental data................................. 146 Figure 4.34. The peak values of the normalized sensitivity coefficients for TOC concentration with respect to reaction rate constant for the most important chemical reactions in Table 4.10.149 Figure 4.35. Laboratory-scale photoreactor geometry (dimensions are given in centimeters). . 151 Figure 4.36. CFD simulation for axial velocity in laboratory-scale photoreactor (a) top view (b) side view. 155 Figure 4.37. CFD simulation for TOC concentration profile in laboratory-scale photoreactor (a) side view (b) top view. 156 
Figure 4.38. CFD simulation for $\mathrm{Fe}^{3+}$ concentration profile in laboratory-scale photoreactor. . 158 Figure 4.39. CFD simulation for $\mathrm{Fe}^{2+}$ concentration profile in laboratory-scale photoreactor. . 158 Figure 4.40. CFD simulation for ${ }^{\circ} \mathrm{OH}$ concentration profile in laboratory-scale photoreactor... 159 Figure 4.41. CFD simulation for LVREA in laboratory-scale photoreactor (a) top view (b) cross section. 160

Figure 4.42. TOC removal (norm) in one photoreactor for $\mathrm{UV}, \mathrm{UV} / \mathrm{H}_{2} \mathrm{O}_{2}, \mathrm{UV} / \mathrm{Fe}^{3+}$, and $\mathrm{UV} / \mathrm{H}_{2} \mathrm{O}_{2} / \mathrm{Fe}^{3+}$ processes. 166

Figure 4.43. TOC removal (norm) in two photoreactors in series for $\mathrm{UV}, \mathrm{UV} / \mathrm{H}_{2} \mathrm{O}_{2}, \mathrm{UV} / \mathrm{Fe}^{3+}$, and $\mathrm{UV} / \mathrm{H}_{2} \mathrm{O}_{2} / \mathrm{Fe}^{3+}$ processes. . 166

Figure 4.44. "Pseudo"-second order kinetic fit for experimentally achieved TOC in 27 runs... 172 Figure 4.45. Observed vs. predicted plot (a), normal probability plot (b), and internally studentized residuals vs. predicted values plot (c) for the prediction of percent TOC removal in photo-Fenton-like process using two photoreactors in series. 178 Figure 4.46. Observed vs. predicted plot (a), normal probability plot (b), and internally studentized residuals vs. predicted values plot (c) for the prediction of "pseudo"-second order rate constant in photo-Fenton-like process using two photoreactors in series. 179 Figure 4.47. 3D response surface and $2 \mathrm{D}$ contours showing the interaction effects of the factors on the percent TOC removal: (a) $[\mathrm{PAA}]_{\mathrm{o}}\left(X_{1}\right)$ and $\left[\mathrm{H}_{2} \mathrm{O}_{2}: \mathrm{Fe}^{3+}\right]_{\mathrm{o}}\left(X_{2}\right)$, (b) $[\mathrm{PAA}]_{\mathrm{o}}$ and $\mathrm{pH}\left(X_{3}\right)$, (c) $[\mathrm{PAA}]_{\mathrm{o}}$ and recirculation rate $\left(X_{4}\right)$, (d) $\left[\mathrm{H}_{2} \mathrm{O}_{2}: \mathrm{Fe}^{3+}\right]_{\mathrm{o}}$ and $\mathrm{pH}$, (e) $\left[\mathrm{H}_{2} \mathrm{O}_{2}: \mathrm{Fe}^{3+}\right]_{\mathrm{o}}$, (f) $\mathrm{pH}$ and recirculation rate.

Figure 4.48. 3D response surface and $2 \mathrm{D}$ contours showing the interaction effects of the factors "pseudo"-second order rate constant: (a) $[\mathrm{PAA}]_{\mathrm{o}}\left(X_{1}\right)$ and $\left[\mathrm{H}_{2} \mathrm{O}_{2}: \mathrm{Fe}^{3+}\right]_{\mathrm{o}}\left(X_{2}\right)$, (b) $[\mathrm{PAA}]_{\mathrm{o}}$ and $\mathrm{pH}$ $\left(X_{3}\right)$, (c) $[\mathrm{PAA}]_{\mathrm{o}}$ and recirculation rate $\left(X_{4}\right)$, (d) $\left[\mathrm{H}_{2} \mathrm{O}_{2}: \mathrm{Fe}^{3+}\right]_{\mathrm{o}}$ and $\mathrm{pH}$, (e) $\left[\mathrm{H}_{2} \mathrm{O}_{2}: \mathrm{Fe}^{3+}\right]_{\mathrm{o}}$ and recirculation rate, (f) $\mathrm{pH}$ and recirculation rate. 181

Figure 4.49. Photoreactor Scale-up methodology..... 188 Figure 4.50. Experimental and predicted $\mathrm{TOC} \mathrm{TOC}_{\mathrm{o}}$ concentration vs. time for PVA=20 mg L $\mathrm{m}^{-1}$ in laboratory-scale photoreactor. Lines are model predictions and the symbols are corresponding experimental results. 194 Figure 4.51. Kinetic model predictions vs. experimental results for TOC concentration of photodegradation of PVA by $\mathrm{UV} / \mathrm{H}_{2} \mathrm{O}_{2}$ at different dosage of $\mathrm{H}_{2} \mathrm{O}_{2}$ in laboratory-scale photoreactor $\left(300,500,700,900\right.$, and $\left.1100 \mathrm{mg} \mathrm{L}^{-1}\right)$..................................................... 195

Figure 4.52. Pilot-scale photoreactor geometry (dimension are given in centimeters). ............ 197

Figure 4.53. Schematic diagram of a three-dimensional source with volumetric emission model (Adapted by Cassano et al., 1995)..................................................................................... 199

Figure 4.54. Continuous flow annular photoreactor (Adapted by Cassano et al., 1995)........... 203

Figure 4.55. Limits of integration for the lamp (Adapted by Cassano et al., 1995)................. 204

Figure 4.56. Local volumetric rate of energy absorption in the pilot-scale photoreactor.......... 206 Figure 4.57. Velocity profile in (a) axial and (b) radial cross section of the pilot-scale photoreactor (PVA: $20 \mathrm{mg} \mathrm{L}^{-1}, \mathrm{H}_{2} \mathrm{O}_{2}$ : $900 \mathrm{mg} \mathrm{L}^{-1}$, and flow rate: $0.1 \mathrm{~L} \mathrm{~min}^{-1}$ ). 209 
Figure 4.58. TOC concentration profile for PVA in the pilot-scale photoreactor (PVA: $20 \mathrm{mg} \mathrm{L}^{-1}$,

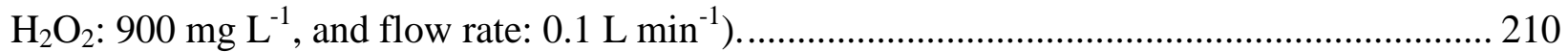

Figure 4.59. Convective flux for PVA in the pilot-scale photoreactor (PVA: $20 \mathrm{mg} \mathrm{L}^{-1}, \mathrm{H}_{2} \mathrm{O}_{2}$ :

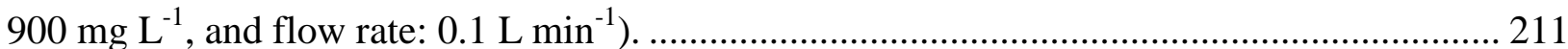

Figure 4.60. Diffusive flux for PVA in the pilot-scale photoreactor (PVA: $20 \mathrm{mg} \mathrm{L}^{-1}, \mathrm{H}_{2} \mathrm{O}_{2}: 900$

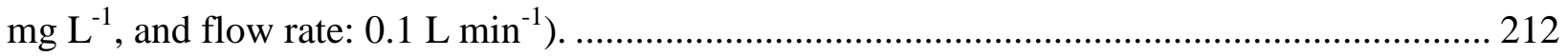
Figure 4.61. Total flux for PVA in the pilot-scale photoreactor (PVA: $20 \mathrm{mg} \mathrm{L}^{-1}, \mathrm{H}_{2} \mathrm{O}_{2}: 900 \mathrm{mg}$ $\mathrm{L}^{-1}$, and flow rate: $0.1 \mathrm{~L} \mathrm{~min}^{-1}$ ). 213 Figure 4.62. Concentration profile for $\mathrm{H}_{2} \mathrm{O}_{2}$ in the pilot-scale photoreactor (PVA: $20 \mathrm{mg} \mathrm{L}^{-1}$, $\mathrm{H}_{2} \mathrm{O}_{2}: 900 \mathrm{mg} \mathrm{L}^{-1}$, and flow rate: $0.1 \mathrm{~L} \mathrm{~min}^{-1}$ ).

Figure 4.63. Convective flux for $\mathrm{H}_{2} \mathrm{O}_{2}$ in the pilot-scale photoreactor (PVA: $20 \mathrm{mg} \mathrm{L}^{-1}, \mathrm{H}_{2} \mathrm{O}_{2}$ : $900 \mathrm{mg} \mathrm{L}^{-1}$, and flow rate: $0.1 \mathrm{~L} \mathrm{~min}^{-1}$ ).

Figure 4.64. Diffusive flux for $\mathrm{H}_{2} \mathrm{O}_{2}$ in the pilot-scale photoreactor (PVA: $20 \mathrm{mg} \mathrm{L}^{-1}, \mathrm{H}_{2} \mathrm{O}_{2}: 900$ $\mathrm{mg} \mathrm{L}{ }^{-1}$, and flow rate: $0.1 \mathrm{~L} \mathrm{~min}^{-1}$ ). 216

Figure 4.65. Total flux for $\mathrm{H}_{2} \mathrm{O}_{2}$ in the pilot-scale photoreactor (PVA: $20 \mathrm{mg} \mathrm{L}^{-1}, \mathrm{H}_{2} \mathrm{O}_{2}: 900 \mathrm{mg}$ $\mathrm{L}^{-1}$, and flow rate: $0.1 \mathrm{~L} \mathrm{~min}^{-1}$ ). 217 Figure 4.66. CFD Model prediction vs. experimental data for percent TOC removal in the pilotscale photoreactor at different $\mathrm{H}_{2} \mathrm{O}_{2}$ dosage $\left(300,500,700,900,1100 \mathrm{mg} \mathrm{L}^{-1}\right)$ and different inlet flow rate $\left(\boldsymbol{\Delta}: 0.1 \mathrm{~L} \mathrm{~min}^{-1}, \bullet: 0.3 \mathrm{~L} \mathrm{~min}^{-1}\right.$, and $\left.\bullet: 0.5 \mathrm{~L} \mathrm{~min}^{-1}\right)$. 218

Figure A.1. TOC calibration curve plotted for output raw data from the detector versus the amount of organic carbon present for the range of 1-4000 $\mathrm{m} \mathrm{C} \mathrm{L} \mathrm{L}^{-1}$ .248

Figure A.2. TOC calibration curve for the range 0-60 $\mu \mathrm{gC}$ from Apollo $9000 \mathrm{TOC}$ analyzer...249 


\section{List of Tables}

Table 2.1. Radiation type and associated energy ........................................................... 5

Table 2.2. Synthetic water-soluble polymers of potential environmental concern..................... 45

Table 3.1. Description of laboratory-scale photoreactor. ................................................... 53

Table 3.2. Description of pilot-scale photoreactor............................................................. 55

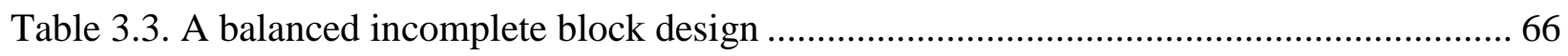

Table 4.1. Reaction mechanisms of the photodegradation in $\mathrm{UV} / \mathrm{H}_{2} \mathrm{O}_{2}$ process........................ 75

Table 4.2. Independent variables and their coded levels for $\mathrm{UV} / \mathrm{H}_{2} \mathrm{O}_{2}$ process. ....................... 95

Table 4.3. Four-factor Five-level CCD for RSM and the observed and predicted responses. ..... 97

Table 4.4. ANOVA for prediction of percent TOC removal by the quadratic model (CCD)..... 98

Table 4.5. Reaction mechanism for complete mineralization of $\mathrm{PEO}$ by $\mathrm{UV} / \mathrm{H}_{2} \mathrm{O}_{2} \ldots \ldots \ldots \ldots \ldots . . . . .105$

Table 4.6. Optimization and simulation results for the kinetic rate constants......................... 123

Table 4.7. Independent variables and their coded levels based on Box-Behnken design for photo-

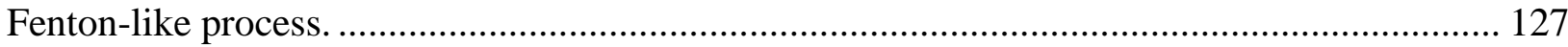

Table 4.8. Three-factor three-level Box-Behnken design for RSM...................................... 129

Table 4.9. ANOVA for prediction of TOC removal rate by the quadratic model..................... 130

Table 4.10. Reaction mechanism for complete mineralization of PAA by UV/Fe ${ }^{3+} / \mathrm{H}_{2} \mathrm{O}_{2} \ldots \ldots .137$

Table 4.11. Experimental conditions of $\mathrm{UV} / \mathrm{H}_{2} \mathrm{O}_{2} / \mathrm{Fe}^{3+}$ process in 9 different sets.................. 143

Table 4.12. Laboratory-scale photoreactor description in continuous flow. ............................ 152

Table 4.13. Independent variables and their coded levels based on Box-Behnken design for

photo-Fenton-like process using two photoreactors in series. ............................................ 168

Table 4.14. Four-factor Box-Behnken design and the observed and predicted responses. ........ 173

Table 4.15. ANOVA results for prediction of percent TOC removal in photo-Fenton-like process

using two photoreactors in series.................................................................................... 174

Table 4.16. ANOVA results for prediction of "pseudo"-second order rate constant in photoFenton-like process using two photoreactors in series. ...................................................... 175

Table 4.17. Pilot-scale photoreactor description in continuous flow...................................... 198 


\section{Chapter 1}

\section{INTRODUCTION}

Water-soluble polymers are produced in large volumes and due to their solubility in water, they are widely used in a variety of industrial products and applications including pharmaceuticals, paints, adhesives, coatings, textiles, and detergents (Bénard et al., 2011). These polymers can be natural or synthetic with an array of molecular chemistry, structures, and sizes. The synthetic water-soluble polymers are gaining more attention due to the industrial development. Some of the key functionalities of these substances are their ability to increase the viscosity of solutions, form physical gels, stabilize dispersions and emulsions, induce particle aggregation, and solubilise hydrophobic compounds. Some examples of synthetic water-soluble polymers are poly (vinyl alcohol), poly (acryl amide), poly (acrylic acid), poly (vinyl pyrrolidine), poly (ethylene oxide), and polyisocyanates.

After industrial use, most of the water-soluble polymers are discarded into aqueous environments. Therefore, they can reach conventional sewage disposal systems and subsequently contaminate water resources. The contribution of this type of polymers to the ever-increasing problem of pollution is a major concern due to their water solubility that makes their environmental fate less obvious to the general public. Consequently, these polymers have received less attention in public than packaging plastics, which are visibly discarded in environment. On the other hand, they are not readily biodegradable by the type of microorganisms which normally attack most other forms of organic matter, thus rendering them to the biological life cycle (Reich and Stivala, 1971). Also, most of the techniques currently applied to degrade these types of polymers have been found either inefficient or hazardous to the 
environment. Hence, the possibility of degrading these materials to harmless and biologically useful chemical compounds is a challenging task.

In recent decades, advanced oxidation technologies (AOTs) have been applied successfully to degrade recalcitrant pollutants in wastewater (Mohajerani et al., 2009; Poyatos et al., 2010; Barrera et al., 2012). AOTs involve the use of UV radiation, $\mathrm{O}_{3}, \mathrm{H}_{2} \mathrm{O}_{2}$, photocatalysts (such as $\mathrm{TiO}_{2}$ ), metal catalysts (such as $\mathrm{Fe}$ in Fenton's reaction) or any combination thereof to generate short-lived, non-selective chemical species of high oxidation potential. The free radical species (especially hydroxyl radicals) mediate fast degradation or decomposition of target organic compounds in wastewater. The unique feature of AOTs in wastewater treatment is that in most of processes, they do not generate sludge waste or volatile organic compounds associated with most conventional treatment processes. One of the main drawbacks of AOTs is their high operating cost in comparison to other conventional methods. To overcome this problem, AOTs are coupled with other treatment methods. They are often employed as a pre-treatment method to biodegradation in an integrated system. On the other hand, AOTs are multifactor systems that various parameters such as concentration of organic materials, light intensity, oxidant dosage, reaction time, $\mathrm{pH}$, and other operating conditions influence the efficiency of the system. Therefore, in addition to the single-factor effects, cross-factor effects must be taken into consideration in order to characterize such systems.

However, only few studies are available in the open literature on the degradation and mineralization of polymers by different methods of AOTs (Konaganti et al., 2009; Hassouna et al., 2011). Also, there is a lack of information on the mechanism and kinetic modeling of AOTs for synthetic water-soluble polymers because of the complexity of the reactions involving molecules with high molecular weights. Therefore, in this study, the effectiveness of the 
degradation of synthetic water-soluble polymer in wastewater by different methods of AOTs ( $\mathrm{UV} / \mathrm{H}_{2} \mathrm{O}_{2}$ and $\mathrm{UV} / \mathrm{Fe}^{3+} / \mathrm{H}_{2} \mathrm{O}_{2}$ ) using experimental design, statistical analysis, and optimization along with the photoreactor design, photoreactor modeling, scaling-up methodology, and CFD model development will be investigated.

Chapter 2 presents the background and a comprehensive literature review on the AOTs, general mechanism of AOTs, kinetics of photolysis, photochemical degradation of polymers, photoreactor design, photorector modeling, and scaling-up methodology. In Chapter 3 the experimental setups (laboratory-scale and pilot-scale photoreactors), analytical techniques, experimental design using different methods of response surface methodology, and the CFD modeling methodology are explained. Chapter 4 presents the results and discussion for different AOTs including the experimental design, statistical analysis, optimization, kinetic model development, CFD modeling, and scaling-up methodology. In Chapter 5, the conclusions and recommendations are discussed. 


\section{Chapter 2}

\section{LITERATURE BACKGROUND}

\subsection{ADVANCED OXIDATION TECHNOLOGIES (AOTs)}

The applications of advanced oxidation technologies (AOTs) for treating wastewater containing recalcitrant and inhibitory organics have risen drastically during the past few decades (Poyatos et al., 2010; Mohajerani et al., 2010; Wols et al., 2012). Besides from transforming chemical pollutants, AOTs have advantages of complete mineralization of some organic compounds, producing by-products with little hazard to the environment, and oxidation of organic compounds at very low concentration (Oppenländer, 2003; Tabrizi et al., 2004; Edalatmanesh et al., 2008b; Cao and Mehrvar, 2011; Mohajerani et al., 2012a,b;). In fact, AOTs render the contaminants to less toxic intermediary oxidation products that can be accessible to efficient biological treatment (Pulgarin and Kiwi, 1996; Adams and Kuzhikannil, 2000; Ledakowicz and Solecka, 2000; Tabrizi and Mehrvar, 2004). The unique feature of AOTs in wastewater treatment is that they do not generate waste sludge or volatile organic compounds associated with most conventional treatment processes. Another advantage of AOTs is that they can be applied at ambient pressure and room temperature. Therefore, much research has been carried out based on the AOTs for water and wastewater treatment during the past 30 years. One of the main drawbacks of AOTs is their high operating cost in comparison to other conventional methods. To overcome this problem, AOTs are coupled to other treatment methods. They are often employed as a pre-treatment method to the biodegradation in an integrated system. Some fundamental principles of AOTs are presented here. 
UV radiation consists of energy from $300 \mathrm{~kJ}^{E^{2}}$ instein $^{-1}$ (UV-A radiation) up to $1200 \mathrm{~kJ}$ Einstein $^{-1}$ (vacuum UV radiation). The energy and wavelengths of different UV radiation types are classified in Table 2.1.

Table 2.1. Radiation type and associated energy

\begin{tabular}{ccc}
\hline Radiation & Wavelength $(\mathrm{nm})$ & Energy $\left(\mathrm{kJ} \mathrm{Einstein}^{-1}\right)^{\mathrm{a}}$ \\
\hline Visible & $780-400$ & $155-300$ \\
UV-A & $400-315$ & $300-377$ \\
UV-B & $315-280$ & $377-425$ \\
UV-C & $280-100$ & $425-1198$ \\
${ }^{\mathrm{a}} 1$ Einstein $=6.023 \times 10^{23}$ photons $=1$ mole of photons
\end{tabular}

For oxidation and disinfection processes, the UV-C radiation is commonly used with the most common wavelength of $254 \mathrm{~nm}$ due to the development of low pressure mercury lamp by Hewitt in 1901 (Meulemans, 1978).

At room temperature, most of the molecules stay at their lowest energy electronic state which is the "ground state". When the molecule is exposed to any type of radiation with sufficient energy such as UV radiation, the radiation can be absorbed and the molecule will be promoted to an excited state. In this case, one electron of the molecule goes to the higher energy state or excited state. The excited state can be either a singlet in which all electron spins cancel or a triplet that has two unpaired electrons with parallel spins. Generally, the most possible transition takes place from ground state to singlet state. The difference between the energy of the ground state and singlet state, $h v$, represents the absorbed energy in which $h$ is Plank's constant and $v$ is the frequency of the absorbed radiation. The lifetime of the molecule in an excited state is very short $\left(10^{-8}\right.$ to $\left.10^{-9} \mathrm{sec}\right)$ which leads to either retuning to the ground state or decomposing to produce a different short lived highly reactive reagent such as hydroxyl radical $\left({ }^{\circ} \mathrm{OH}\right)$ which can initiate of 
photochemical reactions (Tarr, 2003). The hydroxyl radical has electrophilic properties and its reactions with appropriate substrate molecules are kinetically controlled, usually with very high second order rate constants that are often close to the diffusion-controlled limit. Kinetic reaction control refers to the irreversible reactions in which the product composition is determined by the relative rates of product formation. Therefore, the optimization of AOTs is always required for the efficient production of the so-called "free" hydroxyl radicals at sufficient concentrations from appropriate precursors.

\subsubsection{General Mechanism of AOTs}

Photochemical reactions including chain reactions of oxygen derived species can be classified as follows (Oppenländer, 2003):

- The photo-induced oxidation of molecule $M$ in the presence of oxygen (Reactions (2.1) and (2.2)) in which the molecule promotes to its excited state and subsequently a radical cation (Reaction (2.1)) and photooxidation of excited molecule occurs in the presence of oxygen (Reaction (2.2)).

- Photo-initiated oxidation (Reaction (2.3)) in which $M$ reacts with the short-lived highly reactive radicals which is formed from an electronically excited precursor molecule (more active) $N^{*}$ and this is what AOTs are based on.

- Photooxygenation Reactions (Reactions (2.4) to (2.6)) are complex reactions. Many competitive reaction pathways may lead to various oxygenated products. Type I products are the results of the reaction of free radical formed by the photochemical reactions with ground state molecular oxygen $\left({ }^{3} \mathrm{O}_{2}\right)$ as can be seen in Reaction (2.4). Type II products 
(Reaction (2.5)) are formed by the sensitized photooxidation through singlet molecular oxygen $\left({ }^{1} \mathrm{O}_{2}\right)$. A photosensitizer is an electron or energy transfer agent which is not usually consumed in the photoreaction. The most common sensitizers are organic dyes with great potential of absorbing light. Type III products (Reaction (2.6)) are formed by the reaction of superoxide with a substrate molecule $M$.

$$
\begin{aligned}
& M+h v \rightarrow M^{*} \rightarrow M^{\bullet^{+}}+e^{-} \\
& M+h v \rightarrow M^{*} \stackrel{O_{2}}{\rightarrow} \text { Products } \\
& \left.N+h v \rightarrow N^{*} \rightarrow \text { Reactive Species } \stackrel{M^{, O_{2}}}{\longrightarrow} \text { Products }\right\} \text { Photo-initiated oxidation } \\
& M+h v \rightarrow M^{*} \rightarrow M \cdot \stackrel{\cdot O_{2} \bullet}{\longrightarrow} M-O-O \bullet \rightarrow \text { Products type I }
\end{aligned}
$$

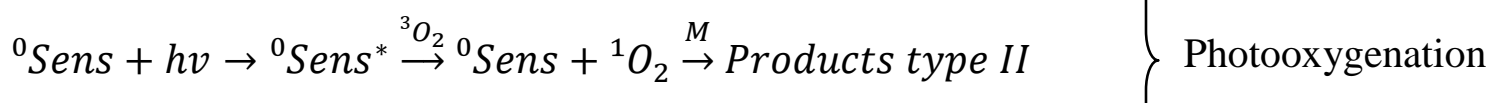

$$
\begin{aligned}
& { }^{0} \text { Sens }+h v \rightarrow \text { Sens }^{*} \stackrel{3^{3} O_{2}}{\rightarrow} \text { Sens }^{{ }^{+}+}+\mathrm{O}_{2}^{\bullet-} \stackrel{M}{\rightarrow} \text { Products type III }
\end{aligned}
$$

where Sens are sensitizers. AOTs are principally based on the photo-initiated Reactions (Reaction (2.3)). However, depending on different circumstances, the photo-induced oxidation or photooxygenation reactions may compete with photo-initiated oxidations. An idealized situation of photo-initiated oxidation reactions that is the basis of all AOTs in the presence of an oxidizer, such as $\mathrm{H}_{2} \mathrm{O}_{2}$ or $\mathrm{O}_{3}$, is depicted in Figure 2.1. By absorbing electromagnetic radiation, the auxiliary oxidant (a-ox) containing oxygen atoms produces an electronically excited molecule (a$\mathrm{ox}^{*}$ ). Consequently, homolytic cleavage of a chemical bond leads to the formation of primary reactive oxygen species $(\mathrm{Ox} \bullet)$ which are mainly hydroxyl radicals, or oxygen atoms in their ground (3P) or excited (1D) states. Since the oxygen-derived species are highly reactive, their 
concentration is usually very low. Immediate reaction with substrate molecules $M$ in the presence of oxygen predominantly initiates a complex series of radical reactions including peroxyl radical chemistry (Sonntag and Schuchmann, 1991; Alfassi, 1997). This reaction scheme is considerably complex as the auxiliary oxidants are able to oxidize certain water contaminants by direct oxidation based on their reduction potential (Simmler, 1995). In the following sections, general mechanism of $\mathrm{UV} / \mathrm{H}_{2} \mathrm{O}_{2}$ and photo-Fenton $\left(\mathrm{UV} / \mathrm{H}_{2} \mathrm{O}_{2} / \mathrm{Fe}^{2+}\right)$, and photo-Fenton-like (UV/H${ }_{2} \mathrm{O}_{2} / \mathrm{Fe}^{3+}$ ) as three methods of AOTs with great potential of oxidation are presented.

\subsubsection{General mechanism of $\mathrm{UV} / \mathrm{H}_{2} \mathrm{O}_{2}$ process}

Although the photochemistry of $\mathrm{H}_{2} \mathrm{O}_{2}$ in solution has been reviewed extensively, it is still of specific interest. Many aspects of $\mathrm{H}_{2} \mathrm{O}_{2}$ chemistry, e.g. the quantum yield of its decomposition, still leads to confusion in the scientific literature. The radical chain mechanism of the photoinduced decomposition of hydrogen peroxide in aqueous solution could be explained by the Haber-Weiss mechanism (Figure 2.2). According to this radical chain mechanism, the homolytic decomposition of $\mathrm{H}_{2} \mathrm{O}_{2}$ in the initiation step leads to the formation of $\mathrm{HO}-\mathrm{OH}$ bond that initiates a propagation cycle with the net reaction leading to water and molecular oxygen. An intermediate six-membered cyclic complex between a hydroperoxyl radical and hydrogen peroxide in the liquid phase could be formed as presented in Figure 2.1. (Einschlag et al., 1997; Oppenländer, 2003). This hydrogen-bonded complex $\left(\mathrm{HOO}^{\circ} . . \mathrm{H}_{2} \mathrm{O}_{2}\right)$ rearranges by oneelectron shifts to yield the final products water, molecular oxygen and a hydroxyl radical. The hydroxyl radical again enters the propagation cycle. The termination step may take place by the recombination of two hydroxyl radicals, the reaction of hydroxyl radical and hydroperoxyl radical, and disproportionation of two hydroperoxyl radicals. 


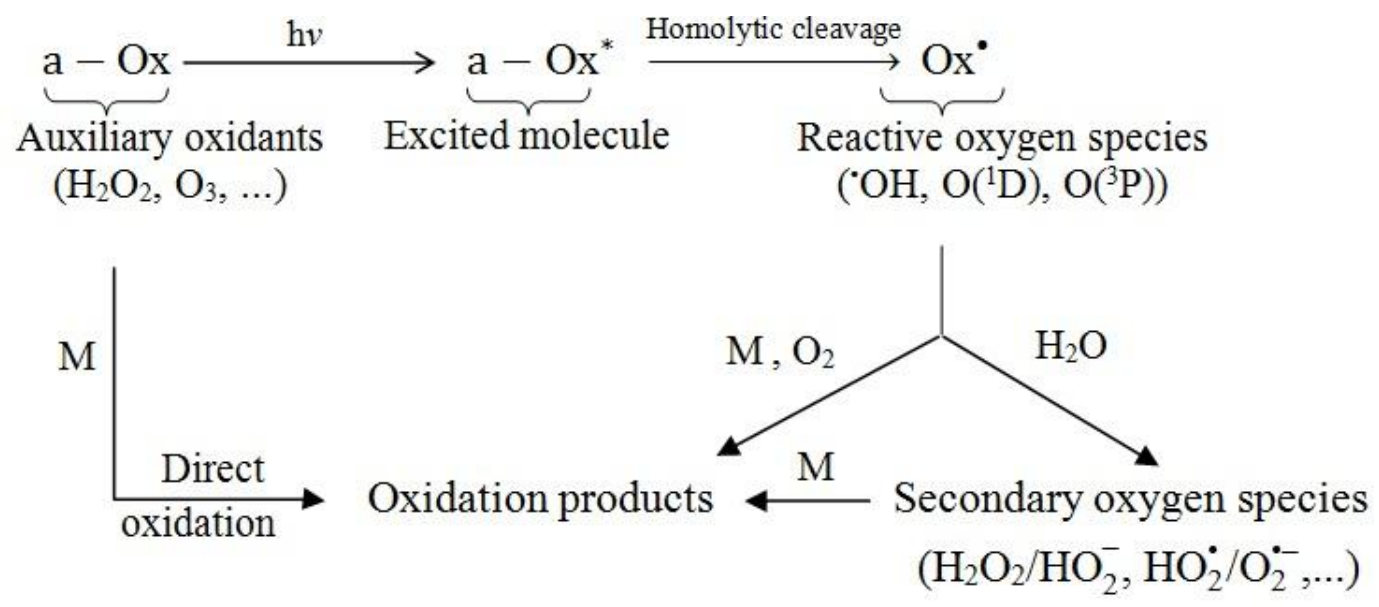

Figure 2.1. Idealized scheme of photo-initiated oxidation reactions based on the presence of auxiliary oxidants (Adapted by Oppenländer, 2003). 


$$
\begin{array}{ll}
\text { Initiation: } & \mathrm{H}_{2} \mathrm{O}_{2} \stackrel{h v}{\rightarrow} 2 \cdot \mathrm{OH} \\
\text { Propagation: } & \mathrm{H}_{2} \mathrm{O}_{2}+\cdot \mathrm{OH} \rightarrow \mathrm{HO}_{2}+\mathrm{H}_{2} \mathrm{O} \\
& \mathrm{HO}_{2}^{\cdot}+\mathrm{H}_{2} \mathrm{O}_{2} \rightarrow \cdot \mathrm{OH}+\mathrm{H}_{2} \mathrm{O}+\mathrm{O}_{2} \\
& 2 \mathrm{H}_{2} \mathrm{O}_{2} \rightarrow 2 \mathrm{H}_{2} \mathrm{O}+\mathrm{O}_{2} \\
\text { Net Reaction: } & \\
& \\
\text { Termination: } & \cdot \mathrm{OH}+\cdot \mathrm{OH} \rightarrow \mathrm{H}_{2} \mathrm{O}_{2} \\
& \cdot \mathrm{OH}+\mathrm{HO}_{2}^{\cdot} \rightarrow \mathrm{H}_{2} \mathrm{O}+\mathrm{O}_{2} \\
& \mathrm{HO}_{2}^{\cdot}+\mathrm{HO}_{2}^{\cdot} \rightarrow \mathrm{H}_{2} \mathrm{O}_{2}+\mathrm{O}_{2}
\end{array}
$$

Figure 2.2. Radical chain mechanism of the photo-induced decomposition of $\mathrm{H}_{2} \mathrm{O}_{2}$ (Adapted by Oppenländer, 2003). 
Therefore, the generated free radicals (mainly hydroxyl radicals) with the greatest potential for oxidation are responsible to attack the organic compounds ( $\mathrm{RH}$ in Figure 2.3) and subsequently leading to the mineralization and oxidation of the target molecules according to Figure 2.3.

In order to understand the chain mechanism better, it should be re-stated that the AOTs are based on two different but interrelated levels: the level of reactive intermediates and the level of oxidation products. The former depends on the specific AOT that is applied (type of the lamp etc) while the latter involves simple oxidation reactions and substrate mineralization. The mineralization of organic matter (i. e. reduction of TOC) in water, with some time delay, usually competes with substrate oxidation (Oppenländer, 2003). However, the efficiency of the TOC reduction is much slower than that of the substrate consumption due to the fact that the reaction of a substrate molecule with one ${ }^{\circ} \mathrm{OH}$ radical already leads to the disappearance of that specific molecule. This primary step is equivalent to the oxidation of the substrate, whereas the mineralization involves a complex series of oxidation and $\mathrm{C}-\mathrm{C}$ bond fragmentation reactions until all $\mathrm{C}$-atoms of the organic molecule are transformed into their highest oxidation state as $\mathrm{CO}_{2}$. By the accumulation of oxidation products and of radical recombination products, the mineralization progress may be hindered significantly.

\subsubsection{General mechanism of photo-Fenton $\left(\mathrm{UV} / \mathrm{H}_{2} \mathrm{O}_{2} / \mathrm{Fe}^{2+}\right)$ and photo- Fenton-like (UV/ $\left./ \mathrm{H}_{2} \mathrm{O}_{2} / \mathrm{Fe}^{3+}\right)$ processes}

The term Fenton reagent refers to aqueous mixtures of $\mathrm{Fe}^{2+}$ and hydrogen peroxide that was first reported by Fenton (Fenton, 1894). Subsequent research after Fenton has elucidated the following net reaction as the principal process: 


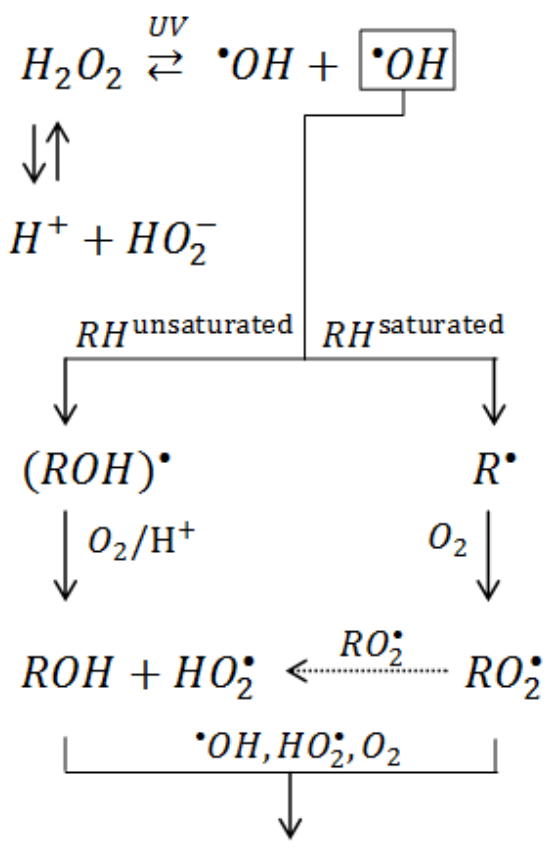

Oxidation and Mineralization

Figure 2.3. Generally accepted mechanism of oxidation by $\mathrm{UV} / \mathrm{H}_{2} \mathrm{O}_{2}$ process (Oppenländer, 2003). 
$\mathrm{Fe}^{2+}+\mathrm{H}_{2} \mathrm{O}_{2} \rightarrow \mathrm{Fe}^{3+}+{ }^{\cdot} \mathrm{OH}+\mathrm{OH}^{-}$

where $\mathrm{Fe}^{2+}$ and $\mathrm{Fe}^{3+}$ represents the hydrated species, $\mathrm{Fe}\left(\mathrm{H}_{2} \mathrm{O}_{2}\right)_{6}{ }^{2+}$ and $\mathrm{Fe}\left(\mathrm{H}_{2} \mathrm{O}_{2}\right)_{6}{ }^{3+}$, respectively. The above reaction is often referred to as the Fenton reaction, although many other reactions occur in Fenton systems. Based on this reaction, hydroxyl radical is generated which is a nonselective and strong oxidant for the degradation of refractory pollutants. Other important reactions that may occur in aqueous mixtures of iron and hydrogen peroxide are as follows.

$\mathrm{Fe}^{3+}+\mathrm{H}_{2} \mathrm{O}_{2} \rightarrow \mathrm{Fe}^{2+}+\mathrm{HO}_{2}^{\cdot}+\mathrm{H}^{+}$

$\mathrm{Fe}^{3+}+\mathrm{HO}_{2}^{\cdot} \rightarrow \mathrm{Fe}^{2+}+\mathrm{O}_{2}+\mathrm{H}^{+}$

$\mathrm{Fe}^{2+}+\cdot \mathrm{OH} \rightarrow \mathrm{Fe}^{3+}+\mathrm{OH}^{-}$

$\mathrm{H}_{2} \mathrm{O}_{2}+{ }^{\circ} \mathrm{OH} \rightarrow \mathrm{H}_{2} \mathrm{O}+\mathrm{HO}_{2}^{\bullet}$

The iron species repetitively cycle between $\mathrm{Fe}^{2+}$ and $\mathrm{Fe}^{3+}$ as long as the hydrogen peroxide is available in the system, unless additional reactions result in formation of insoluble iron oxides and hydroxides. The presence of both inorganic and organic iron ligands in aqueous solutions can have a significant influence on the Fenton reaction. In addition to the rate of Reaction (2.7), the lifetime of free radicals can be also altered in the presence of other species resulting in changes in the $\mathrm{Fe}^{2+}$ concentration that affects the rate of formation of hydroxyl radicals.

Although Fenton did not study hydroxyl radical-mediated reactions for the combination of $\mathrm{Fe}^{3+}$ and $\mathrm{H}_{2} \mathrm{O}_{2}$, more recent work has illustrated that such systems can produce hydroxyl radical. Haber and Weiss, 1934 proposed a free radical mechanism for the combination of $\mathrm{Fe}^{3+}$ and $\mathrm{H}_{2} \mathrm{O}_{2}$ which is called as Fenton-like process (Haber and Weiss, 1934; Tarr, 2003) as shows below: 
$\mathrm{Fe}^{3+}+\mathrm{H}_{2} \mathrm{O} \rightarrow \mathrm{Fe}^{2+}+{ }^{\cdot} \mathrm{OH}+\mathrm{H}^{+}$

$\mathrm{Fe}^{2+}+\mathrm{H}_{2} \mathrm{O}_{2} \rightarrow \mathrm{Fe}^{3+}+{ }^{\cdot} \mathrm{OH}+\mathrm{OH}^{-}$

$\mathrm{Fe}^{3+}+\mathrm{HO}_{2}^{\cdot} \rightarrow \mathrm{Fe}^{2+}+\mathrm{O}_{2}+\mathrm{H}^{+}$

$\mathrm{H}_{2} \mathrm{O}_{2}+{ }^{\bullet} \mathrm{OH} \rightarrow \mathrm{H}_{2} \mathrm{O}+\mathrm{HO}_{2}^{\bullet}$

Although the treatment of pollutants with $\mathrm{H}_{2} \mathrm{O}_{2} / \mathrm{Fe}^{3+}$ has been referred to as the Haber-Weiss process (Haber and Weiss, 1934), it is apparent that the same set of reactions is involved in both the Haber-Weiss process and the Fenton process. In addition to the series of reactions in $\mathrm{H}_{2} \mathrm{O}_{2} / \mathrm{Fe}^{3+}$, some other equilibrium reactions may affect the overall reactions kinetics and mechanisms that are listed as follows.

$\mathrm{H}_{2} \mathrm{O}_{2} \rightleftharpoons \mathrm{H}^{+}+\mathrm{HO}_{2}^{-}$

$F e^{2+}+n L^{-m} \rightleftharpoons\left(F e L_{n}\right)^{2-m \times n}$

$F e^{3+}+n L^{-m} \rightleftharpoons\left(F e L_{n}\right)^{3-m \times n}$

where $L$ represents a ligand. Common ligands may include $\mathrm{OH}^{-}, \mathrm{Cl}^{-}, \mathrm{HCO}_{3}^{-}, \mathrm{PO}_{4}^{3-}, \mathrm{NO}_{2}^{-}$, and $\mathrm{SO}_{4}^{2-}$. The coordination by anionic ligands makes oxidation of $\mathrm{Fe}^{2+}$ easier, yielding an increase in rate constant of Reaction (2.7). On the contrary, coordination of $\mathrm{Fe}^{3+}$ by anions may be more difficult to reduce to the divalent species. Such changes in oxidation/reduction potentials for $\mathrm{Fe}^{2+}$ and $\mathrm{Fe}^{3+}$ not only change the rate constant of Reaction (2.7), but also change the relative concentrations of the two iron species resulting in the change of the rates of several reactions in iron/peroxide systems. Iron hydroxides and oxyhydroxides are likely to precipitate under higher 
$\mathrm{pH}$ conditions. As a result, Fenton processes generally must be carried out at $\mathrm{pH}$ values well below 7, unless iron-solubilizing agents (chelators) are added.

Additional reactions may also take place in photo-Fenton and photo-Fenton-like process as follows (Pignatello et al., 1999):

$\mathrm{H}_{2} \mathrm{O}_{2} \stackrel{h v}{\rightarrow} 2 \cdot \mathrm{OH}$

$F e^{3+}\left(L^{-}\right) \stackrel{h v}{\rightarrow} F e^{2+}+L^{\bullet}$

The efficiency of the photo-Fenton or photo-Fenton like processes is higher than that ofthe dark processes. Although there is still a debate as to whether hydroxyl radicals or ferryl species are the key oxidants in Fenton systems, most research reports the hydroxyl radicals are responsible for the degradation of organic compounds (Tarr, 2003). Therefore, any additional route to reduce

$\mathrm{Fe}^{3+}$ to $\mathrm{Fe}^{2+}$ enhances the production rate of hydroxyl radical based on Reaction (2.20). Other additional reactions (including other anion radicals) may also need to be considered according to the specific conditions.

\subsubsection{Kinetic Modeling of AOTs}

Kinetic modeling allows predictions of the performance of any reacting system and provides an efficient tool for process design. In fact, the main objective of kinetic modeling is to enable us to predict beforehand the rate at which given chemical substances react and to control the rate in some desirable fashion. The kinetic expressions are developed based on a reliable reaction mechanism showing the step-by-step sequence of elementary processes and reaction intermediates by which the overall chemical change takes place. In general, a photochemical 
reaction will proceed in a single molecular pathway that involves several elementary steps. Normally, the majority of them are dark reactions while, ordinarily, one activation step is produced by radiation absorption of a reactant molecule or a catalyst. From the kinetics point of view, dark reactions do not need a different methodological approach than conventional reactions. Conversely, the activation step constitutes a distinctive aspect. The rate of the radiation activation step is proportional to the absorbed energy via a property that is defined as the local volumetric rate of energy absorption (LVREA) that will be discussed in the next section. Hydroxyl radicals which are the main cause of degradation react rapidly with a wide array of species. In general, reactions follow second-order kinetics. In many cases, aqueous reactions with hydroxyl radicals are diffusion-limited. A comprehensive source for the second-order rate constant of reactions with hydroxyl radicals is available (Buxton et al., 1988). Most compounds that have been studied have rate constants between $10^{5}$ and $10^{10} \mathrm{M}^{-1} \mathrm{sec}^{-1}$, but a few compounds with lower rate constants have been reported. Therefore, kinetic models employ a set of differential equations based on the mole balances of the main species involved in the process. Solving this system of ordinary differential equations, results in finding the concentration profile of each species with time. In order to validate the theoretical results, they can be compared to the experimental data once they are available. Also, the rate constants could be determined using optimization algorithms or trial and error procedures. These procedures result in finding the best values that fit the experimental and calculated concentrations.

\subsubsection{Kinetics of Photolysis}

As mentioned earlier, the initiation or activation step in a photochemical reaction is the radiation absorption by a molecule or a catalyst. The rate of radiation absorption could be determined 
through the LVREA. The LVREA characterizes the amount of photons that are absorbed per unit time and unit reaction volume. The methodology to treat the activation step could be understood better considering the typical approach in chemical reaction engineering. Figure 2.4 estimates the LVREA. The first step is writing the mass balance in which the reaction rates for all participating species are formulated in the form of differential equations. For the activation step, the kinetic expression necessarily involves the photon absorption rate (LVREA). The kinetics of activation reactions is highly dependent on several factors such as light intensity, the wavelength of the incident radiation, the optical path of the radiation, the nature of the compound irradiated and the solution in which it is present. The performance of UV radiation will also depend on the photoreactor design. For example, in a batch photochemical reactor, the rate of compound removal due to direct photolysis assuming the mechanism of Reactions (2.21) to (2.23) is as follows (Tarr, 2003, Beltrán et al., 1995):

$M+h v \stackrel{k_{a}}{\rightarrow} M^{*}$

$M^{*} \stackrel{k_{b}}{\rightarrow} M$

$M^{*} \stackrel{k_{c}}{\rightarrow}$ Products

$r_{U V}=\frac{\mathrm{d} C_{M}}{\mathrm{~d} t}=-\frac{1}{V} \int_{V} k_{a}\left[1-\frac{k_{b}}{k_{b}+k_{c}}\right] F_{M} \mu q_{i} \mathrm{~d} V$

where $C_{M}$ is the concentration of compound $M, V$ is the reaction volume, $k_{a}, k_{b}$, and $k_{c}$ are the rate constants of the Reactions (2.21) to (2.23), $q_{i}$ is the flux of incident radiation and $F$ is the fraction of radiation, defined in Equation (2.25), that is absorbed by $M$ (Tarr, 2003).

$F_{M}=\frac{\varepsilon_{M} C_{M}}{\sum \varepsilon_{i} C_{i}}$ 


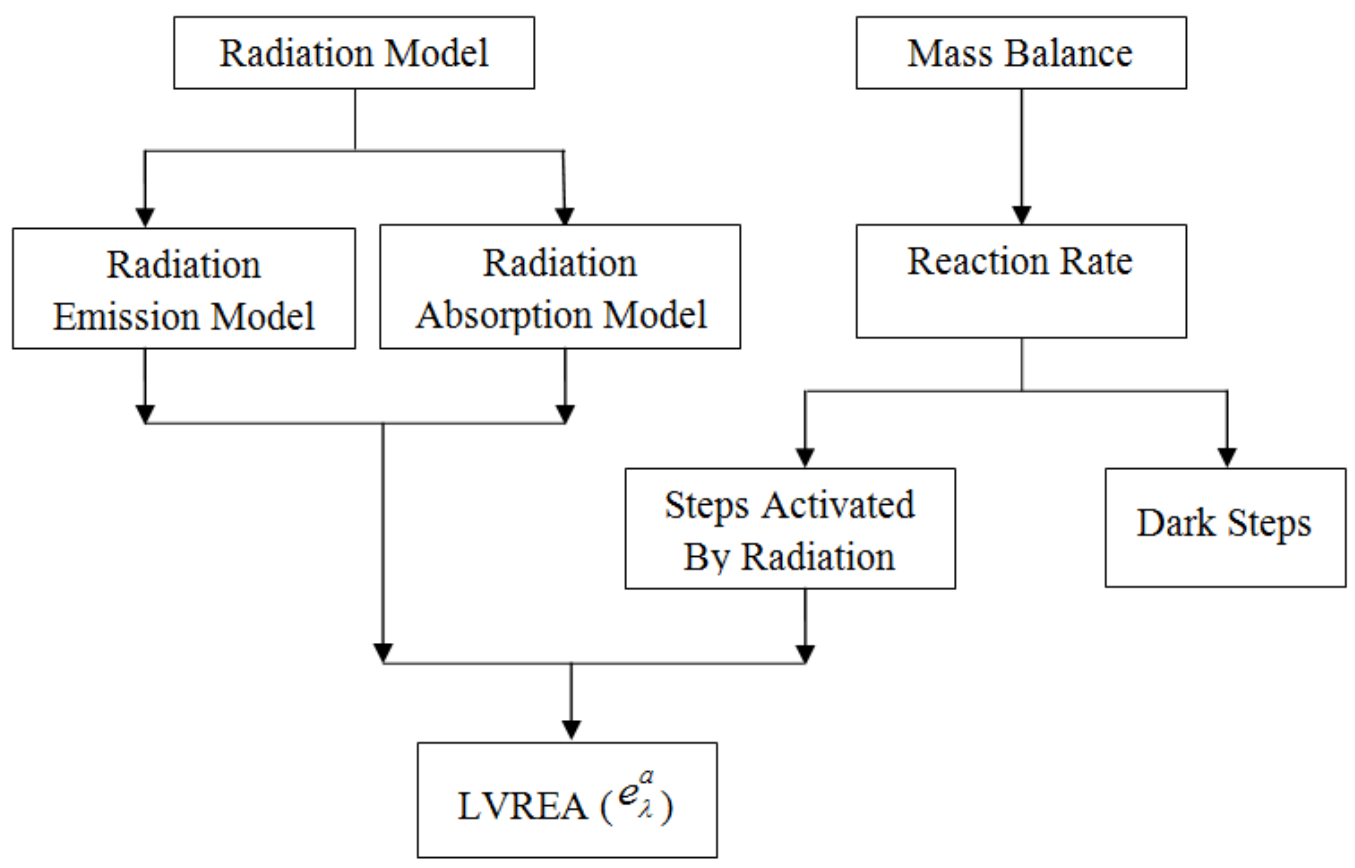

Figure 2.4. Flowchart of evaluation of local volumetric rate of energy absorption (LVREA). 
where $\varepsilon_{M}$ and $\varepsilon_{i}$ are the molar absorption coefficient of $M$ and any compound $i$ present in solution that absorbs radiation. Also, $\mu$ which is the function of extinction coefficient of species present in water, $\varepsilon_{i}$, could be defined as follows:

$\mu=2.303 \sum \varepsilon_{i} C_{i}$

In general, modeling of a photoreactor requires evaluation of LVREA by the reactants that undergo photolysis. For a homogeneous photoreactor, the LVREA is evaluated by solving a radiation energy balance for a homogeneous control volume and a radiation source model with the absorption term represented by an absorption constitutive equation. Therefore, the choice of the radiant energy source model is a significant issue that must be taken into account. Two different groups of source models have been developed to predict radiation profiles for different types of photoreactors: (i) incidence models, and (ii) emission models (Alfano et al., 1986). The former one generates a mathematical model assuming the existence of a given radiant energy distribution in the vicinity of the reactor and the latter proposes a model for the source emission. The emitting source in the emission models can be either a line source (Jacob and Dranoff, 1966) which solves the problem considering the lamp as a line source or it could be a three-dimensional source that models the extense source as a volume with three dimensions with the emission considered either as extense source model with volumetric emission (ESVE model) (Irazoqui et al., 1973) or extense source model with superficial emission (ESSE model) (Stramigioli et al., 1977). In ESVE and ESSE, the lamp radius is considered as an additional parameter in the design of a photochemical reactor. For the purpose of modelling photoreactors for water purification, an ESVE model may be used if a UV arc source lamp is used, but an ESSE model would better represent germicidal or fluorescent lamps (Cerdá et al., 1978; Alfano et al., 1986). Using the ESVE model, a three-dimensional radiation balance equation is obtained considering the spatial 
relationship between the light source which gives volumetric (three-dimensional) emission and any point in space within the reactor. The evaluation of the LVREA could be performed by stating the radiative transfer equation (RTE) that needs the appropriate constitutive equations for radiation absorption, emission, and scattering (Cassano et al., 1995). The RTE is an integrodifferential equation that involves all the constitutive equations for the comprised physical phenomena and the appropriate boundary conditions. For the participating media, the constitutive equations involve the physical properties that depend on each special case: absorption and scattering coefficients. Consequently, the RTE will be defined that could be applied to each photoreactor configuration. The RTE is derived for a single propagation direction and a single wavelength. In order to write a photon balance in a bounded volume, the spectral specific intensity has to be defined. This property is expressed as the amount of energy $(E)$ per unit cross section area perpendicular to $\Omega(\Delta A \cos \theta)$, per unit time $(t)$, per unit solid angle $(\Omega)$, and per unit wavelength $(\lambda)$ interval as follows:

$I(x, t)=\lim _{(\Delta A, \Delta \Omega, \Delta t, \Delta \lambda) \rightarrow 0}\left(\frac{\Delta E_{\lambda}}{\Delta A \cos \theta \Delta t \Delta \lambda}\right)$

where $I$ is the radiation intensity (Einstein $\mathrm{s}^{-1} \mathrm{~m}^{-2} \mathrm{sr}^{-1}$ ). As it is shown, the monochromatic radiation intensity $(\lambda)$ is a function of position $(x)$, direction $(\Omega)$, and time $(t)$. Therefore, the complete definition involves three spatial coordinates, two directional coordinates (in spherical system), and a temporal coordinate. This property that is used to formulate the RTE is considered as the starting point to calculate the incident radiation at any material point inside a reaction space. Using the defined spectral specific intensity, the photon balance in a bounded volume could be written as follows (Cassano et al., 1995): 


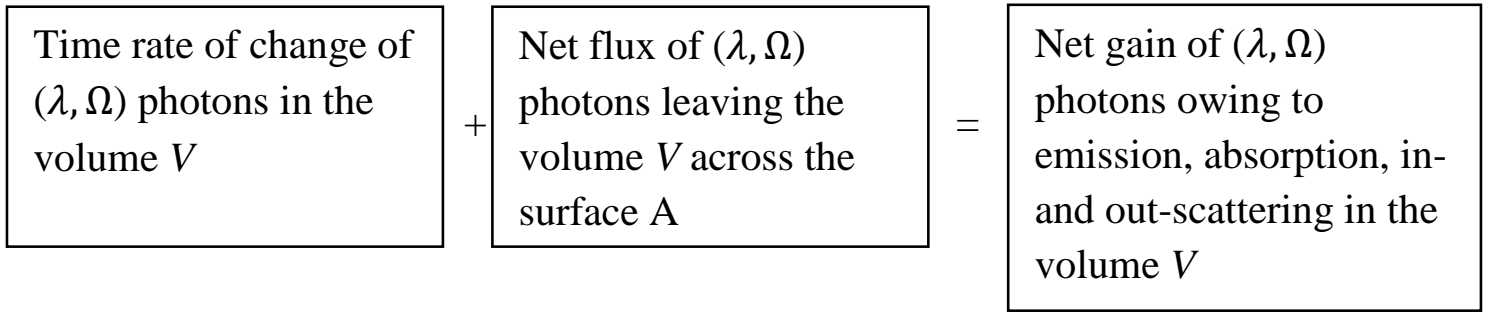

This fundamental law can be mathematically formulated considering the two source terms (emission and in-scattering) and the two sink terms (absorption and out-scattering) on the righthand side of the above expression:

$$
\frac{1}{c} \cdot \frac{\partial I_{\lambda, \Omega}}{\partial t}+\nabla \cdot\left(I_{\lambda, \Omega}\right)=W_{\lambda, \Omega}^{\text {em }}+W_{\lambda, \Omega}^{\text {abs }}+W_{\lambda, \Omega}^{\text {out-scatt. }}+W_{\lambda, \Omega}^{\text {in-scatt. }}
$$

where $c$ and $t$ are the light speed and time and considering the magnitude of the light speed the first term could be neglected. Since practical cases will be usually operated at room temperatures, internal emission can be safely ignored $\left(W_{\lambda, \Omega}^{e m}=0\right)$.

Equation (2.29) is the general formulation of a radiation balance for a single direction and a given amount of transported energy. However, it is not very useful as it does not have sufficient information to evaluate the terms in its right-hand side. This problem is solved by introducing a constitutive equation for each term. This can be performed by the measurement of the perceptible behaviour of each phenomenon and its relationship with the involved observable physical properties affecting each of the terms. Therefore, the RTE could be written in the following form:

$$
\frac{\mathrm{d} I_{\lambda, \Omega}}{\mathrm{d} s}(s, t)+\underbrace{k_{\lambda}(s, t) I_{\lambda, \Omega}(s, t)}_{\text {Absorption }}+\underbrace{\sigma_{\lambda}(s, t) I_{\lambda, \Omega}(s, t)}_{\text {Out-scattering }}=\underbrace{\frac{\sigma_{\lambda}(s, t)}{4 \pi} \int_{\Omega^{\prime}=4 \pi} p_{\lambda}\left(\Omega^{\prime} \rightarrow \Omega\right) I_{\lambda, \Omega}(s, t) \mathrm{d} \Omega^{\prime}}_{\text {In-scattering }}
$$


where $k_{\lambda}(s, t)$ is the linear or volumetric absorption coefficient representing the fraction of incident radiation that is absorbed by the matter per unit length along the path of the beam $\left(\mathrm{m}^{-1}\right)$. $\sigma_{\lambda}(s, t)$ is the linear or volumetric scattering coefficient representing the fraction of the incident radiation that is scattered by the matter in all directions and in other frequencies, per unit length of the path of the beam $\left(\mathrm{m}^{-1}\right)$, and $p_{\lambda}$ is the phase function (dimensionless). Therefore, this equation could be applied only to a medium that may be considered as pseudo-homogeneous. This restriction may put some limits on the application of this equation on heterogeneous media. In other words, in the case of particle sizes bigger than $0.5-1 \mathrm{~mm}$ and concentration of solids higher than 1-2\% (heterogeneous media) a different approach has to be considered.

\subsection{PHOTOCHEMICAL DEGRADATION OF POLYMERS}

A necessary condition for a photochemical reaction to take place is the excitation of a molecule by the absorption of light quanta of sufficient energy. This is the well known 'Grotthus-Draper Law' (Ranby and Rabek, 1975). Completely saturated compounds do not absorb light at higher wavelengths of $330 \mathrm{~nm}$. Only double bonds reveal strong radiation absorption in the UV range. Since most polymers contain $\mathrm{C}-\mathrm{C}, \mathrm{C}-\mathrm{H}, \mathrm{C}-\mathrm{O}, \mathrm{C}-\mathrm{N}$, and $\mathrm{C}-\mathrm{Cl}$ bonds, they are not expected to absorb light at wavelengths higher than $190 \mathrm{~nm}$. The fact that many polymers absorb light and form free radicals with UV light even at the wavelength longer than $300 \mathrm{~nm}$ indicates the presence of chromophores in these polymers. Chromophores are a system of double bonds which shift the absorption spectrum towards visible range. Therefore, the presence of chromophores and also impurities (such as carbonyl groups) which can be formed during synthesis or the processing of polymers are likely responsible for the UV absorption. Carbonyl groups, when 
exposed in the range of $270-330 \mathrm{~nm}$, are easily excited to singlet and triplet states which could subsequently initiate some of photochemical reactions (Ranby, 1989).

The photochemical reactions of excited carbonyl groups in organic compounds are classified as Norrish reactions of type I and II (Ranby and Rabek, 1975) as explained below:

(i) Type I Norrish's reaction: In the primary process, the bond between the carbonyl group and adjacent $\alpha$-carbon is homolytically cleaved. Two possible primary reactions can occur:

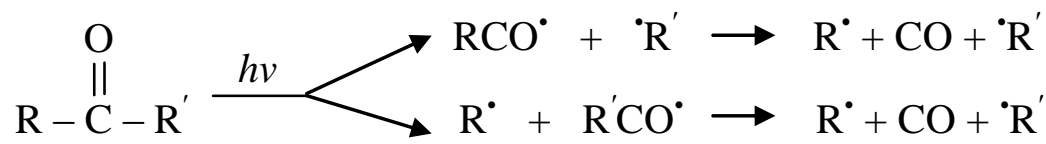

(ii) Type II Norrish's reaction: A nonradical, intermolecular process which occurs with the formation of a six-membered cyclic intermediate. The abstraction of a hydrogen from the $\gamma$ carbon results in its subsequent decomposition into an olefin and an alcohol or an aldehyde:

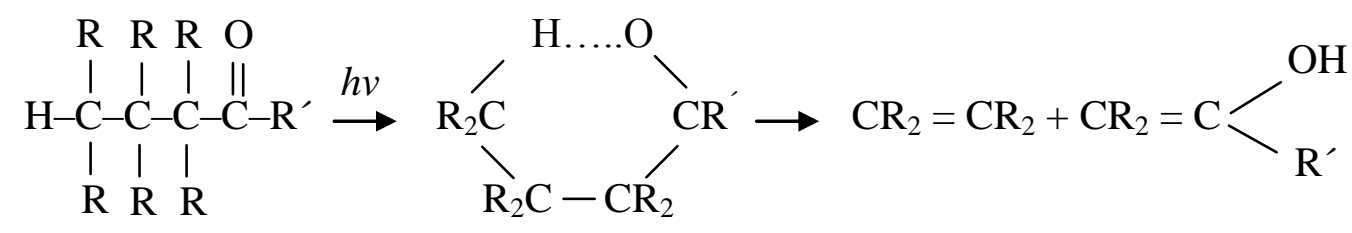

\subsubsection{General Mechanism of Photodegradation of Polymers}

Photochemical reactions in polymers usually take place in two stages (Reich and Stivala, 1971):

(i) The primary photochemical reaction which refers to the excitation of a molecule directly due to the absorbed light quantum.

(ii) Secondary ('dark') reactions of the various chemical species such as molecules, ions, or radicals produced by the primary photochemical reaction. 
Most synthetic polymers are amenable to degradation initiated by ultraviolet and visible light. The UV light quanta (400-100 nm) have energies from 3.1 to $12.4 \mathrm{eV}$ which corresponds to 72 $286 \mathrm{kcal} / \mathrm{mol}$, respectively. This amount of energy is sufficient for breaking most common chemical bonds. For example bond dissociation energies are $94-99 \mathrm{kcal} / \mathrm{mol}$ for $\mathrm{C}-\mathrm{H}$ bonds in alkenes, $77-83 \mathrm{kcal} / \mathrm{mol}$ for $\mathrm{C}-\mathrm{C}$ bonds in aliphatic compounds, and $76-79 \mathrm{kcal} / \mathrm{mol}$ for $\mathrm{C}-\mathrm{O}$ bonds in aliphatic ethers. The mechanisms of the photodegradation and oxidation reactions, quite well known in principle, are as follows (Ranby and Rabek, 1975):

- Light quanta are absorbed by external impurities and/or internal chromophoric groups (carbonyl and phenyl) in the polymer and form excited states, initially singlets (shortlived) which are transformed to triplets (long-lived and reactive).

- Excited triplet states may cleave the polymer chains and form radical pairs (Norrish Type I reaction) or form pairs of saturated and unsaturated chain ends by hydrogen abstraction (Norrish Type II reaction).

- The polymer radicals formed $\left(\mathrm{P}^{*}\right)$ react with molecular oxygen (in triplet ground state) to form peroxy radicals $\left(\mathrm{PO}_{2}{ }^{\circ}\right)$ which abstract hydrogen and form hydroperoxide groups $\left(\mathrm{PO}_{2} \mathrm{H}\right)$.

- The hydroperoxide groups $\left(\mathrm{PO}_{2} \mathrm{H}\right)$ absorb UV light, then the weak $\mathrm{O}-\mathrm{O}$ bonds break and pairs of alkoxy $\left(\mathrm{PO}^{\circ}\right)$ and hydroxyl radicals $\left({ }^{\circ} \mathrm{OH}\right)$ are formed which may react in different ways such as hydrogen abstraction, chain scission, and rearrangement. This step results in bond scission and subsequently photodegradation.

- Recombination and crosslinking of free radicals result in termination of the chain.

The general mechanism of photodegradation of polymers mentioned above are summarized in Figure 2.5 where the polymer is represented by $\mathrm{PH}$. According to the reaction scheme of the 
photooxidation of polymers, the mechanism of polymer degradation can be described by the following simple sequences:

Initiation: $\quad \mathrm{PH} \stackrel{h v\left(\mathrm{O}_{2}\right)}{\longrightarrow} \mathrm{P}^{\cdot}+\mathrm{HO}_{2}^{\cdot}$

Propagation:

$$
\mathrm{P}^{\cdot}+\mathrm{O}_{2} \longrightarrow \mathrm{PO}_{2}^{\bullet}
$$

$$
\mathrm{PO}_{2}^{\bullet}+\mathrm{PH} \longrightarrow \mathrm{POOH}+\mathrm{P}^{\bullet}
$$

$$
\mathrm{POOH}+\mathrm{PH} \longrightarrow \mathrm{P}=\mathrm{O}+\mathrm{H}_{2} \mathrm{O}+\mathrm{P}^{\bullet}
$$

Chain branching: $\quad \mathrm{POOH} \stackrel{h v}{\longrightarrow} \mathrm{PO}^{\cdot}+{ }^{\circ} \mathrm{OH}$

Degradation: $\left\{\begin{array}{l}\text { (B-Scission) } \\ \text { (Norrish type I) }\end{array}\right.$

Termination: $\left\{\begin{array}{l}\mathrm{PO}^{\cdot}+\mathrm{PH} \longrightarrow \mathrm{POH}+\mathrm{P}^{\cdot} \\ \mathrm{HO}^{\bullet}+\mathrm{PH} \longrightarrow \mathrm{H}_{2} \mathrm{O}+\mathrm{P}^{\cdot} \\ \mathrm{P}^{\cdot}+\mathrm{P}^{\cdot} \longrightarrow \mathrm{P}-\mathrm{P} \\ \mathrm{PO}_{2}^{\cdot}+\mathrm{PO}_{2}^{\cdot} \longrightarrow \mathrm{POOP}+\mathrm{O}_{2} \\ \mathrm{PO}_{2}^{\cdot}+\mathrm{PO}_{2}^{\cdot} \longrightarrow \mathrm{P}=\mathrm{O}+\mathrm{POH}+\mathrm{O}_{2} \\ \mathrm{PO}^{\cdot}+\mathrm{PO}_{2}^{\cdot} \longrightarrow \mathrm{POP}+\mathrm{O}_{2}\end{array}\right.$ 


\subsubsection{Types of Polymer Chain Scission}

Broadly, the degradation of polymers may be considered any type of modification of a polymer chain involving the main-chain backbone or side groups or both due to chemical, physical, or biological reactions. These modifications which are often of a chemical nature result in main chain scission and subsequent chemical transformations which can lead to lower molecular weights, crosslinking, and cyclization. The main chain scission may occur in one of two ways, or both, corresponding approximately to the reverse of the two types of polymerization processes: condensation and addition polymerization (Reich and Stivala, 1971). Thus, the scission may occur randomly (random degradation) where chain breaking occurs at random points along the chain or at the terminal of a polymer radical or polymer where monomer units are successfully released (unzipping). In the former case, which is the reverse of condensation polymerization, the molecular weight decreases continuously with the extent of reaction. Therefore, the fragments formed in random scission which are large relative to monomer units, are mixtures having molecular weights up to several hundred. In the latter form of depolymerization, the production of monomer is appreciable. This case is the reverse of the propagation step in vinyl polymerization. There is also a special case that the scission takes place at the midpoint of the chain which is mainly due to the mechanical or ultrasonic degradation. Scission and crosslinking may occur simultaneously in the same polymer. This scission may turn a solid polymer into a thin liquid and crosslinking may create a brittle polymer network that is insoluble and infusible.

Generally, the competition ensues between the two types of reaction mechanisms. On the other hand, the breaking of secondary valence bonds within a chain, e.g., hydrogen bonds in proteins, leads to conformational changes which constitute a degradative process called denaturation, influenced by heat, $\mathrm{pH}$ changes, chemical agents, etc. 


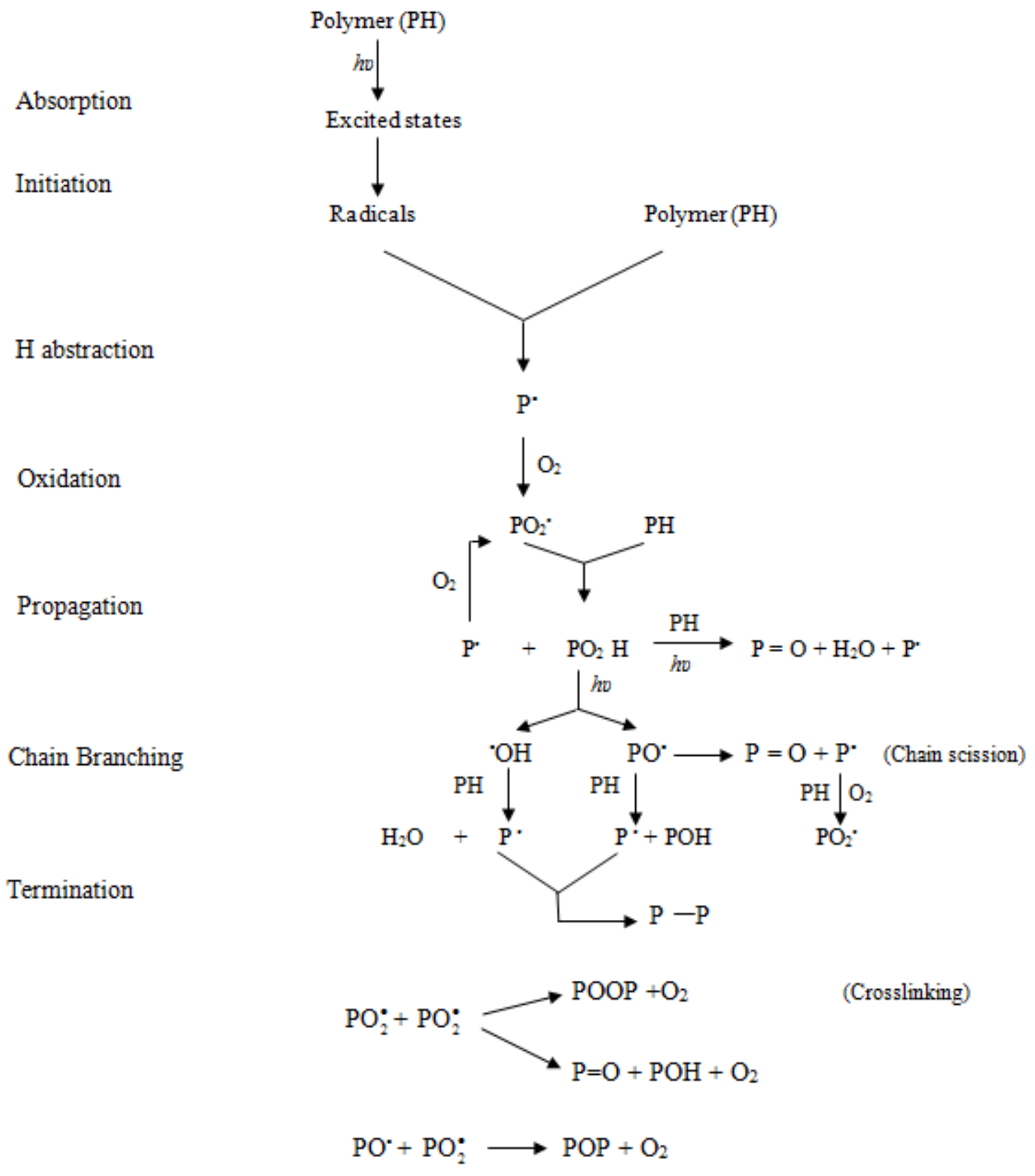

Figure 2.5. General mechanism of photodegradation of polymers. 
Here, the main concern is the degradative process which affects the breaking of primary valence bonds, essentially in the chain backbone, accompanying by a decrease in the polymer molecular weight.

\subsubsection{Kinetic Modeling of Polymer Degradation}

Kinetic modeling is one of the most efficient tools to unravel the mechanistic chemistry directing to polymer degradation. On the other hand, achieving a valid kinetic model based on a known and well-established reaction mechanism is considered as the best starting point for design purposes. According to the reaction mechanisms, equations representing the evolution of reactions could be derived.

As mentioned earlier, polymer degradation is considered as any type of change of a polymer chain involving the main-chain backbone or side groups or both. These changes in aqueous solution result in the main chain scission and subsequently, lower molecular weights. The photodegradation of polymers has been studied in different aspects resulting in generation of compounds of lower molecular weight (Iordan et al., 2004; Kaczmarek et al., 2004; Santos et al., 2005; Carroccio and Puglisi, 2005; Suji et al., 2006; Guang-meng et al., 2006; Ani and Ramadhan, 2008; Giroto et al., 2008; Martins-Franchetti et al., 2008; Shukla et al., 2009; Roy et al., 2009; Konaganti and Madras, 2010). Therefore, most investigations in polymer degradation have centered on determining the monomer yield and the rate of change of average molecular weight. In the next section, based on the kinetic mechanisms, the dynamics of polymer degradation are described. 


\subsubsection{Population-balance equations}

The degradation of polydisperse high molecular weight materials results in the formation of thousands of polymeric chains with different chain lengths and various chemical compositions, i.e., the number of branches, the presence and the position of a radical center, and the location of double bonds. Tracking each of these distinct species individually is not possible and due to current hardware limitations, models with the implied number of equations are not easy to solve. To overcome these difficulties, population-balanced based models have been developed to study molecular weight changes and small molecule evolution simultaneously (Madras et al., 1995; Wang et al., 1995; Kodera and McCoy 1997; McCoy and Madras, 1997; Sezgi et al., 1998). Population-balance equations (PBEs) have been established in several branches of modern science, mainly in branches with particulate entities such as crystallization and polymerization. PBEs describe how populations of separate entities develop in specific properties over time. Therefore, they belong to a subcategory of equations known as partial differential equations. Population-balance equations are often applied in fragmentation models to explain how the frequency distributions of different-size entities evolve.

As polymer degradation is a fragmentation phenomenon, continuous-distribution mass (population) balances could be applied to study the kinetics of the polymer degradation. Rate expressions are typically based on free radical mechanisms (Simha and Wall, 1958). A free radical mechanism evaluates rate coefficients based on continuous-distribution kinetics which provide more details of the degradation process by accounting the time dependence of the complete molecular weight distributions (MWDs). The MWD is a partial record of the kinetics and mechanisms that influences its evolution and contains much more information than the lumped concentration. 
Broadly, the reaction mechanism often used to describe the degradation of macromolecules comprises three reversible elementary steps: initiation-termination of radicals, hydrogen abstraction (Rice and Herzfeld, 1939; Kodera and McCoy, 1997), and depropagation (scission, decay). As mentioned earlier, the chain-end scission is considered as the main mechanism of the photodegradation. According to the long chain approximation (LCA), initiation-termination steps are neglected because of the insignificant effect on the overall reaction rates (Gavalas, 1966; Nigam et al., 1994).

Therefore, the important elementary reactions of photooxidative degradation of polymers can be classified in three different ways: transformation without change in molecular weight, chain scission, and combination to form higher molecular weight compounds. These reactions can be incorporated in a mathematical model based on continuous-distribution kinetics. Therefore, the kinetic model is constituted by the following four reactions:

(i) Intermolecular hydrogen abstraction that involves an intermediate macromolecular complex represented by the following reaction (Smagala, 2003):

$R_{n}^{\bullet}+P_{n^{\prime}} \rightarrow I\left(n+n^{\prime}\right) \rightarrow P_{n}+R_{n^{\prime}}^{\bullet}$

where $P_{n}$ and $P_{n^{\prime}}$ are the polymer molecules of $n$ and $n^{\prime}$ molecular weights, $R_{n}^{\bullet}$ and $R_{n^{\prime}}^{\cdot}$ are the polymer radicals, and $I$ is the intermediate product. Because the intermediate product $\left(I\left(n+n^{\prime}\right)\right)$ is short-lived, effectively $R_{n}^{\bullet}$ is converted to $P_{n}$ and $P_{n^{\prime}}$; and the polymer molecule of molecular weight $n^{\prime}$, is converted to $R_{n^{\prime}}^{\cdot}$. Thus, the reaction can be written as:

$$
P_{n} \underset{k_{H}}{\stackrel{k_{h}}{\rightleftharpoons}} R_{n}^{\bullet}
$$


where $k_{h}$ and $k_{H}$ represent rate constants for reversible hydrogen abstraction from a polymer to form an end radical. The MW of the hydrogen atom is considered negligible in comparison to the MW of the macromolecule and the reaction rate is pseudo-first order (Kodera and McCoy, 1997).

(ii) Propagation-depropagation for random chain scission:

$R_{n}^{\cdot} \stackrel{k_{d}}{\rightarrow} R_{n-n^{\prime}}^{\cdot}+P_{n^{\prime}}$

where $k_{d}$ represents the degradation coefficient for the depropagation of the random radical.

(iii) Interamolecular hydrogen abstraction in the chain-end scission:

$R_{n}^{\bullet} \underset{k_{H e}}{\stackrel{k_{h e}}{\rightleftharpoons}} R_{S}^{\bullet}$

where $k_{h e}$ and $k_{H e}$ represent rate constants for reversible hydrogen abstraction from a polymer to form an end radical $\left(R_{S}^{*}\right)$.

(iv) Propagation-depropagation for chain-end scission that a specific product is formed via specific (end) radical according to the Rice-Kossiakoff mechanism (Kossiakoff and Rice, 1943).

$R_{s}^{\cdot}(n) \stackrel{k_{s}}{\longrightarrow} Q_{s}\left(n_{s}\right)+R_{S}\left(n-n_{s}\right)$

where $k_{s}$ represents the degradation coefficient for the depropagation of $\beta$-scission of end radical. The $\beta$-scission is the most important cracking reaction in which a bond in $\beta$ position (second carbon next to a functional group) breaks to give an olefin and another free radical. As mentioned earlier, the hydrogen abstraction from the polymer of the chain length $n$ results in the 
formation of radicals of the same chain length $\left(R_{n}^{\bullet}\right)$. The binary degradation of $R_{n}^{\bullet}$ could be represented by the following sequential reactions:

$$
\begin{aligned}
& R_{n}^{\bullet} \rightarrow\left(R_{n}^{\bullet}-R_{n-1}^{\bullet}\right)+R_{n-1}^{\bullet} \\
& R_{n-1}^{\bullet} \rightarrow\left(R_{n-1}^{\bullet}-R_{n-2}^{\bullet}\right)+R_{n-2}^{\bullet} \\
& \vdots \\
& R_{2}^{\bullet} \rightarrow\left(R_{2}^{\bullet}-R_{1}^{\bullet}\right)+R_{1}^{\bullet}\left(M_{1}\right)
\end{aligned}
$$

which, in turn, could be written as follows:

$R_{n}^{\bullet} \rightarrow\left(R_{n}^{\bullet}-R_{n-1}^{\bullet}\right)+\left(R_{n-1}^{\bullet}-R_{n-2}^{\bullet}\right)+\cdots+\left(R_{2}^{\bullet}-R_{1}^{\bullet}\right)+R_{1}^{\bullet}\left(M_{1}\right)$

Generally, for a binary fragmentation reaction, the product kinetics is represented by Equation (2.56) in which the integral is used instead of the sum to make the calculations easier (McCoy and Wang, 1994):

$R(n)=\int_{n}^{\infty} k\left(n^{\prime}\right) \Omega\left(n, n^{\prime}\right) r\left(n^{\prime}, t\right) \mathrm{d} n^{\prime}$

where $k\left(n^{\prime}\right)$ and $r\left(n^{\prime}, t\right)$ are the rate constant of the binary scission and the chain length distribution of the polymer radical, respectively. The stoichiometric coefficient $\Omega(n, n)$ represents the likelihood of obtaining a scission product of the chain length $n$ and $n^{\prime}-n$ from a larger molecule $n^{\prime}$. The stoichiometric coefficient satisfies the normalization and symmetry conditions as follows.

$\int_{0}^{n^{\prime}} \Omega\left(n, n^{\prime}\right) \mathrm{d} n=1$

$\Omega\left(n, n^{\prime}\right)=\Omega\left(n^{\prime}-n, n^{\prime}\right)$ 
A general expression for the stoichiometric coefficient, or kernel, is given by the following expression (McCoy and Wang, 1994):

$\Omega\left(n, n^{\prime}\right)=\frac{n^{m}\left(n^{\prime}-n\right)^{m} \Gamma(2 m+2)}{\Gamma(m+1)^{2}\left(n^{\prime}\right)^{2 m+1}}$

where $\Gamma$ is the gamma function and $m$ is a parameter indicating the shape of the scission fragment distribution. The stoichiometric coefficient is plotted in Figure 2.6 for several values of $m$. For $m=1$ the kernel reduces to the quadratic form for polymer degradation (Ziff and McGrady, 1986) as follows:

$$
\Omega\left(n, n^{\prime}\right)=\frac{6 n\left(n-n^{\prime}\right)}{n^{\prime 3}}
$$

And when $m=0$ (random chain scission), the products are evenly distributed along all $n \leq n^{\prime}$ as follows:

$\Omega\left(n, n^{\prime}\right)=\frac{1}{n^{\prime}}$

$m \rightarrow \infty$ represents the midpoint chain scission as follows:

$\Omega\left(n, n^{\prime}\right)=\delta\left(n-\frac{n^{\prime}}{2}\right)$

Therefore, the population balance equations for the polymer and the polymer radical undertaking random chain scission can be written as follows:

$$
\begin{aligned}
& \frac{\mathrm{d} p}{\mathrm{~d} t}=-k_{h} p(n)+k_{H} p(n)+k_{d} \int_{x}^{\infty} r\left(n^{\prime}\right) \Omega\left(n, n^{\prime}\right) \mathrm{d} n^{\prime} \\
& \frac{\mathrm{d} r}{\mathrm{~d} t}=k_{h} p(n)-k_{H} p(n)-k_{d} r(n)+k_{d} \int_{x}^{\infty} r(n) \Omega\left(n, n^{\prime}\right) \mathrm{d} n^{\prime}
\end{aligned}
$$


Equations (2.63) and (2.64) are integro-differential equations that are not easy to solve. Two approaches could be considered to solve this type of equations as it is presented in the next section.

\subsection{Solution methods}

The first approach to solve the integro-differential equation is classified as sectional methods. Using the sectional methods one can simulate the evaluation of the entire population density profile over time. However, high computational cost is one of the drawbacks of this method. The second approach is applying the so-called moment operations to overcome the large computational cost in the sectional method. In this method, the simulation is performed using the finite number of moments to describe the population density profile.

\subsection{Sectional Method}

In this method, a finite-difference expression is applied for the derivative to solve the population balance equations. In order to solve the integral part, the midpoint integration is used. For example for the following simple random chain scission, the population balance equation could be written by Equation (2.65).

$P_{n} \stackrel{k}{\rightarrow} P_{n^{\prime}}+P_{n-n^{\prime}}$

$\frac{\mathrm{d} p}{\mathrm{~d} t}=-k p_{n}+2 k \int_{n}^{\infty} p\left(n^{\prime}\right) \mathrm{d} n^{\prime}$

By applying the finite-difference method, the discrete form of Equation (2.66) could be written as follows. 


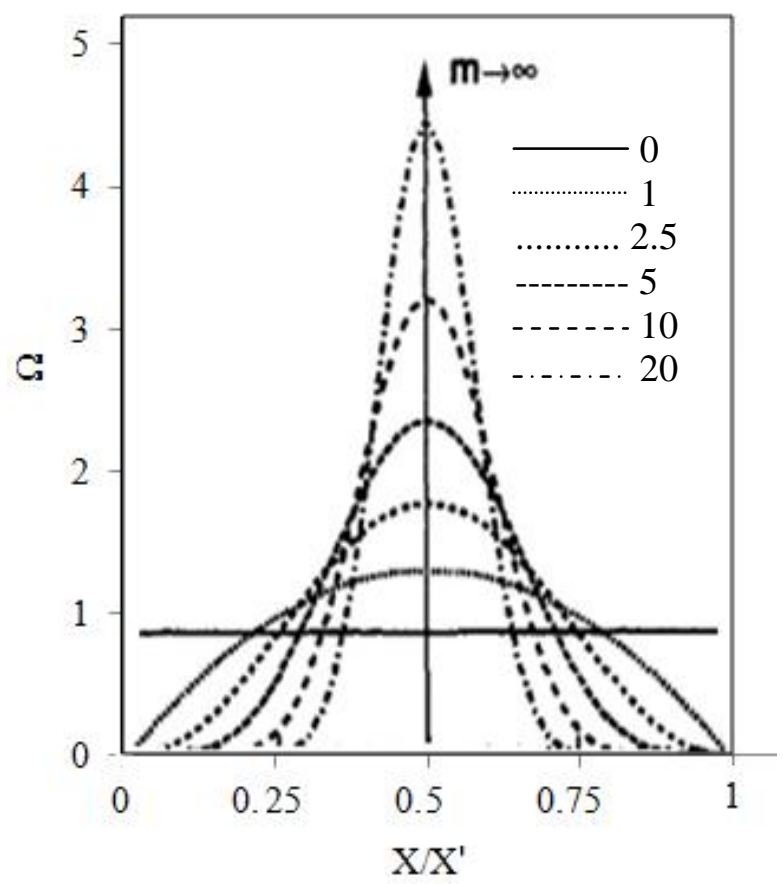

Figure 2.6. Stoichiometric coefficient (kernel) for several values of $m$. (Adapted by McCoy and Madras, 1997). 
$p_{j+1, i}=p_{j, i}+\Delta t\left[-k p_{j, i}+2 k \sum_{k=i}^{N-1} p_{j, k} \Delta x\right]$

Applying this method generates a solution in which mass is not conserved. This points out that the midpoint quadrature is not precise enough to resolve the integral term it approximates (Bruns et al., 2009). Therefore, in order to overcome this problem, other integration schemes have to be proposed that make the problem more complicated. In the next section, the moment operation is introduced as an easier method to transform the integro-differential equations to ordinary differential equations.

\subsection{Moment Method}

Any density distribution function could be modeled in terms of its moment. A small number of moments is usually adequate to characterize a distribution. The moments of the MWDs $\left(p^{(p)}(t)\right)$ could be expressed as the integrals over the MW, $x$, as follows:

$p^{(p)}(t)=\int_{0}^{\infty} p(t, n) n^{p} \mathrm{~d} n$

where $p(t, n)$ is molecular weight distribution of the polymer. The zero moment $(p=0)$ is the time-dependent molar concentration of the polymer and the first moment $(p=1)$ is the mass concentration. Therefore, by applying the moment operation on the above population balance equation for random chain scission, the following ordinary differential equation is achieved.

$$
\frac{d p^{(p)}}{d t}=-k p^{(p)}(n)+\underbrace{\frac{1}{n+1} k p^{(p)}}_{\int_{0}^{\infty} n^{p} d n \int_{n}^{\infty} k p(n, t) \underbrace{\left.\frac{1}{n+1} k n^{\prime}\right)}_{\frac{1}{n^{\prime}}}}
$$

Therefore, applying the moment operation on the defined integro-differential equations for 
polymer, polymer radical, and the monomer leads to a set of ordinary differential equations that are easy to solve.

\subsection{PHOTOREACTOR DESIGN}

Reaction system, lamp technology, and chemical engineering are the basis of the photoreactor design and photochemical engineering. The proper combination of these three aspects leads to obtain suitable photoreactor concepts. Nevertheless, most of the commercial photoreactor systems are still based on empirical developments coupled with successive improvements and on the expertise of the manufacturers.

The design of a photoreactor for the application of AOTs is based on the basic principles of chemical reaction engineering. Therefore, the following issues have to be taken into account as the particular concepts of photochemical reaction engineering (Braun, 1993):

- Specific excitation of the auxiliary oxidant or photocatalyst,

- Total absorbance of electromagnetic radiation,

- Minimization of radiation losses by reflection and scattering,

- Perfect mixing within the photoreactor,

- Optimal oxygen saturation in aqueous phase,

- Special consideration of the lamp convergence and reactor geometry and the spatial separation between the lamp and the solution,

- Efficient cooling of the lamp to achieve isothermal condition,

- Precise description of the radiation distribution within the reactor volume, 
- Description of the photoreactor/photochemical system by proper combination of spatially defined energy absorption with the classical mass and momentum balances.

The classical chemical reaction engineering provides mathematical concepts to describe the ideal mass balances and reaction kinetics of commonly used reactor types (Levenspiel, 1972). Among different types of photoreactors, batch recirculation systems are considered as a good choice to degrade the highly contaminated wastewater. A qualitative comparison between the batch and batch recirculation systems for $\mathrm{UV} / \mathrm{H}_{2} \mathrm{O}_{2}$ is presented in Figure 2.7. In both cases, an identical photon flow $p$ of the lamp $(L)$, total absorbance of all incident photons and a sufficient concentration of $\mathrm{H}_{2} \mathrm{O}_{2}$ are assumed. According to Equation (2.70), the molecule $M$ is attacked by hydroxyl radicals yielding the primary oxidation product, $M_{1}^{\text {ox }}$. In the case of complete mineralization, $M_{1}^{\mathrm{ox}}$ is oxidized to $M_{2}^{\mathrm{ox}}$ and finally to $M_{\mathrm{i}}^{\mathrm{ox}}$, and ultimately water and carbon dioxide.

$M+{ }^{\bullet} \mathrm{OH} \rightarrow \mathrm{M}_{1}^{o x}+\cdot \mathrm{OH} \rightarrow \cdots \rightarrow M_{i}^{o x}$

Assuming very fast photo-induced production of ${ }^{\circ} \mathrm{OH}$ and the oxidation reactions, two different concentration profiles could be considered theoretically as shown in Figure 2.7. In the batch system $M$ is attacked by ${ }^{\circ} \mathrm{OH}$ and then it is oxidized to $M_{1}^{\text {ox }}$.

${ }^{\circ} \mathrm{OH}$ radicals are preferably scavenged by $M$ due to the excess amount of $M$ at the beginning of the experiment (Figure 2.7 (a)). Consequently, the concentration of $M_{1}^{\mathrm{ox}}$ increases and competes with $M$ for ${ }^{\circ} \mathrm{OH}$ and leads to a decrease in ${ }^{\circ} \mathrm{OH}$ concentration. Ultimately, the concentration of $M_{\mathrm{i}}^{\mathrm{ox}}$ increases. Hence, the batch photoreactor system is the best choice for the partial oxidation of pollutants in water. As shown in Figure 2.7 (b) a different concentration profile is achieved in the batch recirculation photoreactor. In this system, small fractions of fluid are continuously 
removed from the photoreactor and pumped through the photoreactor. Due to the same absorbed photon, the same amount of ${ }^{\circ} \mathrm{OH}$ is produced in this system as in the batch mode. In this system, based on the residence time of the solution within the photoreactor, a high concentration of $M_{1}^{\text {ox }}$ could be produced. In this case, both $M$ and $M_{1}^{\text {ox }}$ compete for ${ }^{\circ} \mathrm{OH}$ leading to high concentrations of $M_{\mathrm{i}}^{\mathrm{ox}}$. The solution is then returned to the tank and diluted by mixing. Consequently, the batch recirculation system is the best choice for the mineralization of refractory pollutants with the purpose of TOC or DOC reduction. However, the efficiency of the batch recirculation system is highly dependent on the recirculating flow rate. The flow rate should be such that in each pass less than $5 \%$ of mineralization takes place (Oppenländer, 2003).

Different approaches to the photoreactor analysis and design are based on the fact that commercial size photoreactors most likely operate under nonuniform concentrations, temperature, and most importantly radiation light distribution. Therefore, point values of reaction rates are basically not uniform and in many cases are completely different from global ones. In some cases, the problem of nonuniformities in concentration and temperature may become less rigorous by good mixing. However, mixing can never provide the uniform photon distribution if geometric effects (distance from the radiation source to each different point inside the photoreactor) or attenuation effects (due to radiation absorption by the dominant species) have formed a spatial distribution of the absorbed photons. Therefore, in most cases, the nonuniform light distribution is inevitable. In the case of photoreactors, the reactor design needs to include: (1) the radiation source (lamp) specifications (shape and dimensions, output power, and its spectral distribution), (2) the geometrical arrangement of the radiation source relative to the reaction space, and (3) the radiation entrance system into the reactor (mode, construction 
a)

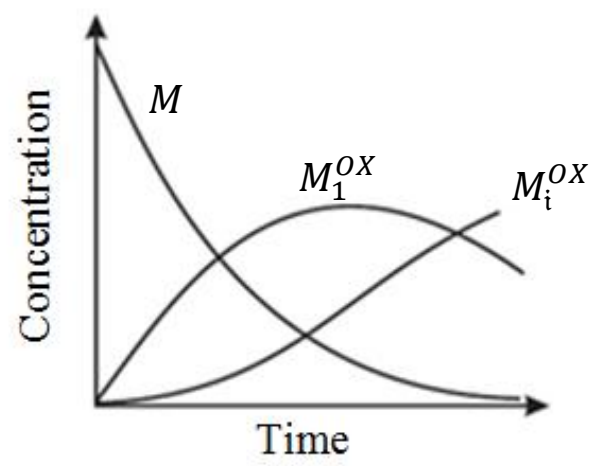

b)

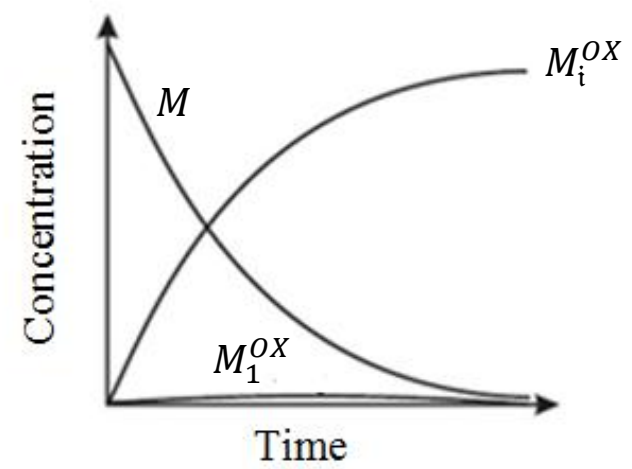

Figure 2.7. Comparison of the concentration-time profiles in a batch (a) and a batch recirculation photoreactor (PR) system (b) (Oppenländer, 2003). 
materials, and cleaning procedure). These specific considerations in photoreactor design are in addition to the general requirements for the reactor design output that must provide such as requirements for raw materials, operating conditions (single or multiphase system), type of hydrodynamic operation (continuous flow, batch, and semibatch) and recycling, the number of reactors and reactor geometry (shape and dimensions), reactor flow rate or reaction time, reactor temperature and pressure, mixing requirement, heating and/or cooling requirements, reactor control safety requirements, and waste disposal specifications and other environmental controls.

\subsection{PHOTOREACTOR MODELING}

Modeling of a photoreactor requires the solution of the radiant energy balance in addition to the momentum, thermal, and mass conservation equations which are needed for the reactor modeling based on the first principles. The radiation balance is generally treated separately from the thermal energy balance because of the negligible thermal effects of the photochemically useful energy. Therefore, one is mainly concerned with the kinetic effects of the employed radiation.

In an isothermal photochemical reactor, mass and radiant energy balances are coupled through the reaction rate terms, and in this case there is no need to have the thermal balance as it is done in isothermal condition. This coupling is due to the effect of the radiation-absorbing-reactant concentration on the radiation absorption by reacting medium. The solution of momentum balance is not coupled with the mass and energy balances. This situation imposes on the photochemical reactor design the same restrictions with regard to an a priori design as in any other type of reactor system. However, photoreactor modeling and especially turbulent and multiphase flows are the areas where the literature lacks sufficient information in fundamental 
equations of general and proven validity for predicting flow behaviour. Therefore, modeling of mass and energy balances under these circumstances has the same flaws in photochemical systems as in the whole area of chemical reaction engineering.

\subsection{PHOTOREACTOR SCALE-UP}

One of the main problems in photochemical reaction engineering is correspondent to the photoreactor scale-up. The traditional and entirely empirical methodology for scaling-up starts from laboratory experiments followed by the gradual increase in size of the reactor up to the desired size. Although this approach seems simple, it needs high investment while scaling-up methodology using mathematical models based on the principle of chemical engineering can decrease the expensive and time-consuming steps. In case of photoreactors, increasing the lamp geometry and the electrical input power results in variation of radiant exitance. Therefore, in order to carry out a practical scaling-up and to optimize a photoreactor, special consideration to the radiant exitance must be taken into account.

There are different approaches in the open literature about the modeling of photoreactors in large scale. Giménez et al., (1999) studied the photocatalytic treatment of phenol and 2, 4dichlorophenol using two different pilot size reactors. Their study involves the combination of fundamental principles together with adjustable constants that depend directly on the reactor shape and configuration. Their results signify that further studies are needed to apply the kinetic model to larger size reactors. Labas et al. (2002) in another approach studied a model for scalingup a homogeneous photoreactor. The model was experimentally validated in pilot-size photoreactor. In their study, the kinetic parameters were estimated in a batch flat-plate 
photoreactor and they were employed in a mathematical, fully deterministic model of a continuous-flow pilot-scale photoreactor without employing any adjustable parameters. Good agreement between model predictions and the experimental data were achieved. Rodriguez et al. (2005) studied the elimination of aromatic compounds present in surface water by photo-Fenton process. They used a solar simulator and a prototype parabolic collector as a laboratory-scale photoreactor to find the parameters of those key factors to be used in the compound parabolic collector pilot plant reactor. They concluded that the constants obtained in both reactors (laboratory-scale and pilot-scale) were practically the same since they depend neither on the radiation source nor on the geometry of the reactor. Thus, laboratory-scale data could be used for pilot-plant scale calculations. Also, Satuf et al. (2007) proposed a methodology to scale-up photocatalytic slurry reactors using a laboratory-scale information for the degradation of 4chlorophenol and applying the obtained kinetic expressions to a reactor of different geometry and operation mode. The simulation results were in good accordance to the experimental data in the large-scale photoreactor. Finally, Marugan et al. (2009) proposed a method for the scaling-up of slurry reactors for the photocatalytic oxidation of cyanide. The procedure was based on the determination of the intrinsic kinetics of the reaction at the laboratory scale to describe the explicit dependence of the reaction rate with the radiation profiles existing in the photoreactor. Based on this information, the model of a larger scale reactor has predicted a good accuracy with its experimental performance. However, further investigation is needed to achieve a methodology that can be used for scaling-up photoreactors from laboratory-scale to commercial size without employing any adjustable parameters for each application of the same process. 


\subsection{SYNTHETIC WATER-SOLUBLE POLYMERS AS MODEL}

COMPOUNDS IN AOTs

As mentioned earlier, environmental pollution by this type of widely used polymers in wastewater has been considered a major concern. Generally, four end results are considered in exposure of polymers in environment (Swift, 1993):

- They may partially degrade by nonbiologically mediated processes.

- They may be biodegradable or degrade partially by biologically mediated processes.

- They may mineralize to gaseous products, water, salts, and biomass.

- They may remain in the environment without significant change.

Although some of the synthetic water-soluble polymers may be partially biodegradable (e.g. polyvinyl alcohol), microorganisms that can degrade them are not ubiquitous within the environment. On the other hand, the biodegradation may be incomplete and produce some other nonbiodegradable products such as olefins. Therefore, without continued biodegradation, the fragments and byproducts remain in the environment. Their fate in the environment could be recalcitrance without harmful side effects, toxicity to animals and soil microbes, depletion of soil fertility, and carrying heavy metals through the environment. This uncertain fate evidently poses a huge problem that needs to be addressed. On the other hand, due to their water solubility, these types of polymers have received little attention in public media than the packaging plastics that are visibly discarded in the environment. In many industrial facilities that deal with these polymers, they can be present as contaminants in wastewaters, either in discarded process water or as a result of washing equipment or containers. The most widely used industrial water-soluble polymers of potential environmental concern are listed in Table 2.2. 
Table 2.2. Synthetic water-soluble polymers of potential environmental concern.

\begin{tabular}{lc}
\hline \multicolumn{1}{c}{ Polymer Type } & Acronym \\
\hline Poly (acrylic acid) & PAA \\
Poly (methacrylic acid) & PMA \\
Poly (ethylene oxide) & PEO \\
Poly (acrylamide) & PAM \\
Poly (vinyl alcohol) & PVA \\
Poly (vinylpyrrolidine) & PVP \\
\hline
\end{tabular}

PAA and PMA derivatives due to their capability to absorb large quantities of water are applied as superabsorbers (for example in production of baby diapers), in pharmacy, dentistry and in the paints industry as thickening agents, diluents, and removers (Williams et al., 2007; Kaczmarek et al., 1998). PEO is widely used in the production of surfactants, explosives, cosmetics, lubricants and in heat transfer fluids (Giroto et al., 2010). PAM is also a versatile chemical, used as an additive to modify surface and viscosity properties of a number of important industrial substances (Giroto et al., 2008). PVA is used in various industries such as textiles, printing, paper-coating, pharmaceuticals, and detergent-based industries (Hsu et al., 2011). PVP is used in cosmetics (hairsprays), textile, pharmaceutics, or as an impermeability agent in building materials such as cement, wood, and sealing composites (Hassouna et al., 2011). Among most widely used water-soluble polymers, PEO and PAA have the simplest molecular structure and represent the appropriate model compounds to study the degradation characteristics of polymers. 
Studies on the degradation of the synthetic water-soluble polymers by AOTs are not many in the open literature. Most studies have centered on the thermal degradation of polymers (Lépine, and Gilbert, 2002; Vijayalakshmi et al., 2006; Fernández and Fernández, 2008; Vrandečić, et al., 2010) and determining the monomer yield and the rate of change of average molecular weight ( Kanwal and Pethrick, 2004; Aarthi et al., 2007; Daraboina and Madras, 2009; Hassouna et al., 2009; Shukla et al., 2009; Hassouna et al., 2011). However, the information on the degradation of the synthetic water-soluble polymers using different AOTs specially based on surrogate parameters such as chemical oxidation demand (COD), dissolved organic carbon (DOC), and total organic carbon (TOC) is scarce in literature (Giroto et al., 2008; Giroto et al., 2010; Lu et al., 2012). The TOC is the most applicable parameter for the global determination of organic matter in water and wastewater (Oppenländer, 2003).

\subsection{RESEARCH OBJECTIVES}

Based on the extensive literature review, it is found that there is little information on the following issues:

a. Polymer degradation by different AOTs; most studies are on thermal degradation of polymers;

b. Modeling and statistical analysis of experimental results using different methods of response surface methodology;

c. Mechanistic kinetic modeling of photooxidative degradation of polymers by AOTs because of the complexity of degradation kinetics of high molecular weight compounds; 
d. Computational fluid dynamics (CFD) modeling of AOTs to achieve spatial distribution of different parameters;

e. Investigating the photooxidative degradation of polymers using cascades of photoreactor modules (in series or parallel mode);

f. Considering 3-dimensional local volumetric rate of energy absorption (LVREA), radiation emission, and radiation absorption models in photoreactor modeling and design; and

g. Achieving a methodology for scaling-up a photoreactor directly from laboratory scale to a pilot scale.

Consequently, the main objectives of this thesis are as follows:

a. To investigate the effectiveness of $\mathrm{UV} / \mathrm{H}_{2} \mathrm{O}_{2}$ in a laboratory scale photoreactor using response surface methodology (Central Composite Design) combined with quadratic programming for experimental design, statistical analysis, and optimization of the process;

b. To develop and validate a detailed mathematical model using continuous distribution kinetics to predict the TOC removal by AOTs with time as output variable;

c. To find the intrinsic kinetic constants of the mechanistic kinetic model through an optimization algorithm;

d. To study CFD modeling incorporating the momentum balance, proposed kinetic model, mass balance, and 3-dimensional local volumetric rate of energy absorption;

e. To study the efficiency of the photo-Fenton-like process using response surface methodology (Box-Behnken Design) combined with quadratic programming for experimental design, statistical analysis, and optimization of the process; 
f. To develop and validate a detailed mechanistic kinetic model to predict the TOC removal of the photo-Fenton-like process;

g. Conducting sensitivity analysis to determine the most influential reaction rate constants in the kinetic model for the photo-Fenton-like process;

h. To investigate CFD modeling of the photo-Fenton-like process incorporating rigorous kinetic rate expressions and governing transport equations;

i. To study the effectiveness of photo-Fenton-like process in a batch recirculation system using two photoreactors in series;

j. To develop a methodology for scaling-up the laboratory-scale photoreactor to a pilotscale photoreactor (with different configuration and mode of operation) for polymer photodegradation which requires the solution of momentum, multicomponent mass conservation, and also 3-dimensional radiation balances along with two submodels for radiation emission and radiation absorption models; and

k. To validate the scaling-up methodology in the pilot-scale photoreactor using CFD modeling; 


\section{Chapter 3}

\section{MATERIALS AND METHODS}

\subsection{MATERIALS}

The materials used in the experiments are listed as follows:

- Poly(ethylene oxide) with an average molecular weight $\left(\mathrm{M}_{\mathrm{w}}\right)$ of $2 \times 10^{5} \mathrm{~g} \mathrm{~mol}^{-1}$ (VWR Canada)

- Poly(acrylic acid) $35 \% \mathrm{wt}$ with an average molecular weight $\left(\mathrm{M}_{\mathrm{w}}\right)$ of $10^{5} \mathrm{~g} \mathrm{~mol}^{-1}$ (VWR Canada)

- Poly(vinyl alcohol) with an average molecular weight $\left(\mathrm{M}_{\mathrm{w}}\right)$ of $13 \times 10^{4} \mathrm{~g} \mathrm{~mol}^{-1}$ (VWR Canada)

- Hydrogen peroxide 30\%wt (VWR Canada)

- Ferric chloride hexahydrate (Sigma-Aldrich)

- Sulfuric acid 1N (EMD Chemicals)

- Sodium hydroxide 1N (EMD Chemicals)

- $\quad$ Phosphoric acid 20\% (VWR Canada)

- Distilled water

\subsection{EXPERIMENTAL SETUPS}

In this section, two experimental setups including a laboratory-scale batch recirculating system and a pilot-scale annular photoreactor to validate the results of scaling-up methodology are presented. 


\subsubsection{Laboratory-Scale Photoreactor}

As mentioned in Section 2.3, batch recirculation systems with small conversion per pass (less than 5\%) are considered as a good choice to study kinetics of the degradation of the highly contaminated wastewater with the purpose of TOC diminution. Therefore, in this study a laboratory-scale batch recirculation photoreactor set up (Figure 3.1) including two photoreactors in series (could be used either as a single photoreactor or two photoreactors) was employed. Each of the photoreactors can be used individually in a batch recirculation mode. The system can be also used in continuous mode. The description of the laboratory-scale photoreactor is presented in Table 3.1. The reactor configuration enables a simple mathematical representation for both mass and radiation balances. A small annular photoreactor (SL-LAB, Siemens Inc.) with the annular space of $1.33 \mathrm{~cm}$ was used as a part of the recycle system including a centrifugal magnetic pump (Model RK-72012-10, Cole-Parmer), an all glass heat exchanger for controlling the temperature, and a large volume tank with provisions for sampling and temperature measurements. The system was also equipped with a by-pass valve to control the flow rate and to provide a relief to the pump pressure. The low-pressure Hg lamp at $14 \mathrm{~W}$ with $\mathrm{UV}$ emission peaks at $254 \mathrm{~nm}$ (LP4130, Siemens Inc.), sealed with the quartz sleeve was positioned at the centerline of the photoreactor with stainless steel housing. This particular geometry (very small annular space) and the method of irradiation lead to have a good approximation of an isoactinic condition (uniform light distribution) in the photoreactor (Marugan et al., 2009).

\subsubsection{Pilot-Scale Photoreactor}

A pilot-scale annular photoreactor (Figure 3.2) is used in order to validate the scaling-up methodology. The general methodology for scaling-up for all applications is the same. One must precisely model the laboratory-scale photoreactor with almost the same tools that are used in the 
larger scale. Therefore, both laboratory-scale and pilot-scale photoreactor must be strictly using the same wavelength spectral distribution of the employed input power. The description of the pilot-scale photoreactor is presented in Table 3.2. The pilot-scale system could be employed in either batch or continuous mode. A single ended UV lamp (Ushio America, Inc.) sealed with the quartz sleeve was inserted in the center of the photoreactor.

\subsection{ANALYTICAL TECHNIQUES}

\subsection{1. pH Measurement}

The $\mathrm{pH}$ was measured by a potentiometric $\mathrm{pH}$ meter (model 230A+ from Thermo Orion) using a glass indicator electrode along with a reference electrode. The indicator and reference electrodes were combined in one. Two buffers of $\mathrm{pH} 4$ and 7 were used to calibrate the $\mathrm{pH}$ meter before measurements. These two buffers were chosen in the expected sample ranges. During $\mathrm{pH}$ measurements, the temperature was displayed automatically and recorded. The calibration was verified before using.

\subsubsection{TOC Analyzer}

The TOC was measured by a Tekmar Dohrmann's Apollo 9000 TOC/TN analyzer. The analyzer is a combustion type $\left(680\right.$ to $\left.1000^{\circ} \mathrm{C}\right)$ with a patented reusable platinum catalyst for the lowest detection limit while maximizing TOC recovery. The Non-Dispersive Infra-Red (NDIR) detector

in the Apollo 9000 TOC Analyzer is sensitive to very low levels of TOC $\left(4 \mathrm{mgC} \mathrm{L}^{-1}\right)$ which directly and specifically measures the carbon dioxide generated by the oxidation of the organic carbon in the sample. 


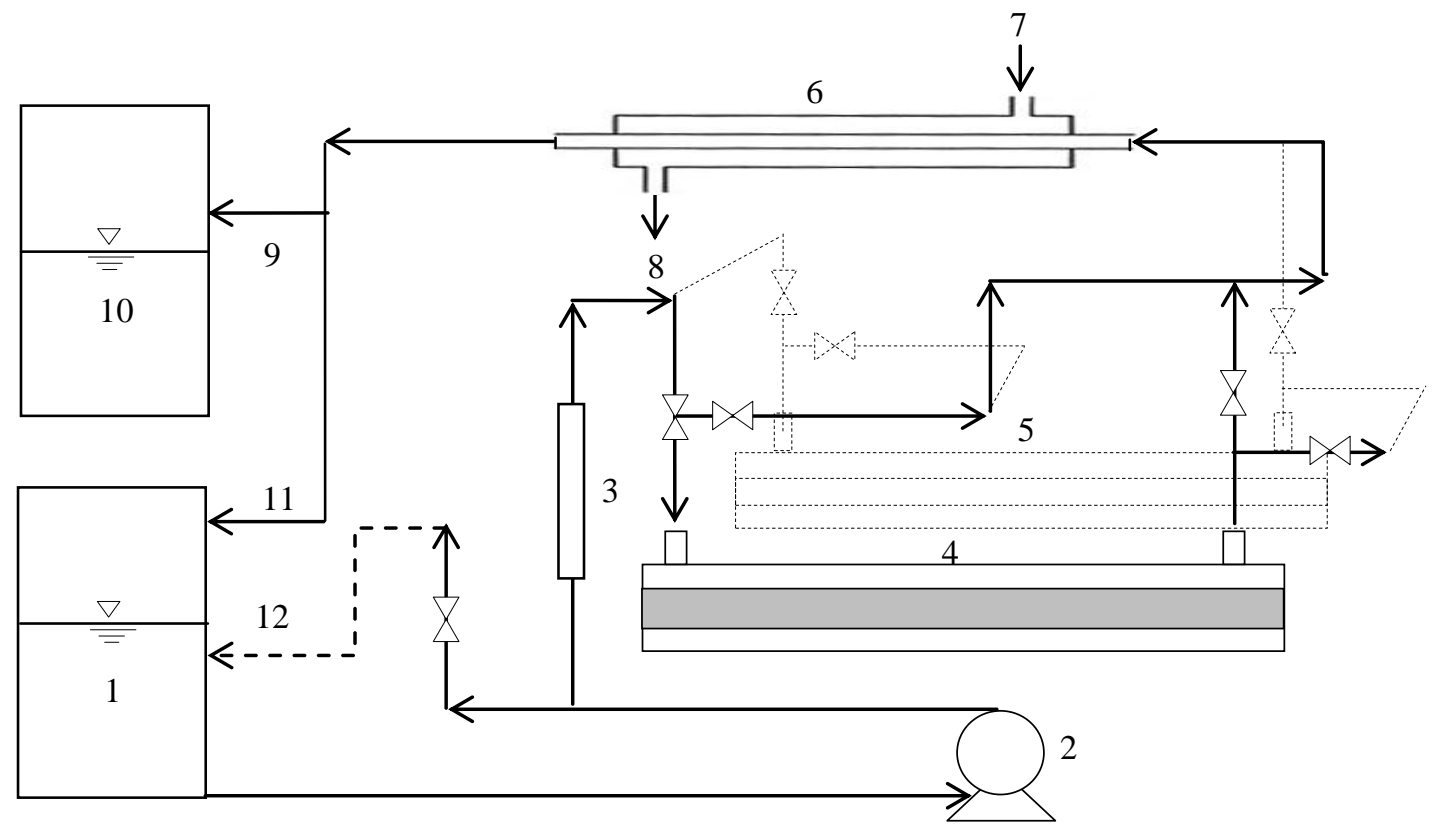

Figure 3.1. Schematic diagram of the laboratory-scale batch recirculating system.

(1) Reservoir tank, (2) Pump, (3) Flow meter, (4) UV-C photoreactor, (5) UV-C photoreactor (6) Heat exchanger, (7) Cooling water in, (8) Water out, (9) Continueous mode (10) Collection tank (11) Batch recirculation mode, and (12) Bypass. 
Table 3.1. Description of laboratory-scale photoreactor.

\begin{tabular}{llc}
\hline Item & & Specification \\
\hline Photoreactor & Type & Batch Recirculation \\
& Inner Diameter & $2.44 \mathrm{~cm}$ \\
& Outer Diameter & $5.11 \mathrm{~cm}$ \\
& Length & $30.5 \mathrm{~cm}$ \\
& Volume & $0.46 \mathrm{~L}$ \\
Tank Volume & & $8 \mathrm{~L}$ \\
Operating Flow Rate & & $0.1-10 \mathrm{~L} \mathrm{~min}{ }^{-1}$ \\
Lamp & Type & LP Hg UV \\
& Wavelength & $254 \mathrm{~nm}$ \\
& Length & $20.5 \mathrm{~cm}$ \\
Temperature & Output power & $4 \mathrm{~W}$ \\
Pressure & & $22^{\circ} \mathrm{C}$ \\
\hline
\end{tabular}




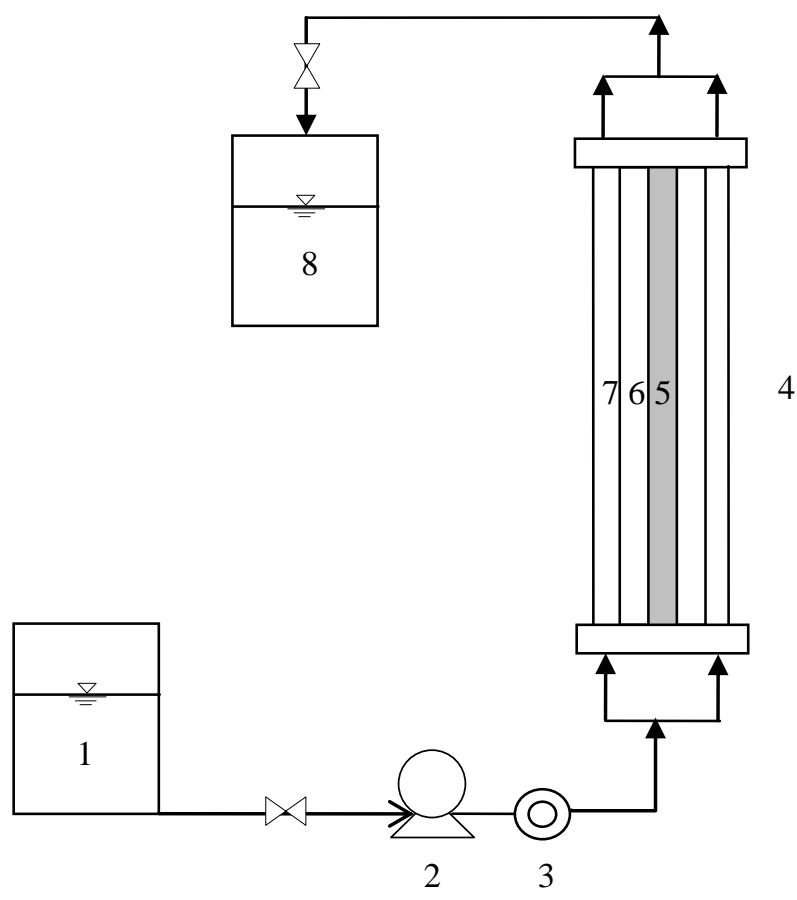

Figure 3.2. Schematic diagram of the pilot-scale flow through system.

(1) Feed tank, (2) Pump, (3) Flow meter, (4) Photoreactor, (5) UV-C lamp, (6) Quartz sleeve, (7) Annular space, (8) Collection tank 
Table 3.2. Description of pilot-scale photoreactor.

\begin{tabular}{llc}
\hline Item & & Specification \\
\hline Photoreactor & Type & Annular continuous flow \\
& Inner Diameter & $1.55 \mathrm{~cm}$ \\
& Outer Diameter & $9.72 \mathrm{~cm}$ \\
& Length & $90 \mathrm{~cm}$ \\
& Volume & $7 \mathrm{~L}$ \\
Operating Flow Rate & & $0.1-0.5 \mathrm{~L} \mathrm{~min}{ }^{-1}$ \\
Lamp & Type & $\mathrm{LP} \mathrm{Hg} \mathrm{UV}$ \\
& Wavelength & $254 \mathrm{~nm}$ \\
& Length & $84 \mathrm{~cm}$ \\
Temperature & Output power & $13 \mathrm{~W}$ \\
Pressure & & $22^{\circ} \mathrm{C}$ \\
\hline
\end{tabular}


The analyzer is able to measure TOC concentrations within the range of $4-25,000 \mathrm{mgC} \mathrm{L}^{-1}$. Approximately, $15-40 \mathrm{~mL}$ sample was filled in a sampling vial and was placed in the auto sampler. Through running TOC Talk Software (Version 3.5), the TOC standard calibration analysis was carried out using the working standard solutions (50, 1000, 2000, $3000 \mathrm{ppm})$. Measurement of each sample was repeated in 3 times and an average value was reported as the TOC reading. A response factor of the instrument correlates the raw counts to a known amount of organic carbon in the standard. The calibration of the TOC curve was carried out every 6 months during the experimental study period. During that period several standard samples were used for quality control. The calibration curves are provided in appendix A.

The steps during the TOC analysis (Apollo 9000 TOC Analyzer Operation Manual, 2009) are described as follows:

1. Sampling: Samples were injected into the analyzer with the help of an automated syringe from the sampling bottle. The sample injection valve automatically selects the appropriate sample volume for the optimum measuring range.

2. Inorganic Carbon (IC) detection: $20 \%$ phosphoric acid was added to lower the pH so that inorganic carbon was sparged off as $\mathrm{CO}_{2}$. This was measured to get IC content and to ensure that it was not carried over into the TOC.

3. Oxidation: The high temperature combustion method was used to achieve the total and complete oxidation of the samples, i.e. organic carbon to $\mathrm{CO}_{2}$ in the combustion chamber.

4. TOC measurement: Carrier gas (purified air) sweeps sample gas containing $\mathrm{CO}_{2}$ and water vapour out of the combustion furnace. The sample then travels through tubing, cooled by a fan to condense water vapour and finally to trap water where water was collected. Then, the gas sample 
passes through a semi-permeable Nafion ${ }^{\circledR}$ tube to further remove any moisture. This moisture free sample gas was then passed through the corrosive scrubber to remove any halides and finally to the NDIR-detector. Here, the $\mathrm{CO}_{2}$ was measured and the result was displayed as total organic carbon (TOC) $\left(\mathrm{mgC} \mathrm{L}^{-1}\right)$.

5. Cleaning: Before introducing a sample, Apollo 9000 automatically rinses the syringe to eliminate any contaminant that may interfere with the testing process. This rinsing occurs through a loop sequence where the syringe is filled and discarded with distilled water.

\subsubsection{UV Spectrometer}

The light absorption by the polymer solution to find the extinction coefficient was measured using a UV-vis spectrophotometer (Ultrospec 1100 pro, 66Amersham Biosciences, USA) at ambient temperature $\left(25 \pm 5^{\circ} \mathrm{C}\right)$. of the A polymer solution of $3 \mathrm{~mL}$ was analyzed in a UV-vis spectrophotometer at wavelength of $250-900 \mathrm{~nm}$. The spectrophotometer has the ability to measure the absorbance, percent transmission, and concentration values. It measures the absorbance of samples based on the amount of light passed through a sample relative to a blank. While percent transmission mode measures the amount of light that has passed through a sample relative to a blank, it displays the result as a percentage. The concentration mode is used when a conversion factor is known, and it is required to convert the absorbance measurement for a sample at a specific wavelength into a concentration. 


\subsection{ERROR ANALYSIS}

For the TOC analyzer, at least two samples in three replicates were measured in each experimental run. Also, the background TOC of at least two distilled water samples, as the blank samples, was measured before measuring each sample. The standard deviation was calculated for each reading. Low value of standard deviation $(<0.3)$ indicates the good reproducibility and repeatability of the data measurements. Also, the TOC analyzer was calibrated every six months based on the supplier guidelines. The calibration was verified by placing standard samples between the trials. For the spectrometric analysis, the UV-vis spectrophotometer was autocalibrated everyday and three samples were tested in each measurement.

\subsection{MODELING, STATISTICAL ANALYSIS, AND OPTIMIZATION}

In this section, the experimental design for different AOTs processes including modeling, statistical analysis, and optimization using different methods of response surface methodology are discussed. Also, the computational fluid dynamics package to find the spatial distribution of different parameters is introduced.

\subsubsection{Response Surface Methodology}

Response surface methodology (RSM) is a combination of mathematical and statistical techniques that is an effective tool for developing, improving, and optimizing different processes. In addition to the significant applications in the design, development, and formulation of new products, it could be used in the improvement of existing product designs. The most important application of RSM is in industrial processes, especially where different input 
variables or factors influence some performance measure or quality characteristic of the process. This performance measure or quality characteristic of the process is called response. In many of the real applications of RSM, there is more than one response. The input variable or the factors are called independent variables.

Generally, an experimenter is concerned with a process including a response function $y$ that depends on the controllable input variables $\xi_{1}, \xi_{2}, \ldots, \xi_{\mathrm{k}}$ as follows (Myers et al., 2009).

$y=f\left(\xi_{1}, \xi_{2}, \ldots, \xi_{k}\right)+\varepsilon$

where $\varepsilon$ is a term that represents other sources of variability which is not considered in $f$. Therefore, $\varepsilon$ (statistical error) includes effects such as measurement error on the response, other sources of variation that are inherent in the process or system such as background noise, and the effect of other variables. In most of the RSMs, it is more convenient to transform the natural variables to $X_{1}, X_{2}, \ldots, X_{\mathrm{k}}$ which are usually defined to be dimensionless with mean zero and the same spread or standard deviation. The response function in terms of the coded variables is given as follows.

$y=f\left(X_{1}, X_{2}, \ldots, X_{k}\right)+\varepsilon$

Since the true form of the response function is not known, it can be approximated. In fact, a suitable use of RSM is highly dependent on the experimenter's ability to develop an appropriate approximation of $f$. In small region of the independent variables, the low order (first-order or second-order model) is usually appropriate. The first-order model for the two independent variables considering the interaction among the variables is presented as follows:

$y=\beta_{0}+\beta_{1} X_{1}+\beta_{2} X_{2}+\beta_{12} X_{1} X_{2}$ 
where $\beta_{0}$ is the constant coefficient (intercept term), $\beta_{1}$ and $\beta_{2}$ are the linear coefficients, and $\beta_{12}$ is an interaction coefficient. The interaction term introduces the curvature into the response function. However, in many cases the curvature in the true response function is such strong that even adding the interaction term to the first-order model is not adequate. Therefore, the firstorder model has to be upgraded by adding higher order terms. The second-order model for the case of two variables can be presented by the following equation:

$y=\beta_{0}+\beta_{1} X_{1}+\beta_{2} X_{2}+\beta_{11} X_{1}^{2}+\beta_{22} X_{2}^{2}+\beta_{12} X_{1} X_{2}$

where $\beta_{11}$ and $\beta_{22}$ are quadratic coefficients. This model would give a good approximation to the response surface where there is substantial curvature in the true response function $f$. The second order model is widely used in the RSM because of the following reasons:

- The model is very flexible to take on a variety of functional forms. Hence, it could be considered as an approximation to the true response surface.

- The parameter estimation (the $\beta$ 's) in the second order model could be easily carried out using the method of least squares.

- There are numerous practical experiences showing the reliability of the second order model in solving real response surface problems.

The general form of the second-order model is given by the following equation:

$Y=\beta_{0}+\sum_{j=1}^{k} \beta_{j} X_{j}+\sum_{j=1}^{k} \beta_{j j} X_{j}^{2}+\sum_{i=1}^{k-1} \sum_{j=2}^{k} \beta_{i j} X_{i} X_{j}+e$

where $Y, \beta_{0}, \beta_{i}, \beta_{i i}$, and $\beta_{i j}$ are the predicted response, the constant coefficient (intercept term), the linear coefficients, the quadratic coefficients, and the interaction coefficients, respectively. 
The parameters $X_{i}$ and $X_{\mathrm{j}}$ are independent variables, where $k$ and $e$ are the number of factors and the residual term allowing uncertainties between observed and predicted values, respectively. Generally, RSM is used in many industrial problems in the following categories:

- Mapping a response surface over a particular region of interest. The approximation of the true response function over the region around the current operating conditions helps the engineers to predict the changes in the yield of the process by any readjustment to the operating conditions.

- Optimization of the response: In industrial applications, the determination of the operating conditions to optimize the process response is of special significance.

- Selection of operating conditions to achieve specifications or customer requirements: In many industrial applications, there is more than one response function that needs to be optimized. The superimposing proper response surface contours is often used to solve these complex optimization problems.

There are several steps in the building of empirical models using RSM that are discussed as follows:

\section{Development of linear regression models}

The practical application of RSM requires the development of an approximation of a model for the true response surface. The underlying true response surface is typically driven by some unknown physical mechanism. The approximating model is based on observed data from the process or system which is an empirical model. Multiple regression is a collection of statistical techniques which are used to build the types of empirical models required in RSM. As mentioned earlier, the second-order model is widely used in most of the industrial applications. 


\section{Estimation of the parameters in linear regression models}

The estimation of parameters in linear regression models is usually carried out by the least squares method. The method of least squares chooses the $\beta$ 's in the linear regression models in Equation (3.5) such that the sum of squares of errors $(e)$ are minimized.

\section{Hypothesis testing in multiple regression}

In multiple linear regression problems, certain tests of hypotheses about the model parameters are useful in measuring the effectiveness of the model. The most widely used tests are the Fischer's $F$-value and $p$-value tests. Generally, Fischer's $F$-value is used to determine the significance of the regression coefficients of the variables while $p$-value determeines the significance of the variable that may indicate the pattern of interaction among variables. $F$-value could be determined by the following equation (Myers et al., 2009):

$F=\frac{M S_{M}}{M S_{E}}$

where $M S_{M}$ is the mean sum of squares due to the model and $M S_{E}$ is the mean sum of squares due to error (residuals). $M S_{M}$ and $M S_{E}$ could also be found by the following equations:

$M S_{M}=\frac{S S_{M}}{d_{M}}$

$M S_{E}=\frac{S S_{E}}{d_{E}}$

where $S S_{M}, S S_{E}, d_{M}$, and $d_{E}$ are sum of squares of the model, sum of squares of the residuals (error), degree of freedom for the model, and degree of freedom of the residuals, respectively. The $p$-values are associated with $F$-values as they are useful to show whether $F$-values are large 
enough to indicate the statistical significance. The significance of the regression could be also verified through the coefficient of multiple determination $R^{2}$ which is defined as follows:

$R^{2}=\frac{S S_{M}}{S S_{T}}=1-\frac{S S_{E}}{S S_{T}}$

where total sum of squares $\left(S S_{T}\right)$ is given by the following equation:

$S S_{T}=S S_{E}+S S_{R}$

$R^{2}$ is a measure of the amount of the reduction in the variability of $y$ obtained by using the regressor variables $X_{1}, X_{2}, \ldots, X_{\mathrm{k}}$ in the model. However, a large value of $R^{2}$ does not necessarily indicates a good regression model. Adding a variable to the model always increases $R^{2}$, regardless of whether the additional variable is statistically significant or not. Thus, it is possible for models that have large values of $R^{2}$ to yield poor predictions of new estimates of the mean response. Therefore, some regression model builders prefer to use an adjusted $R^{2}$ statistic that is given by the following equation (Myers et al., 2009):

$R_{a d j}^{2}=1-\frac{S S_{E} /(n-p)}{S S_{T} /(n-1)}=1-\frac{n-1}{n-p}\left(1-R^{2}\right)$

where $n$ and $p$ are the number of independent variables and the coefficients, respectively. $R_{a d j}^{2}$, unlike $R^{2}$, is not always increased as variables are added to the model. In fact, if unnecessary terms are added, the value of $R_{a d j}^{2}$ is often decreased. Therefore, high values of $R^{2}$ and $R_{a d j}^{2}$ that are very close to each other imply the significance of the regression.

\section{Validation of model adequacy}

Generally, in RSM, it is always essential to: (i) validate the fitted model to ensure that it provides an adequate approximation to the true system, and (ii) verify that none of the least square 
regression assumptions are violated. Proceeding with exploration and optimization of a fitted response surface likely gives misleading results unless the model provides a satisfactory fit. Therefore, several techniques for validating model adequacy are presented in this section.

The residual from the least square fit (difference between the model prediction and the observation) is a good indicator for the model adequacy. A validate of the normality assumption may be made by constructing a normal probability plot of the residuals. If the residual plot lies on the straight line, then the normality assumption is satisfied. Another plot to validate the model adequacy is the plot of residuals over the predicted response. If the residuals scatter randomly on the display, the model is adequate (Mehrvar et al., 2000).

The scaled residuals are often more preferable as they convey more information than the ordinary least square residuals. One of the scaled residuals is studentized residual. The studentized residuals are found by dividing the residuals by their standard deviations. If the points scatter randomly between the outlier detection limit of -3 and +3 , the model adequacy is satisfied.

\subsubsection{Central composite design}

Central composite design (CCD) is the most popular second-order design in use by practitioners. However, some important characteristics have to be taken into account before choosing a response surface design as follows:

- Lead to the good accordance of the model with the observed data,

- Provide an estimate of "pure" experimental error,

- Be robust to errors in control of design levels, and 
- Be cost-effective.

As mentioned earlier, the CCD is without a doubt the most popular class of second-order designs introduced by Box and Wilson in 1951 (Box and Wilson, 1951). It involves $F$ factorial points, $2 k$ axial points, and $n_{c}$ center runs. The factorial points represent a variance-optimal design for a first-order model or a first-order plus two-factor interaction model. Center runs clearly provide information about the existence of a curvature in the system. If the curvature is found in the system, the addition of axial points allows for efficient estimation of the pure quadratic terms. In fact, the three components of the design ( $F$ factorial points, $2 k$ axial points, and $n_{\mathrm{c}}$ center runs) play significant and somewhat different roles as follows:

- Two level factorial or fraction contributes substantially to the estimation of linear terms and two-factor interactions. The factorial points are the only points that contribute to the estimation of the interaction terms.

- The axial points contribute in a large way to the estimation of quadratic terms. Without the axial points, only the sum of the quadratic terms can be estimated. The axial points do not contribute to the estimation of interaction terms.

- The center runs provide an internal estimate of error (pure error) and contribute toward the estimation of quadratic terms.

The areas of flexibility in the use of the central composite design reside in the selection of $\alpha$, the axial distance, and $n_{c}$, the number of center runs. The choice of these two parameters can be very important. The choice of $\alpha$ depends on a great extent on the region of operability and the region of interest. Figure 3.3 and Figure 3.4 show the CCD for $k=2$ and $k=3$, respectively. For the $k=$ 2 case, the value of $\alpha$, the axial distance is $\sqrt{2}$. For $k=3$, the value of $\alpha$ is $\sqrt{3}$. Note that for $k=3$, 
the axial points come through the six faces at a distance from the origin. For $k=2$, the design represents eight points equally spaced on a circle, plus the center runs. For $k=3$ the design represents 14 points all on a common sphere, plus center runs. The value of the axial distance generally varies from 1.0 to $\sqrt{k}$, the former placing all axial points on the face of the cube, the latter resulting in all points being placed on a common sphere. In some cases, two or more center runs are needed while in some other cases one or two runs are sufficient.

\subsubsection{Box-Behnken design}

A creative methodology for three-level designs to fit second-order response surfaces were developed by Box and Behnken in 1960 (Box and Menken, 1960). Box-Behnken design (BBD) is based on the construction of balanced incomplete block designs. For example, a balanced incomplete block design with three treatments and three blocks is presented in Table 3.3.

Table 3.3. A balanced incomplete block design

\begin{tabular}{lccc}
\hline & \multicolumn{3}{c}{ Treatment } \\
& 1 & 2 & 3 \\
\cline { 2 - 4 } Block 1 & $\mathrm{X}$ & $\mathrm{X}$ & \\
Block 2 & $\mathrm{X}$ & & $\mathrm{X}$ \\
Block 3 & & $\mathrm{X}$ & $\mathrm{X}$ \\
\hline
\end{tabular}

The pairing together for treatments 1 and 2 indicates that the design variables $X_{1}$ and $X_{2}$ are paired together in a $2^{2}$ factorial (scaling \pm 1 ) while $X_{3}$ remains fixed at the center $\left(X_{3}=0\right)$. The same applies for blocks 2 and 3, with a $2^{2}$ factorial being represented by each pair of treatments while the third factor remains fixed at 0 . The $\mathrm{BBD}$ is rather comparable in number of design points to the $\mathrm{CCD}$ for $k=3$ and $k=4$; where there is no $\mathrm{BBD}$ for $k=2$. For $k=3$, the $\mathrm{CCD}$ contains $14+n_{c}$ runs while the BBD contains $12+n_{\mathrm{c}}$ runs. 


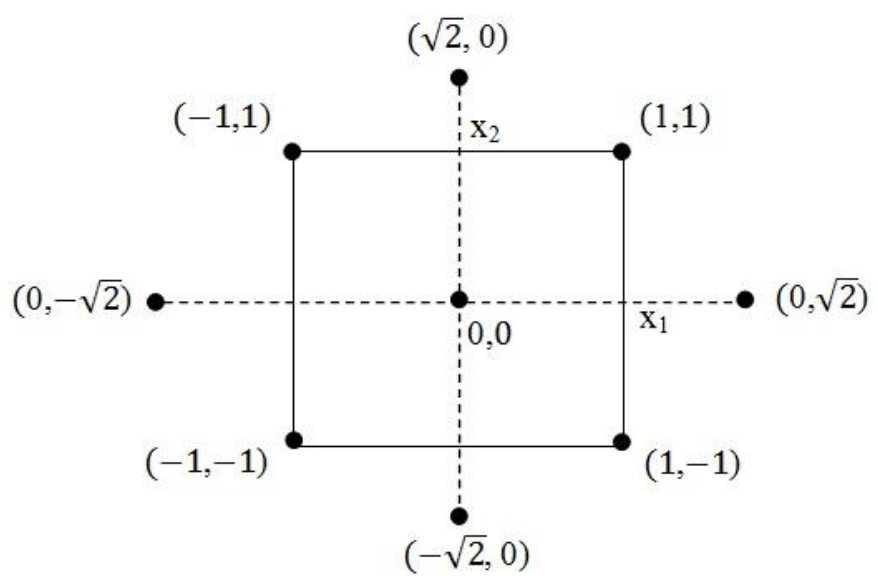

Figure 3.3. Central composite design for $k=2$ and $\boldsymbol{\alpha}=\sqrt{\mathbf{2}}$.
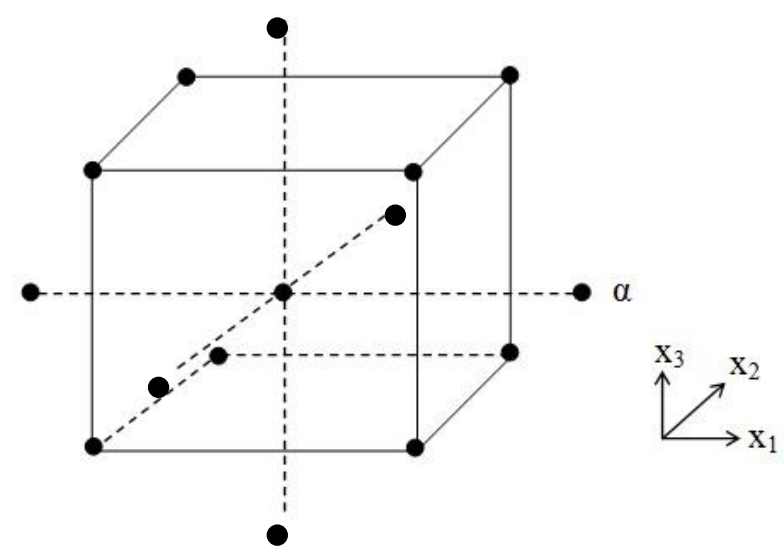

Figure 3.4. Central composite design for $k=3$ and $\boldsymbol{\alpha}=\sqrt{\mathbf{3}}$. 
For $k=4$, the $\mathrm{CCD}$ and $\mathrm{BBD}$ both contain $24+n_{c}$ design points. In many RSM studies, researchers are inclined to require three evenly spaced levels. Thus, the Box-Behnken design is an efficient option and indeed an important alternative to the central composite design. The BoxBehnken design does not substantially deviate from rotatability, and in fact, it is known as a rotatable quadratic design having no fractional factorial points (Zhang et al., 2011). Another important characteristic of the BBD is that it is a spherical design and the variable combinations are at the center and the midpoints of the edges of the variable space. There are no factorial points or face points as shown in Figure 3.5 for the BBD of $k=3$.

The BBD involves all edge points, but the entire cube is not covered. In fact, there are no points

on the corner of the cube or even a distance $\sqrt{2}$ from the design center. However, the use of the BBD should be limited to situations in which one is not interested in predicting the response at the corners of the cube. The spherical nature of the BBD, combined with the fact that the designs are rotatable or near-rotatable, suggests that ample center runs should be used.

\subsubsection{Computational Fluid Dynamics}

While numerous studies have been conducted in modeling AOTs, there are only limited studies on CFD modeling for fundamental understanding of the complex photochemical processes (Alpert et al., 2010; Mohajerani et al., 2010; Santro et al., 2010; Vincent et al., 2011; Duran et al., 2011). The CFD, as a numerical technique, solves the fluid dynamic equations through space and time including the conservations of mass, momentum, and energy (photon balance). These governing equations combined with proper boundary and initial conditions can illustrate physical and chemical changes within a reactor. 


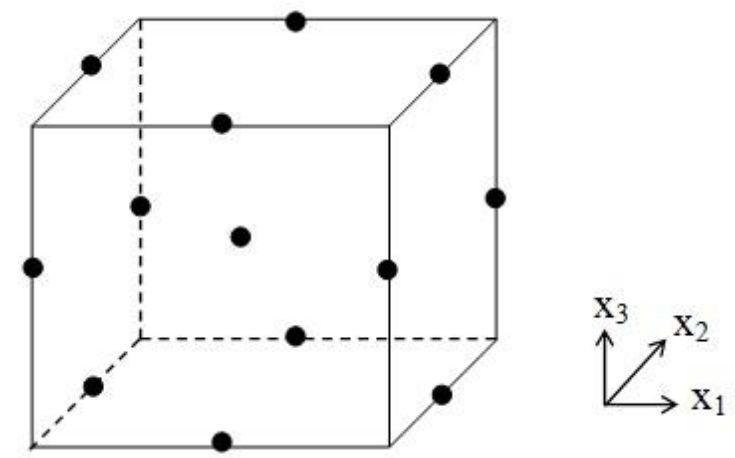

Figure 3.5. Box-Behnken design for $k=3$. 
To simulate the 3D fluid field within the laboratory-scale and pilot-scale photoreactors, COMSOL Multiphysics (Version 3.5) was utilized. COMSOL Multiphysics is a powerful interactive environment for modeling and solving all kinds of Chemical Engineering problems based on partial differential equations (PDEs). With this software, one can easily extend conventional models for one type of physics into multiphysics models that solve coupled physics phenomena - and do so simultaneously. This commercial package is based on the finite element method used for CFD modeling. Finite element method is a numerical approach to find approximate solution to boundary value problems by minimizing an error function and producing a stable solution. In this method, by dividing a structure to several discrete elements and reconnecting the elements at nodes, a set of simultaneous algebraic equations are achieved. In fact, the software runs the finite element analysis together with adaptive meshing and error control using a variety of numerical solvers. The photoreactor modeling requires transportreaction coupling which exists in COMSOL Multiphysics Chemical Engineering Module and Reaction Engineering Lab. The COMSOL Reaction Engineering Lab® is a tool that uses reaction formulas (kinetic expressions) to create models involving momentum, mass, and energy in COMSOL Multiphysics. 


\section{Chapter 4}

\section{RESULTS AND DISCUSSION}

In this chapter, reaction mechanism, mechanistic kinetic model, experimental design, statistical analysis, optimization, photoreactor modeling, kinetic parameter estimation, sensitivity analysis, scaling-up methodology, and CFD modeling for different AOTs are presented.

In Section 4.1, the kinetic models for the photo-oxidative degradation of poly(ethylene glycol) (PEG) are developed for the process of ultraviolet radiation and hydrogen peroxide $\left(\mathrm{UV} / \mathrm{H}_{2} \mathrm{O}_{2}\right)$. The rate expressions of the photochemical degradation of soluble polymers are developed based on the mass balance of the main chemical species in water. Continuous-distribution kinetics is applied in kinetic modeling of the photo-oxidative degradation of polymers in aqueous solution based on the population balance equations (PBEs). The random chain scission is assumed to be the mechanism of the chain cleavage. The PBEs are solved by the moment operation which transforms the integro-differential equations into ordinary differential equations that could be readily solved to obtain the rate coefficients of the polymer photodegradation. The model predictions for the number average molecular weight and the number of chain scissions per molecule are presented and compared with the experimental data obtained from the open literature for the photodegradation of PEG by $\mathrm{UV} / \mathrm{H}_{2} \mathrm{O}_{2}$ in aqueous solution. Sequential quadratic programming (SQP) is used as an optimization technique to find the kinetic parameters that can be used for scaling-up purposes.

In Section 4.2, advanced oxidation of aqueous poly(ethylene oxide) (PEO) was studied using the $\mathrm{UV} / \mathrm{H}_{2} \mathrm{O}_{2}$ process in a batch recirculation photoreactor. The response surface methodology 
(RSM) combined with quadratic programming was used for the experimental design, statistical analysis, and optimization of the process. In the second part, a detailed mathematical model is developed to predict the total organic carbon (TOC) removal as a function of time. Continuous distribution kinetics is applied to establish the kinetic model for the photodegradation of PEO. The model is checked for validation at different influential operating conditions using experimental data obtained by a recirculation batch photoreactor. Also, the intrinsic rate constants are estimated using an optimization algorithm.

In Section 4.3, the application of a Box-Behnken experimental design combined with RSM and quadratic programming was investigated for the photo-Fenton-like degradation of poly(acrylic acid) (PAA) in aqueous solution. Also, a mechanistic kinetic model for the photo-Fenton-like degradation of PAA is developed and experimentally validated. The developed mathematical model incorporates all photochemical reactions including $\mathrm{Fe}^{2+}$ and $\mathrm{Fe}^{3+}$ chlorocomplexes, inorganic anion radicals $\left(\mathrm{Cl}_{2}^{--}\right)$, and polymer degradation. Population balance equations are used for kinetic modeling of the polymer degradation based on the chain-end scission. The proposed model could accurately predict the TOC removal rate of six sets of experimental runs. Sensitivity analysis is also performed to evaluate the most significant kinetic parameters used in the mathematical model. Furthermore, an experimental run is conducted in continuous mode at optimum operating conditions to investigate the computational fluid dynamics (CFD) based on the transport-reaction coupling. The CFD simulation result for the TOC removal is compared with the experimental data. The low discrepancy between CFD simulation result and experimental data confirms the reliability of the CFD model.

In Section 4.4, the effectiveness of the photo-Fenton-like process to degrade aqueous PAA was investigated in a batch recirculation system using two photoreactors in series. The response 
surface methodology using Box-Behnken experimental design combined with quadratic programming was employed for experimental design, statistical analysis, and optimization. The effects of the initial concentration of PAA, the initial concentration of $\mathrm{H}_{2} \mathrm{O}_{2}: \mathrm{Fe}^{3+}$, $\mathrm{pH}$, and the recirculation rate on the percent total organic carbon (TOC) removal and the "pseudo"-second order rate constant were studied. The statistical analysis of results indicated satisfactory prediction of the system behavior by the derived quadratic models. Optimum operating conditions to maximize TOC removal were also determined.

In Section 4.5, a scaling-up methodology is developed in order to scale up the photodegradation process of poly(vinyl alcohol) (PVA) from the laboratory-scale photoreactor to a pilot-scale one with different geometry and configuration avoiding intermediate steps. In this procedure, the intrinsic kinetic parameters are determined using a laboratory-scale photoreactor. The obtained kinetic parameters are used to predict the performance of the pilot-scale continuous flow through the photoreactor without any adjustable parameter. In both cases, mass and radiation balances are needed. However, for the pilot-scale photoreactor, the three-dimensional radiation balance and differential mass balance have to be developed. The model predictions using CFD model development is validated in a pilot-scale photoreactor at different operating conditions. 


\subsection{KINETIC STUDY OF PHOTODEGRADATION OF WATER- SOLUBLE POLYMERS}

\subsubsection{Introduction}

In this section, the mechanisms and kinetic modeling of the photo-oxidative degradation of water-soluble polymers in water by the $\mathrm{UV} / \mathrm{H}_{2} \mathrm{O}_{2}$ process are investigated. There is little information available in the open literature regarding the detailed kinetic modeling of such soluble polymers by AOTs considering the radical and chemical species present in water. PEG was chosen as the model water-soluble polymer. A detailed mathematical model constituted by the mass balance of the main species present in water is developed. Radical depolymerization for the photo-oxidative degradation of synthetic water-soluble polymers in solution is mathematically modeled using population balance equations. The moment method is applied to solve the population balance equations. The model predictions are validated using the experimental data from the open literature (Santos et al., 2009). Finally, a nonlinear least-square objective function is used to find the model parameters. This can be considered as the starting point for designing commercial-scale photoreactors.

\subsubsection{Reaction Mechanisms}

As mentioned earlier, the reaction mechanisms often used to describe the degradation of macromolecules comprise three reversible elementary steps including initiation-termination of radicals, depropagation (scission, decay), and hydrogen abstraction. According to the long chain approximation (LCA), initiation-termination steps are neglected because of the insignificant effect on the overall rate of reactions. In this study, random chain scission is considered as the 
mechanism of the degradation. Therefore, the oxidizing radicals, generated by UV irradiation, initiate the reaction by hydrogen abstraction from a polymer molecule to generate random and specific end polymer radicals. In this study, the UV photolysis at $254 \mathrm{~nm}$ results in $\mathrm{H}_{2} \mathrm{O}_{2}$ decomposition into free radicals as shown in Table 4.1 (Ghafoori et al., 2012a).

Table 4.1. Reaction mechanisms of the photodegradation in $\mathrm{UV} / \mathrm{H}_{2} \mathrm{O}_{2}$ process.

\begin{tabular}{|c|c|c|c|}
\hline No. & Reaction & Rate Constant & References \\
\hline R1 & $\mathrm{H}_{2} \mathrm{O}_{2} \stackrel{\varphi_{\mathrm{H}_{2} \mathrm{O}_{2}}, h v}{\longrightarrow} 2 \cdot \mathrm{OH}$ & $0.5\left(\right.$ mol Einstein $\left.^{-1}\right)$ & Crittenden et al., 1999 \\
\hline $\mathrm{R} 2$ & $\cdot \mathrm{OH}+\mathrm{H}_{2} \mathrm{O}_{2} \stackrel{k_{2}}{\rightarrow} \mathrm{HO}_{2}+\mathrm{H}_{2} \mathrm{O}$ & $2.7 \times 10^{7} \quad\left(\mathrm{M}^{-1} \mathrm{~s}^{-1}\right)$ & Buxton et al., 1988 \\
\hline R3 & $\cdot \mathrm{OH}+\mathrm{HO}_{2}^{-} \stackrel{k_{3}}{\rightarrow} \mathrm{HO}_{2}^{\cdot}+\mathrm{OH}^{-}$ & $7.5 \times 10^{9} \quad\left(\mathrm{M}^{-1} \mathrm{~s}^{-1}\right)$ & Christensen et al., 1982 \\
\hline $\mathrm{R} 4$ & $\mathrm{HO}_{2}+\mathrm{H}_{2} \mathrm{O}_{2} \stackrel{k_{4}}{\rightarrow} \cdot \mathrm{OH}+\mathrm{H}_{2} \mathrm{O}+\mathrm{O}_{2}$ & $\left(\mathrm{M}^{-1} \mathrm{~s}^{-1}\right)$ & Koppenol et al., 1978 \\
\hline R5 & $\mathrm{O}_{2}^{--}+\mathrm{H}_{2} \mathrm{O}_{2} \stackrel{k_{5}}{\rightarrow} \cdot \mathrm{OH}+\mathrm{O}_{2}+\mathrm{OH}^{-}$ & $\left(\mathrm{M}^{-1} \mathrm{~s}^{-1}\right)$ & Weinstein and Bielski, 1979 \\
\hline R6 & $\mathrm{O}_{2}^{\cdot-}+\mathrm{H}^{+} \stackrel{k_{6}}{\rightarrow} \mathrm{HO}_{2}^{\cdot}$ & $\left(\mathrm{M}^{-1} \mathrm{~s}^{-1}\right)$ & Bielski et al., 1985 \\
\hline R7 & $\mathrm{HO}_{2}^{\cdot} \stackrel{k_{7}}{\rightarrow} \mathrm{O}_{2}^{--}+H^{+}$ & $1.58 \times 10^{5}\left(\mathrm{~s}^{-1}\right)$ & Bielski et al., 1985 \\
\hline $\mathrm{R} 8$ & 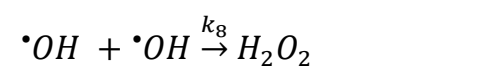 & $5.5 \times 10^{9} \quad\left(\mathrm{M}^{-1} \mathrm{~s}^{-1}\right)$ & Buxton et al., 1988 \\
\hline R9 & $\mathrm{HO}_{2}^{\cdot}+\mathrm{HO}_{2}^{\cdot} \stackrel{k_{9}}{\rightarrow} \mathrm{H}_{2} \mathrm{O}_{2}+\mathrm{O}_{2}$ & $8.3 \times 10^{5} \quad\left(\mathrm{M}^{-1} \mathrm{~s}^{-1}\right)$ & Bielski et al., 1985 \\
\hline R10 & $\cdot \mathrm{OH}+\mathrm{HO}_{2}^{\cdot} \stackrel{k_{10}}{\longrightarrow} \mathrm{O}_{2}+\mathrm{H}_{2} \mathrm{O}$ & $6.6 \times 10^{9} \quad\left(\mathrm{M}^{-1} \mathrm{~s}^{-1}\right)$ & Elliot and Buxton, 1992 \\
\hline R11 & $\mathrm{HO}_{2}^{\cdot}+\mathrm{O}_{2}^{--} \stackrel{k_{11}}{\rightarrow} \mathrm{HO}_{2}^{-}+\mathrm{O}_{2}$ & $9.7 \times 10^{7} \quad\left(\mathrm{M}^{-1} \mathrm{~s}^{-1}\right)$ & Bielski et al., 1985 \\
\hline R12 & $\cdot \mathrm{OH}+\mathrm{O}_{2}^{--} \stackrel{k_{12}}{\longrightarrow} \mathrm{O}_{2}+\mathrm{OH}^{-}$ & $8 \times 10^{9} \quad\left(\mathrm{M}^{-1} \mathrm{~s}^{-1}\right)$ & Linden et al., 2005 \\
\hline R13 & $\mathrm{H}_{2} \mathrm{O}_{2} \rightleftharpoons \mathrm{H}^{+}+\mathrm{HO}_{2}^{-}$ & $\mathrm{p} K_{a}=11.6$ & Perry et al., 1981 \\
\hline
\end{tabular}


The hydrogen abstraction of polymer chain $P_{n}$ to generate random radicals by ${ }^{\bullet} \mathrm{OH}$ and $\mathrm{HO}_{2}^{\bullet}$ can be described by Reactions (4.1) and (4.2) (McCoy and Madras, 1997):

$P_{n}+\cdot O H \stackrel{k_{h a O}}{\longrightarrow} R_{n}^{\bullet}+H_{2} \mathrm{O}$

$P_{n}+\mathrm{HO}_{2}^{\cdot} \stackrel{k_{\text {hao }}^{\prime}}{\longrightarrow} R_{n}^{\cdot}+\mathrm{H}_{2} \mathrm{O}$

where $k_{\text {hao }}$ and $k_{\text {hao }}^{\prime}$ represent rate constants for the hydrogen abstraction to generate random radicals by oxidizing ${ }^{\circ} \mathrm{OH}$ and $\mathrm{HO}_{2}^{\bullet}$ radicals, respectively. The radicals become stabilized by abstracting a hydrogen atom from a stable position. The reversible intermolecular hydrogen abstraction of a polymer chain to give a random radical can be represented by Reaction (4.3):

$P_{n} \stackrel{k_{h a}}{\underset{k_{\mathrm{Ha}}}{\rightleftharpoons}} R_{n}^{\cdot}$

where $k_{h a}$ and $k_{H a}$ represent the rate constants for the hydrogen abstraction from a polymer to give a random radical and reversible hydrogen abstraction from a random radical, respectively. The depropagation reaction of a random radical is given as follows (Smagala and McCoy, 2003).

$R_{n}^{\cdot} \stackrel{k_{d}}{\rightarrow} R^{\bullet}\left(n^{\prime}\right)+P\left(n-n^{\prime}\right)$

where $k_{d}$ represents the degradation coefficient for the depropagation of the random radical.

\subsubsection{Kinetic Model Development}

Chemical species of the reacting polymer and radicals of molecular $n$ are represented as $P_{n}, R_{n}^{\bullet}$, and their molecular weight distributions (MWDs) as $p(n, t)$ and $r(n, t)$, respectively. Since the polymer reactants and scission products cannot be distinguished in the continuous-distribution model, a single MWD, $p(n, t)$, represents the polymer mixture at any time. The time dependent 
MWD, denoted as $p(n, t)$, is defined so that $p(n, t) \mathrm{d} n$ is the molar concentration of polymer in the molecular weight range $(n, n+\mathrm{d} n)$. It is useful to distinguish between the reactant and product MWDs by writing separate governing differential equations for their behaviour (Konaganti and Madras, 2009). As mentioned in Section 2.2.3.1, for a binary fragmentation reaction, the rate of formation of the products of a binary scission is presented by Equation (4.5) in which the integral is used instead of the sum to make the calculations easier:

$R(n)=\int_{n}^{\infty} k\left(n^{\prime}\right) \Omega\left(n, n^{\prime}\right) r\left(n^{\prime}, t\right) \mathrm{d} n^{\prime}$

The stoichiometric coefficient $\Omega\left(n, n^{\prime}\right)$ represents the likelihood of obtaining a scission product of molecular weight $x$ from a larger molecule $n^{\prime}$. In other words, it indicates the reaction in which a molecule breaks into two product molecules of $n$ and $n^{\prime}-n$ sizes, where $n^{\prime} \geq n$ and the $k\left(n^{\prime}\right)$ is the rate coefficient. A general expression for the stoichiometric coefficient is given by equation (4.6):

$\Omega\left(n, n^{\prime}\right)=\frac{n^{m}\left(n^{\prime}-n\right)^{m} \Gamma(2 m+2)}{\Gamma(m+1)^{2}\left(n^{\prime}\right)^{2 m+1}}$

where $\Gamma$ is the gamma function and $m$ is a parameter indicating the shape of the scission fragment distribution, respectively. For the case of random scission, $m=0$ and $\Omega\left(n, n^{\prime}\right)=1 / n^{\prime}$. Therefore, the kinetic model for the main chemical species present in water can be developed. The rate of disappearance of the oxidizer by direct photolysis could be expressed by equation (4.7). The direct photolysis of the PEG is not considered as it is negligible compared to the direct photolysis of $\mathrm{H}_{2} \mathrm{O}_{2}$.

$\frac{d C_{U V, H_{2} O_{2}}}{d t}=-\varphi_{H_{2} O_{2}} e_{\lambda}^{a}$ 
where $\varphi_{\mathrm{H}_{2} \mathrm{O}_{2}}$ is the quantum yield of acid-base conjugates $\mathrm{H}_{2} \mathrm{O}_{2}$ and $\mathrm{HO}_{2}^{-}\left(\right.$mol Einstein $\left.{ }^{-1}\right)$ and $e_{\lambda}^{a}$ is the local volumetric rate of energy absorption (LVREA) (Einstein $\mathrm{L}^{-1} \mathrm{~s}^{-1}$ ) that is defined according to the Beer-Lambert law that is based on the radiation balance as defined below:

$$
\begin{aligned}
& e_{\lambda}^{a}= \\
& I_{O}\left(\frac{\left.\varepsilon_{\mathrm{H}_{2} \mathrm{O}_{2}\left[\mathrm{H}_{2} \mathrm{O}_{2}\right]+\varepsilon_{\mathrm{HO}_{2}^{-}}^{-}\left[\mathrm{HO}_{2}^{-}\right]}^{\varepsilon_{\mathrm{H}_{2} \mathrm{O}_{2}}\left[\mathrm{H}_{2} \mathrm{O}_{2}\right]+\varepsilon_{\mathrm{HO}_{2}^{-}}\left[\mathrm{HO}_{2}^{-}\right]+\varepsilon_{T O C_{1}}\left[\mathrm{TOC}_{1}\right]}\right)}{\left(1-e^{\left(-2.303 b\left(\varepsilon_{\mathrm{H}_{2} \mathrm{O}_{2}}\left[\mathrm{H}_{2} \mathrm{O}_{2}\right]+\varepsilon_{\mathrm{HO}_{2}^{-}}\left[\mathrm{HO}_{2}^{-}\right]+\varepsilon_{T O C_{1}}\left[\mathrm{TOC}_{1}\right]\right)\right)}\right)}\right.
\end{aligned}
$$

where $I_{o}$, the UV light intensity absorbed by the solution, was calculated to be $1.63 \times 10^{-5}$ Einstein $\mathrm{L}^{-1} \mathrm{~s}^{-1}$, which is the incident $254 \mathrm{~nm}$ photon irradiance estimated from $96 \mathrm{~W}$ LP lamp over the photoreactor. The fraction shows the portion of the UV radiation absorption by hydrogen peroxide. Parameters $\varepsilon_{\mathrm{H}_{2} \mathrm{O}_{2}}, \varepsilon_{\mathrm{HO}_{2}^{-}}$, and $\varepsilon_{P E G}$ are the molar extinction coefficients of $\mathrm{H}_{2} \mathrm{O}_{2}(18.7$ $\left.\mathrm{M}^{-1} \mathrm{~cm}^{-1}\right), \quad \mathrm{HO}_{2}^{-}\left(210 \mathrm{M}^{-1} \mathrm{~cm}^{-1}\right)$, and PEG $\left(118.8 \mathrm{M}^{-1} \mathrm{~cm}^{-1}\right)$ at $254 \mathrm{~nm}$, respectively. The parameter $b$ is the effective path length of the photoreactor $(2 \mathrm{~cm})$.

$\mathrm{H}_{2} \mathrm{O}_{2}$ and $\mathrm{HO}_{2}^{-}$are interrelated through the equilibrium Reaction (R13) in Table 4.1. The total concentration of $\mathrm{H}_{2} \mathrm{O}_{2}$ is defined as follows:

$\left[\mathrm{H}_{2} \mathrm{O}_{2}\right]_{T}=\left[\mathrm{H}_{2} \mathrm{O}_{2}\right]+\left[\mathrm{HO}_{2}^{-}\right]$

Consequently, a new equilibrium concentration for the two individual species can be expressed by the following equations:

$\left[\mathrm{H}_{2} \mathrm{O}_{2}\right]=\frac{\left[\mathrm{H}_{2} \mathrm{O}_{2}\right]_{T}}{1+K_{a}\left[\mathrm{H}^{+}\right]^{-1}}$ 
$\left[\mathrm{HO}_{2}^{-}\right]=\frac{\left[\mathrm{H}_{2} \mathrm{O}_{2}\right]_{T}}{1+K_{a}^{-1}\left[H^{+}\right]}$

where $K_{a}$ is the equilibrium rate constant. Therefore, these concentrations are used later in developing the kinetic model. All other kinetic expressions are obtained by incorporating the decomposition and the production of the species through free-radical reactions as shown below:

$$
\begin{aligned}
& R_{\mathrm{H}_{2} \mathrm{O}_{2}}= \\
& \left(-R_{U V, H_{2} \mathrm{O}_{2}}-k_{2}\left[^{\bullet} \mathrm{OH}\right]\left[\mathrm{H}_{2} \mathrm{O}_{2}\right]-k_{3}\left[^{\bullet} \mathrm{OH}\right]\left[\mathrm{HO}_{2}^{-}\right]-k_{4}\left[\mathrm{HO}_{2}^{\bullet}\right]\left[\mathrm{H}_{2} \mathrm{O}_{2}\right]-k_{5}\left[\mathrm{O}_{2}^{\bullet-}\right]\left[\mathrm{H}_{2} \mathrm{O}_{2}\right]+\right. \\
& \left.k_{8}\left[{ }^{\bullet} \mathrm{OH}\right]^{2}+k_{9}\left[H O_{2}^{\bullet}\right]^{2}+k_{11}\left[H O_{2}^{\bullet}\right]\left[\mathrm{O}_{2}^{\bullet-}\right]\right) \times\left(\frac{1}{1+K_{a}\left[H^{+}\right]^{-1}}\right) \\
& R_{\mathrm{HO}_{2}^{-}}= \\
& \left(-R_{U V, \mathrm{H}_{2} \mathrm{O}_{2}}-k_{2}\left[^{\bullet} \mathrm{OH}\right]\left[\mathrm{H}_{2} \mathrm{O}_{2}\right]-k_{3}\left[^{\bullet} \mathrm{OH}\right]\left[\mathrm{HO}_{2}^{-}\right]-k_{4}\left[\mathrm{HO}_{2}^{\bullet}\right]\left[\mathrm{H}_{2} \mathrm{O}_{2}\right]-k_{5}\left[\mathrm{O}_{2}^{\bullet-}\right]\left[\mathrm{H}_{2} \mathrm{O}_{2}\right]+\right. \\
& \left.k_{8}\left[{ }^{\bullet} \mathrm{OH}\right]^{2}+k_{9}\left[\mathrm{HO}_{2}^{\bullet}\right]^{2}+k_{11}\left[\mathrm{HO}_{2}^{\bullet}\right]\left[\mathrm{O}_{2}^{\bullet-}\right]\right) \times\left(\frac{1}{1+K_{a}^{-1}\left[H^{+}\right]}\right) \\
& R \cdot \bullet_{\mathrm{OH}}=2 R_{U V, \mathrm{H}_{2} \mathrm{O}_{2}}-k_{2}\left[^{\cdot} \mathrm{OH}\right]\left[\mathrm{H}_{2} \mathrm{O}_{2}\right]-k_{3}\left[{ }^{\bullet} \mathrm{OH}\right]\left[\mathrm{HO}_{2}^{-}\right]+k_{4}\left[\mathrm{HO}_{2}^{\bullet}\right]\left[\mathrm{H}_{2} \mathrm{O}_{2}\right]+ \\
& k_{5}\left[\mathrm{O}_{2}^{\bullet-}\right]\left[\mathrm{H}_{2} \mathrm{O}_{2}\right]-k_{8}\left[^{\bullet} \mathrm{OH}\right]^{2}-k_{10}\left[^{\circ} \mathrm{OH}\right]\left[\mathrm{HO}_{2}^{\bullet}\right]-k_{12}\left[^{\bullet} \mathrm{OH}\right]\left[\mathrm{O}_{2}^{--}\right]- \\
& {\left[{ }^{\bullet} \mathrm{OH}\right]\left(\int_{0}^{\infty} k_{\text {hao }}\left(n^{\prime}\right) p\left(n^{\prime}, t\right) \mathrm{d} n^{\prime}\right)} \\
& R_{\mathrm{HO}_{2}^{\circ}}=k_{2}\left[{ }^{\bullet} \mathrm{OH}\right]\left[\mathrm{H}_{2} \mathrm{O}_{2}\right]+k_{3}\left[{ }^{\bullet} \mathrm{OH}\right]\left[\mathrm{HO}_{2}^{-}\right]-k_{4}\left[\mathrm{HO}_{2}^{\bullet}\right]\left[\mathrm{H}_{2} \mathrm{O}_{2}\right]+k_{6}\left[\mathrm{O}_{2}^{\bullet-}\right]\left[\mathrm{H}^{+}\right]-k_{7}\left[\mathrm{HO}_{2}^{\bullet}\right]- \\
& k_{9}\left[\mathrm{HO}_{2}^{*}\right]^{2}-k_{10}\left[{ }^{\bullet} \mathrm{OH}\right]\left[\mathrm{HO}_{2}^{*}\right]-k_{11}\left[\mathrm{HO}_{2}^{*}\right]\left[\mathrm{O}_{2}^{\bullet-}\right]-\left[\mathrm{HO}_{2}^{\bullet}\right]\left(\int_{0}^{\infty} k_{\text {hao }}^{\prime}\left(n^{\prime}\right) p\left(n^{\prime}, t\right) \mathrm{d} n^{\prime}\right) \\
& R_{\mathrm{O}_{2}^{--}}=-k_{5}\left[\mathrm{O}_{2}^{\bullet-}\right]\left[\mathrm{H}_{2} \mathrm{O}_{2}\right]-k_{6}\left[\mathrm{O}_{2}^{\bullet-}\right]\left[\mathrm{H}^{+}\right]+k_{7}\left[\mathrm{HO}_{2}^{\bullet}\right]-k_{11}\left[\mathrm{HO}_{2}^{\bullet}\right]\left[\mathrm{O}_{2}^{\bullet-}\right]-k_{12}\left[{ }^{\bullet} \mathrm{OH}\right]\left[\mathrm{O}_{2}^{\bullet-}\right]
\end{aligned}
$$


The integral parts represent the hydrogen abstraction reaction between ${ }^{\circ} \mathrm{OH}$ and $\mathrm{HO}_{2}^{\bullet}$ radicals and the polymer of any molecular weight. In the developed model, $\mathrm{pH}$ is considered to be constant as it is proven that $\mathrm{pH}$ changes during the process do not have significant effect on the model predictions (Crittenden et al., 1999). The population balance equations for polymer and polymer radicals can be written as follows:

$$
\begin{aligned}
& R_{p}(n, t)=-k_{h a o}(n)\left[{ }^{\bullet} \mathrm{OH}\right] p(n, t)-k_{\text {hao }}^{\prime}(n)\left[H O_{2}^{*}\right] p(n, t)-k_{h a}(n) p(n, t)+k_{H A}(n) r(n, t)+ \\
& \int_{x}^{\infty} k_{d}\left(n^{\prime}\right) r\left(n^{\prime}, t\right) \Omega\left(n, n^{\prime}\right) \mathrm{d} n^{\prime} \\
& R_{r}(n, t)=k_{h a o}(n)\left[{ }^{\bullet} O H\right] p(n, t)+k_{h a o}^{\prime}(n)\left[H O_{2}^{*}\right] p(n, t)+k_{h a}(n) p(n, t)-k_{H A}(n) r(n, t)+ \\
& \int_{x}^{\infty} k_{d}\left(n^{\prime}\right) r\left(n^{\prime}, t\right) \Omega\left(n, n^{\prime}\right) \mathrm{d} n^{\prime}
\end{aligned}
$$

At small reaction times, all rate coefficients are usually assumed independent of molecular weight and they could be considered constant (Madras et al., 1997).

\subsubsection{Solution Method}

The moment operation is applied to transform the integro-differential equations to ordinary differential equations. The moments of the MWDs are defined as integrals over the molecular weight, $x$ :

$$
r^{(p)}(t)=\int_{0}^{\infty} r(n, t) x^{p} \mathrm{~d} n
$$

The zero moment $(p=0)$ is the time-dependent total molar concentration of the polymer and the first moment $(p=1)$ is the mass concentration of the polymer. By applying moment operations, 
the integro-differential Equations (4.17) and (4.18) result in the following ordinary differential equations:

$$
\begin{aligned}
& \frac{\mathrm{d} p^{(p)}}{\mathrm{d} t}=-k_{\text {hao }}[\bullet O H] p^{(p)}-k_{h a o}^{\prime}\left[H O_{2}^{\bullet}\right] p^{(p)}-k_{h a} p^{(p)}+k_{H A} r^{(p)}-\frac{1}{n+1} k_{d} r^{(p)} \\
& \frac{\mathrm{d} r^{(p)}}{\mathrm{d} t}=-k_{\text {hao }}[\cdot O H] p^{(p)}+k_{h a o}^{\prime}\left[H O_{2}^{\bullet}\right] p^{(p)}+k_{h a} p^{(p)}-k_{H A} r^{(p)}-\frac{n}{n+1} k_{d} r^{(p)}
\end{aligned}
$$

By applying the quasi-steady state approximation to random radicals (Equation (4.21)) for zeroth and first moments, the following equations are obtained:

$$
\begin{aligned}
\frac{\mathrm{d} p^{(0)}}{\mathrm{d} t} & =\left(k_{A}\left[{ }^{\bullet} \mathrm{OH}\right]+k_{B}\left[H O_{2}^{\bullet}\right]+k_{C}\right) p^{(0)} \\
\frac{\mathrm{d} p^{(1)}}{\mathrm{d} t} & =0
\end{aligned}
$$

where: $\quad k_{A}=\frac{k_{d} k_{\text {hao }}}{k_{H A}}, \quad k_{B}=\frac{k_{d} k_{\text {hao }}^{\prime}}{k_{H A}}, \quad$ and $\quad k_{C}=\frac{k_{h a} k_{d}}{k_{H A}}$

The rate of change of the first moment is zero $\left(\frac{d p^{(1)}}{d t}=0\right)$, which confirms the mass conservation.

The rate of change of oxidizing radicals can be shown by rearranging Equations (4.14) and (4.15) as follows:

$$
\begin{aligned}
& R_{\bullet} \mathrm{OH} \\
& =2 R_{U V, \mathrm{H}_{2} \mathrm{O}_{2}}-k_{2}\left[^{\bullet} \mathrm{OH}\right]\left[\mathrm{H}_{2} \mathrm{O}_{2}\right]-k_{3}\left[\bullet^{\bullet} \mathrm{OH}\right]\left[\mathrm{HO}_{2}^{-}\right]+k_{4}\left[\mathrm{HO}_{2}^{\bullet}\right]\left[\mathrm{H}_{2} \mathrm{O}_{2}\right]+ \\
& k_{5}\left[\mathrm{O}_{2}^{\bullet-}\right]\left[\mathrm{H}_{2} \mathrm{O}_{2}\right]-k_{8}\left[{ }^{\bullet} \mathrm{OH}\right]^{2}-k_{10}\left[{ }^{\bullet} \mathrm{OH}\right]\left[\mathrm{HO}_{2}^{\bullet}\right]-k_{12}\left[{ }^{\bullet} \mathrm{OH}\right]\left[\mathrm{O}_{2}^{\bullet-}\right]-\left[{ }^{\bullet} \mathrm{OH}\right] k_{\text {hao }} p^{(0)} \\
& R_{\mathrm{HO}_{2}^{\bullet}}=k_{2}\left[{ }^{\bullet} \mathrm{OH}\right]\left[\mathrm{H}_{2} \mathrm{O}_{2}\right]+k_{3}\left[^{\bullet} \mathrm{OH}\right]\left[\mathrm{HO}_{2}^{-}\right]-k_{4}\left[\mathrm{HO}_{2}^{\bullet}\right]\left[\mathrm{H}_{2} \mathrm{O}_{2}\right]+k_{6}\left[\mathrm{O}_{2}^{\bullet-}\right]\left[\mathrm{H}^{+}\right]-k_{7}\left[\mathrm{HO}_{2}^{\bullet}\right]- \\
& k_{9}\left[\mathrm{HO}_{2}^{\bullet}\right]^{2}-k_{10}\left[^{\bullet} \mathrm{OH}\right]\left[\mathrm{HO}_{2}^{\bullet}\right]-k_{11}\left[\mathrm{HO}_{2}^{\bullet}\right]\left[\mathrm{O}_{2}^{\bullet-}\right]-\left[\mathrm{HO}_{2}^{\bullet}\right] k_{\text {hao }}^{\prime} p^{(0)}
\end{aligned}
$$


The profile of the number average molecular weight $\left(M_{n}\right)$, defined as $M_{n}=p^{(1)} / p^{(0)}$ could be obtained by numerically solving Equations (4.12), (4.13), (4.16), and (4.22) to (4.25) using "ode15s" in MATLAB 7.10 (R2010) program. In "ode15s", both the $4^{\text {th }}$ order Runge-Kutta method and backward differentiation formulas (Gear's method) are used. The computer program is provided in Appendix B. In order to validate the model, the model predictions have to be compared to the experimental data as shown in the next section.

\subsubsection{Model Validation}

A detailed mathematical model describing different steps of the free-radical induced degradation of PEG was developed. The model predictions are validated using the experimental data for photodegradation of poly(ethylene glycol) by the $\mathrm{UV} / \mathrm{H}_{2} \mathrm{O}_{2}$ process obtained from the open literature (Santos et al., 2009). The model parameters were chosen arbitrarily within reasonable limits to verify the model numerically. The results are shown in Figure 4.1 with a reasonable agreement between the experimental data and the model predictions for the number average molecular weight decay during the irradiation period. This figure also confirms the assumption of random chain scission mechanism, as in this type of chain scission, the molecular weight falls rapidly (Reich and Stivala, 1971). Also, this figure illustrates the predicted model for the number of chain scission per molecule $(S)$ that can be calculated from Equation (4.26) as follows:

$$
S=\frac{M_{n}(0)}{M_{n}(t)}-1
$$

$M_{n}(0)$ and $M_{n}(t)$ are the number average molecular weight of poly(ethylene glycol) before and after the time $(t)$ of the irradiation in minutes, respectively. As shown in Figure 4.1, the number 
of chain scission per molecule increases with the irradiation time which is in agreement with the exponential drop of the number average molecular weight, verifying a random chain scission mechanism. The goodness of fit between the model predictions and the actual values are presented in Figure 4.2 and Figure 4.3 for number average molecular weight and number of chain scissions per molecule, respectively. Figure 4.4 shows the simulation results for changes of hydrogen peroxide concentration and the total molar concentration of the polymer. As illustrated in this figure, during the course of the reactions, the concentration of hydrogen peroxide decreases as it is decomposed to hydroxyl radicals and hydroperoxyl radicals. These free radicals, as oxidizing agents, are mainly responsible to attack polymer molecules. Also, Figure 4.4 shows the exponential time behavior of the MWD moment (zero moment), which is the evidence for random chain scission, in a good agreement with the previous findings (Sterling and McCoy, 2001). On the other hand, by applying the quasi-steady state approximation, the effect of hydrogen abstraction in Equation (4.3) is de-emphasized and the degradation rate is controlled by Equations (4.1), (4.2) and (4.4). Therefore, full complement of the reactions and applying quasisteady state approximation result in increasing polymer molar concentration with time. Also, it is expected that by reducing the number average molecular weight and considering mass conservation law as shown in Equation (4.23), the total molar concentration of polymer increases with time that is confirmed in Figure 4.4. 


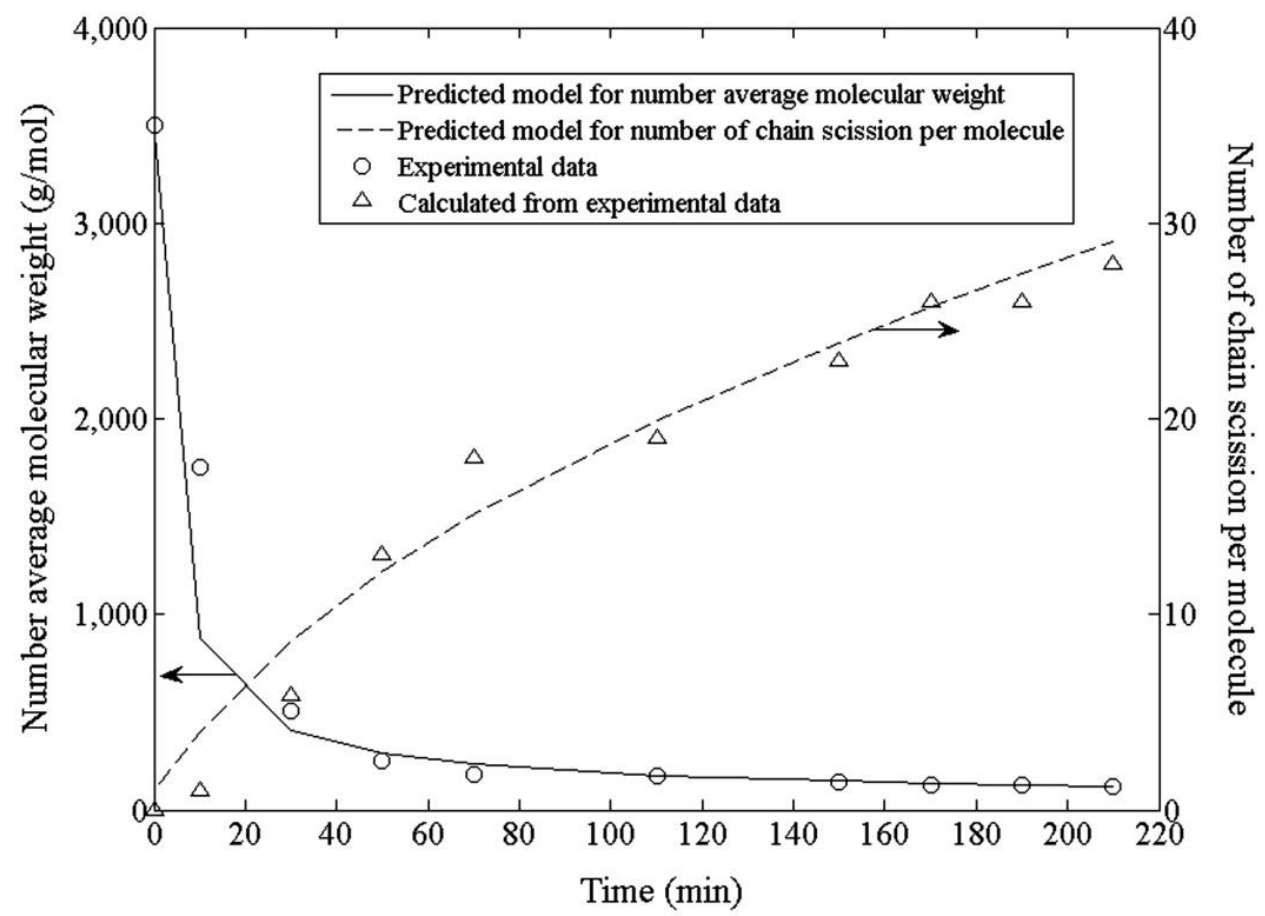

Figure 4.1. Comparison of the kinetic model predictions with the experimental data for number average molecular weight $\left(M_{n}\right)$ and number of chain scission per molecule $(S)$. 


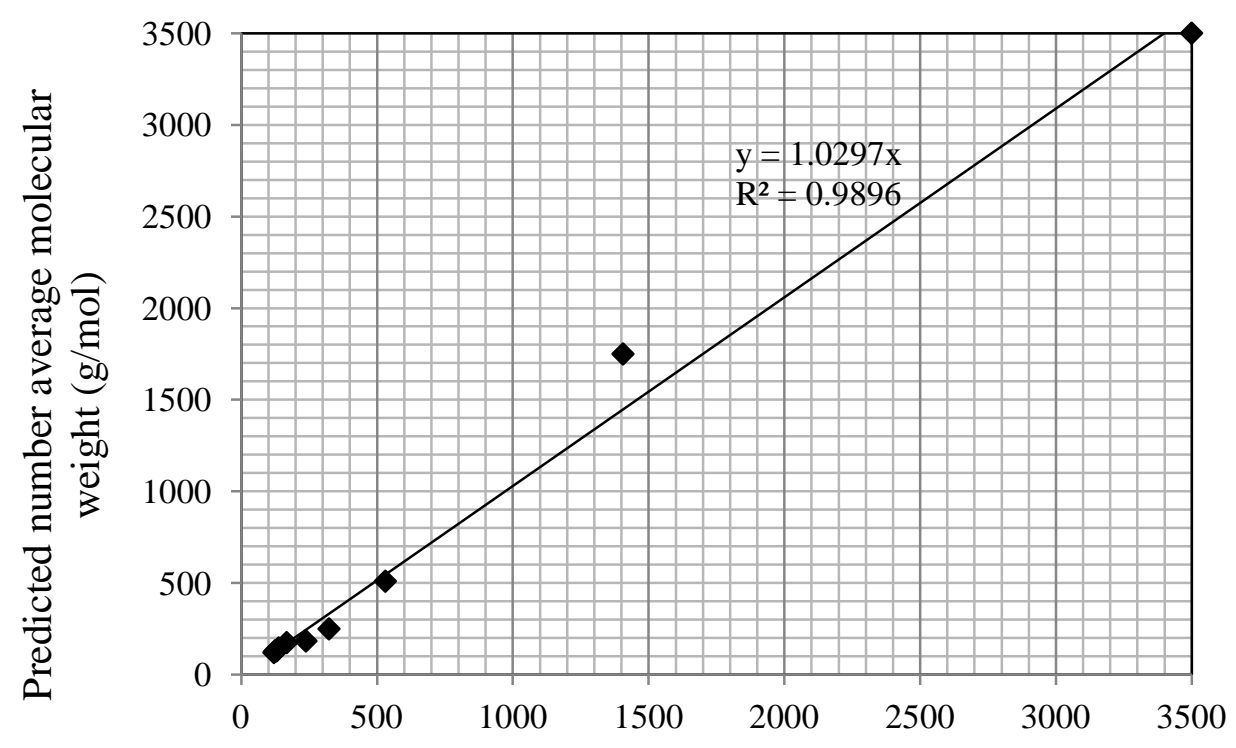

Actual number average molecular weight $(\mathrm{g} / \mathrm{mol})$

Figure 4.2. Comparison of predicted number average molecular weight versus actual values.

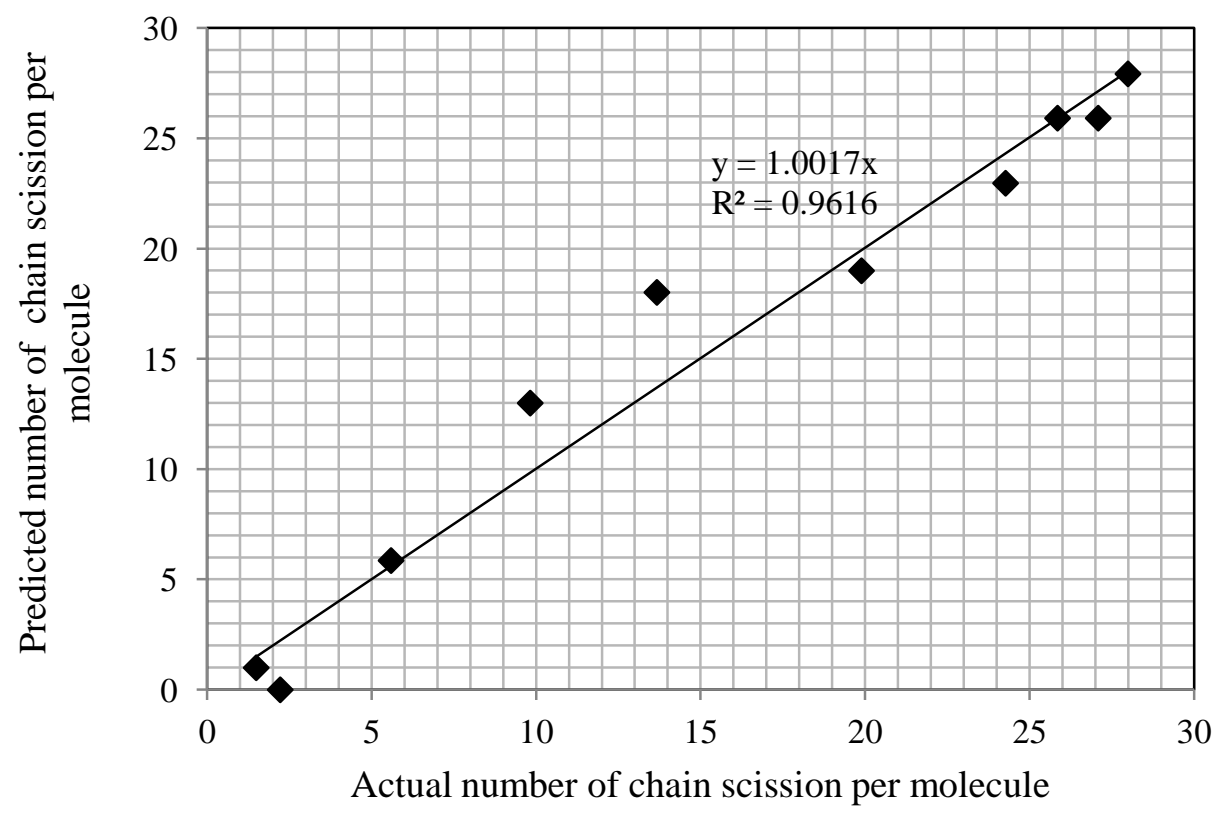

Figure 4.3. Comparison of predicted number average molecular weight versus actual values. 


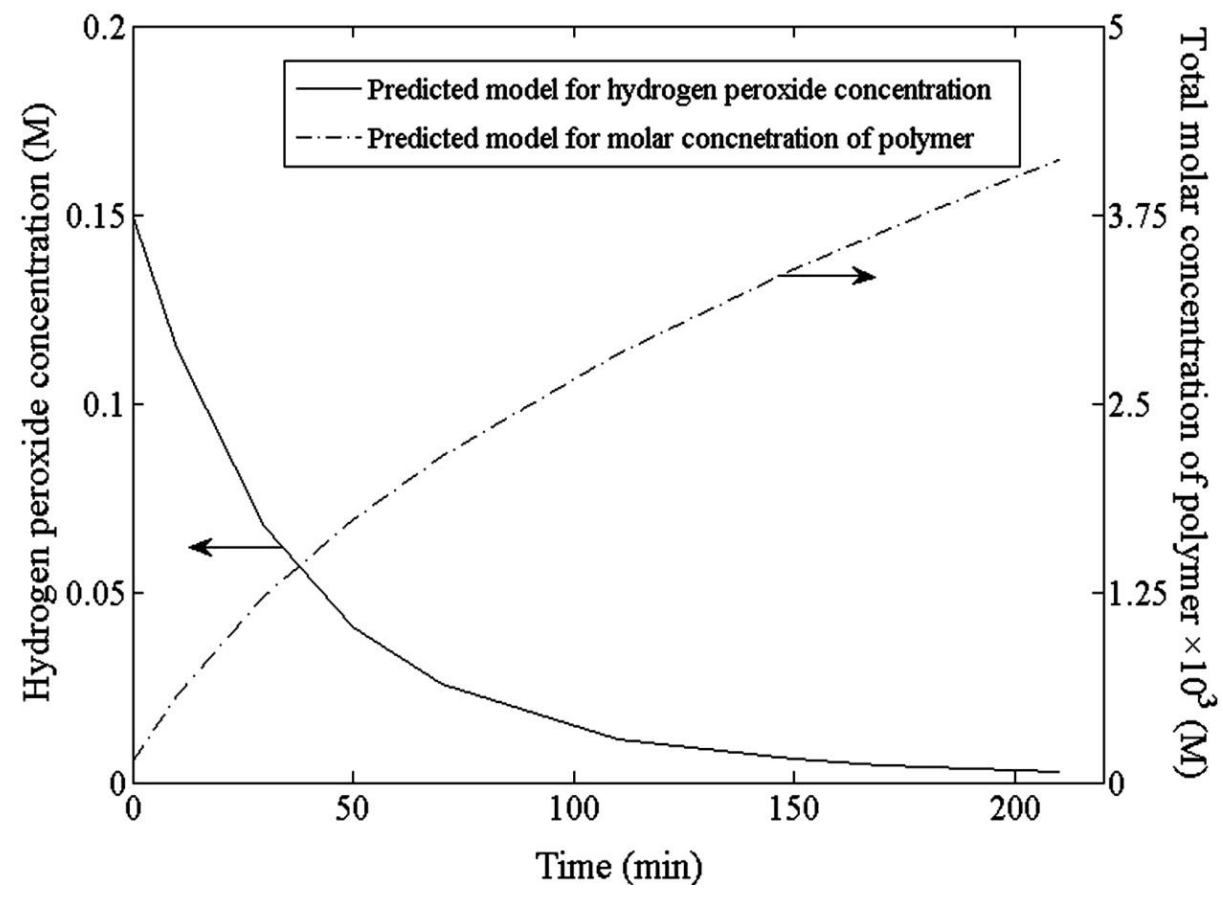

Figure 4.4. Simulation results for the concentration of hydrogen peroxide and total molar concentration of the polymer (zero moment). 


\subsubsection{Kinetic Parameter Estimation}

In the absence of underlying molecular weight results, approximations for the kinetic parameters could be estimated using an optimization algorithm to minimize the sum of squared errors as follows:

Minimize : $F(k)=\sum_{\mathrm{i}}\left(y_{i, m}-y_{i}(k)\right)^{2}$

where $y_{i, m}$ is the experimental data point from photodegradation experiments for $i$ number of data points and $y_{i}(k)$ is the model prediction with the vector of kinetic parameter $k$. Therefore, the goal is to find the values of $k$ that minimize $F(k)$ which must be subjected to kinetic model equations. The optimization was performed by MATLAB 7.10 (R2010) using sequential quadratic programming (SQP). Solving this problem results in obtaining optimal values of the

kinetic parameters. The optimal values of kinetic parameters $k_{A}, k_{B}, k_{C}, k_{h a o}$, and $k_{\text {hao }}^{\prime}$ were found to be $3.47 \times 10^{8} \mathrm{M}^{-1} \mathrm{~s}^{-1}, 14.23 \mathrm{M}^{-1} \mathrm{~s}^{-1}, 8.4 \times 10^{-5} \mathrm{~s}^{-1}, 4 \times 10^{9} \mathrm{M}^{-1} \mathrm{~s}^{-1}$, and $822.5 \mathrm{M}^{-1} \mathrm{~s}^{-1}$, respectively. However, in the gradient-based algorithm such as SQP, there is no test for determination of the global minimum. There might be many solutions to the optimization depending to the different starting points.

\subsubsection{Conclusions}

An accurate mathematical model for the photodegradation of PEG by the process of ultraviolet radiation and hydrogen peroxide was developed based on the mass balance of the main species present in water. The reactions were incorporated in a mathematical model for the polymer degradation. The kinetic modeling of polymer degradation was based on the population 
dynamics of the polymer molecule undergoing random chain scission. Moment operations were applied to make the models computationally inexpensive. The resulting models were a system of ordinary differential equations. The model was validated using the experimental data from the open literature. There was a good agreement between experimental data and the model prediction for both number average molecular weight and the number of chains scission per molecule. Increasing the number of chain scission per molecule and also exponential behavior of the zero moment with irradiation time confirmed the random chain scission assumption. The kinetic rate constants were determined by nonlinear parameter estimation using the SQP optimization algorithm. The optimal values of the kinetic parameters $k_{A}, k_{B}, k_{C}, k_{h a o}$, and $k_{\text {hao }}^{\prime}$ were found to be $3.47 \times 10^{8} \mathrm{M}^{-1} \mathrm{~s}^{-1}, 14.23 \mathrm{M}^{-1} \mathrm{~s}^{-1}, 8.4 \times 10^{-5} \mathrm{~s}^{-1}, 4 \times 10^{9} \mathrm{M}^{-1} \mathrm{~s}^{-1}$, and $822.5 \mathrm{M}^{-1} \mathrm{~s}^{-1}$, respectively. Obtaining the optimal values of the kinetic parameters that are independent of the reactor size and configuration can be considered as the starting point for designing commercial scale photoreactors. 


\subsection{FREE RADICAL-INDUCED DEGRADATION OF AQUEOUS \\ POLYETHYLENE OXIDE BY UV/H ${ }_{2} \mathrm{O}_{2}$ : EXPERIMENTAL DESIGN, REACTION MECHANISM, AND KINETIC MODELING}

\subsubsection{Introduction}

In the first part of this section, an experimental design is used to investigate the advanced oxidation of aqueous PEO as a synthetic water-soluble polymer, not amenable to biodegradation. Also, the effects of the initial PEO concentration, the initial $\mathrm{H}_{2} \mathrm{O}_{2}$ dosage, $\mathrm{pH}$, and the recirculation rate on the photodegradation of aqueous PEO by $\mathrm{UV} / \mathrm{H}_{2} \mathrm{O}_{2}$ process are investigated. The optimal values of parameters are determined by means of four-factor five-level central composite design (CCD) combined with response surface methodology (RSM) and quadratic programming. In the second part of this study, a mathematical model is developed to predict the percent TOC removal as the output variable with time in the $\mathrm{UV} / \mathrm{H}_{2} \mathrm{O}_{2}$ process. Continuous distribution kinetics that describes the dynamics of polymer reactions is applied to establish the TOC based kinetic modeling of the process. The developed model relies on the experimental data achieved in this work as well as the published data on the rate constants of the reaction between intermediates and hydroxyl radicals. Ultimately, the model is verified by another set of experimental data carried out at different operating conditions based on the results of the experimental design. Also, the intrinsic rate constants are estimated using an optimization algorithm. 


\subsubsection{Experimental Procedure}

The description and operating conditions of the laboratory-scale photoreactor (Figure 3.1) are given in Table 3.1. The $\mathrm{pH}$ was adjusted at the beginning of each experiment by adding few

drops of $1 \mathrm{~N} \mathrm{NaOH}$ or $\mathrm{H}_{2} \mathrm{SO}_{4}$ as needed and it was measured by a portable $\mathrm{pH}$ meter (230A plus, Thermo Orion). No further $\mathrm{pH}$ adjustment was performed during the experiment.

The following protocol was pursued in conducting each experiment: A PEO solution was prepared by gradually dissolving PEO in a $300-\mathrm{mL}$ beaker containing distilled water with a magnetic stirring bar at $50^{\circ} \mathrm{C}$ in order to avoid any foam formation. The solution was then diluted to achieve the desired PEO concentration in a 5-L solution and desired amount of $\mathrm{H}_{2} \mathrm{O}_{2}$ was added to the solution at the beginning of each experiment. The UV lamp was then turned on for 30 min before the beginning of each experiment to stabilize the light intensity and to degrade any possible organic background or micro-contaminant such as natural organic matter. The solution was fed to the system and the temperature was kept constant at $22^{\circ} \mathrm{C}$ during each experiment by means of a heat exchanger. The samples were taken from the collection tank at time 0 and at time interval of $30 \mathrm{~min}$ during total reaction time of $150 \mathrm{~min}$. The TOC concentration of the samples was monitored by a TOC analyzer (Apollo 9000, Teledyne Tekmar, USA). The UV/Visible spectrophotometer (Ultrospec 1100 pro) was used to determine the extinction coefficient of PEO.

\subsubsection{Preliminary Studies}

In order to determine the critical range for experimental design, different sets of experiments were carried out. Six different concentrations of PEO (10, 20, 30, 40, and $\left.50 \mathrm{mg} \mathrm{L}^{-1}\right)$ with 
different dosage of $\mathrm{H}_{2} \mathrm{O}_{2}\left(0,300,500,700,900\right.$, and $\left.1100 \mathrm{mg} \mathrm{L}^{-1}\right)$ were examined to study the percent of TOC removal. The results are presented in Figure 4.5 to Figure 4.9. As shown in these figures, in the absence of $\mathrm{H}_{2} \mathrm{O}_{2}$, TOC removal was not observed in all the cases. By contrast different experimental runs using UV combined with $\mathrm{H}_{2} \mathrm{O}_{2}$ result in a high percent of TOC removal (up to 100\%) in the studied range. Also, as illustrated in these figures, there is an optimum dosage for $\mathrm{H}_{2} \mathrm{O}_{2}$ above which the trend of TOC removal reverses. After the optimal point, higher $\mathrm{H}_{2} \mathrm{O}_{2}$ dosage reduces the process efficiency because excess $\mathrm{H}_{2} \mathrm{O}_{2}$ concentration reacts with hydroxyl radicals and produce hydroperoxyl radicals with lower oxidation potential. The reaction between hydroxyl radicals and hydrogen peroxide reduces the possibility of attacking hydroxyl radicals to the organic molecules that leads to a lower TOC removal efficiency. Therefore, the critical range of the operating conditions for the experimental design was determined based on the results of the preliminary studies.

\subsubsection{Experimental Design using Central Composite Design}

A four-factor and a five-level CCD combined with RSM along with quadratic programming were used to maximize the percent TOC removal as the process response (Ghafoori et al., 2012b). The CCD is considered as the most successful factorial design. In general, the quadratic programming is a linearly constrained optimization problem with a quadratic objective function. The initial PEO concentration $\left(X_{1}\right)$, the initial $\mathrm{H}_{2} \mathrm{O}_{2}$ dosage $\left(X_{2}\right)$, $\mathrm{pH}\left(X_{3}\right)$, and the recirculation rate $\left(X_{4}\right)$ were the four independent variables studied. The percent TOC removal was considered as the dependent factor (process response). The variables were coded at five levels between -2 and +2 . The critical ranges of the factors were selected based on a set of preliminary experiments. The ranges of the levels are shown in Table 4.2. 


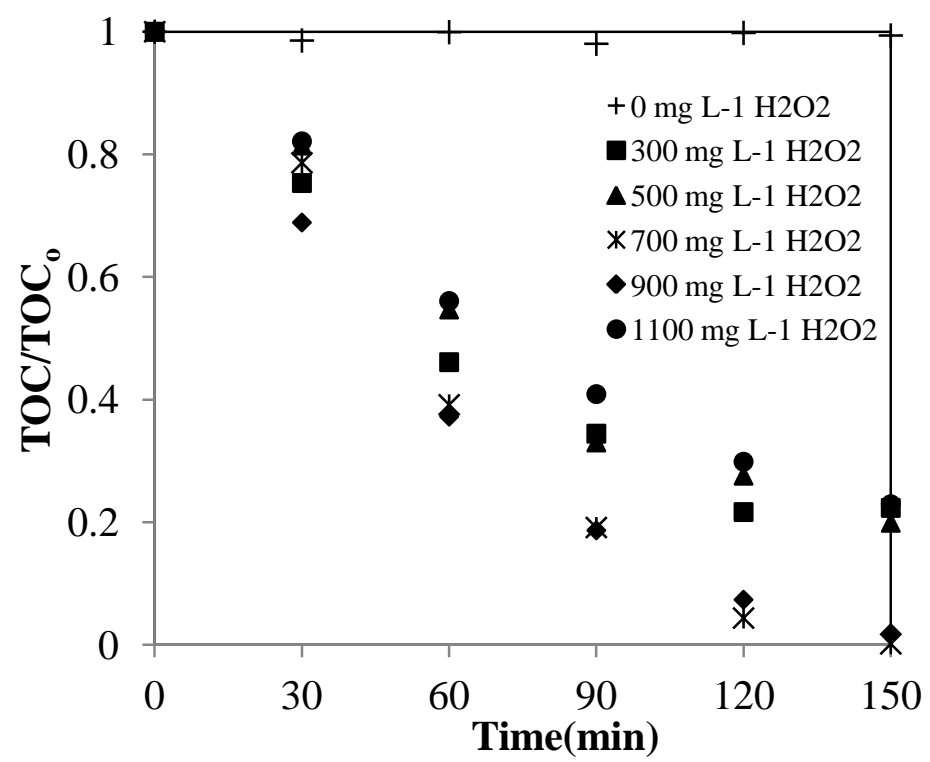

Figure 4.5. TOC removal of PEO solution by $\mathrm{UV} / \mathrm{H}_{2} \mathrm{O}_{2}$ process $\left([\mathrm{PEO}]_{0}=10 \mathrm{mg} \mathrm{L}^{-1}\right.$ ).

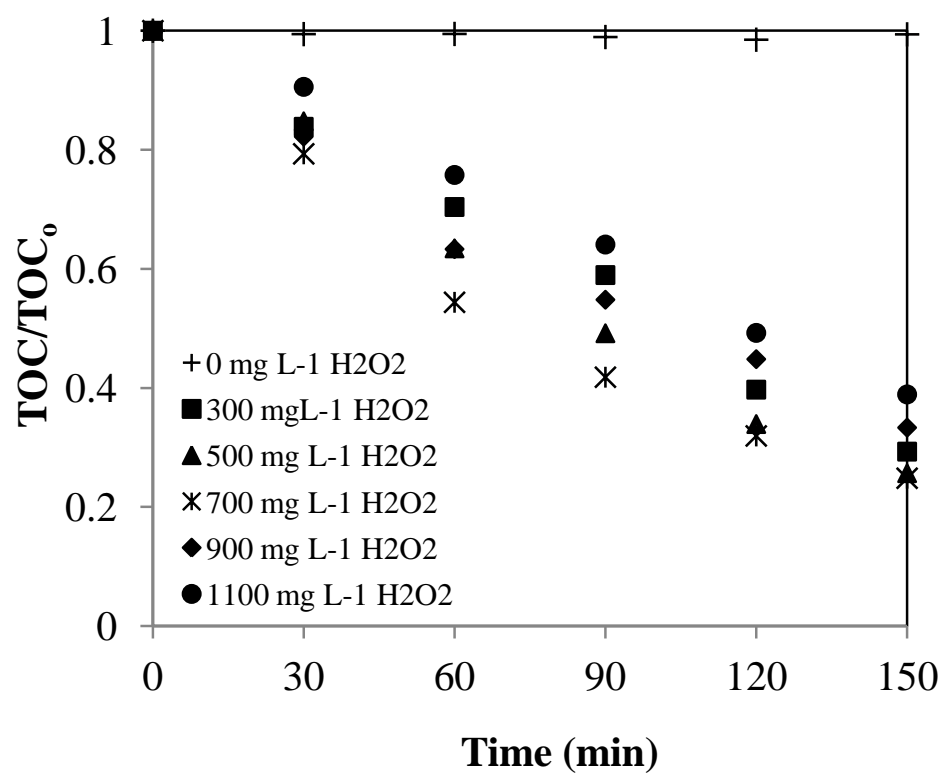

Figure 4.6. TOC removal of $\mathrm{PEO}$ solution by $\mathrm{UV} / \mathrm{H}_{2} \mathrm{O}_{2}$ process $\left([\mathrm{PEO}]_{0}=20 \mathrm{mg} \mathrm{L} \mathrm{L}^{-1}\right.$ ). 


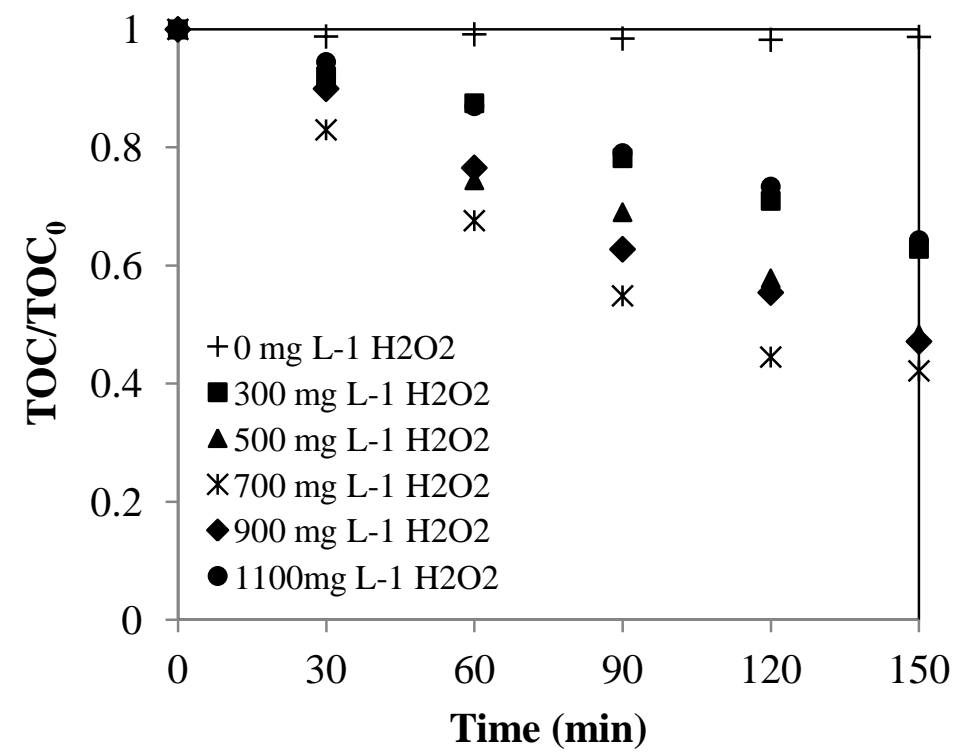

Figure 4.7. TOC removal of PEO solution by $\mathrm{UV} / \mathrm{H}_{2} \mathrm{O}_{2}$ process $\left([\mathrm{PEO}]_{0}=30 \mathrm{mg} \mathrm{L}^{-1}\right.$ ).

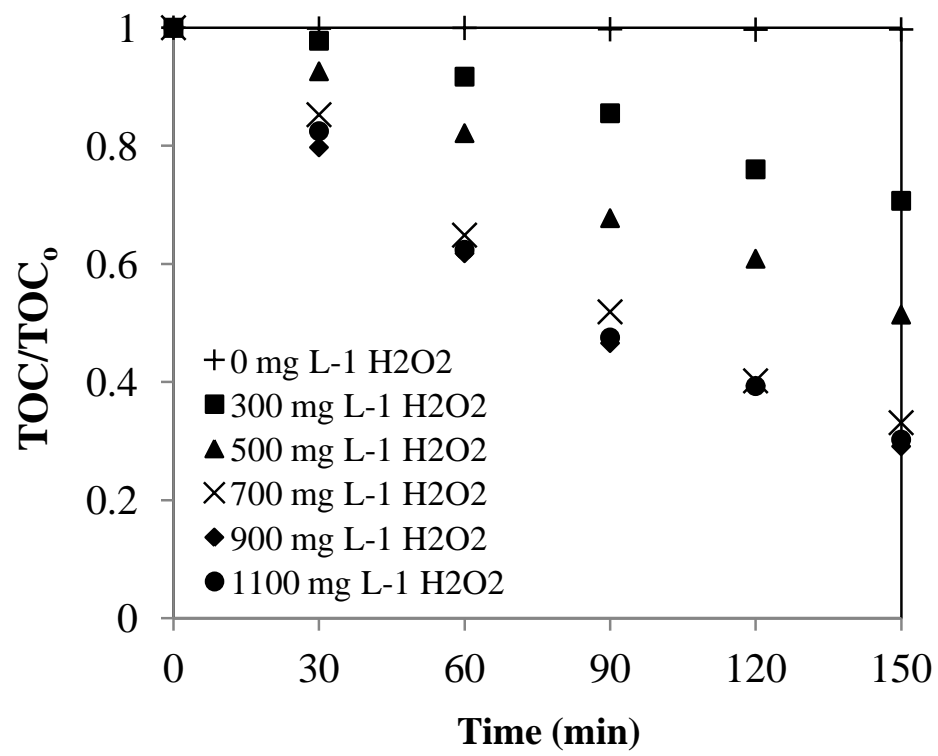

Figure 4.8. TOC removal of $\mathrm{PEO}$ solution by $\mathrm{UV} / \mathrm{H}_{2} \mathrm{O}_{2}$ process $\left([\mathrm{PEO}]_{0}=40 \mathrm{mg} \mathrm{\textrm {L } ^ { - 1 }}\right.$ ). 


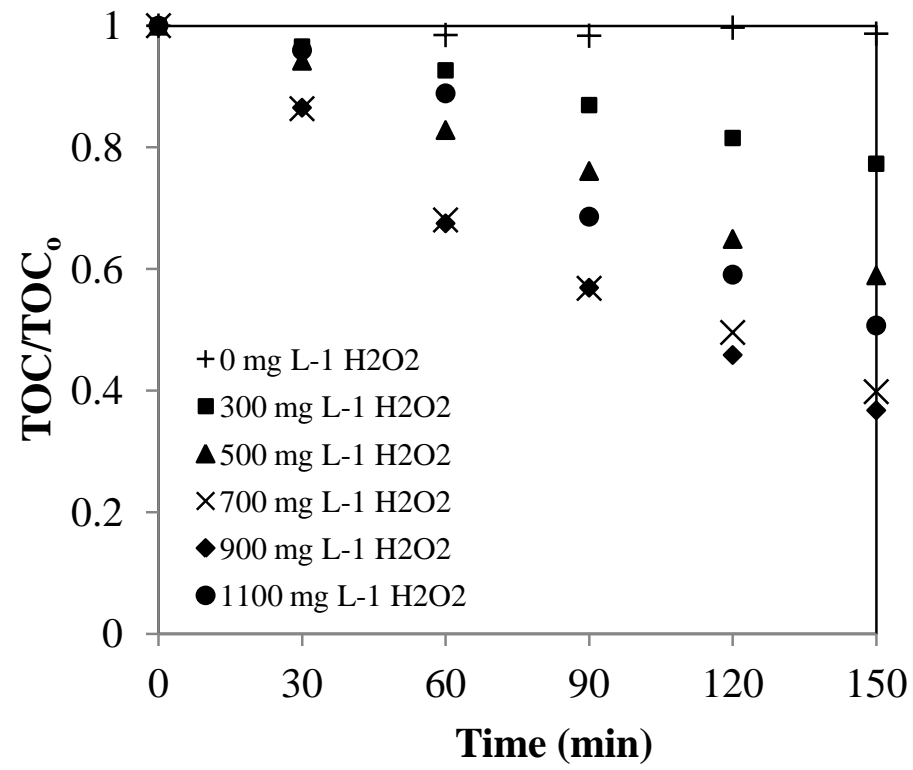

Figure 4.9. $\mathrm{TOC}$ removal of $\mathrm{PEO}$ solution by $\mathrm{UV} / \mathrm{H}_{2} \mathrm{O}_{2}$ process $\left([\mathrm{PEO}]_{0}=50 \mathrm{mg} \mathrm{L}^{-1}\right)$. 
Table 4.2. Independent variables and their coded levels for $\mathrm{UV} / \mathrm{H}_{2} \mathrm{O}_{2}$ process.

\begin{tabular}{|c|c|c|c|c|c|c|}
\hline \multirow[t]{2}{*}{ Independent Variables } & \multirow[t]{2}{*}{ Symbols } & \multicolumn{5}{|c|}{ Coded Levels } \\
\hline & & -2 & -1 & 0 & 1 & 2 \\
\hline Initial PEO concentration $\left(\mathrm{mg} \mathrm{L}^{-1}\right)$ & $X_{1}$ & 10 & 20 & 30 & 40 & 50 \\
\hline Initial $\mathrm{H}_{2} \mathrm{O}_{2}$ dosage $\left(\mathrm{mg} \mathrm{L}^{-1}\right)$ & $X_{2}$ & 300 & 500 & 700 & 900 & 1100 \\
\hline $\mathrm{pH}$ & $X_{3}$ & 3 & 5 & 7 & 9 & 11 \\
\hline Recirculation rate $\left(\mathrm{L} \min ^{-1}\right)$ & $X_{4}$ & 0.5 & 2.5 & 4.5 & 6.5 & 8.5 \\
\hline
\end{tabular}

A statistical software, Design-Expert 8.0.5 (trial version), was used for the regression analysis and the coefficient estimation of the response function. The statistical significance of the model equation was analyzed by the analysis of variance (ANOVA). The three-dimensional (3D) surface and the two-dimensional (2D) contour plots were developed while holding a variable constant in the quadratic model. The experimental and predicted values were compared to validate the model. The optimal operating conditions to maximize the percent TOC removal were also determined using a numerical technique built in the software.

Table 4.3 depicts the four-factor with five-level CCD and the observed and predicted values for the percent TOC removal by the developed quadratic model. As mentioned earlier, the RSM was used to estimate the parameters indicating an empirical relationship between input variables and the response. The quadratic model equation for predicting the response function (\%TOC removal) was expressed by the following second-order polynomial equation in terms of the coded factors $(k=4)$ :

$$
\begin{aligned}
& Y=63.33-2.95 X_{1}+1.53 X_{2}-1.03 X_{3}-0.15 X_{4}+0.06 X_{1} X_{2}-0.16 X_{1} X_{3}+0.27 X_{1} X_{4}- \\
& 0.14 X_{2} X_{3}+0.084 X_{2} X_{4}+0.075 X_{3} X_{4}+X_{1}^{2}-1.87 X_{2}{ }^{2}+0.99 X_{3}{ }^{2}+0.70 X_{4}{ }^{2}
\end{aligned}
$$




\subsubsection{Statistical Analysis}

The quadratic model equation can be recalculated considering only the significant factors. The statistical significance of the second-order polynomial model to predict the percent TOC removal was tested by the analysis of variance (ANOVA). The results of the ANOVA are presented in Table 4.4. The significance of each coefficient in Equation (4.28) was determined by the Fisher's $F$-test and values of probability $>F$. As shown in Table 4.4 , a small probability value $(p<0.0001)$ indicates that the model was highly significant. The goodness of fit of the model was validated by determination coefficient $\left(R^{2}\right)$. High $R^{2}$ values show the high significance of the model indicating the reliability of the second-order polynomial equation. Also, the adequate precision greater than 4 (16.48 in this case) shows that the model could be used to navigate the design space defined by the CCD. Adequate precision is a measure of the range in predicted response relative to its associated error, i.e., a signal to noise ratio. The normality of the data could be checked through the normal probability plot of the residuals. If the points on the plot lie on a straight line, the residuals are normally distributed as confirmed in Figure 4.10. Also, a high correlation between observed and predicted data shown in Figure 4.11 indicates their low discrepancies.

\subsubsection{Interaction Effects between the Independent Variables}

In order to study the interaction effects between the variables (the initial polymer concentration, the initial $\mathrm{H}_{2} \mathrm{O}_{2}$ dosage, $\mathrm{pH}$, and the recirculation rate), 3D response surface and 2D contour curves based on the quadratic model were plotted as shown in Figure 4.12 to Figure 4.17. As illustrated in those figures, the percent TOC removal was significantly affected by the initial polymer concentration and the initial $\mathrm{H}_{2} \mathrm{O}_{2}$ dosage. It could be seen that the percent TOC removal decreases by increasing the initial polymer concentration within the experimental range. 
Table 4.3. Four-factor Five-level CCD for RSM and the observed and predicted responses.

\begin{tabular}{|c|c|c|c|c|c|c|}
\hline \multirow[t]{2}{*}{ Run } & \multicolumn{4}{|c|}{ Independent Coded Variables } & \multicolumn{2}{|c|}{ TOC Removal (\%) } \\
\hline & $X_{1}$ & $X_{2}$ & $X_{3}$ & $X_{4}$ & Observed & Predicted \\
\hline 1 & -1 & -1 & -1 & -1 & 66.95 & 66.94 \\
\hline 2 & 1 & -1 & -1 & -1 & 60.01 & 60.70 \\
\hline 3 & -1 & 1 & -1 & -1 & 69.96 & 69.99 \\
\hline 4 & 1 & 1 & -1 & -1 & 65.99 & 63.99 \\
\hline 5 & -1 & -1 & 1 & -1 & 64.21 & 61.18 \\
\hline 6 & 1 & -1 & 1 & -1 & 59.48 & 58.44 \\
\hline 7 & -1 & 1 & 1 & -1 & 68.22 & 67.82 \\
\hline 8 & 1 & 1 & 1 & -1 & 61.68 & 61.18 \\
\hline 9 & -1 & -1 & -1 & 1 & 66.29 & 65.78 \\
\hline 10 & 1 & -1 & -1 & 1 & 61.50 & 60.62 \\
\hline 11 & -1 & 1 & -1 & 1 & 69.40 & 69.17 \\
\hline 12 & 1 & 1 & -1 & 1 & 66.36 & 64.25 \\
\hline 13 & -1 & -1 & 1 & 1 & 63.75 & 64.47 \\
\hline 14 & 1 & -1 & 1 & 1 & 59.68 & 58.67 \\
\hline 15 & -1 & 1 & 1 & 1 & 69.01 & 67.30 \\
\hline 16 & 1 & 1 & 1 & 1 & 62.99 & 61.74 \\
\hline 17 & -2 & 0 & 0 & 0 & 73.83 & 73.21 \\
\hline 18 & 2 & 0 & 0 & 0 & 58.51 & 61.43 \\
\hline 19 & 0 & -2 & 0 & 0 & 53.49 & 52.79 \\
\hline 20 & 0 & 2 & 0 & 0 & 55.95 & 58.91 \\
\hline 21 & 0 & 0 & -2 & 0 & 67.95 & 69.35 \\
\hline 22 & 0 & 0 & 2 & 0 & 64.34 & 65.23 \\
\hline 23 & 0 & 0 & 0 & -2 & 66.50 & 66.43 \\
\hline 24 & 0 & 0 & 0 & 2 & 63.50 & 65.83 \\
\hline 25 & 0 & 0 & 0 & 0 & 63.28 & 63.33 \\
\hline 26 & 0 & 0 & 0 & 0 & 62.60 & 63.33 \\
\hline 27 & 0 & 0 & 0 & 0 & 61.80 & 63.33 \\
\hline 28 & 0 & 0 & 0 & 0 & 63.18 & 63.33 \\
\hline 29 & 0 & 0 & 0 & 0 & 64.71 & 63.33 \\
\hline 30 & 0 & 0 & 0 & 0 & 64.19 & 63.33 \\
\hline 31 & 0 & 0 & 0 & 0 & 63.52 & 63.33 \\
\hline
\end{tabular}

The effect of the initial PEO concentration on the percent TOC removal is mainly due to the absorption of the UV radiation by PEO molecules. An increase in the initial PEO concentration leads to induce an inner filter effect which indicates the reabsorption by the PEO molecule (Xu et al., 2009). Therefore, the permeability of the UV light becomes less at higher concentration of PEO as it can be also verified later in the absorption model that higher concentration of PEO results in higher UV absorption by PEO. This results in decreasing the UV radiation absorption by $\mathrm{H}_{2} \mathrm{O}_{2}$ and subsequently less formation of hydroxyl radicals which are the major cause of the 
PEO decomposition. Increasing the initial $\mathrm{H}_{2} \mathrm{O}_{2}$ dosage results in enhancing the percent TOC removal up to an optimum $\mathrm{H}_{2} \mathrm{O}_{2}$ dosage, where the trend reverses after this point. This is due to the fact that the excess $\mathrm{H}_{2} \mathrm{O}_{2}$ scavenges hydroxyl radicals (Mehrvar et al., 2001; Kralik et al., 2010). Also, it is obvious that the percent TOC removal decreases at higher $\mathrm{pH}$ where the maximum percent TOC removal is achieved at the lower initial $\mathrm{pH}$. In alkaline solutions, the dissociated form of hydrogen peroxide $\left(\mathrm{HO}_{2}^{-}\right)$reacts with hydroxyl radicals more than two orders of magnitude faster than hydrogen peroxide (Reaction (R2) and (R3) as shown in Table 4.1). Therefore, the oxidation efficiency decreases as hydroxyl radicals are consumed. However, as it is evident in Figure 4.12 to Figure 4.17, the influence of the $\mathrm{pH}$ on the percent TOC removal is less pronounced than the initial polymer concentration and the initial $\mathrm{H}_{2} \mathrm{O}_{2}$ dosage.

Table 4.4. ANOVA for prediction of percent TOC removal by the quadratic model (CCD).

\begin{tabular}{|c|c|c|c|c|c|c|}
\hline \multirow{2}{*}{$\begin{array}{l}\text { Factors } \\
\text { (coded) }\end{array}$} & \multicolumn{6}{|l|}{ Statistics } \\
\hline & Sum of Squares & $d f^{1}$ & Mean Square & $F$-Value & $\begin{array}{l}p \text {-Value } \\
\text { Prob }>F\end{array}$ & Remark \\
\hline Model & 481.14 & 14 & 34.37 & 10.85 & $<0.0001^{2}$ & Significant \\
\hline$X_{1}$ & 208.51 & 1 & 208.51 & 65.84 & $<0.0001$ & Significant \\
\hline$X_{2}$ & 56.00 & 1 & 56.00 & 17.68 & 0.0007 & Significant \\
\hline$X_{3}$ & 25.34 & 1 & 25.34 & 8.00 & 0.0121 & Significant \\
\hline$X_{4}$ & 0.52 & 1 & 0.52 & 0.16 & 0.6917 & \\
\hline$X_{1} X_{2}$ & 0.058 & 1 & 0.058 & 0.018 & 0.8944 & \\
\hline$X_{1} X_{3}$ & 0.43 & 1 & 0.43 & 0.14 & 0.7176 & \\
\hline$X_{1} X_{4}$ & 1.13 & 1 & 1.13 & 0.36 & 0.5579 & \\
\hline$X_{2} X_{3}$ & 0.30 & 1 & 0.30 & 0.094 & 0.7634 & \\
\hline$X_{1} X_{4}$ & 0.11 & 1 & 0.11 & 0.035 & 0.8530 & \\
\hline$X_{3} X_{4}$ & 0.090 & 1 & 0.090 & 0.028 & 0.8682 & \\
\hline$X_{1}^{2}$ & 28.36 & 1 & 28.36 & 8.96 & 0.0086 & Significant \\
\hline$X_{2}{ }^{2}$ & 99.64 & 1 & 99.64 & 31.46 & $<0.0001$ & Significant \\
\hline$X_{3}^{2}$ & 28.00 & 1 & 28.00 & 8.84 & 0.0090 & Significant \\
\hline$X_{4}^{2}$ & 14.15 & 1 & 14.15 & 4.47 & 0.0506 & \\
\hline Pure Error & 5.58 & 6 & 0.93 & & & \\
\hline Cor Total & 531.81 & 30 & & & & \\
\hline
\end{tabular}


Also, as shown in Table 4.4 and Figure 4.14, Figure 4.16, and Figure 4.17 the recirculation rate does not have any significant effect on the percent TOC removal. This is also confirmed in Table 4.4 showing the significance of the factors and their interaction.

\subsubsection{Optimization of the Process Parameters}

The RSM was used to determine the values of the four independent variables that result in a maximum percent TOC removal. The optimization of experimental conditions was carried out by maximizing the percent TOC removal at defined optimization criteria for the factors (Table 4.2). Therefore, the optimum operating conditions were found using the numerical technique built in the Design Expert Software 8.0.5 according to the developed model (Equation (4.28)). The numerical optimization searches the design space using the developed model in the analysis to find factor settings that meet the goal of maximizing TOC removal. The optimum values to achieve the maximum $84 \%$ TOC removal after $150 \mathrm{~min}$ were $10 \mathrm{mg} \mathrm{L}^{-1} \mathrm{PEO}, 780 \mathrm{mg} \mathrm{L}^{-1} \mathrm{H}_{2} \mathrm{O}_{2}$, $\mathrm{pH} 3$, and $0.5 \mathrm{~L} \mathrm{~min}^{-1}$ recirculation rate. The TOC removal efficiency was obtained experimentally at the optimum operating conditions to validate the model prediction. $82 \%$ TOC removal achieved experimentally at the optimal operating conditions confirmed the results of the developed model. 


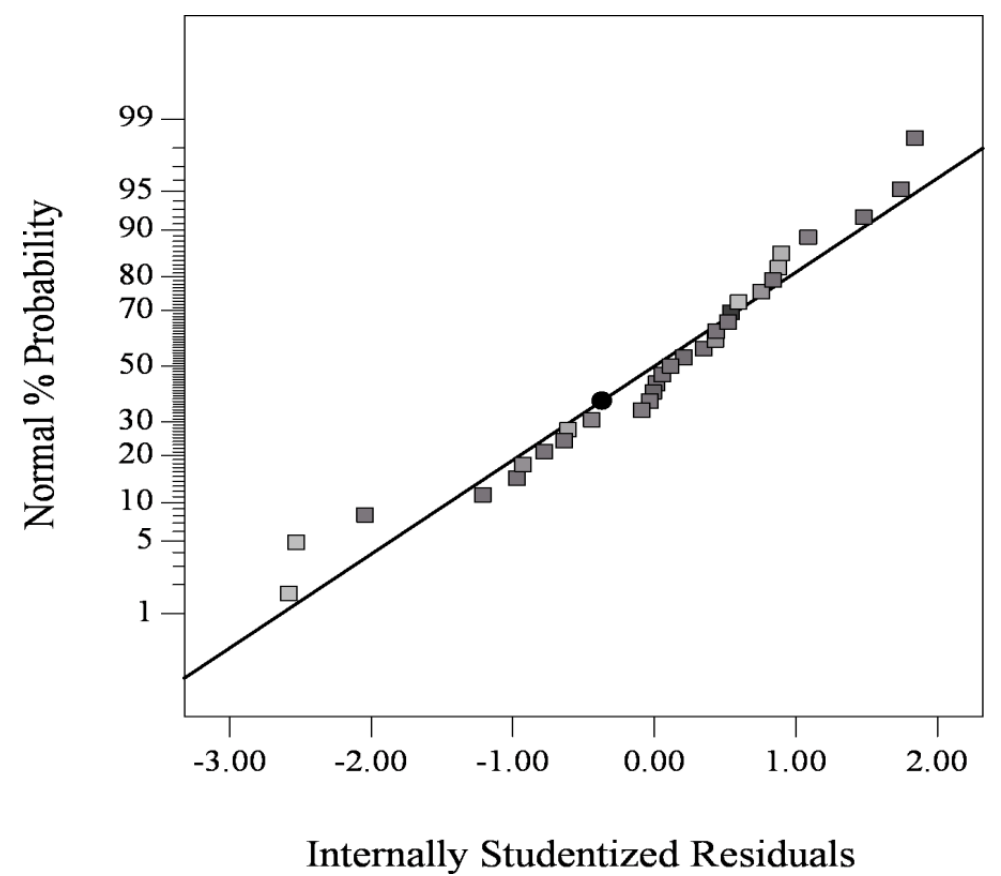

Figure 4.10. Normal probability plot of the internally studentized residuals.

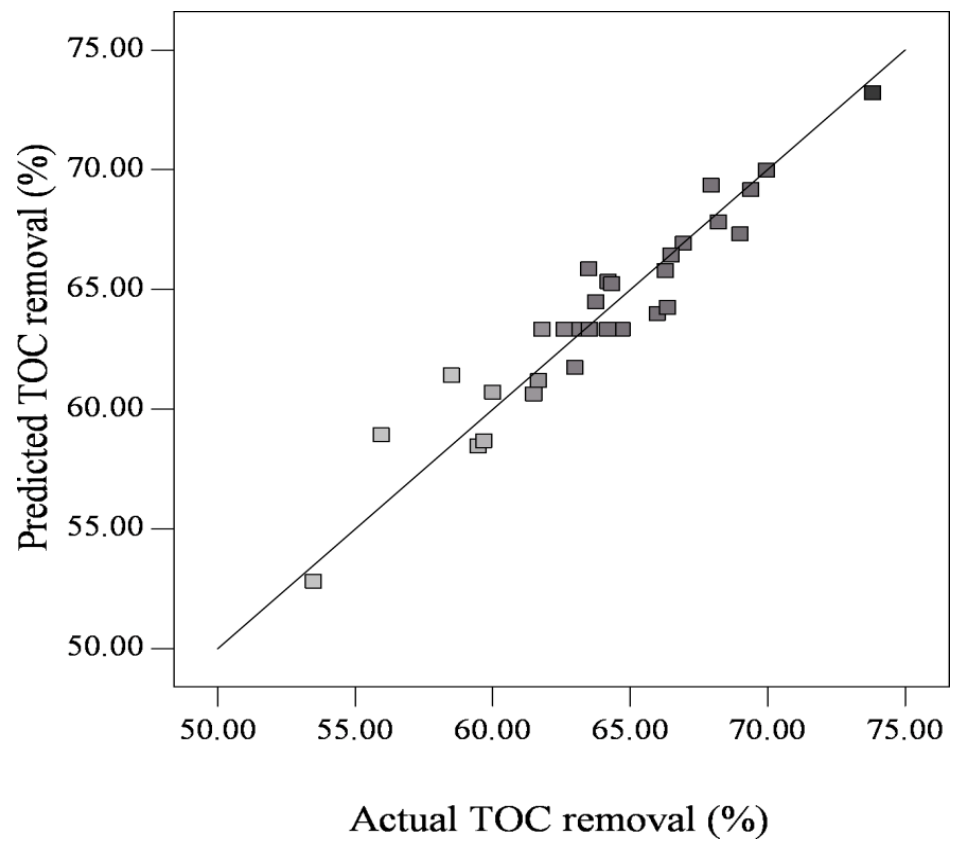

Figure 4.11. Comparison of predicted percent TOC removal versus actual values. 


\subsubsection{Kinetic Model Development and Photoreactor Modeling}

The reaction scheme for the photodegradation of PEO by the $\mathrm{UV} / \mathrm{H}_{2} \mathrm{O}_{2}$ process is summarized in Table 4.5. All reaction mechanisms are based on extensively accepted photochemical reactions and the rate constants in advanced oxidation processes (Reactions (R1) to (R13)). Complete mineralization of PEO monomer to $\mathrm{H}_{2} \mathrm{O}$ and $\mathrm{CO}_{2}$ disregarding other intermediates is presented in Reactions (R14) to (R16). The reaction rates for the last three reactions are known and will be determined through the kinetic parameter estimation. A kinetic model based on the mass balance of species of interest in reaction steps proposed in Table 4.5 was developed. The kinetic model predicts the TOC concentration profile of the polymer as a function of time. The model assumptions are as follows:

- The $\mathrm{pH}$ is considered constant as it is proven that $\mathrm{pH}$ changes during the process has no significant effect on the model predictions (Crittenden et al., 1999);

- The photon absorption by PEO does not show any TOC removal indicating that the quantum yield in direct photolysis of PEO (PEO $\stackrel{h v, \varphi_{T O C}}{\longrightarrow}$ ?) is zero, i.e., $\varphi_{T O C}=0$;

- Only hydroxyl radical attack is mainly responsible for the polymer degradation as the oxidation potential of other radicals are negligible compared to that of hydroxyl radicals;

- $\mathrm{H}_{2} \mathrm{O}_{2}$ and $\mathrm{HO}_{2}^{-}$, acid-base conjugates, are the main light-absorbing species;

- Oxidation intermediates of PEO other than ethylene oxide (monomer) are not considered in the rate expressions; and

- Unlike most kinetic modeling approaches, unsteady state assumptions are used in the free radical rate expressions to have a better prediction of the degradation in the completelymixed recirculation batch system. 


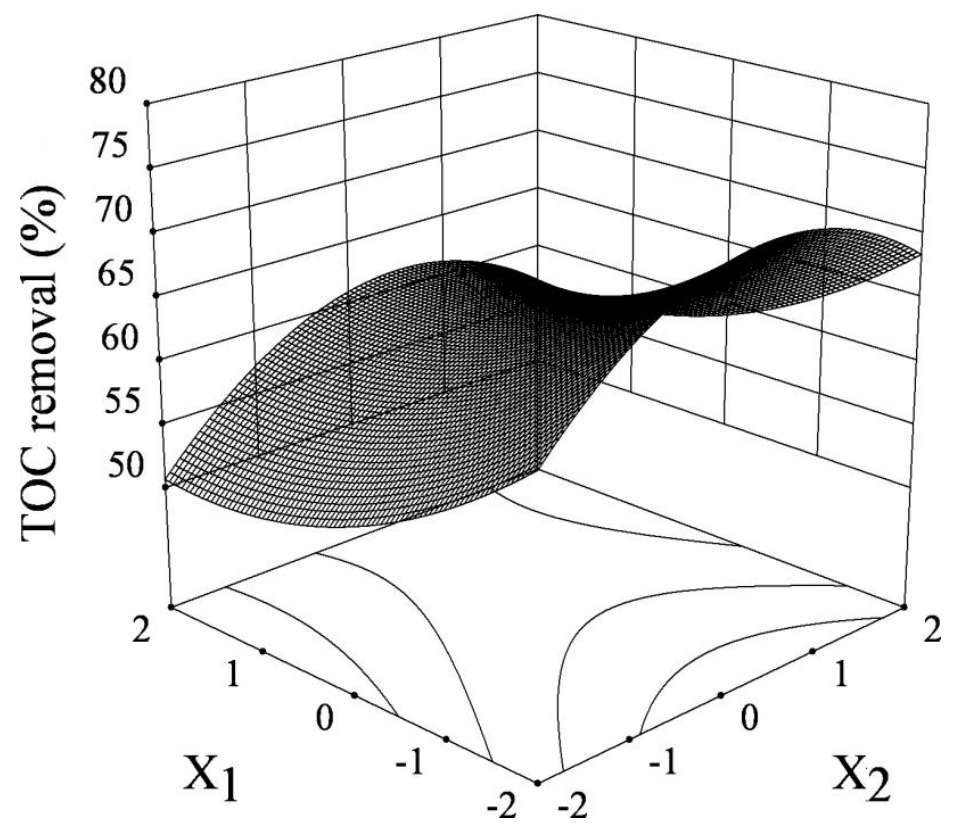

Figure 4.12. The effects of PEO concentration $\left(X_{1}\right)$ and $\mathrm{H}_{2} \mathrm{O}_{2}$ dosage $\left(X_{2}\right)$ on percent TOC removal.

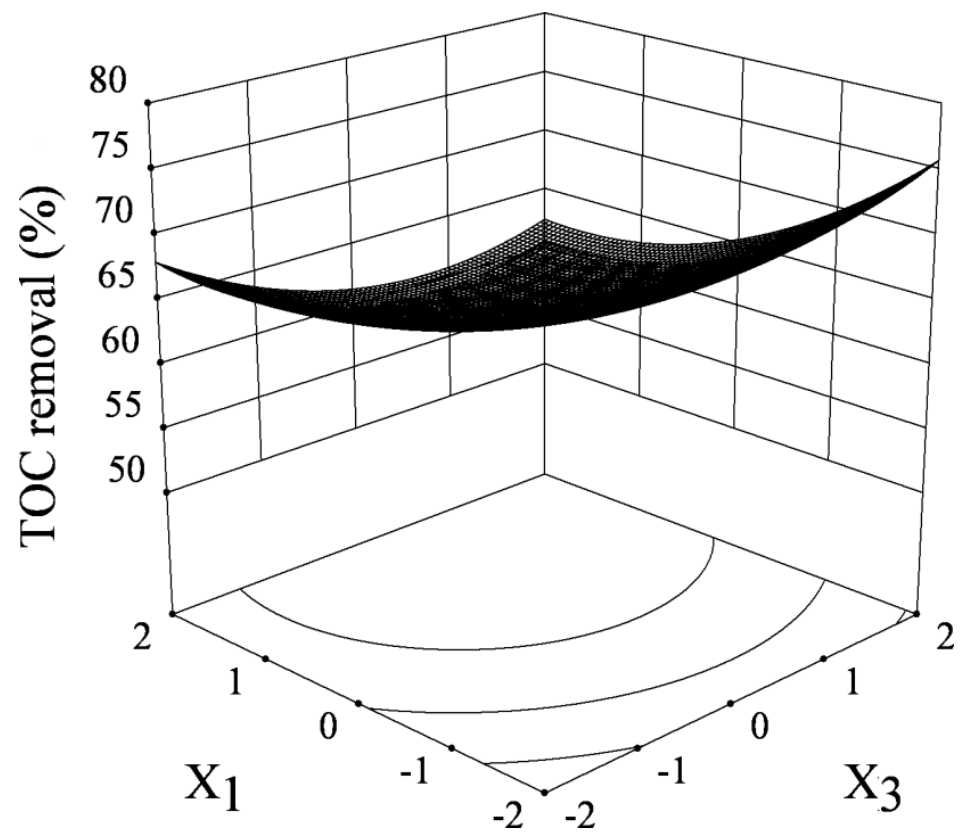

Figure 4.13. The effects of PEO concentration $\left(X_{1}\right)$ and $\mathrm{pH}\left(X_{3}\right)$ on Percent TOC removal. 


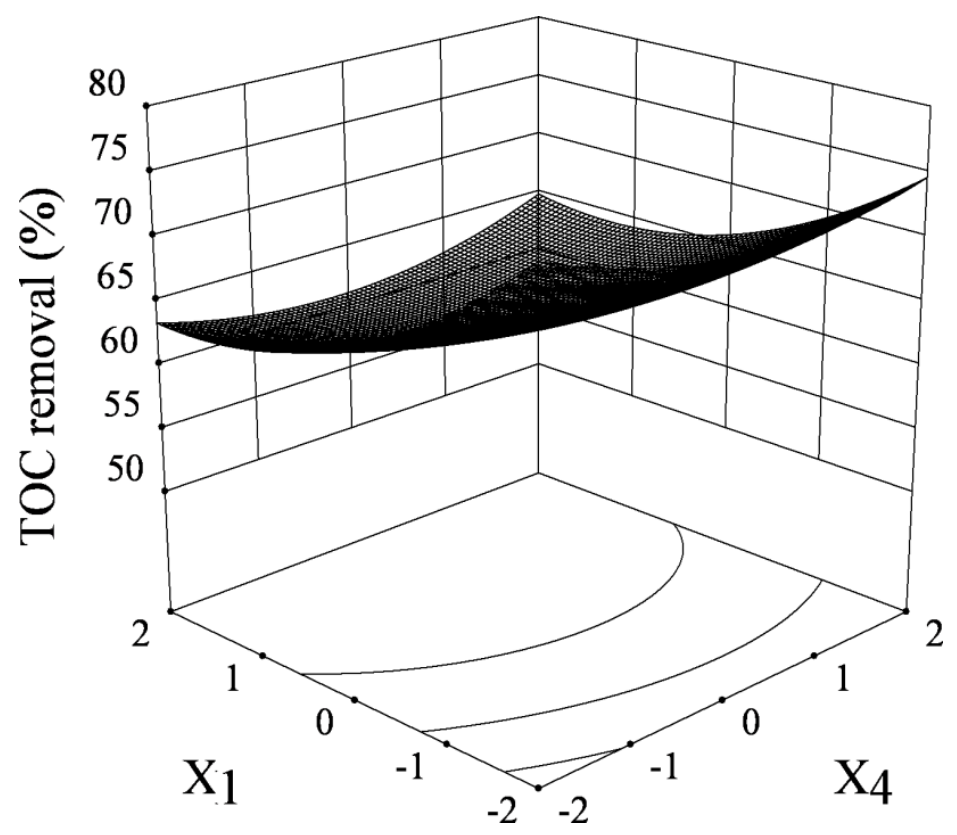

Figure 4.14. The effects of PEO concentration $\left(X_{1}\right)$ and recirculation rate $\left(X_{4}\right)$ on percent TOC removal.

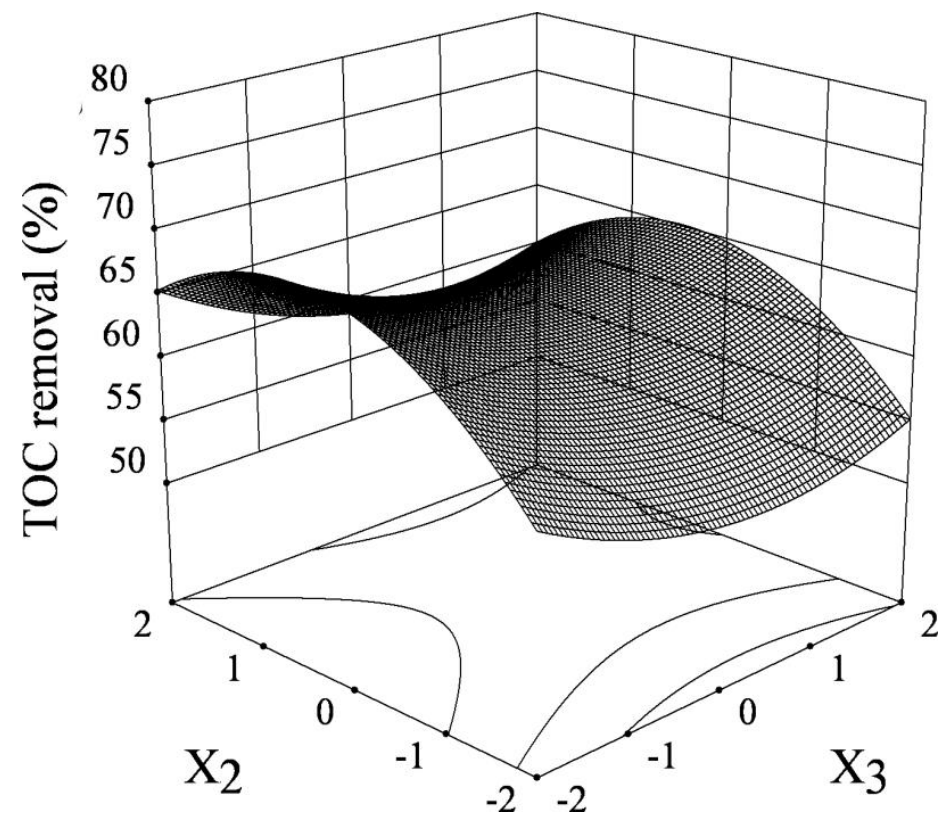

Figure 4.15. The effects of $\mathrm{H}_{2} \mathrm{O}_{2}$ concentration $\left(X_{2}\right)$ and $\mathrm{pH}\left(X_{3}\right)$ on percent TOC removal. 


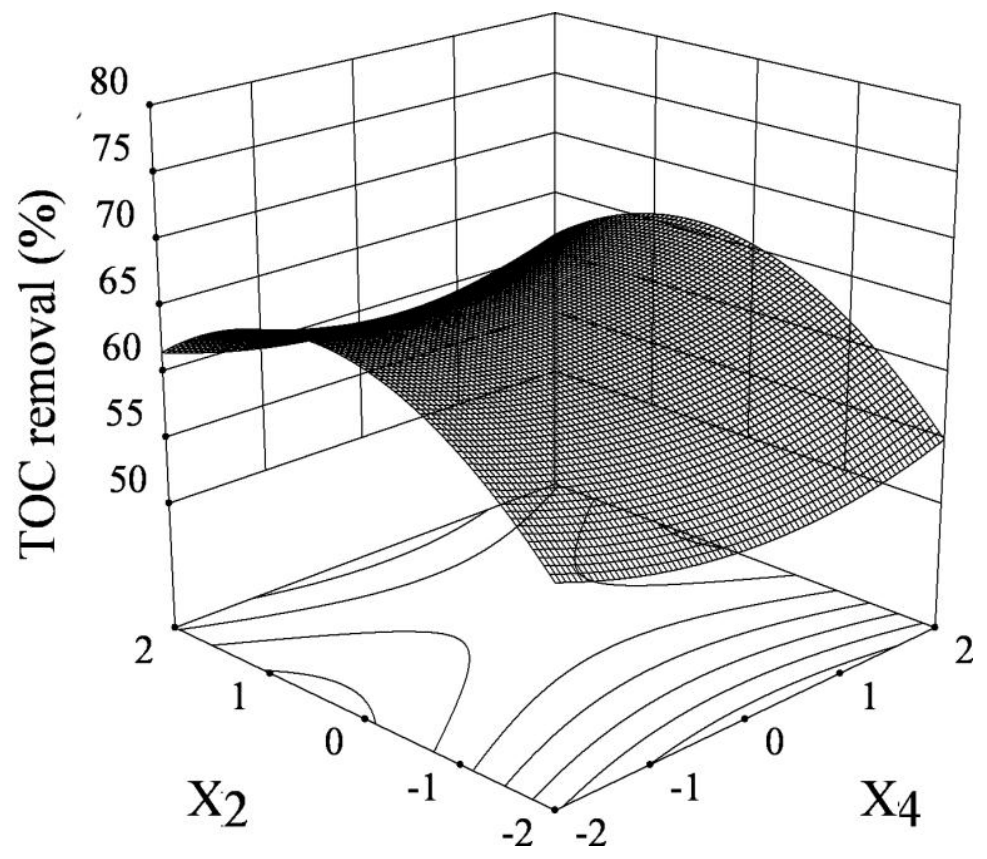

Figure 4.16. The effects of $\mathrm{H}_{2} \mathrm{O}_{2}$ concentration $\left(X_{2}\right)$ and recirculation rate $\left(X_{4}\right)$ on percent TOC removal.

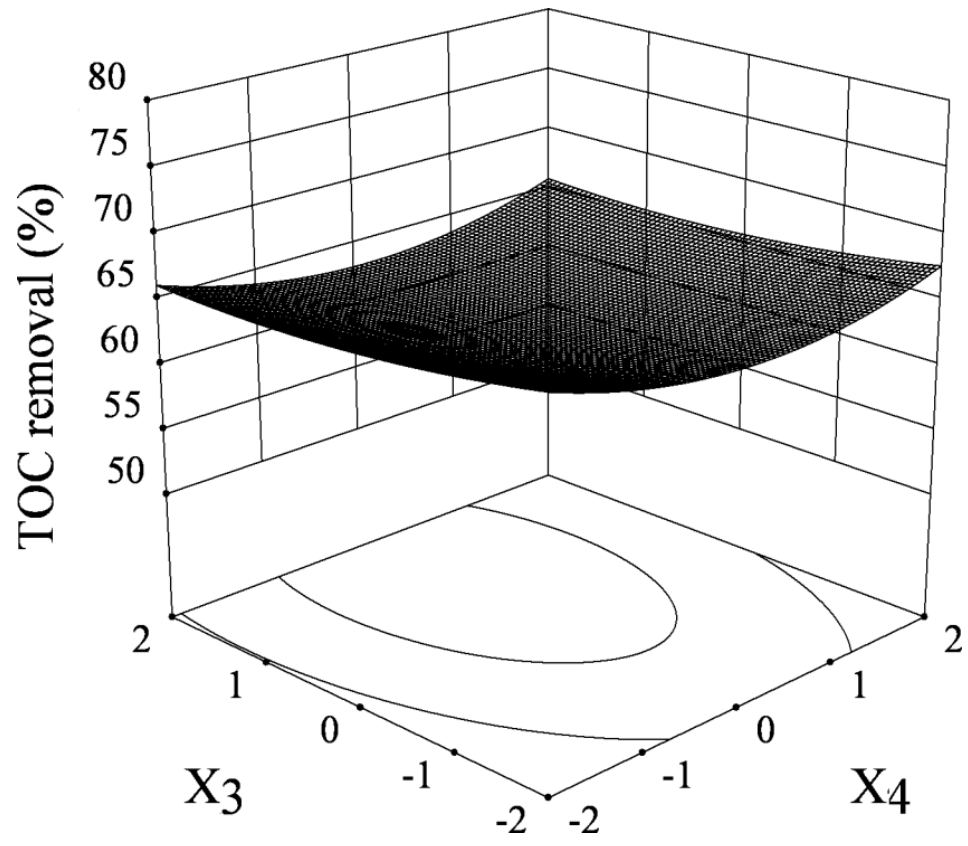

Figure 4.17. The effects of $\mathrm{pH}\left(X_{3}\right)$ and recirculation rate $\left(X_{4}\right)$ on percent TOC removal. 
Table 4.5. Reaction mechanism for complete mineralization of PEO by $\mathrm{UV} / \mathrm{H}_{2} \mathrm{O}_{2}$.

\begin{tabular}{|c|c|c|c|}
\hline No. & Reaction & Rate Constant & References \\
\hline R1 & $\mathrm{H}_{2} \mathrm{O}_{2} \stackrel{\varphi_{\mathrm{H}_{2} \mathrm{O}_{2}}, h v}{\longrightarrow} 2 \cdot \mathrm{OH}$ & $0.5\left(\right.$ mol Einstein $\left.^{-1}\right)$ & Crittenden et al., 1999 \\
\hline R2 & $\cdot \mathrm{OH}+\mathrm{H}_{2} \mathrm{O}_{2} \stackrel{k_{2}}{\rightarrow} \mathrm{HO}_{2}^{\cdot}+\mathrm{H}_{2} \mathrm{O}$ & $2.7 \times 10^{7} \quad\left(\mathrm{M}^{-1} \mathrm{~s}^{-1}\right)$ & Buxton et al., 1988 \\
\hline R3 & $\cdot \mathrm{OH}+\mathrm{HO}_{2}^{-} \stackrel{k_{3}}{\rightarrow} \mathrm{HO}_{2}^{\cdot}+\mathrm{OH}^{-}$ & $7.5 \times 10^{9} \quad\left(\mathrm{M}^{-1} \mathrm{~s}^{-1}\right)$ & Christensen et al., 1982 \\
\hline R4 & $\mathrm{HO}_{2}+\mathrm{H}_{2} \mathrm{O}_{2} \stackrel{k_{4}}{\rightarrow} \cdot \mathrm{OH}+\mathrm{H}_{2} \mathrm{O}+\mathrm{O}_{2}$ & $\left(\mathrm{M}^{-1} \mathrm{~s}^{-1}\right)$ & Koppenol et al., 1978 \\
\hline R5 & $\mathrm{O}_{2}^{\bullet-}+\mathrm{H}_{2} \mathrm{O}_{2} \stackrel{k_{5}}{\rightarrow} \cdot \mathrm{OH}+\mathrm{O}_{2}+\mathrm{OH}^{-}$ & $0.13 \quad\left(\mathrm{M}^{-1} \mathrm{~s}^{-1}\right)$ & Weinstein and Bielski, 1979 \\
\hline R6 & $\mathrm{O}_{2}^{\cdot-}+\mathrm{H}^{+} \stackrel{k_{6}}{\rightarrow} \mathrm{HO}_{2}^{\cdot}$ & $1 \times 10^{10} \quad\left(\mathrm{M}^{-1} \mathrm{~s}^{-1}\right)$ & Bielski et al., 1985 \\
\hline R7 & $\mathrm{HO}_{2} \stackrel{k_{7}}{\rightarrow} \mathrm{O}_{2}^{--}+H^{+}$ & $1.58 \times 10^{5}\left(\mathrm{~s}^{-1}\right)$ & Bielski et al., 1985 \\
\hline $\mathrm{R} 8$ & $\cdot \mathrm{OH}+\cdot \mathrm{OH}^{\stackrel{k_{8}}{\rightarrow}} \mathrm{H}_{2} \mathrm{O}_{2}$ & $5.5 \times 10^{9} \quad\left(\mathrm{M}^{-1} \mathrm{~s}^{-1}\right)$ & Buxton et al., 1988 \\
\hline R9 & $\mathrm{HO}_{2}^{\cdot}+\mathrm{HO}_{2}^{\cdot} \stackrel{k_{9}}{\rightarrow} \mathrm{H}_{2} \mathrm{O}_{2}+\mathrm{O}_{2}$ & $8.3 \times 10^{5} \quad\left(\mathrm{M}^{-1} \mathrm{~s}^{-1}\right)$ & Bielski et al., 1985 \\
\hline R10 & $\cdot \mathrm{OH}+\mathrm{HO}_{2}^{\cdot} \stackrel{k_{10}}{\longrightarrow} \mathrm{O}_{2}+\mathrm{H}_{2} \mathrm{O}$ & $6.6 \times 10^{9} \quad\left(\mathrm{M}^{-1} \mathrm{~s}^{-1}\right)$ & Elliot and Buxton, 1992 \\
\hline R11 & $\mathrm{HO}_{2}^{-}+\mathrm{O}_{2}^{--} \stackrel{k_{11}}{\longrightarrow} \mathrm{HO}_{2}^{-}+\mathrm{O}_{2}$ & $9.7 \times 10^{7} \quad\left(\mathrm{M}^{-1} \mathrm{~s}^{-1}\right)$ & Bielski et al., 1985 \\
\hline R12 & $\cdot \mathrm{OH}+\mathrm{O}_{2}^{--} \stackrel{k_{12}}{\rightarrow} \mathrm{O}_{2}+\mathrm{OH}^{-}$ & $8 \times 10^{9} \quad\left(\mathrm{M}^{-1} \mathrm{~s}^{-1}\right)$ & Linden et al., 2005 \\
\hline R13 & $\mathrm{H}_{2} \mathrm{O}_{2} \leftrightarrow H^{+}+\mathrm{HO}_{2}^{-}$ & $\mathrm{p} K_{a}=11.6$ & Perry et al., 1981 \\
\hline R14 & $P_{n}+{ }^{\bullet} O H \stackrel{k_{14}}{\longrightarrow} R_{n}^{\cdot}+H_{2} O$ & To be determined & This study \\
\hline R15 & $R_{n}^{\cdot} \stackrel{k_{15}}{\longrightarrow} \sum\left(R_{n}^{\cdot}-R_{n-1}^{\cdot}\right)+R_{1}^{\bullet}\left(M_{1}\right)$ & To be determined & This study \\
\hline R16 & $\mathrm{M}_{1}+\cdot \cdot \mathrm{OH}^{k_{16}} \rightarrow \ldots \rightarrow \mathrm{CO}_{2}+\mathrm{H}_{2} \mathrm{O}$ & To be determined & This study \\
\hline
\end{tabular}


As mentioned earlier, the kinetic modeling of the polymer photodegradation (Reactions (R14) to (R16)) in an aqueous solution needs more attention since the degradation of polydisperse high molecular weight materials results in the formation of thousands of polymeric chains with different chain lengths and various chemical compositions.

In this study, the chain-end scission (unzipping) is considered as the mechanism of the chain cleavage as it is usually the case in complete mineralization (Sterling and McCoy, 2001). Therefore, the reaction mechanism is based on the degradation of PEO to its monomer (ethylene oxide). The initiation step in the generalized mechanism is the photolysis of $\mathrm{H}_{2} \mathrm{O}_{2}$ that results in the formation of highly reactive hydroxyl radicals $\left({ }^{\circ} \mathrm{OH}\right)$ that attack the target compound (PEO) leading to the chain scission. To be more precise, the hydrogen abstraction of the polymer of the chain length $n\left(P_{n}\right)$ results in the generation of the chain-end radical $\left(R_{n}^{\bullet}\right)$ as shown in Reaction (R14). The $\beta$-scission of an end radical leads to the monomer formation $\left(M_{1}\right)$ according to the chain-end scission as presented in Reaction (R15). The monomer degradation results in the complete mineralization of the polymer to $\mathrm{H}_{2} \mathrm{O}$ and $\mathrm{CO}_{2}(\mathrm{R} 16)$ disregarding the intermediates formed during the course of reactions.

To overcome the difficulties of kinetic modeling of polymers, population balance equations (PBE) are employed. A free radical mechanism is proposed to evaluate rate coefficients based on the continuous-distribution kinetics which provides the details of the degradation process by taking into account the time dependency of the TOC concentration. As mentioned earlier, the chain-end scission is considered as the main mechanism of the photodegradation. According to the long chain approximation (LCA), initiation-termination steps are neglected because of the insignificant effect on the overall reaction rates (Nigam et al., 1994). As shown in Reaction (R14) in Table 4.5, the hydrogen abstraction by hydroxyl radicals from the polymer of the chain 
length $n$ results in the formation of radicals of the same chain length $\left(R_{n}^{*}\right)$. The binary degradation of $R_{n}^{\bullet}$ could be represented by the following sequential reactions:

$$
\begin{aligned}
& R_{n}^{\bullet} \rightarrow\left(R_{n}^{\bullet}-R_{n-1}^{\bullet}\right)+R_{n-1}^{\bullet} \\
& R_{n-1}^{\bullet} \rightarrow\left(R_{n-1}^{\bullet}-R_{n-2}^{\bullet}\right)+R_{n-2}^{\bullet} \\
& \vdots \\
& R_{2}^{\bullet} \rightarrow\left(R_{2}^{\bullet}-R_{1}^{\bullet}\right)+R_{1}^{\bullet}\left(M_{1}\right)
\end{aligned}
$$

which, in turn, could be written as follows:

$R_{n}^{\bullet} \rightarrow\left(R_{n}^{\bullet}-R_{n-1}^{\bullet}\right)+\left(R_{n-1}^{\bullet}-R_{n-2}^{\bullet}\right)+\cdots+\left(R_{2}^{\bullet}-R_{1}^{\bullet}\right)+R_{1}^{\bullet}\left(M_{1}\right)$

Generally, for a binary fragmentation reaction, the product kinetics is represented by Equation (3) in which the integral is used instead of the sum to make the calculations easier.

$R(n)=\int_{n}^{\infty} k\left(n^{\prime}\right) \Omega\left(n, n^{\prime}\right) r\left(n^{\prime}, t\right) \mathrm{d} n^{\prime}$

In this equation, $k\left(n^{\prime}\right)$ and $r\left(n^{\prime}, t\right)$ are the rate constant of the binary scission and the chain length distribution of the polymer radical, respectively. The stoichiometric coefficient $\Omega\left(n, n^{\prime}\right)$ represents the likelihood of obtaining a scission product of the chain length $n$ and $n^{\prime}-n$ from a larger molecule $n^{\prime}$. A general expression for the stoichiometric coefficient, or kernel, is given by the following expression:

$\Omega\left(n, n^{\prime}\right)=\frac{n^{m}\left(n^{\prime}-n\right)^{m} \Gamma(2 m+2)}{\Gamma(m+1)^{2}\left(n^{\prime}\right)^{2 m+1}}$

where $\Gamma$ is the gamma function and $m$ is a parameter indicating the shape of the scission fragment distribution. For a chain-end scission, when a specific product of a fixed chain length $l$ and a chain length of $n^{\prime}-1$ are formed, Dirac delta functions are used to represent the stoichiometric kernels, $\Omega\left(n^{\prime}-1, n^{\prime}\right)=\delta\left(n-\left(n^{\prime}-1\right)\right)$ and $\Omega\left(n, n^{\prime}\right)=\delta(n-1)$, respectively (Konaganti and Madras, 2009). The stoichiometric coefficient $\delta\left(n-\left(n^{\prime}-1\right)\right)$ ensures that a 
product has the chain length of $n=n^{\prime}-1$ and that $n^{\prime} \geq n$ is valid in the interval of integration. A mathematical model was developed in the form of a set of ordinary differential equations (ODEs) based on the reaction scheme presented in Table 4.5. The proposed model represents the kinetic expressions of the principle species in the system. The primary reaction that initiates the chain reaction is the direct photolysis of $\mathrm{H}_{2} \mathrm{O}_{2}$ to two hydroxyl radical molecules as shown below:

$R_{U V, H_{2} O_{2}}=\varphi_{H_{2} O_{2}} e_{\lambda}^{a}$

where $\varphi_{\mathrm{H}_{2} \mathrm{O}_{2}}$ is the quantum yield of $\mathrm{H}_{2} \mathrm{O}_{2}$ and $\mathrm{HO}_{2}^{-}$[mol Einstein ${ }^{-1}$ ] and $e_{\lambda}^{a}$ is the local volumetric rate of energy absorption (LVREA) [Einstein $\mathrm{L}^{-1} \mathrm{~s}^{-1}$ ].

$\mathrm{H}_{2} \mathrm{O}_{2}$ and $\mathrm{HO}_{2}^{-}$, acid-base conjugates, are interrelated through the equilibrium Reaction (R13). The total concentration of $\mathrm{H}_{2} \mathrm{O}_{2}$ at equilibrium is defined as follows (Ghafoori et al., 2012b):

$\left[\mathrm{H}_{2} \mathrm{O}_{2}\right]_{T}=\left[\mathrm{H}_{2} \mathrm{O}_{2}\right]+\left[\mathrm{HO}_{2}^{-}\right]$

Consequently, a new equilibrium concentration for the two individual species can be expressed as follows (Edalatmanesh et al., 2008b):

$$
\begin{aligned}
& {\left[\mathrm{H}_{2} \mathrm{O}_{2}\right]=\frac{\left[\mathrm{H}_{2} \mathrm{O}_{2}\right]_{T}}{1+\mathrm{K}_{a}\left[\mathrm{H}^{+}\right]^{-1}}} \\
& {\left[\mathrm{HO}_{2}^{-}\right]=\frac{\left[\mathrm{H}_{2} \mathrm{O}_{2}\right]_{T}}{1+\mathrm{K}_{a}^{-1}\left[\mathrm{H}^{+}\right]}}
\end{aligned}
$$

where $K_{a}$ is the equilibrium constant. These concentrations are used later in the developed kinetic model (Equations (4.48) to (4.42)). The LVREA is defined according to the BeerLambert law, which is based on the radiation balance (Ghafoori et al., 2012b): 
$e_{\lambda}^{a}=$

$I_{O}\left(\frac{\varepsilon_{\mathrm{H}_{2} \mathrm{O}_{2}}\left[\mathrm{H}_{2} \mathrm{O}_{2}\right]+\varepsilon_{\mathrm{HO}_{2}^{-}}\left[\mathrm{HO}_{2}^{-}\right]}{\varepsilon_{\mathrm{H}_{2} \mathrm{O}_{2}}\left[\mathrm{H}_{2} \mathrm{O}_{2}\right]+\varepsilon_{\mathrm{HO}_{2}^{-}}\left[\mathrm{HO}_{2}^{-}\right]+\varepsilon_{T O C_{1}}\left[\mathrm{TOC}_{1}\right]}\right)\left(1-e^{\left(-2.303 b\left(\varepsilon_{\mathrm{H}_{2} \mathrm{O}_{2}}\left[\mathrm{H}_{2} \mathrm{O}_{2}\right]+\varepsilon_{\mathrm{HO}_{2}^{-}}\left[\mathrm{HO}_{2}^{-}\right]+\varepsilon_{\mathrm{TOC}_{1}}\left[\mathrm{TOC}_{1}\right]\right)\right)}\right)$

where $I_{o}$, the intensity of the incident light, was calculated to be $1.97 \times 10^{-5}$ Einstein $\mathrm{L}^{-1} \mathrm{~s}^{-1}$, which is the light incident at $254 \mathrm{~nm}$ photon irradiance estimated from $14 \mathrm{~W}$ LP lamp over the photoreactor volume assuming $10 \%$ attenuation by the quartz sleeve and $33 \%$ efficiency for the LP lamp (Sharpless and Linden, 2003). The fraction shows the portion of the UV radiation absorption by hydrogen peroxide. Parameters $\varepsilon_{H_{2} O_{2}}, \varepsilon_{H O_{2}^{-}}$, and $\varepsilon_{T O C_{1}}$ are the molar extinction coefficients of $\mathrm{H}_{2} \mathrm{O}_{2}\left(18.7 \mathrm{M}^{-1} \mathrm{~cm}^{-1}\right)^{21}, \mathrm{HO}_{2}^{-}\left(210 \mathrm{M}^{-1} \mathrm{~cm}^{-1}\right)^{21}, \mathrm{TOC}_{1}\left(213.96 \mathrm{M}^{-1} \mathrm{~cm}^{-1}\right)$ at 254 $\mathrm{nm}$, and $b$ is the effective path length (annular space) in the photoreactor. The parameter $\varepsilon_{T O C_{1}}$ was determined by measuring the absorbance of several dilutions of the PEO in distilled water by means of a spectrophotometer (Ultrospec 1100 pro, 66Amersham Biosciences, USA). The absorbance at $254 \mathrm{~nm}\left(A_{254}\right)$ is then related to the extinction coefficient $(\varepsilon)$ as follows:

$A_{254}=\varepsilon_{T O C_{1}} c_{T O C_{1}} l$

where $C_{T O C_{1}}$ and $l$ are the TOC concentration of PEO and the effective path length of the UV radiation in the spectrophotometer $(1 \mathrm{~cm})$, respectively. Therefore, the kinetic expressions are obtained by incorporating the decomposition and the production of the species through freeradical reactions as shown below:

$$
\begin{aligned}
& R_{\mathrm{H}_{2} \mathrm{O}_{2}}= \\
& \left(-\mathrm{R}_{U V, \mathrm{H}_{2} \mathrm{O}_{2}}-k_{2}\left[^{\bullet} \mathrm{OH}\right]\left[\mathrm{H}_{2} \mathrm{O}_{2}\right]-k_{3}\left[\bullet^{\bullet} \mathrm{OH}\right]\left[\mathrm{HO}_{2}^{-}\right]-k_{4}\left[\mathrm{HO}_{2}^{\bullet}\right]\left[\mathrm{H}_{2} \mathrm{O}_{2}\right]-k_{5}\left[\mathrm{O}_{2}^{\bullet-}\right]\left[\mathrm{H}_{2} \mathrm{O}_{2}\right]+\right. \\
& \left.k_{8}\left[{ }^{\circ} \mathrm{OH}\right]^{2}+k_{9}\left[\mathrm{HO}_{2}^{\bullet}\right]^{2}+k_{11}\left[\mathrm{HO}_{2}^{\bullet}\right]\left[\mathrm{O}_{2}^{\bullet-}\right]\right) \times\left(\frac{1}{1+K_{a}\left[\mathrm{H}^{+}\right]^{-1}}\right)
\end{aligned}
$$




$$
\begin{aligned}
& R_{\mathrm{HO}_{2}^{-}}= \\
& \left(-R_{U V, \mathrm{H}_{2} \mathrm{O}_{2}}-k_{2}\left[^{\bullet} \mathrm{OH}\right]\left[\mathrm{H}_{2} \mathrm{O}_{2}\right]-k_{3}\left[^{\bullet} \mathrm{OH}\right]\left[\mathrm{HO}_{2}^{-}\right]-k_{4}\left[\mathrm{HO}_{2}^{\bullet}\right]\left[\mathrm{H}_{2} \mathrm{O}_{2}\right]-k_{5}\left[\mathrm{O}_{2}^{\bullet-}\right]\left[\mathrm{H}_{2} \mathrm{O}_{2}\right]+\right. \\
& \left.k_{8}\left[{ }^{\bullet} \mathrm{OH}\right]^{2}+k_{9}\left[H O_{2}^{\bullet}\right]^{2}+k_{11}\left[H O_{2}^{*}\right]\left[\mathrm{O}_{2}^{\bullet-}\right]\right) \times\left(\frac{1}{1+K_{a}^{-1}\left[H^{+}\right]}\right) \\
& R \cdot \bullet_{\mathrm{OH}}=2 R_{U V, \mathrm{H}_{2} \mathrm{O}_{2}}-k_{2}\left[{ }^{\circ} \mathrm{OH}\right]\left[\mathrm{H}_{2} \mathrm{O}_{2}\right]-k_{3}\left[{ }^{\circ} \mathrm{OH}\right]\left[\mathrm{HO}_{2}^{-}\right]+k_{4}\left[\mathrm{HO}_{2}^{\bullet}\right]\left[\mathrm{H}_{2} \mathrm{O}_{2}\right]+
\end{aligned}
$$

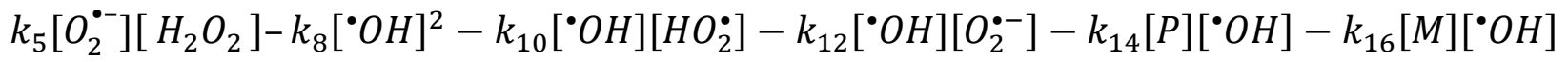

$$
\begin{aligned}
& R_{\mathrm{HO}_{2}^{\circ}}=k_{2}\left[^{\bullet} \mathrm{OH}\right]\left[\mathrm{H}_{2} \mathrm{O}_{2}\right]+k_{3}\left[^{\bullet} \mathrm{OH}\right]\left[\mathrm{HO}_{2}^{-}\right]-k_{4}\left[\mathrm{HO}_{2}^{\bullet}\right]\left[\mathrm{H}_{2} \mathrm{O}_{2}\right]+k_{6}\left[\mathrm{O}_{2}^{\bullet-}\right]\left[\mathrm{H}^{+}\right]-k_{7}\left[\mathrm{HO}_{2}^{*}\right]- \\
& k_{9}\left[\mathrm{HO}_{2}^{*}\right]^{2}-k_{10}\left[^{\bullet} \mathrm{OH}\right]\left[\mathrm{HO}_{2}^{*}\right]-k_{11}\left[\mathrm{HO}_{2}^{*}\right]\left[\mathrm{O}_{2}^{\bullet-}\right] \\
& R_{\mathrm{O}_{2}^{--}}=-k_{5}\left[\mathrm{O}_{2}^{\bullet-}\right]\left[\mathrm{H}_{2} \mathrm{O}_{2}\right]-k_{6}\left[\mathrm{O}_{2}^{\bullet-}\right]\left[\mathrm{H}^{+}\right]+k_{7}\left[\mathrm{HO}_{2}^{\bullet}\right]-k_{11}\left[\mathrm{HO}_{2}^{\bullet}\right]\left[\mathrm{O}_{2}^{\bullet-}\right]-k_{12}\left[{ }^{\bullet} \mathrm{OH}\right]\left[\mathrm{O}_{2}^{\bullet-}\right]
\end{aligned}
$$

Also, the reaction rates of the polymer, the polymer radical, and the monomer could be written by the following equations:

$$
\begin{aligned}
& R_{p}(n, t)=-k_{14}(n)\left[\bullet^{\bullet} \mathrm{OH}\right] p(n, t) \\
& R_{r}(n, t)=k_{14}(n)\left[^{\bullet} \mathrm{OH}\right] p(n, t)-k_{15}(n) r(n, t)+\int_{n}^{\infty} k_{15}\left(n^{\prime}\right) r\left(n^{\prime}, t\right) \delta\left(n-\left(n^{\prime}-1\right)\right) \mathrm{d} n^{\prime} \\
& R_{m}(n, t)=\int_{n}^{\infty} k_{15}\left(n^{\prime}\right) r\left(n^{\prime}, t\right) \Omega\left(1, n^{\prime}\right) \mathrm{d} n^{\prime}-k_{16}(n)[\cdot O H] m(n, t)
\end{aligned}
$$

In these equations, $p(n, t), r(n, t)$, and $m(n, t)$ represent the chain length distribution of the polymer, the chain end radical, and the monomer, respectively. At small reaction times, all rate coefficients are usually assumed to be independent of the chain length and they could be 
considered constant (Madras et al., 1997; McCoy and Madras, 1997). In order to make the above equations easy to solve, a moment operation is used as follows:

$r^{(p)}(t)=\int_{0}^{\infty} r(n, t) n^{p} \mathrm{~d} n$

The zero moment $(p=0)$ is the time-dependent molar concentration of the polymer and the first moment $(p=1)$ is the mass concentration. Therefore, applying moment operation results in the following equations:

$R_{p^{(p)}}=-k_{14}\left[{ }^{\bullet} \mathrm{OH}\right] p^{(p)}$

$R_{r(p)}=k_{14}\left[{ }^{\bullet} O H\right] p^{(p)}-k_{15} r^{(p)}+k_{15} \sum_{j=0}^{k}(-1)^{j} r^{(k-j)}$

$R_{m}^{(p)}=k_{15} r^{(0)}-k_{16}\left[{ }^{\bullet} O H\right] m^{(p)}$

As mentioned previously, zero moment represents the molar concentration of the polymer, where the TOC concentration is considered as the output parameter (or response). Therefore, zero moment was applied to Equations (4.47) to (4.49) and $\mathrm{TOC}_{1}$ and $\mathrm{TOC}_{2}$ concentrations were considered as the molar concentrations of the polymer and the monomer as follows:

$$
\begin{aligned}
& R_{\text {TOC }_{1}}=-k_{14}\left[{ }^{\bullet} \mathrm{OH}\right]\left[\mathrm{TOC}_{1}\right] \\
& R_{r^{(0)}}=k_{14}\left[{ }^{\bullet} \mathrm{OH}\right]\left[\mathrm{TOC}_{1}\right] \\
& R_{\text {TOC }_{2}}=k_{15} r^{(0)}-k_{16}\left[{ }^{\bullet} \mathrm{OH}\right]\left[\mathrm{TOC}_{2}\right]
\end{aligned}
$$

Therefore, Equation (4.13) must be modified as follows: 
$R \cdot \bullet_{\mathrm{OH}}=2 R_{U V, \mathrm{H}_{2} \mathrm{O}_{2}}-k_{2}\left[^{\bullet} \mathrm{OH}\right]\left[\mathrm{H}_{2} \mathrm{O}_{2}\right]-k_{3}\left[^{\bullet} \mathrm{OH}\right]\left[\mathrm{HO}_{2}^{-}\right]+k_{4}\left[\mathrm{HO}_{2}^{\bullet}\right]\left[\mathrm{H}_{2} \mathrm{O}_{2}\right]+k_{5}\left[\mathrm{O}_{2}^{--}\right]\left[\mathrm{H}_{2} \mathrm{O}_{2}\right]-$

$k_{8}\left[^{\cdot} \mathrm{OH}\right]^{2}-k_{10}\left[^{\bullet} \mathrm{OH}\right]\left[\mathrm{HO}_{2}^{\cdot}\right]-k_{12}\left[^{\bullet} \mathrm{OH}\right]\left[\mathrm{O}_{2}^{\cdot-}\right]-k_{14}\left[\mathrm{TOC}_{1}\right]\left[{ }^{\bullet} \mathrm{OH}\right]-k_{16}\left[\mathrm{TOC}_{2}\right]\left[{ }^{\circ} \mathrm{OH}\right]$

The general mass balance equation given by Equation (4.27) is used to interpret the experimental data in the batch recirculation system (Bird, 2002):

$\frac{\partial C_{i}}{\partial t}+\nabla \cdot N_{i}=R_{i}$

where $C_{i}$ is the molar concentration of component $i$ in the reacting system (M), $t$ is the time, $N_{i}$ is the molar flux of component $i\left(\mathrm{~mol} \mathrm{~cm}^{-2} \mathrm{~s}^{-1}\right)$, and $R_{i}$ is the homogeneous molar reaction rate of the component $i\left(\mathrm{M}^{-1} \mathrm{~s}^{-1}\right)$, respectively. The general mass balance equation must be applied to the recirculating batch photoreactor. If the system works under the well-stirred conditions (the ratio of the photoreactor volume to the total volume $\left(\frac{V_{P}}{V_{T}}\right) \ll 1$, that is 0.092 in this case) and high recirculating flow rate to ensure small conversion per pass (less than 5\%), the rate of the change of the concentration in the tank could be written as follows (Labas et al., 2002):

$\frac{d C_{i}}{d t}=\frac{V_{R}}{V_{T}}\left(R_{i}\right)$

Therefore, Equations (4.38), (4.39), (4.41), and (4.42) along with the Equations (4.50)-(4.53) are substituted into the Equation (4.55) for each species and the obtained set of stiff ordinary differential equations are solved simultaneously. The model was solved using the MATLAB 7.10 (R2010) environment based on the Gear's method (Appendix B). The numerical algorithm is a variable-order solver based on the numerical differentiation formulas. The proposed model needs to be validated. 


\subsubsection{Model Validation}

In order to validate the proposed model, the model predictions are compared to the experimental data carried out at different operating conditions based on the results of the experimental design. The most influential factors were found to be the initial PEO concentration and the initial $\mathrm{H}_{2} \mathrm{O}_{2}$ dosage as shown in Table 4.4. The $\mathrm{pH}$ was not controlled as its effect was less pronounced in comparison to other factors. Therefore, a set of experiments were carried out at different initial PEO concentrations $\left(10,20,30\right.$, and $\left.50 \mathrm{mg} \mathrm{L}^{-1}\right)$ and initial $\mathrm{H}_{2} \mathrm{O}_{2}$ dosages $(300,500,700,900$, and $1,100 \mathrm{mg} \mathrm{L}^{-1}$ ). The recirculation rate was kept constant at $2 \mathrm{~L} \mathrm{~min}^{-1}$ to have small conversion per pass and the $\mathrm{pH}$ was at the natural $\mathrm{pH}$ of the solution $(5.5 \pm 0.5)$. Therefore, the experimentally determined values of the normalized TOC were compared with the model predictions in Figure 4.18 to Figure 4.22. As illustrated in Figure 4.18 to Figure 4.22, the proposed model provided accurate predictions of the percent TOC removal as a function of reaction time suggesting that no major contributor to the overall kinetic model was ignored. Also, Figure 4.18 to Figure 4.22 show that as the initial concentration of PEO increases (for all the initial $\mathrm{H}_{2} \mathrm{O}_{2}$ dosages) the percent TOC removal decreases. As mentioned earlier, higher UV radiation absorption by PEO results in less UV penetration into the solution. Therefore, less UV radiation is available for $\mathrm{H}_{2} \mathrm{O}_{2}$ to generate oxidant radicals such as ${ }^{\circ} \mathrm{OH}$. 


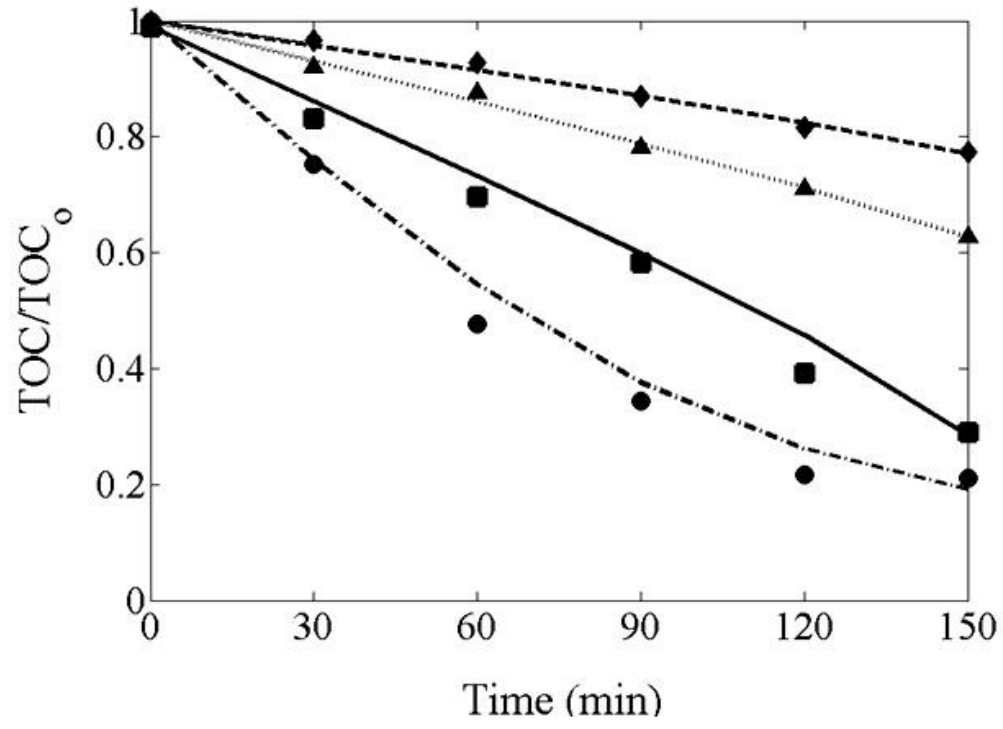

Figure 4.18. Comparison of experimental data $\left(\bullet: 10 \mathrm{mg} \mathrm{L}^{-1} \mathrm{PEO} ; \mathbf{\boldsymbol { }}: 20 \mathrm{mg} \mathrm{L}^{-1} \mathrm{PEO} ; \boldsymbol{\Delta}: 30 \mathrm{mg}\right.$ $\mathrm{L}^{-1} \mathrm{PEO}$; $50 \mathrm{mg} \mathrm{L}^{-1} \mathrm{PEO}$ ) and corresponding model predictions (lines) of $\mathrm{TOC}^{\mathrm{TOC}} \mathrm{C}_{\mathrm{o}}$ as a function of time for $\left[\mathrm{H}_{2} \mathrm{O}_{2}\right]_{\mathrm{o}}=300 \mathrm{mg} \mathrm{L}^{-1}$.

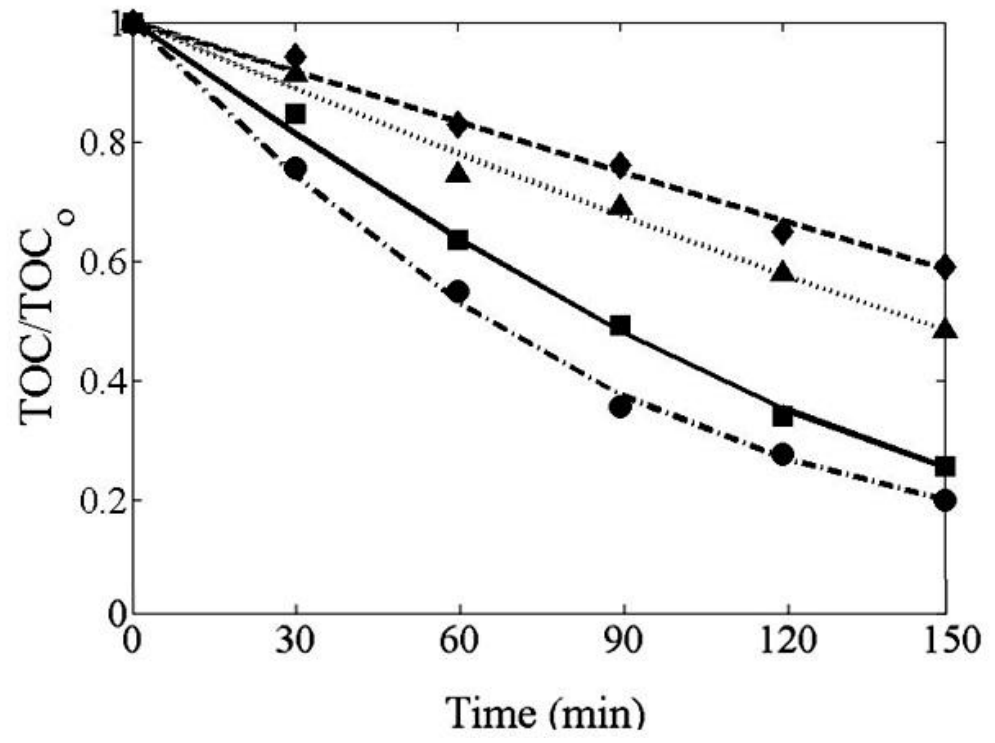

Figure 4.19. Comparison of experimental data (•:10 $\mathrm{mg} \mathrm{L}^{-1} \mathrm{PEO} ; \boldsymbol{\varpi}: 20 \mathrm{mg} \mathrm{L}^{-1} \mathrm{PEO} ; \mathbf{\Delta}: 30 \mathrm{mg}$ $\mathrm{L}^{-1} \mathrm{PEO}$; $50 \mathrm{mg} \mathrm{L}^{-1} \mathrm{PEO}$ ) and corresponding model predictions (lines) of $\mathrm{TOC}^{\mathrm{TOOC}} \mathrm{o}$ as a function of time for $\left[\mathrm{H}_{2} \mathrm{O}_{2}\right]_{\mathrm{o}}=500 \mathrm{mg} \mathrm{L}^{-1}$. 


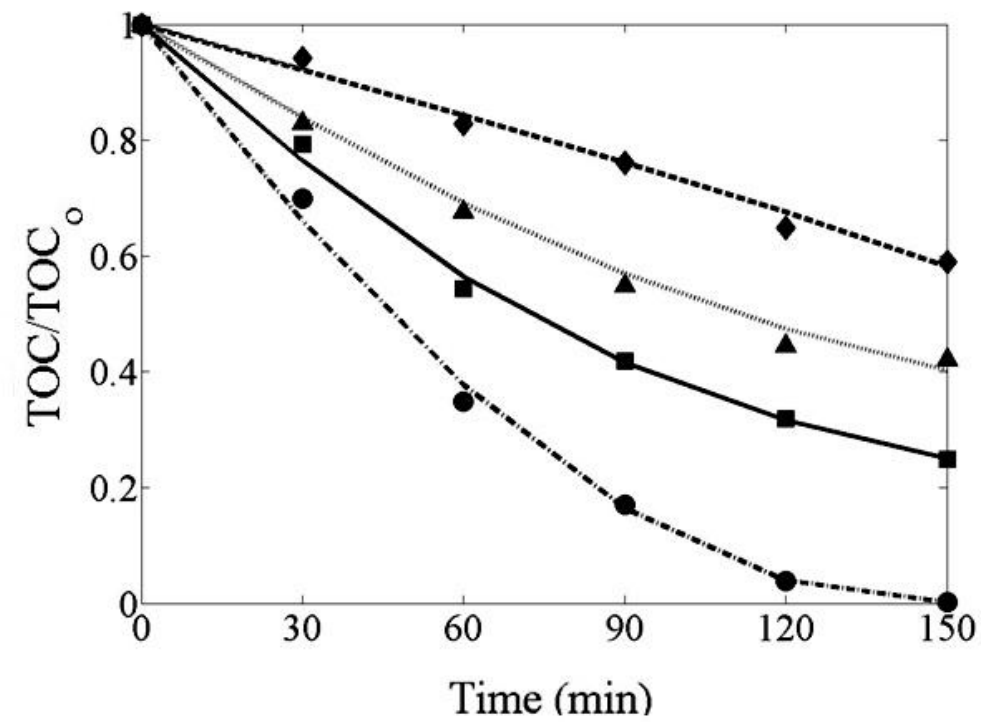

Figure 4.20. Comparison of experimental data $\left(\bullet: 10 \mathrm{mg} \mathrm{L}^{-1} \mathrm{PEO} ; \mathbf{m}: 20 \mathrm{mg} \mathrm{L}^{-1} \mathrm{PEO} ; \mathbf{\Lambda}: 30 \mathrm{mg}\right.$ $\mathrm{L}^{-1} \mathrm{PEO} ;: 50 \mathrm{mg} \mathrm{L}^{-1} \mathrm{PEO}$ ) and corresponding model predictions (lines) of $\mathrm{TOC} / \mathrm{TOC}_{\mathrm{o}}$ as a function of time for $\left[\mathrm{H}_{2} \mathrm{O}_{2}\right]_{\mathrm{o}}=700 \mathrm{mg} \mathrm{L}^{-1}$.

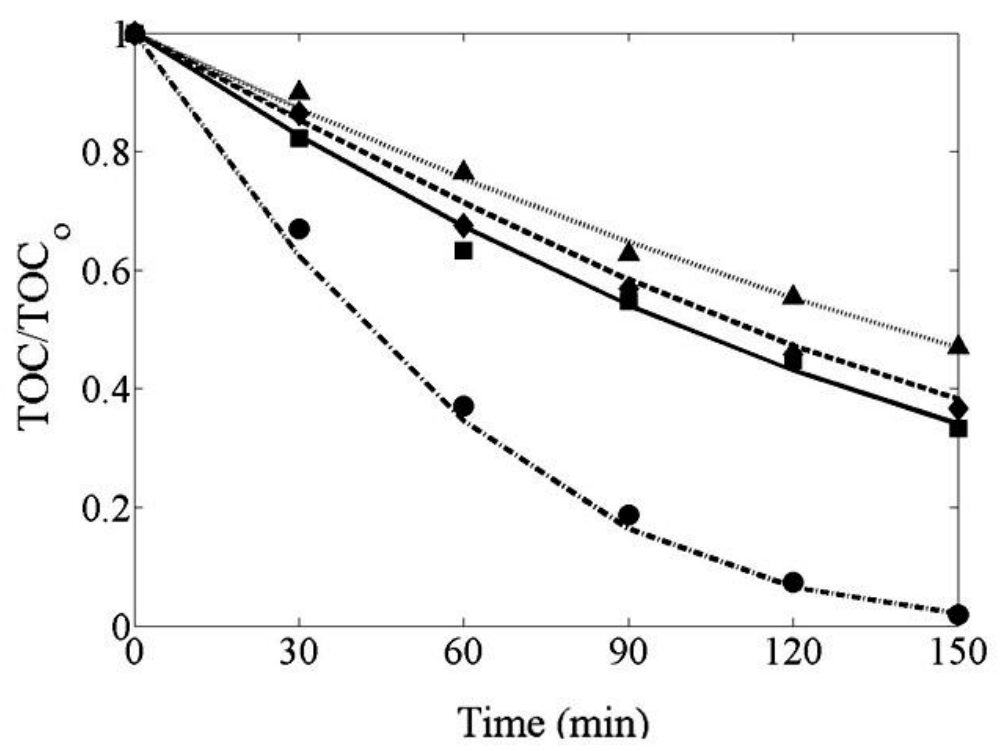

Figure 4.21. Comparison of experimental data $\left(\bullet: 10 \mathrm{mg} \mathrm{L}^{-1} \mathrm{PEO} ; \boldsymbol{\varpi}: 20 \mathrm{mg} \mathrm{L}^{-1} \mathrm{PEO} ; \mathbf{\Lambda}: 30 \mathrm{mg}\right.$ $\mathrm{L}^{-1} \mathrm{PEO} ; \bullet: 50 \mathrm{mg} \mathrm{L}^{-1} \mathrm{PEO}$ ) and corresponding model predictions (lines) of $\mathrm{TOC} / \mathrm{TOC}_{\mathrm{o}}$ as a function of time for $\left[\mathrm{H}_{2} \mathrm{O}_{2}\right]_{\mathrm{o}}=900 \mathrm{mg} \mathrm{L}^{-1}$. 


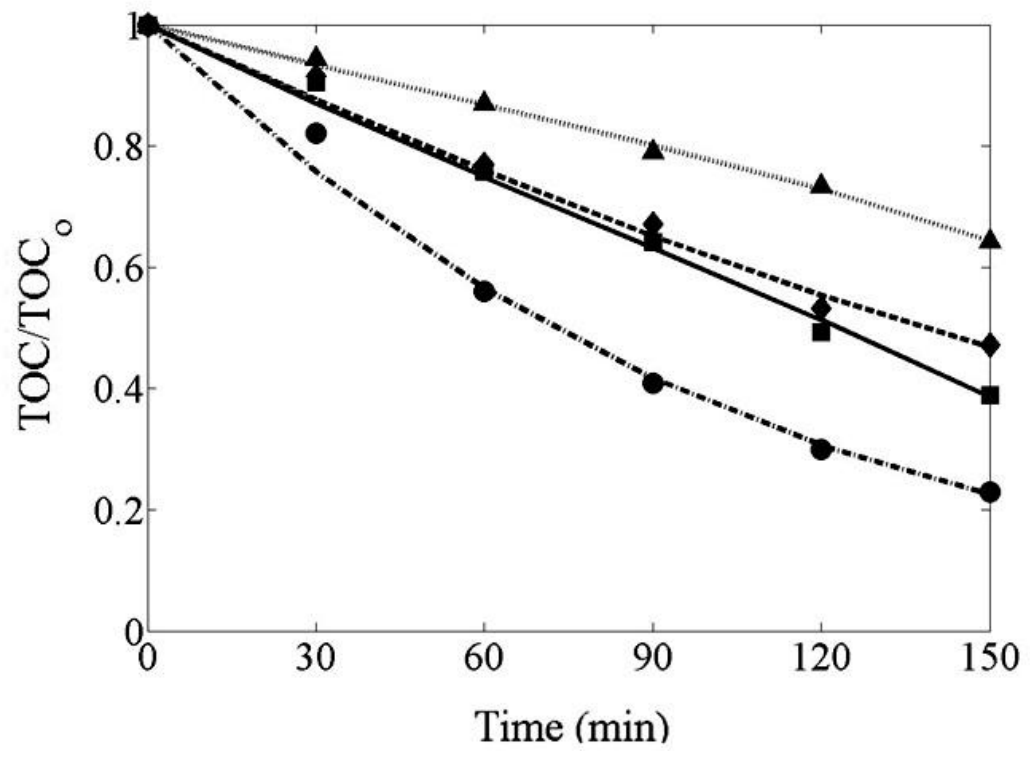

Figure 4.22. Comparison of experimental data (•:10 $\mathrm{mg} \mathrm{L}^{-1} \mathrm{PEO} ; \boldsymbol{\varpi}: 20 \mathrm{mg} \mathrm{L}^{-1} \mathrm{PEO} ; \mathbf{\Delta}: 30 \mathrm{mg}$ $\mathrm{L}^{-1}$ PEO; $50 \mathrm{mg} \mathrm{L}^{-1} \mathrm{PEO}$ ) and corresponding model predictions (lines) of $\mathrm{TOC}^{\mathrm{TOC}} \mathrm{C}_{\mathrm{o}}$ as a function of time for $\left[\mathrm{H}_{2} \mathrm{O}_{2}\right]_{\mathrm{o}}=1100 \mathrm{mg} \mathrm{L}^{-1}$. 
The initial $\mathrm{H}_{2} \mathrm{O}_{2}$ concentration is also a significant parameter in the $\mathrm{UV} / \mathrm{H}_{2} \mathrm{O}_{2}$ process. The effect of the initial $\mathrm{H}_{2} \mathrm{O}_{2}$ dosage on the percent TOC removal of PEO was also studied by varying the initial $\mathrm{H}_{2} \mathrm{O}_{2}$ dosage from 300 to $1100 \mathrm{mg} \mathrm{L}^{-1}$ for initial PEO concentrations from 10 to $50 \mathrm{mg} \mathrm{L}^{-1}$. As is evident, there is an optimum value for the initial $\mathrm{H}_{2} \mathrm{O}_{2}$ dosage above which the percent TOC removal decreases. For initial PEO concentrations of 10,20 , and $30 \mathrm{mg} \mathrm{L}^{-1}$, the optimum initial dosage of $\mathrm{H}_{2} \mathrm{O}_{2}$ was found to be $700 \mathrm{mg} \mathrm{L}^{-1}$, but for the initial PEO concentration of 50 mg L $\mathrm{L}^{-1}$, the optimum initial $\mathrm{H}_{2} \mathrm{O}_{2}$ concentration was $900 \mathrm{mg} \mathrm{L}^{-1}$ as illustrated in Figure 4.23. This finding indicates that as the initial PEO concentration increases, more $\mathrm{H}_{2} \mathrm{O}_{2}$ is needed to achieve a maximum percent TOC removal. An excess amount of $\mathrm{H}_{2} \mathrm{O}_{2}$ acts as a scavenger of hydroxyl radicals (Reaction (R2) in Table 4.1) and the formation of hydroperoxyl radicals $\left(\mathrm{HO}_{2}^{\circ}\right)$, which are much less reactive than hydroxyl radicals. Also, the excess amount of hydroperoxyl radical itself acts as a scavenger of hydroxyl radicals as shown in Reaction (R10) and subsequently reduces the degradation rate. The algorithm used for simulation and the kinetic parameter estimation is illustrated in Figure 4.24.

\subsubsection{Kinetic Parameter Estimation}

In order to find the optimal kinetic parameters, the parameter estimation was performed according to the following objective function:

$\min J(z)=\sum_{i=1}^{N}\left(\frac{T O C_{m, i}(z)}{T O C_{i}}-1\right)^{2}$

subject to the obtained dynamic model equations. 


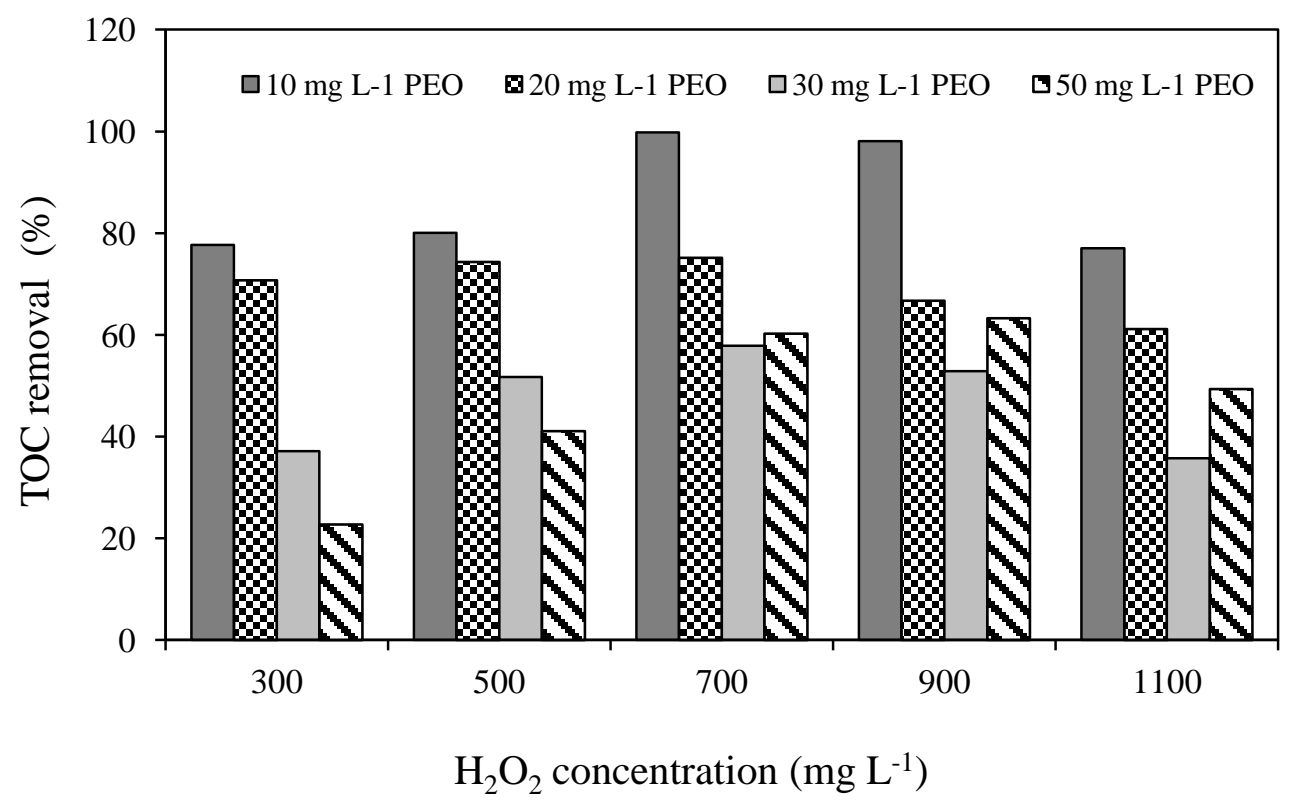

Figure 4.23. Percent TOC removal vs. initial $\mathrm{H}_{2} \mathrm{O}_{2}$ dosage for different $\mathrm{PEO}$ concentration. 


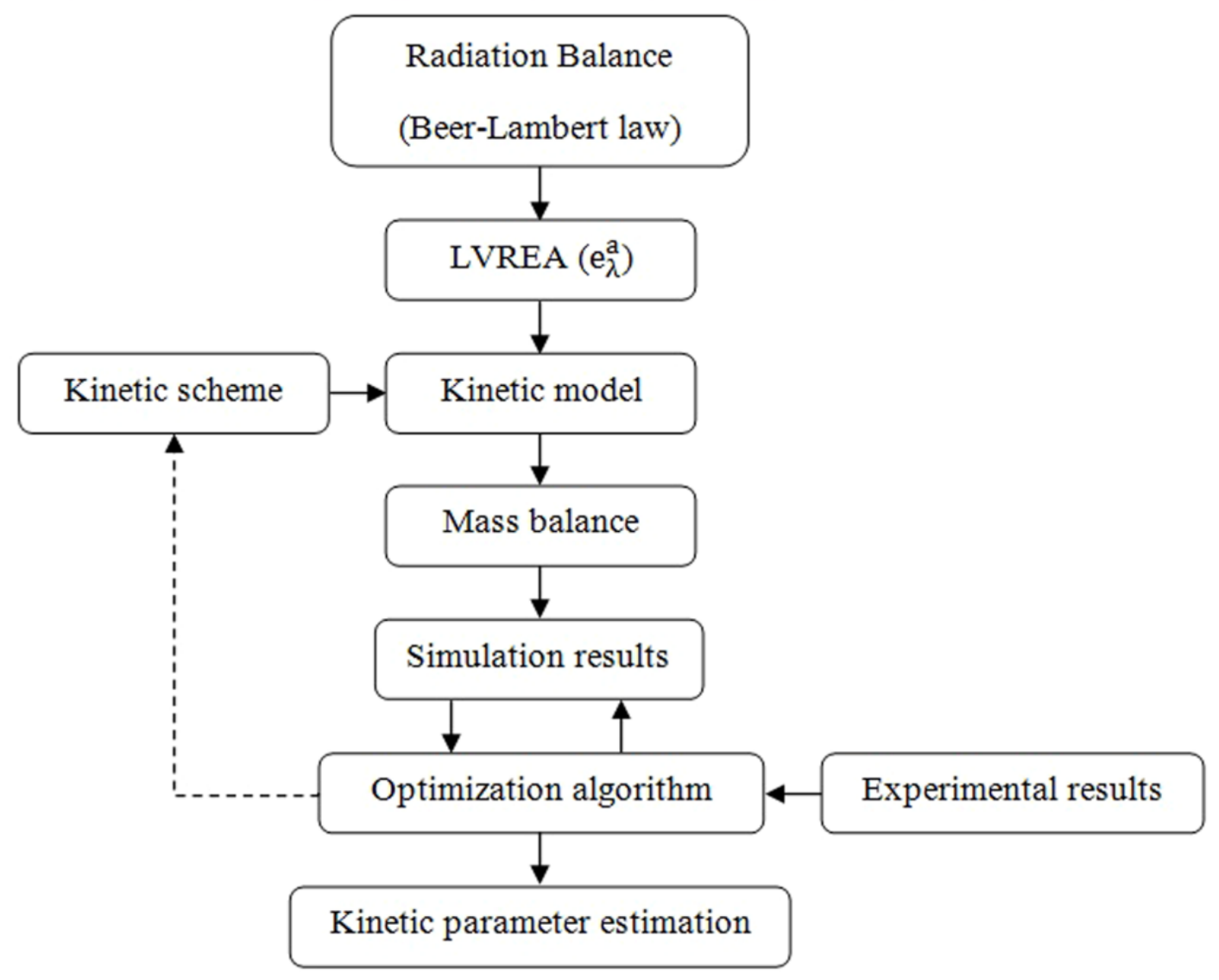

Figure 4.24. Algorithm of mathematical modeling and computational simulation procedure for kinetic parameter estimation. 
where $z=\left[k_{14}, k_{15}, k_{16}\right]^{T}$

Here, $J(z)$ is the objective function, $T O C_{m, i}(\mathrm{z})$ and $T O C_{i}$ are the model prediction and the experimental data for TOC, and $N$ is the number of data points.

The kinetic parameter estimation was carried out for 20 experimental runs with specific operating conditions. Six experimental data points at $t=0,30,60,90,120,150$ min were used for each of the 20 experimental trials. The MATLAB 7.10 (R2010) optimization toolbox was employed to solve the constrained optimization problem. Solving this problem results in obtaining optimal values of the kinetic parameter $z$ as defined in Equation (4.30).

The goodness of fit between the experimental data and the model prediction was calculated according to the Theil's inequality coefficient (TIC) as follows:

$T I C=\frac{\sqrt{\sum_{i}\left(T O C_{i}-T O C_{m, i}(z)\right)^{2}}}{\sqrt{\sum_{i} T^{2} C_{i}^{2}}+\sqrt{\sum_{i} T O C_{m, i}{ }^{2}(z)}}$

where $\mathrm{TOC}_{\mathrm{m}, \mathrm{i}}(z)$ and $\mathrm{TOC}_{i}$ represent experimentally measured and the model prediction data for TOC in each simulation run, respectively. The value of TIC less than 0.3 indicates a good agreement between the experimental data and the predicted model values (Audenaert et al., 2011). The results are presented in Table 4.6. The optimization algorithm gives the values of the parameters that minimize the sum of squares of the errors between model predictions and the experimental data. The initial guesses of the rate constants were selected according to the range of acceptable values reported in the open literature (Buxton et al., 1988). It must be noted that the obtained optimal values, that are in acceptable range, are not assumed to be the global ones 
as they are highly dependent to the initial guesses. Similar to the most of optimization problems, there is no test showing that the achieved optimal values are the global ones. However, the estimated optimal values are independent of the photoreactor configuration and can be used as the starting point for designing commercial scale photoreactors.

\subsubsection{Conclusions}

The response surface methodology using four-factor with five-level CCD and quadratic programming showed reliable results for the photodegradation of PEO by the $\mathrm{UV} / \mathrm{H}_{2} \mathrm{O}_{2}$ process. Predicted results from the response functions were in good agreement with the experimental data confirming the reliability of the employed methodology. Furthermore, results indicated that the initial PEO concentration, the initial $\mathrm{H}_{2} \mathrm{O}_{2}$ dosage, and $\mathrm{pH}$ had considerable influence on the TOC removal efficiency. The recirculation rate had no effect on the response function. From analysis of contour plots, it was observed that a decrease in the initial concentration of PEO, an increase in the initial $\mathrm{H}_{2} \mathrm{O}_{2}$ dosage up to a certain level, and an acidic $\mathrm{pH}$ condition result in an increase in the percent TOC removal. The optimum percent TOC removal (84\%) was found after $150 \mathrm{~min}$ at the operating conditions of $10 \mathrm{mg} \mathrm{L}^{-1} \mathrm{PEO}, 780 \mathrm{mg} \mathrm{L}^{-1} \mathrm{H}_{2} \mathrm{O}_{2}, \mathrm{pH} 3$, and $0.5 \mathrm{~L} \mathrm{~min}^{-1}$ recirculation rate based on the developed quadratic model. A mathematical model was developed based on the mass balance of the main species present in water to predict the percent TOC removal of PEO. The continuous distribution was used to establish the kinetic modeling of PEO based on the TOC as a surrogate parameter. The PEO initial concentration and the initial $\mathrm{H}_{2} \mathrm{O}_{2}$ dosage as the most influential factors in the percent TOC removal were varied in the second experimental part to validate the model. An excellent agreement between the model predictions 
and the experimental data was confirmed by calculating the TIC for each experimentally tested parameter. The kinetic parameters were estimated using an optimization algorithm. The obtained kinetic parameters were found to be in the acceptable range of reported values in the open literature. However, they are not assumed to be the global ones. Therefore, the proposed model explaining the free-radical induced degradation of PEO by the $\mathrm{UV} / \mathrm{H}_{2} \mathrm{O}_{2}$ process could be characterized as an accurate and interpretable model that could be used as a base for the future studies with the goal of process optimization. 
Table 4.6. Optimization and simulation results for the kinetic rate constants.

\begin{tabular}{|c|c|c|c|c|c|}
\hline \multirow{2}{*}{$\begin{array}{l}\mathrm{H}_{2} \mathrm{O}_{2} \text { Dosage } \\
\left(\mathrm{mg} \mathrm{L}^{-1}\right)\end{array}$} & \multirow{2}{*}{$\begin{array}{l}\text { PEO } \\
\text { Concentration } \\
\left(\mathbf{m g ~ L}^{-1}\right)\end{array}$} & \multicolumn{3}{|c|}{ Rate Constants } & \multirow[t]{2}{*}{ TIC } \\
\hline & & $k_{14} \times 10^{-8}\left(\mathrm{M}^{-1} \mathrm{~s}^{-1}\right)$ & $k_{15} \times 10^{-2}\left(\mathrm{~s}^{-1}\right)$ & $k_{16} \times 10^{-6}\left(\mathrm{M}^{-1} \mathrm{~s}^{-1}\right)$ & \\
\hline \multirow[t]{4}{*}{300} & 10 & 24.61 & 10.79 & 2.04 & 0.0434 \\
\hline & 20 & 15.34 & 6.5 & 3.51 & 0.0177 \\
\hline & 30 & 5.58 & 8.3 & 1.79 & 0.0053 \\
\hline & 50 & 4.16 & 7.6 & 1.53 & 0.0254 \\
\hline \multirow[t]{4}{*}{500} & 10 & 12.16 & 6.25 & 4.25 & 0.0135 \\
\hline & 20 & 18.79 & 13.18 & 1.97 & 0.0123 \\
\hline & 30 & 8.06 & 0.13 & 1.76 & 0.0099 \\
\hline & 50 & 6.35 & 2.36 & 1.56 & 0.0369 \\
\hline \multirow[t]{4}{*}{700} & 10 & 23.93 & 52.5 & 46.58 & 0.0326 \\
\hline & 20 & 24.58 & 14.01 & 32.29 & 0.0157 \\
\hline & 30 & 16.94 & 11.89 & 5.68 & 0.0338 \\
\hline & 50 & 20.50 & 15.69 & 1.76 & 0.0105 \\
\hline \multirow[t]{4}{*}{900} & 10 & 22.32 & 35.1 & 38.41 & 0.0668 \\
\hline & 20 & 25.57 & 2.88 & 4.57 & 0.0172 \\
\hline & 30 & 4.16 & 1.25 & 8.99 & 0.0118 \\
\hline & 50 & 6.01 & 19.9 & 3.76 & 0.0145 \\
\hline \multirow[t]{4}{*}{1100} & 10 & 6.10 & 10.72 & 10.43 & 0.0288 \\
\hline & 20 & 3.15 & 28.6 & 1.44 & 0.0148 \\
\hline & 30 & 1.47 & 4.12 & 5.46 & 0.0048 \\
\hline & 50 & 6.96 & 1.22 & 8.48 & 0.0163 \\
\hline
\end{tabular}




\subsection{PHOTOASSISTED FENTON-LIKE DEGRADATION OF AQUEOUS \\ POLYACRYLIC ACID: EXPERIMENTAL DESIGN, MECHANISTIC KINETIC MODEL, AND CFD MODELING}

\subsubsection{Introduction}

Among AOTs, photo-Fenton process has been found highly effective in the degradation of aromatics, pesticides, and other bio-recalcitrant compounds in industrial wastewaters (HerneyRamirez et al., 2010). In the photo-Fenton process, $\mathrm{H}_{2} \mathrm{O}_{2}$ in a catalytic cycle with $\mathrm{Fe}$ ions as the catalyst is decomposed to highly reactive radicals $(\mathrm{OH})$ that have great oxidation potential (Pérez-Moya et al., 2008; Kusic et al., 2009; Pontes et al., 2010). The free radical species facilitate the rapid degradation of target organic compounds. However, photo-Fenton processes have been rarely studied for the degradation of high molecular weight compounds such as synthetic water-soluble polymers. Also, there is a lack of sufficient information in the open literature on the kinetic modeling of photo-Fenton process as one of the AOTs for the degradation of water-soluble polymers in wastewater because of the complexity of the degradation pathway of high molecular weight compounds. Also, the effect of inorganic anions on the overall reaction rate is generally neglected and there are only few studies considering the effects of inorganic ions on the effectiveness of photo-Fenton process (De Laat and Le, 2006; Pérez-Moya et al., 2008). Furthermore, there are few studies centered on determining the degradation kinetics of water-soluble polymers using surrogate parameters such as TOC that presents aggregated information instead of specific concentration data (Beltrán et al., 2011). Although there are some studies on the computational fluid dynamics (CFD) of AOTs which involves simultaneous modeling of chemical species mass transfer, rigorous kinetic pathway, and 
photon flux distribution (Alpert et al., 2010; Mohajerani et al., 2010; Santro et al., 2010; Trujillo et al., 2010; Duran et al., 2011; Vincent et al., 2011), there is still little information on the CFD modeling of photo-Fenton-like process. In photo-Fenton-like process unlike the photo Fenton process $\left(\mathrm{UV} / \mathrm{Fe}^{2+} / \mathrm{H}_{2} \mathrm{O}_{2}\right), \mathrm{Fe}^{3+}$ is used along with the $\mathrm{UV}$ radiation and $\mathrm{H}_{2} \mathrm{O}_{2}$. Using CFD approach, the spatial distribution of various parameters such as organic compounds, $\mathrm{H}_{2} \mathrm{O}_{2}$, and photon flux in the continuous flow photoreactor could be determined. The CFD could be combined with kinetic model equations to give more accurate prediction in both space and time. In this case, the CFD could be used as a powerful tool for the design and optimization of the fullscale reactors. Therefore, in order to achieve useful results in the CFD model, a validated kinetic model is needed.

In the first part of this section, the experimental design for the PAA photodegradation by photoFenton-like process is investigated in a batch recirculation system (Figure 3.1). In the second part, a detailed mechanistic kinetic model was developed based on extensively accepted photochemical reactions and the rate constants in photo-Fenton-like process to predict the TOC removal rate as the output variable (response). Population balance equations were applied to describe the dynamics of polymer degradation. The kinetic model was validated by comparing the model predictions with the experimental data carried out at different operating conditions. Sensitivity analysis was conducted to determine the most influential chemical reactions in the kinetic model. In the second part, CFD modeling of the continuous flow at optimum operating conditions was studied. 


\subsubsection{Experimental Procedure}

The description and operating conditions of the laboratory-scale photoreactor (Figure 3.1) are given in Table 3.1. The following protocol was pursued in conducting each experiment. The PAA solution was diluted to achieve the desired PAA concentration in a 4-L solution. The lamp was turned on for 30 min before the beginning of each experiment to stabilize the light intensity and to remove any organic background. The solution was fed to the system and the temperature was kept constant at $22^{\circ} \mathrm{C}$ during each experiment by means of a heat exchanger. The $\mathrm{pH}$ was adjusted to 3 in all experimental runs as it was proved that a higher level of dissolved ferric iron species is achieved under more acidic condition (Jung et al., 2009). The samples were taken from the collection tank at time 0 and at a time interval of 15 min during a total reaction time of 90 min. The TOC concentration of the samples was monitored by the TOC analyzer. The UV/Visible spectrometer was used to determine the extinction coefficient of PAA.

\subsubsection{Experimental Design using Box-Behnken Design}

The operating conditions to maximize the TOC removal in the photo-Fenton-like process is obtained using three-factor three-level Box-Behnken experimental design combined with response surface methodology. As mentioned in Section 3.5.1.2, the Box-Behnken design, a modified central composite design, is known as an independent, rotatable quadratic design having no fractional factorial points. In this type of design, the variable combinations are at the center and the midpoints of the edges of the variable space. Also, compared to other types of experimental design such as full factorial design, the Box-Behnken experimental design needs fewer runs. In this study, the effects of three independent variables on the TOC removal were 
investigated. The independent variables were initial concentrations of PAA $\left(X_{1}\right)$, the initial $\mathrm{Fe}^{3+}$ dosage $\left(X_{2}\right)$, and the initial concentration of $\mathrm{H}_{2} \mathrm{O}_{2}\left(X_{3}\right)$ that were coded as $-1,0$, and +1 as presented in Table 4.7. The total number of experiments was 15 which can be calculated as follows:

$N=k^{2}+k+c_{p}$

where $k$ and $c_{p}$ are the factor number and rotatable number of center point, respectively. The critical experimental levels were chosen based on the preliminary experimental results and the values found in the open literature (Rojas et al., 2010; Silva et al., 2010; Sun and Lemely, 2011).

Table 4.7. Independent variables and their coded levels based on Box-Behnken design for photoFenton-like process.

\begin{tabular}{lcccc}
\hline Independent Variables & Symbols & \multicolumn{3}{c}{ Coded Levels } \\
\cline { 3 - 4 } & & -1 & 0 & 1 \\
\hline & & & & \\
{$[\mathrm{PEO}]_{0}\left(\mathrm{mg} \mathrm{L}^{-1}\right)$} & $X_{1}$ & 30 & 40 & 60 \\
{$\left[\mathrm{Fe}^{3+}\right]_{0}\left(\mathrm{mg} \mathrm{L}^{-1}\right)$} & $X_{2}$ & 500 & 5 & 7 \\
{$\left[\mathrm{H}_{2} \mathrm{O}_{2}\right]_{0}\left(\mathrm{mg} \mathrm{L}^{-1}\right)$} & & & & 1300 \\
& & & \\
\hline
\end{tabular}

Therefore, the data from Box-Behnken design was subjected to the quadratic model, a quadratic equation that correlates the dependent and independent variables. STATISTICA (trial version 10.0) and Design-Expert (trial version 8.0) were used for regression and graphical analysis of the data. The significance of independent variables and their interactions were tested using analysis of variance (ANOVA). An alpha $(\alpha)$ level of 0.05 was used to determine the statistical 
significance in all analyses. Three-dimensional surface plots were developed while holding a variable constant in the quadratic model. The experimental and predicted values were compared to validate the model. The optimal operating conditions to maximize the TOC removal rate were also determined using a numerical technique built in the software. Table 4.8 shows the results of the three-factor three-level Box-Behnken experimental design for the percent TOC removal by the photo-Fenton-like process for aqueous poly(acrylic acid). The observed and predicted results for the percent TOC removal are also shown in Table 4.8. By applying multiple regression analysis on the design matrix and the responses given in Table 4.8 , the following quadratic equation in terms of coded factors was determined to predict the percent TOC removal:

$Y=76.25-6.88 X_{1}+4.62 X_{2}+5.10 X_{3}+0.47 X_{1} X_{2}-1.13 X_{1} X_{3}-1.88 X_{2} X_{3}-4.41 X_{1}^{2}+$

$3.56 X_{2}{ }^{2}+3.07 X_{3}^{2}$

\subsubsection{Statistical Analysis}

The significance of the fit of the quadratic equation for the experimental data was tested by means of the ANOVA as shown in Table 4.9. An alpha $(\alpha)$ level of 0.05 or $95 \%$ confidence level was used to determine the statistical significance in all analyses. Results were assessed with various descriptive statistics such as the $p$-value, the $F$-value, degree of freedom (df), the determination coefficient $\left(R^{2}\right)$, the adjusted determination coefficient $\left(R_{\text {adj }}^{2}\right)$, the sum of squares (SS), and the mean sum of squares (MSS) as presented in Table 4.9. The significance of each coefficient in Equation (4.60) was determined by the Fisher's $F$-test and values of probability > $F$. As shown in Table 4.9, a small probability value $(p<0.0110)$ indicates that the model (Equation (4.60)) was highly significant and can be used to predict response function accurately. The goodness of fit of the model was checked by determination coefficient $\left(R^{2}\right)$. The high values 
of regression (0.9461) and adjusted regression coefficients (0.8490) indicate that the developed quadratic model can adequately describe the system behavior within the selected range of operating parameters. The adequate precision, signal to noise ratio, greater than $4(10.21$ in this case) shows that the model could be used to navigate the design space defined by the BoxBehnken design.

Table 4.8. Three-factor three-level Box-Behnken design for RSM.

\begin{tabular}{|c|c|c|c|c|c|}
\hline \multirow[t]{2}{*}{ Run } & \multicolumn{3}{|c|}{ Independent Coded Variables } & \multicolumn{2}{|c|}{ TOC Removal (\%) } \\
\hline & $X_{1}$ & $X_{2}$ & $X_{3}$ & Observed & Predicted \\
\hline 1 & -1 & -1 & 0 & 78.46 & 78.13 \\
\hline 2 & 1 & -1 & 0 & 61.47 & 63.42 \\
\hline 3 & -1 & 1 & 0 & 88.37 & 86.42 \\
\hline 4 & 1 & 1 & 0 & 73.28 & 73.61 \\
\hline 5 & -1 & 0 & -1 & 72.23 & 75.55 \\
\hline 6 & 1 & 0 & -1 & 63.02 & 64.06 \\
\hline 7 & -1 & 0 & 1 & 89.06 & 88.02 \\
\hline 8 & 1 & 0 & 1 & 75.31 & 71.99 \\
\hline 9 & 0 & -1 & -1 & 74.25 & 71.27 \\
\hline 10 & 0 & 1 & -1 & 85.64 & 84.27 \\
\hline 11 & 0 & -1 & 1 & 83.86 & 85.23 \\
\hline 12 & 0 & 1 & 1 & 87.74 & 90.72 \\
\hline 13 & 0 & 0 & 0 & 76.16 & 76.25 \\
\hline 14 & 0 & 0 & 0 & 75.95 & 76.25 \\
\hline 15 & 0 & 0 & 0 & 76.64 & 76.25 \\
\hline
\end{tabular}

The normality of the data could be checked through the normal probability plot of the residuals. If the points on the plot lie on a straight line, the residuals are normally distributed as confirmed in Figure 4.25. Also, a high correlation between observed and predicted data shown in Figure 
4.26 indicates their low discrepancies. The significance of each model parameter was determined by means of Fischer's $F$-value and $p$-value.

Table 4.9. ANOVA for prediction of TOC removal rate by the quadratic model.

\begin{tabular}{lllllc}
\hline Source & Sum of & & Mean & & $\boldsymbol{p}$-value \\
& Squares & Df & Square & $\boldsymbol{F}$-Value & Prob $>\boldsymbol{F}$ \\
\hline Model & 942.9771 & 9 & 104.7752 & 9.749104 & 0.0110 \\
$X_{1}-X_{1}$ & 378.6752 & 1 & 378.6752 & 35.23489 & 0.0019 \\
$X_{2}-X_{2}$ & 171.0325 & 1 & 171.0325 & 15.9142 & 0.0104 \\
$X_{3}-X_{3}$ & 208.3861 & 1 & 208.3861 & 19.38987 & 0.0070 \\
$X_{1} X_{2}$ & 0.9025 & 1 & 0.9025 & 0.083976 & 0.7836 \\
$X_{1} X_{3}$ & 5.1529 & 1 & 5.1529 & 0.479466 & 0.5195 \\
$X_{2} X_{3}$ & 14.10003 & 1 & 14.10003 & 1.311976 & 0.3039 \\
$X_{1}{ }^{2}$ & 71.84908 & 1 & 71.84908 & 6.685399 & 0.0491 \\
$X_{2}^{2}$ & 46.6963 & 1 & 46.6963 & 4.344988 & 0.0915 \\
$X_{3}^{2}$ & 34.71467 & 1 & 34.71467 & 3.230123 & 0.1322 \\
Residual & 53.73583 & 5 & 10.74717 & & \\
Pure Error & 0.2502 & 2 & 0.1251 & & \\
Cor Total & 996.7129 & 14 & & & \\
\hline
\end{tabular}

The $F$-value is the test for comparing the curvature variance with residual variance and probability $>F$ ( $p$-value) is the probability of seeing the observed $F$-value if the null hypothesis is true. Small probability values call for rejection of the null hypothesis and the curvature is not significant. Therefore, the larger the value of $F$ and the smaller the value of $p$, the more significant is the corresponding coefficient. As shown in the Table 4.9, the $p$-value less than 0.05 indicate the model parameters are significant. Therefore, all three independent variables have significant effect on the percent TOC removal based on their $p$-value. However, the initial $\mathrm{Fe}^{3+}$ dosage showed less significant effect compared to the initial concentration of PAA and the initial 


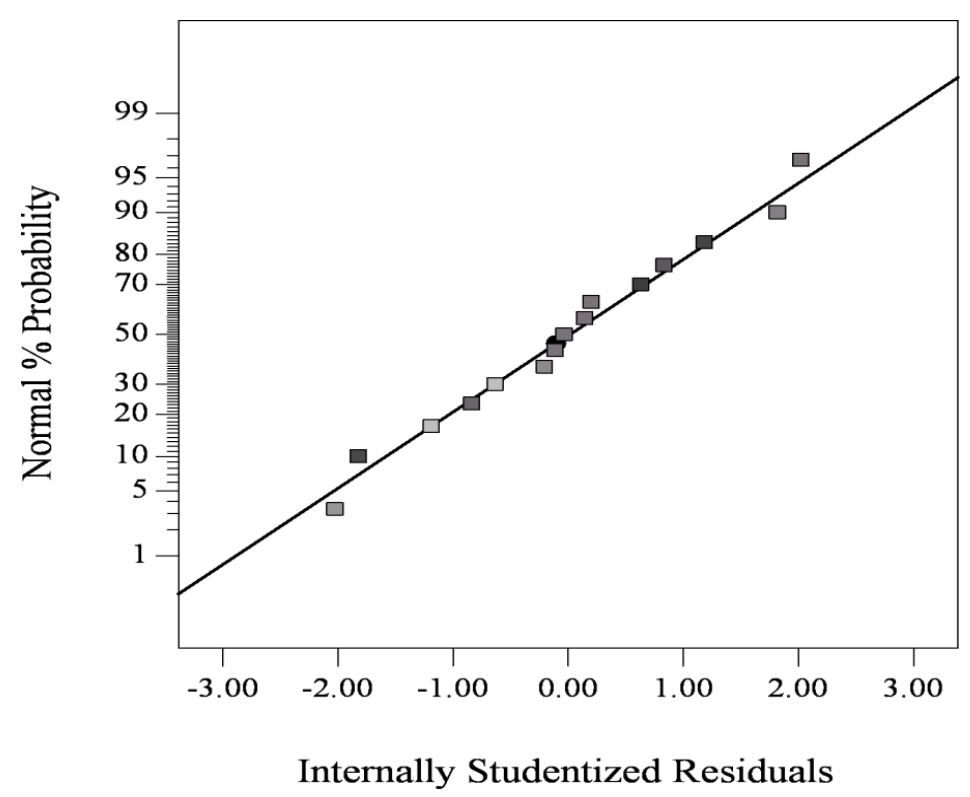

Figure 4.25. Normal probability plot of the residuals.

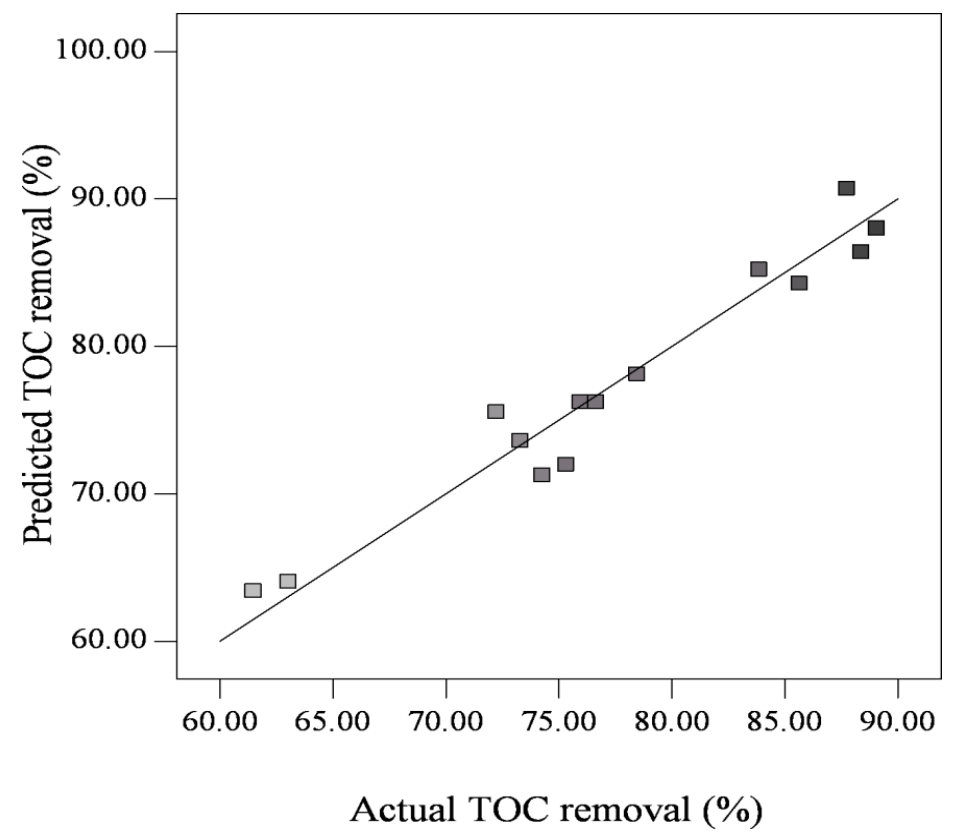

Figure 4.26. Comparison of predicted TOC removal rates versus actual values. 
$\mathrm{H}_{2} \mathrm{O}_{2}$ dosage. Also, as evident in Table 4.9, the quadratic effect of the initial PAA concentration has significant effect on the response function.

\subsubsection{Interaction Effects between the Independent Variables}

The interaction effects between the independent variables are illustrated in three dimensional (3D) response surface and two dimensional (2D) contour plots (Figure 4.27 to Figure 4.29). These figures are the graphical representations of the regression analysis. In such plots, the response function of two factors is presented while all others are at the fixed levels. As illustrated in Figure 4.27 and Figure 4.28, the higher initial concentration of PAA results in lower percent of the TOC removal which is due to the UV absorption by the polymer molecules. This would result in reducing $\mathrm{UV}$ absorption by the oxidizers $\left(\mathrm{Fe}^{3+}\right.$ and $\left.\mathrm{H}_{2} \mathrm{O}_{2}\right)$ and consequently producing less ${ }^{\circ} \mathrm{OH}$ which is the main cause of polymer degradation. However, the effect of the initial concentration of PAA is less pronounced at lower concentrations. As depicted in Figure 4.27 to Figure 4.29, the higher the concentrations of $\mathrm{Fe}^{3+}$ and $\mathrm{H}_{2} \mathrm{O}_{2}$ are the higher is the percent TOC removal which is expected based on the chain reaction in Section 2.1.1.2. Higher concentration of oxidizers leads to the higher generation of ${ }^{\circ} \mathrm{OH}$ which is responsible to attack the polymer molecules.

\subsubsection{Optimization of Process Parameters}

In order to obtain the optimum operating conditions to maximize the TOC removal (response function), Equation (4.33) is considered as the objective function. The optimization is carried out by means of the numerical technique built in the Design Expert Software (Version 8.0.5) which 
searches the design space to achieve the goal of the optimization in the range of independent factors. The optimum values to achieve the maximum $93.94 \%$ TOC removal rate after $90 \mathrm{~min}$ were $22.80 \mathrm{mg} \mathrm{L}^{-1} \mathrm{PAA}, 7 \mathrm{mg} \mathrm{L}^{-1} \mathrm{Fe}^{3+}$, and $1300 \mathrm{mg} \mathrm{L}^{-1} \mathrm{H}_{2} \mathrm{O}_{2}$. The obtained optimal operating conditions were used in another experimental run to validate the model prediction. 91.35\% TOC removal found experimentally confirms the reliability of the model.

\subsubsection{Mechanistic Kinetic Model}

Reaction mechanisms and kinetic modeling of photo-Fenton and photo-Fenton-like processes have been investigated in several studies (Devi et al., 2009; Kusic et al., 2009; Bach et al., 2010). However, the mechanisms involved may be complex and not well-established. Particularly, the effects of inorganic anions, introduced to the solutions with the reactants such as $\mathrm{FeCl}_{3}$ or $\mathrm{FeSO}_{4}$, on the overall rate of reaction are generally ignored in studying the kinetic modeling of photoFenton process (De Laat et al., 2006). In the case of chloride ion, the degradation rates of organic compounds are lower due to the inhibitory effect of the anion. Therefore, in this study, the reaction scheme for the photo-Fenton-like degradation of PAA $\left(\mathrm{UV} / \mathrm{H}_{2} \mathrm{O}_{2} / \mathrm{FeCl}_{3}\right)$ is summarized in Table 4.10 based on extensively accepted photochemical reactions and the rate constants in photo-Fenton-like process (Ghafoori et al., 2013a).

The negative effects of the chloride anion on the reaction rate can be explained by two sets of reactions in Table 4.10. The first one could be explained by Reactions (R33)-(R35) that lead to the formation of iron-chlorocomplexes $\left(\mathrm{FeCl}^{+}, \mathrm{FeCl}^{2+}, \mathrm{FeCl}_{2}^{+}\right)$. The second one is the scavenging effect of chloride anion $\left(\mathrm{Cl}^{-}\right)$on hydroxyl radicals $(\mathrm{OH})$ as shown in Reaction (R21). Also, as is evident in Reaction (R27) the scavenging effect of chloride radical $\left(\mathrm{Cl}^{\circ}\right)$ on hydrogen peroxide 
$\left(\mathrm{H}_{2} \mathrm{O}_{2}\right)$ results in the formation of hydroperoxyl radical $\left(\mathrm{HO}_{2}^{*}\right)$ which is less reactive than hydroxyl radicals. The complete mineralization of PAA monomer to $\mathrm{H}_{2} \mathrm{O}$ and $\mathrm{CO}_{2}$ disregarding other intermediates is presented in Reactions (R39) to (R41).

There is little information available in the open literature on the mechanistic kinetic modeling involving the reactions of photo-Fenton-like process by $\mathrm{UV} / \mathrm{Fe}^{3+} / \mathrm{H}_{2} \mathrm{O}_{2}$ considering chloride radicals $\left(\mathrm{Cl}^{\circ}\right)$, chloride anion $\left(\mathrm{Cl}^{-}\right)$, dichloride anion radicals $\left(\mathrm{Cl}_{2}^{--}\right)$, the formation of ironchlorocomplexes $\left(\mathrm{FeCl}^{+}, \mathrm{FeCl}^{2+}, \mathrm{FeCl}_{2}^{+}\right)$, and a detailed mathematical model for the polymer degradation. A kinetic model based on the mass balance of the species of interest in reaction steps proposed in Table 4.10 was developed. The kinetic model predicts the TOC concentration profile of the polymer as a function of time.

The model assumptions are as follows:

(i) The direct photolysis of PAA does not show any TOC removal indicating that the quantum yield in Reaction (R2) is zero, i.e., $\varphi_{P A A}=0$;

(ii) Only hydroxyl radical attack is responsible for the polymer degradation as the oxidation potential of other radicals are negligible compared to that of hydroxyl radicals (Giroto et al., 2010);

(iii) The $\mathrm{pH}$ changes during the reaction are considered negligible because of the insignificant effect on the TOC removal efficiency (Crittenden et al., 1999; Jung et al., 2009);

(iv) $\mathrm{H}_{2} \mathrm{O}_{2}$ and $\mathrm{HO}_{2}^{-}$, acid-base conjugates as shown in Reaction (20) in Table 4.10, are the main light-absorbing species; and 


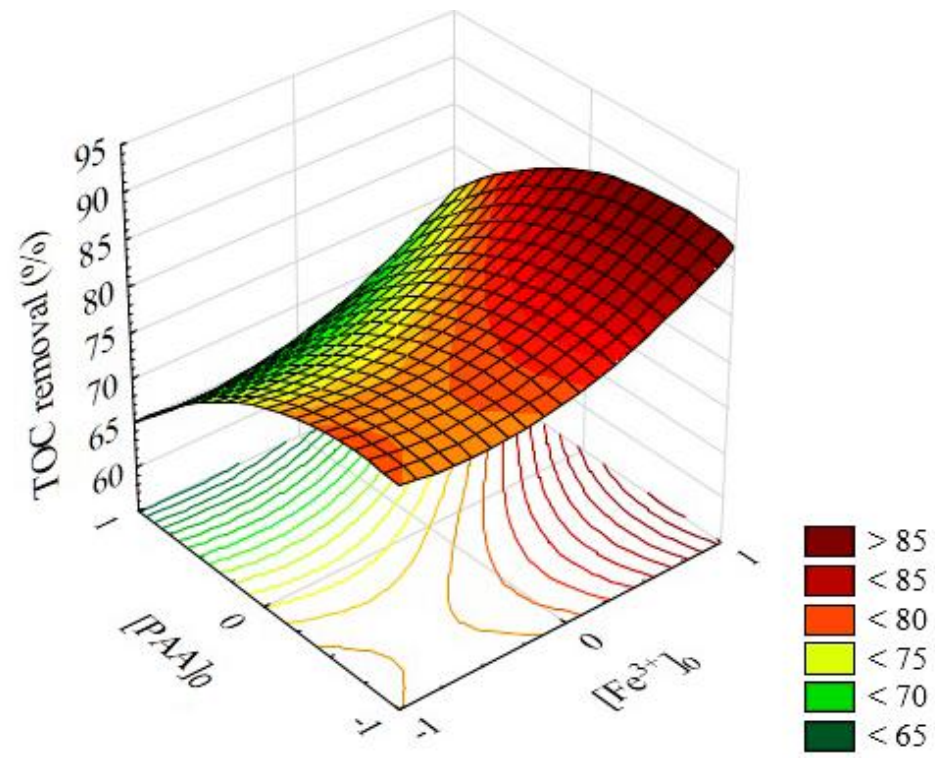

Figure 4.27. Interaction effect between $[\mathrm{PAA}]_{\mathrm{o}}$ and $\left[\mathrm{Fe}^{3+}\right]_{\mathrm{o}}$ on percent TOC removal.

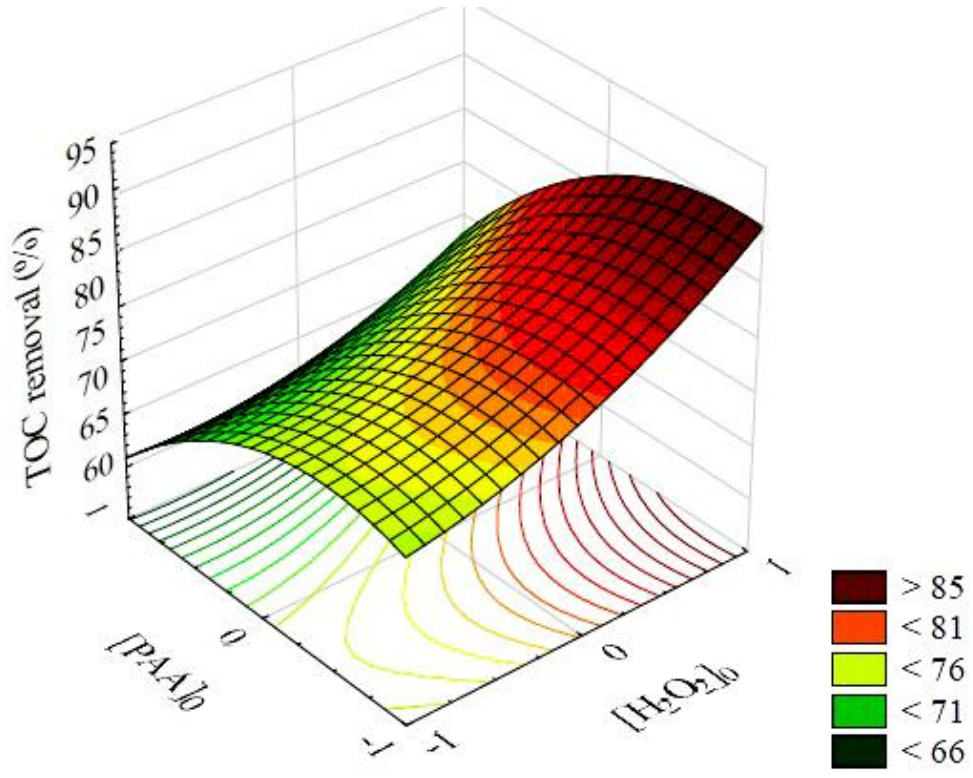

Figure 4.28. Interaction effect between $[\mathrm{PAA}]_{\mathrm{o}}$ and $\left[\mathrm{H}_{2} \mathrm{O}_{2}\right]_{\mathrm{o}}$ on percent TOC removal. 


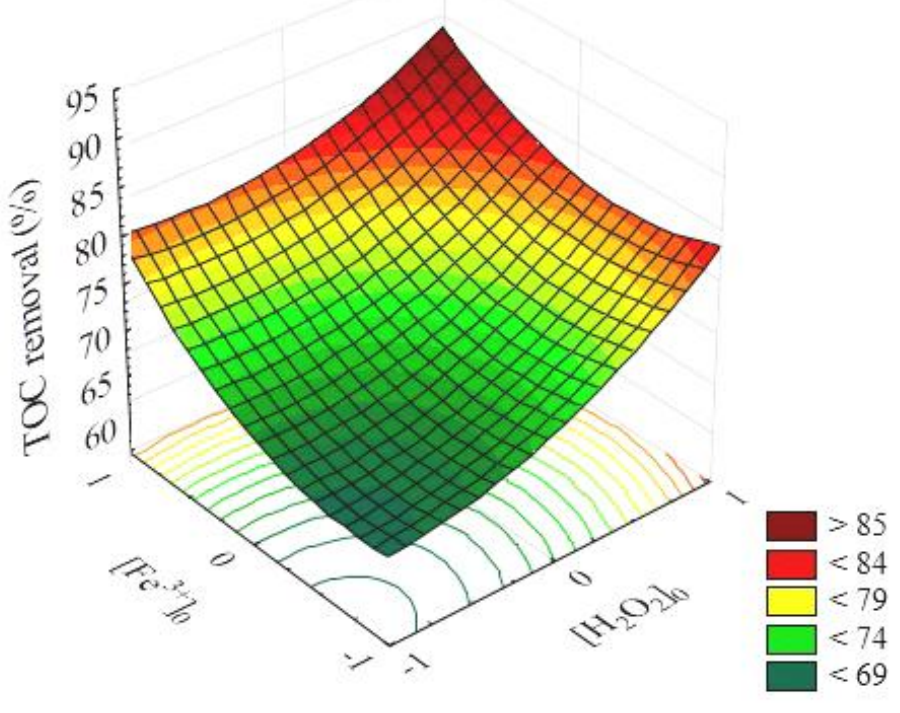

Figure 4.29. Interaction effect between $\left[\mathrm{Fe}^{3+}\right]_{\mathrm{o}}$ and $\left[\mathrm{H}_{2} \mathrm{O}_{2}\right]_{\mathrm{o}}$ on percent TOC removal. 
Table 4.10. Reaction mechanism for complete mineralization of PAA by $\mathrm{UV} / \mathrm{Fe}^{3+} / \mathrm{H}_{2} \mathrm{O}_{2}$.

\begin{tabular}{|c|c|c|c|}
\hline No. & Reaction & Rate Constant & Ref. \\
\hline R1 & $\mathrm{H}_{2} \mathrm{O}_{2} \stackrel{\varphi_{\mathrm{H}_{2} \mathrm{O}_{2}}, h v}{\longrightarrow} 2 \cdot \mathrm{OH}$ & 0.5 mol Einstein ${ }^{-1}$ & Crittenden et al., 1999 \\
\hline R2 & $P A A \stackrel{\varphi_{P A A}, h v}{\longrightarrow} ?$ & 0 & This study \\
\hline R3 & $\mathrm{Fe}^{3+}+\mathrm{H}_{2} \mathrm{O} \stackrel{k_{3}}{\rightarrow} \mathrm{Fe}^{2+}+\cdot \mathrm{OH}+\mathrm{H}^{+}$ & $0.0012 \quad \mathrm{~s}^{-1}$ & Kusic et al., 2009 \\
\hline R4 & $\mathrm{Fe}^{3+}+\mathrm{H}_{2} \mathrm{O}_{2} \stackrel{k_{4}}{\rightarrow} \mathrm{Fe}^{2+}+\mathrm{HO}_{2}^{\cdot}+\mathrm{H}^{+}$ & $0.02 \quad \mathrm{M}^{-1} \mathrm{~s}^{-1}$ & Chen and Pignatello, 1997 \\
\hline R5 & $\mathrm{Fe}^{2+}+\mathrm{H}_{2} \mathrm{O}_{2} \stackrel{k_{5}}{\rightarrow} \mathrm{Fe}^{3+}+{ }^{\cdot} \mathrm{OH}+\mathrm{OH}^{-}$ & $70 \quad \mathrm{M}^{-1} \mathrm{~s}^{-1}$ & Chen and Pignatello, 1997 \\
\hline R6 & $\mathrm{Fe}^{2+}+{ }^{\cdot} \mathrm{OH} \stackrel{k_{6}}{\rightarrow} \mathrm{Fe}^{3+}+\mathrm{OH}^{-}$ & $3.5 \times 10^{8} \mathrm{M}^{-1} \mathrm{~s}^{-1}$ & Chen and Pignatello, 1997 \\
\hline R7 & $\mathrm{Fe}^{3+}+\mathrm{HO}_{2}^{\cdot} \stackrel{k_{7}}{\rightarrow} \mathrm{Fe}^{2+}+\mathrm{O}_{2}+\mathrm{H}^{+}$ & $4 \times 10^{5} \quad \mathrm{M}^{-1} \mathrm{~s}^{-1}$ & Chen and Pignatello, 1997 \\
\hline R8 & $\mathrm{HO}_{2}^{\cdot} \stackrel{k_{8}}{\rightarrow} \mathrm{O}_{2}^{--}+H^{+}$ & $1.6 \times 10^{5} \mathrm{~s}^{-1}$ & Bielski et al., 1985 \\
\hline R9 & $\mathrm{Fe}^{3+}+\mathrm{O}_{2}^{--} \stackrel{k_{9}}{\rightarrow} \mathrm{Fe}^{2+}+\mathrm{O}_{2}$ & $\mathrm{M}^{-1} \mathrm{~s}^{-1}$ & Chen and Pignatello, 1997 \\
\hline R10 & $\mathrm{Fe}^{2+}+\mathrm{O}_{2}^{--} \stackrel{k_{10}}{\longrightarrow} \mathrm{Fe}^{3+}+\mathrm{H}_{2} \mathrm{O}_{2}$ & $1 \times 10^{7}$ & Chen and Pignatello, 1997 \\
\hline R11 & $\mathrm{H}_{2} \mathrm{O}_{2}+\cdot \mathrm{OH}^{k_{11}} \longrightarrow \mathrm{H}_{2} \mathrm{O}+\mathrm{HO}_{2}^{\cdot}$ & $2 \times 10^{7} \quad \mathrm{M}^{-1} \mathrm{~s}^{-1}$ & Buxton et al., 1988 \\
\hline R12 & $\mathrm{HO}_{2}^{-}+\cdot \mathrm{OH}^{k_{12}} \rightarrow \mathrm{HO}_{2}^{\cdot}+\mathrm{OH}^{-}$ & $7 \times 10^{9} \quad \mathrm{M}^{-1} \mathrm{~s}^{-1}$ & Christensen et al., 1982 \\
\hline R13 & $\mathrm{H}_{2} \mathrm{O}_{2}+\mathrm{HO}_{2} \stackrel{k_{13}}{\rightarrow} \mathrm{H}_{2} \mathrm{O}+\cdot \mathrm{OH}+\mathrm{O}_{2}$ & $M^{-1} s^{-1}$ & Koppenol et al., 1978 \\
\hline R14 & $\mathrm{H}^{+}+\mathrm{O}_{2}^{\cdot-} \stackrel{k_{14}}{\rightarrow} \mathrm{HO}_{2}^{\cdot}$ & $1 \times 10^{10} \quad \mathrm{M}^{-1} \mathrm{~s}^{-1}$ & Bielski et al., 1985 \\
\hline R15 & $\cdot \mathrm{OH}+\cdot \mathrm{OH}^{k_{15}} \rightarrow \mathrm{H}_{2} \mathrm{O}_{2}$ & $5.5 \times 10^{9} \quad \mathrm{M}^{-1} \mathrm{~s}^{-1}$ & Bielski et al., 1985 \\
\hline R16 & $\mathrm{HO}_{2}^{\cdot}+\mathrm{HO}_{2} \stackrel{k_{16}}{\rightarrow} \mathrm{H}_{2} \mathrm{O}_{2}+\mathrm{O}_{2}$ & $8.3 \times 10^{5} \quad \mathrm{M}^{-1} \mathrm{~s}^{-1}$ & Bielski et al., 1985 \\
\hline R17 & $\mathrm{HO}_{2}^{\cdot}+\cdot \mathrm{OH}^{k_{17}} \rightarrow \mathrm{H}_{2} \mathrm{O}+\mathrm{O}_{2}$ & $6.6 \times 10^{9} \quad \mathrm{M}^{-1} \mathrm{~s}^{-1}$ & Elliot and Buxton, 1992 \\
\hline R18 & $\mathrm{HO}_{2}^{\cdot}+\mathrm{O}_{2}^{--} \stackrel{k_{18}}{\rightarrow} \mathrm{HO}_{2}^{-}+\mathrm{O}_{2}$ & $9.7 \times 10^{7} \mathrm{M}^{-1} \mathrm{~s}^{-1}$ & Bielski et al., 1985 \\
\hline R19 & $\cdot \mathrm{OH}+\mathrm{O}_{2}^{--} \stackrel{k_{19}}{\rightarrow} \mathrm{OH}^{-}+\mathrm{O}_{2}$ & $8 \times 10^{9} \quad M^{-1} \mathrm{~s}^{-1}$ & Linden et al., 2005 \\
\hline R20 & $\mathrm{H}_{2} \mathrm{O}_{2} \leftrightarrow H^{+}+\mathrm{HO}_{2}^{-}$ & $\mathrm{pK}_{\mathrm{a}}=11.6$ & Perry et al., 1981 \\
\hline R21 & $\mathrm{Cl}^{-}+\cdot \mathrm{OH}^{k_{21}} \rightarrow \mathrm{ClOH}^{\cdot-}$ & $4.2 \times 10^{9} \quad \mathrm{M}^{-1} \mathrm{~s}^{-1}$ & De Laat and Le, 2006 \\
\hline R22 & $\mathrm{ClOH}^{\cdot-} \stackrel{k_{22}}{\rightarrow} \mathrm{Cl}^{-}+\cdot \mathrm{OH}$ & $6 \times 10^{9} \quad \mathrm{M}^{-1} \mathrm{~s}^{-1}$ & De Laat and Le, 2006 \\
\hline R23 & $\mathrm{ClOH}^{\bullet-}+\mathrm{H}^{+} \stackrel{k_{23}}{\longrightarrow} \mathrm{Cl}^{\bullet}+\mathrm{H}_{2} \mathrm{O}$ & $2.4 \times 10^{10} \mathrm{M}^{-1} \mathrm{~s}^{-1}$ & De Laat and Le, 2006 \\
\hline R24 & $\mathrm{Cl}^{\bullet}+\mathrm{H}_{2} \mathrm{O} \stackrel{k_{24}}{\longrightarrow} \mathrm{ClOH}^{\cdot-}+\mathrm{H}^{+}$ & $1 \times 10^{5} \quad \mathrm{M}^{-1} \mathrm{~s}^{-1}$ & De Laat and Le, 2006 \\
\hline R25 & $\mathrm{Cl}^{\bullet}+\mathrm{Cl}^{-} \stackrel{k_{25}}{\rightarrow} \mathrm{Cl}_{2}^{\bullet-}$ & $7.8 \times 10^{9} \quad \mathrm{M}^{-1} \mathrm{~s}^{-1}$ & De Laat and Le, 2006 \\
\hline R26 & $\mathrm{Cl}_{2}^{--} \stackrel{k_{26}}{\rightarrow} \mathrm{Cl}^{\bullet}+\mathrm{Cl}^{-}$ & $5.7 \times 10^{5} \quad \mathrm{M}^{-1} \mathrm{~s}^{-1}$ & De Laat and Le, 2006 \\
\hline R27 & $\mathrm{Cl}^{\bullet}+\mathrm{H}_{2} \mathrm{O}_{2} \stackrel{k_{27}}{\longrightarrow} \mathrm{HO}_{2}^{\cdot}+2 \mathrm{Cl}^{-}+\mathrm{H}^{+}$ & $1 \times 10^{9} \quad \mathrm{M}^{-1} \mathrm{~s}^{-1}$ & De Laat and Le, 2006 \\
\hline R28 & $\mathrm{Cl}_{2}^{\cdot-}+\mathrm{H}_{2} \mathrm{O} \stackrel{k_{28}}{\longrightarrow} \mathrm{ClOH}^{\cdot-}+\mathrm{H}^{+}+\mathrm{Cl}^{-}$ & $1.3 \times 10^{5} \quad \mathrm{M}^{-1} \mathrm{~s}^{-1}$ & De Laat and Le, 2006 \\
\hline R29 & 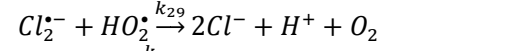 & $3.1 \times 10^{9} \quad \mathrm{M}^{-1} \mathrm{~s}^{-1}$ & De Laat and Le, 2006 \\
\hline R30 & $\mathrm{Cl}_{2}^{--}+\mathrm{O}_{2}^{\cdot-} \stackrel{k_{30}}{\longrightarrow} 2 \mathrm{Cl}^{-}+\mathrm{O}_{2}$ & $2 \times 10^{9} \quad \mathrm{M}^{-1} \mathrm{~s}^{-1}$ & De Laat and Le, 2006 \\
\hline R31 & $\mathrm{Cl}^{\bullet}+\mathrm{Fe}^{2+} \underset{k_{31}}{\longrightarrow} \mathrm{Cl}^{-}+\mathrm{Fe}^{3+}$ & $5.9 \times 10^{9} \mathrm{M}^{-1} \mathrm{~s}^{-1}$ & De Laat and Le, 2006 \\
\hline R32 & $\mathrm{Cl}_{2}^{\bullet-}+\mathrm{Fe}^{2+} \stackrel{32}{\rightarrow} 2 \mathrm{Cl}^{-}+\mathrm{Fe}^{3+}$ & $5 \times 10^{6} \quad \mathrm{M}^{-1} \mathrm{~s}^{-1}$ & De Laat and Le, 2006 \\
\hline R33 & $\mathrm{Fe}^{3+}+\mathrm{Cl}^{-} \stackrel{K_{33}}{\longleftrightarrow} \mathrm{FeCl}^{+}$ & 2.88 & De Laat and Le, 2006 \\
\hline R34 & $\mathrm{Fe}^{3+}+\mathrm{Cl}^{-} \stackrel{K_{34}}{\leftrightarrow} \mathrm{FeCl}^{2+}$ & 6.61 & De Laat and Le, 2006 \\
\hline R35 & $\mathrm{Fe}^{3+}+2 \mathrm{Cl}^{-} \stackrel{K_{35}}{\leftrightarrow} \mathrm{FeCl}_{2}^{+}$ & 10.5 & De Laat and Le, 2006 \\
\hline R36 & $\mathrm{FeCl}^{2+}+\mathrm{HO}_{2}^{\cdot} \stackrel{k_{36}}{\rightarrow} \mathrm{Fe}^{2+}+\mathrm{O}_{2}+\mathrm{H}^{+}+\mathrm{Cl}^{-}$ & $2 \times 10^{4}$ & De Laat and Le, 2006 \\
\hline R37 & $\mathrm{FeCl}_{2}^{+}+\mathrm{HO}_{2} \stackrel{k_{37}}{\longrightarrow} \mathrm{Fe}^{2+}+\mathrm{O}_{2}+\mathrm{H}^{+}+2 \mathrm{Cl}^{-}$ & $2 \times 10^{4}$ & De Laat and Le, 2006 \\
\hline R38 & $\mathrm{FeCl}^{2+}+\mathrm{O}_{2}^{--} \stackrel{k_{38}}{\rightarrow} \mathrm{Fe}^{2+}+\mathrm{O}_{2}+2 \mathrm{Cl}^{-}$ & $5 \times 10^{6}$ & De Laat and Le, 2006 \\
\hline R39 & $\mathrm{P}_{n}+{ }^{\bullet} \mathrm{OH} \stackrel{k_{39}}{\longrightarrow} \mathrm{R}_{n}^{\cdot}+\mathrm{H}_{2} \mathrm{O}$ & $2 \times 10^{8}$ & This study \\
\hline $\mathrm{R} 40$ & $R_{n}^{\cdot} \stackrel{k_{40}}{\longrightarrow} \sum_{\mathrm{n}}\left(R_{n}^{\cdot}-R_{n-1}^{\cdot}\right)+R_{1}^{\cdot}\left(M_{1}\right)$ & $\mathrm{s}^{-1}$ & This study \\
\hline R41 & $\mathrm{M}_{1}+\cdot \mathrm{OH}^{k_{41}} \rightarrow \ldots \rightarrow \mathrm{CO}_{2}+\mathrm{H}_{2} \mathrm{O}$ & $1 \times 10^{6}$ & This study \\
\hline
\end{tabular}


(v) Unlike most kinetic modeling approaches, unsteady state assumptions are used in the free radical rate expressions to have a better prediction of the degradation in the completely-mixed recirculation batch system.

The initiation step in the generalized mechanism is the photolysis of $\mathrm{H}_{2} \mathrm{O}_{2}$ that results in the formation of the highly reactive hydroxyl radical $\left({ }^{\circ} \mathrm{OH}\right)$ that attacks the target compound (PAA) and leads to the chain scission. The hydrogen abstraction of the polymer of chain length $n\left(P_{n}\right)$ results in the generation of the chain-end radical $\left(R_{n}^{*}\right)$ as shown in Reaction (R39). The $\beta$ scission of an end radical leads to the monomer formation $\left(M_{1}\right)$ according to the chain-end scission as presented in Reaction (R40). The monomer degradation results in complete mineralization of the polymer to $\mathrm{H}_{2} \mathrm{O}$ and $\mathrm{CO}_{2}$ (Reaction (R41)) disregarding the intermediates formed. As the polymer degradation is a fragmentation phenomenon, the continuous-distribution mass (population) balances are applied to study the kinetics of the polymer degradation. A free radical mechanism is proposed to evaluate rate coefficients based on the continuous-distribution kinetics which provides the details of the degradation process. The reaction mechanism often used to describe the degradation of macromolecules comprises three reversible elementary steps: initiation-termination of radicals, hydrogen abstraction, and depropagation (Rice and Herzfeld, 1939). According to the long chain approximation (LCA), initiation-termination steps are neglected because of the insignificant effect on the overall reaction rates (Nigam et al., 1994). As shown in Reaction (R39) in Table 4.10, the hydrogen abstraction by hydroxyl radicals from the polymer of the chain length $n$ results in the formation of radicals of the same chain length $\left(R_{n}^{\bullet}\right)$. The binary degradation of $R_{n}^{\bullet}(\mathrm{R} 40)$ can be represented by the following equation in which the integral is used instead of the sum to make the calculations easier: 
$R(n)=\int_{n}^{\infty} k\left(n^{\prime}\right) \Omega\left(n, n^{\prime}\right) r\left(n^{\prime}, t\right) \mathrm{d} n^{\prime}$

where $k\left(n^{\prime}\right)$ and $r\left(n^{\prime}, t\right)$ are the rate constant of the binary scission and the chain length distribution of the polymer radical, respectively. The stoichiometric coefficient $\Omega(n, n)$ represents the likelihood of obtaining a scission product of the chain length $n$ and $n^{\prime}-n$ from a larger molecule $n^{\prime}$. A general expression for the stoichiometric coefficient, or kernel, is given by the following expression (McCoy and Wang, 1994):

$\Omega\left(n, n^{\prime}\right)=\frac{n^{m}\left(n^{\prime}-n\right)^{m} \Gamma(2 m+2)}{\Gamma(m+1)^{2}\left(n^{\prime}\right)^{2 m+1}}$

where $\Gamma$ is the gamma function and $m$ is a parameter indicating the shape of the scission fragment distribution. For a chain-end scission, when a specific product of a fixed chain length 1 and a chain length of $n^{\prime}-1$ are formed, Dirac delta functions are used to represent the stoichiometric kernels, $\Omega\left(n^{\prime}-1, n^{\prime}\right)=\delta\left(n-\left(n^{\prime}-1\right)\right)$ and $\Omega\left(n, n^{\prime}\right)=\delta(n-1)$ (Konaganti and Madras, 2009). The stoichiometric coefficient $\delta\left(n-\left(n^{\prime}-1\right)\right)$ ensures that a product has the chain length of $n=n^{\prime}-1$ and that $n^{\prime} \geq n$ is valid in the interval of integration. Therefore, the mathematical model for the photo-Fenton-like process was developed based on the chemical and photochemical reactions shown in Table 4.10 that was extended with additional reaction steps due to the presence of chloride ion. The developed model includes radical chain reactions taking place during the photo-Fenton-like degradation of PAA in aqueous solution. The primary reaction which initiates the chain reaction is the direct photolysis of $\mathrm{H}_{2} \mathrm{O}_{2}$ to hydroxyl radicals (Reaction R1) as shown in the following equation:

$r_{U V, \mathrm{H}_{2} \mathrm{O}_{2}}=\varphi_{\mathrm{H}_{2} \mathrm{O}_{2}} f_{\mathrm{H}_{2} \mathrm{O}_{2}} I_{o}\left(1-e^{\left(-2.303 b\left(\varepsilon_{\mathrm{H}_{2} \mathrm{O}_{2}}\left[\mathrm{H}_{2} \mathrm{O}_{2}\right]+\varepsilon_{\mathrm{HO}_{2}^{-}}\left[\mathrm{HO}_{2}^{-}\right]+\varepsilon_{T O C_{1}}\left[\mathrm{TOC}_{1}\right]\right)\right)}\right)$ 
where $\varphi_{\mathrm{H}_{2} \mathrm{O}_{2}}$ is the quantum yield of $\mathrm{H}_{2} \mathrm{O}_{2}\left(\right.$ mol Einstein $\left.^{-1}\right), f_{\mathrm{H}_{2} \mathrm{O}_{2}}$ is the fraction of incident light absorbed by $\mathrm{H}_{2} \mathrm{O}_{2}$ and $\mathrm{HO}_{2}^{-}, I_{O}$ is the UV light intensity absorbed by the solution (Einstein $\mathrm{cm}^{-3} \mathrm{~s}^{-}$ ${ }^{1}$ ), $b$ is the effective path length (annular space) in the photoreactor (cm), parameters $\varepsilon_{\mathrm{H}_{2} \mathrm{O}_{2}}, \varepsilon_{\mathrm{HO}_{2}^{-}}$, and $\varepsilon_{T O C_{1}}$ are the molar extinction coefficients of $\mathrm{H}_{2} \mathrm{O}_{2}, \mathrm{HO}_{2}^{-}$, and $\mathrm{TOC}_{1}\left(\mathrm{M}^{-1} \mathrm{~cm}^{-1}\right)$ at $254 \mathrm{~nm}$, respectively, and $\mathrm{TOC}_{1}$ is the total organic carbon concentration of PAA.

The fraction of incident light absorbed by $\mathrm{H}_{2} \mathrm{O}_{2}$ and $\mathrm{HO}_{2}^{-}, f_{\mathrm{H}_{2} \mathrm{O}_{2}}$, could be written by the following equation (Tarr, 2003):

$$
f_{\mathrm{H}_{2} \mathrm{O}_{2}}=\left(\frac{\varepsilon_{\mathrm{H}_{2} \mathrm{O}_{2}}\left[\mathrm{H}_{2} \mathrm{O}_{2}\right]+\varepsilon_{\mathrm{HO}_{2}^{-}}^{-}\left[\mathrm{HO}_{2}^{-}\right]}{\varepsilon_{\mathrm{H}_{2} \mathrm{O}_{2}}\left[\mathrm{H}_{2} \mathrm{O}_{2}\right]+\varepsilon_{\mathrm{HO}}^{-}\left[\mathrm{HO}_{2}^{-}\right]+\varepsilon_{\mathrm{TOC}}\left[\mathrm{TOC}_{1}\right]}\right)
$$

$I_{o}$ which is the incident light at $254 \mathrm{~nm}$ estimated from $14 \mathrm{~W}$ LP lamp over the photoreactor volume assuming $10 \%$ attenuation by the quartz sleeve and 33\% efficiency for the LP lamp. The value of $I_{O}$ was calculated to be $1.97 \times 10^{-8}$ Einstein $\mathrm{cm}^{-3} \mathrm{~s}^{-1}$. Parameters $\varepsilon_{\mathrm{H}_{2} \mathrm{O}_{2}}$ and $\varepsilon_{\mathrm{HO}_{2}^{-}}$are 18.7 $\mathrm{M}^{-1} \mathrm{~cm}^{-1}$ and $210 \mathrm{M}^{-1} \mathrm{~cm}^{-1}$, respectively. The parameter $\varepsilon_{T O C_{1}}\left(320 \mathrm{M}^{-1} \mathrm{~cm}^{-1}\right)$ was determined by measuring the absorbance of several dilutions of PAA in distilled water by means of a spectrophotometer. The absorbance at $254 \mathrm{~nm}\left(A_{254}\right)$ is then related to the extinction coefficient (ع) by the following equation (Ranby and Rabek, 1975):

$A_{254}=\varepsilon_{T O C_{1}} C_{T O C_{1}} l$

where $c_{T O C_{1}}$ and $l$ are the TOC concentration of PAA and the effective path of the UV radiation in the spectrometer $(1 \mathrm{~cm})$, respectively. 
The kinetic expressions (reaction rates) for other reactions involved in the stoichiometric model (Reactions (R3) to (R38) in Table 1) for a set of $j$ reactions and $i$ species could be given by the following Equation:

$R_{j}=k_{j}^{f} \prod_{i \in r e a c t} c_{i}^{-v_{i j}}-k_{j}^{r} \prod_{i \in \operatorname{prod}} c_{i}^{v_{i j}}$

where $k_{j}^{f}$ and $k_{j}^{r}$ denote rate constants for forward and reverse reactions, respectively. The concentration of species $i$ is denoted as $c_{i}$. The stoichiometric coefficients are denoted as $v_{i j}$, which are negative for reactants and positive for products.

Also, the reaction rates of the polymer, polymer radical, and the monomer (Reactions (R39) to (R41)) could be written by the following equations:

$R_{p}(n, t)=-k_{39}(n)\left[{ }^{\bullet} O H\right] p(n, t)$

$R_{r}(n, t)=k_{39}(n)\left[^{\circ} O H\right] p(n, t)-k_{40}(n) r(n, t)+\int_{n}^{\infty} k_{40}\left(n^{\prime}\right) r\left(n^{\prime}, t\right) \delta\left(n-\left(n^{\prime}-1\right)\right) \mathrm{d} n^{\prime}$

$R_{m}(n, t)=\int_{n}^{\infty} k_{40}\left(n^{\prime}\right) r\left(n^{\prime}, t\right) \Omega\left(1, n^{\prime}\right) \mathrm{d} n^{\prime}-k_{41}(n)\left[{ }^{\bullet} O H\right] m(n, t)$

$p(n, t), r(n, t)$, and $m(n, t)$ represent the chain length distribution of the polymer, the chain end radical, and the monomer. At small reaction times, all rate coefficients are usually assumed to be independent of the chain length and they could be considered constant (Madras et al., 1997). In order to make the above differential equations easy to solve, a moment operation is used as follows:

$r^{(p)}(t)=\int_{0}^{\infty} r(n, t) n^{p} \mathrm{~d} n$ 
The zero moment $(p=0)$ is the time-dependent molar concentration of the polymer while the first moment $(p=1)$ is the mass concentration. Therefore, applying the moment operation results in the following ordinary differential equations:

$R_{p^{(p)}}=-k_{39}\left[{ }^{\bullet} \mathrm{OH}\right] p^{(p)}$

$R_{r}^{(p)}=k_{39}\left[{ }^{\bullet} O H\right] p^{(p)}-k_{40} r^{(p)}+k_{40} \sum_{j=0}^{k}(-1)^{j} r^{(k-j)}$

$R_{m}^{(p)}=k_{40} r^{(0)}-k_{41}\left[{ }^{\bullet} O H\right] m^{(p)}$

As mentioned previously, the zero moment represents the molar concentration of the polymer, where the TOC concentration is considered as the output parameter in this study. Therefore, the zero moment is applied to Equations (4.71) to (4.73). $\mathrm{TOC}_{1}$ and $\mathrm{TOC}_{2}$ concentrations are the molar concentrations of polymer and monomer defined as follows:

$$
\begin{aligned}
& R_{\text {OOC }_{1}}=-k_{39}\left[{ }^{\bullet} \mathrm{OH}\right]\left[\mathrm{TOC}_{1}\right] \\
& R_{r^{(0)}}=k_{39}\left[{ }^{\bullet} \mathrm{OH}\right]\left[\mathrm{TOC}_{1}\right] \\
& R_{\text {TOC }_{2}}=k_{40} r^{(0)}-k_{41}\left[{ }^{\bullet} \mathrm{OH}\right]\left[\mathrm{TOC}_{2}\right]
\end{aligned}
$$

Therefore, the obtained dynamic equations that are a set of ordinary differential equations including 23 species and corresponding 41 reaction rates (Table 4.10) were solved simultaneously using the COMSOL Reaction Engineering Lab (Version 3.5) module. To confirm the reliability of the proposed model, the model needs to be validated experimentally, as discussed in the next section. 


\subsubsection{Model Validation}

To verify the reliability of the proposed kinetic model, the model predictions are compared with experimental data obtained by 9 different sets of experiments. The experimental conditions in each set are presented in Table 4.11.

Table 4.11. Experimental conditions of $\mathrm{UV} / \mathrm{H}_{2} \mathrm{O}_{2} / \mathrm{Fe}^{3+}$ process in 9 different sets.

\begin{tabular}{lccccccccc}
\hline Parameter & Exp. & Exp. & Exp. & Exp. & Exp. & Exp. & Exp. & Exp. & Exp. \\
& $\mathbf{1}$ & $\mathbf{2}$ & $\mathbf{3}$ & $\mathbf{4}$ & $\mathbf{5}$ & $\mathbf{6}$ & $\mathbf{7}$ & $\mathbf{8}$ & $\mathbf{9}$ \\
\hline$[\mathrm{PAA}]_{\mathrm{o}} \mathrm{mg}$ & 20 & 40 & 60 & 20 & 40 & 60 & 20 & 40 & 60 \\
{$\left[\mathrm{Fe}^{3+}\right]_{\mathrm{o}} \mathrm{mg}$} & 3 & 5 & 7 & 5 & 3 & 3 & 7 & 7 & 5 \\
{$\left[\mathrm{H}_{2} \mathrm{O}_{2}\right]_{\mathrm{o}} \mathrm{mg}$} & 500 & 900 & 900 & 1300 & 500 & 900 & 1300 & 1300 & 500 \\
\hline $\mathrm{T}=22^{\circ} \mathrm{C}, \mathrm{P}=1 \mathrm{~atm}, \mathrm{pH}=3$, and Flow rate $=3 \mathrm{~L} \mathrm{~min}^{-1}$
\end{tabular}

The critical ranges of the operating conditions were selected based on a set of preliminary experiments. As mentioned earlier, the simulation of the developed model was carried out using COMSOL Reaction Engineering Lab (Version 3.5) module. The results are presented in Figure 4.30, Figure 4.31, and Figure 4.32. As shown in these figures, there is a good agreement between experimental data and the model predictions. The goodness of fit between model predictions and experimental data is shown in Figure 4.33. The high correlation between model predictions and experimental data $\left(R^{2}=0.972\right)$ confirms the satisfactory prediction of the TOC removal as a function of time by the proposed model. Also, it can be concluded from these figures that as it was expected, the lower polymer concentration and the higher dosage of oxidants $\left(\mathrm{H}_{2} \mathrm{O}_{2}\right.$ and $\mathrm{Fe}^{3+}$ ) result in higher percent of TOC removal. Therefore, the optimum operating conditions were achieved at $20 \mathrm{mg} \mathrm{L}^{-1} \mathrm{PAA}, 7 \mathrm{mg} \mathrm{L}^{-1} \mathrm{Fe}^{3+}$, and $1300 \mathrm{mg} \mathrm{L}^{-1} \mathrm{H}_{2} \mathrm{O}_{2}$. 


\subsubsection{Sensitivity Analysis}

The robustness of the mathematical model is estimated by the parametric sensitivity analysis through a systematic procedure. Using the parametric sensitivity analysis, the sensitivity of the model output to input reaction rate constants and consequently the importance of the specific reactions can be determined (Pontes et al., 2010). The system of chemical reactions described by the variable $y$ can be determined by the following differential equations (Varma et al., 1999):

$\frac{d y_{i}}{d t}=f_{i}(y, \phi, t)$

with initial conditions:

$y_{i}(0)=y_{i}^{i}$

where $y$ is the dependent variable, $t$ is the time, and $\phi$ is the vector containing $m$ system input parameters. In this study, $y$ is the TOC concentration of the polymer and $\phi$ is the vector of reaction rate constants. The unique solution for Equation (4.77), which is continuous in $t$ and $\phi$, can be represented by the following equation.

$y_{i}=y_{i}(t, \phi)$

The sensitivity of the dependent variable $y$ with respect to the input parameter $\phi_{j}, s\left(y ; \phi_{j}\right)$, can be defined as follows.

$s\left(y_{i} ; \phi_{j}\right)=\frac{\partial y_{i}\left(t, \phi_{j}\right)}{\partial \phi_{j}}$

The normalized sensitivity of $y$ with respect to $\phi_{j}, S\left(y ; \phi_{j}\right)$ that is generally used in sensitivity analysis can be expressed by the following equation: 


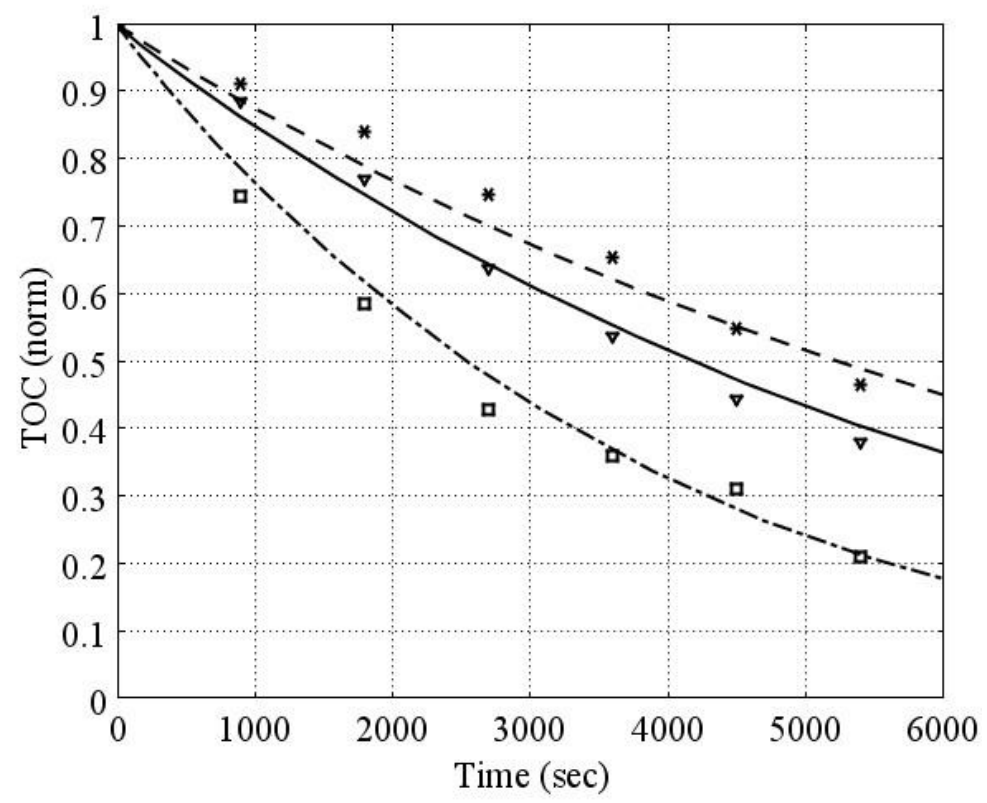

Figure 4.30. Simulation of the proposed kinetic model and comparison with experimental data shown in Table 4.11: Exp.1 ( $\square)$, Exp.2 ( $\nabla)$, and Exp.3 (*).

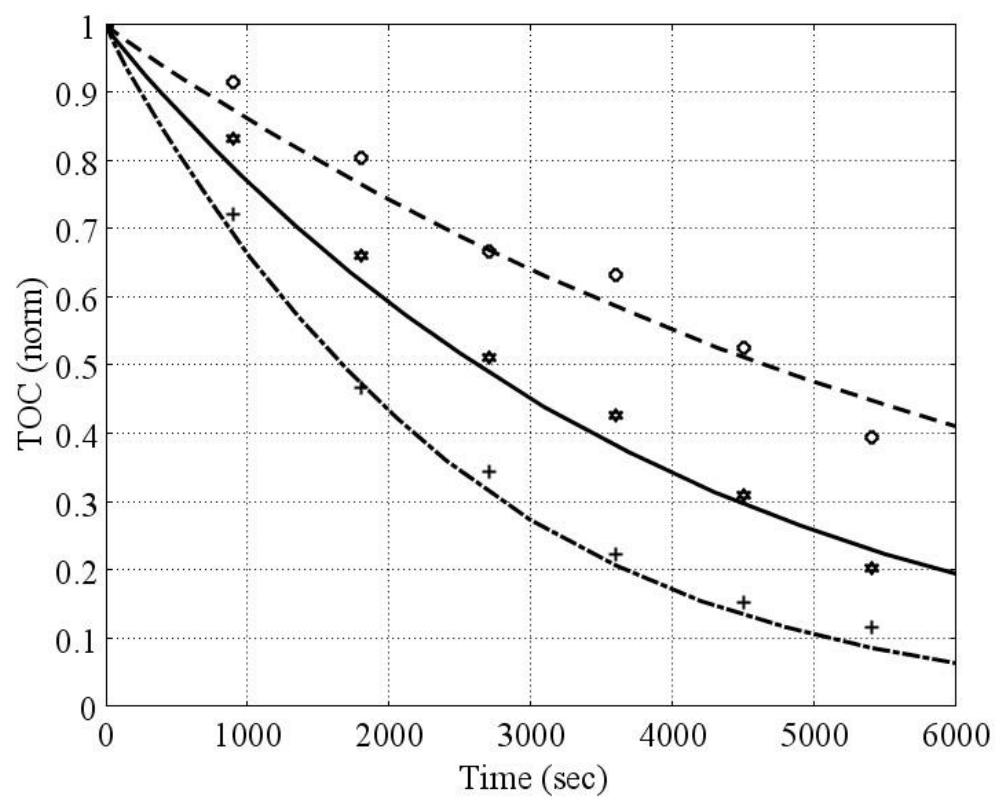

Figure 4.31. Simulation of the proposed kinetic model and comparison with experimental data shown in Table 4.11: Exp.4 (+), Exp.5 (\$), Exp.6 (o). 


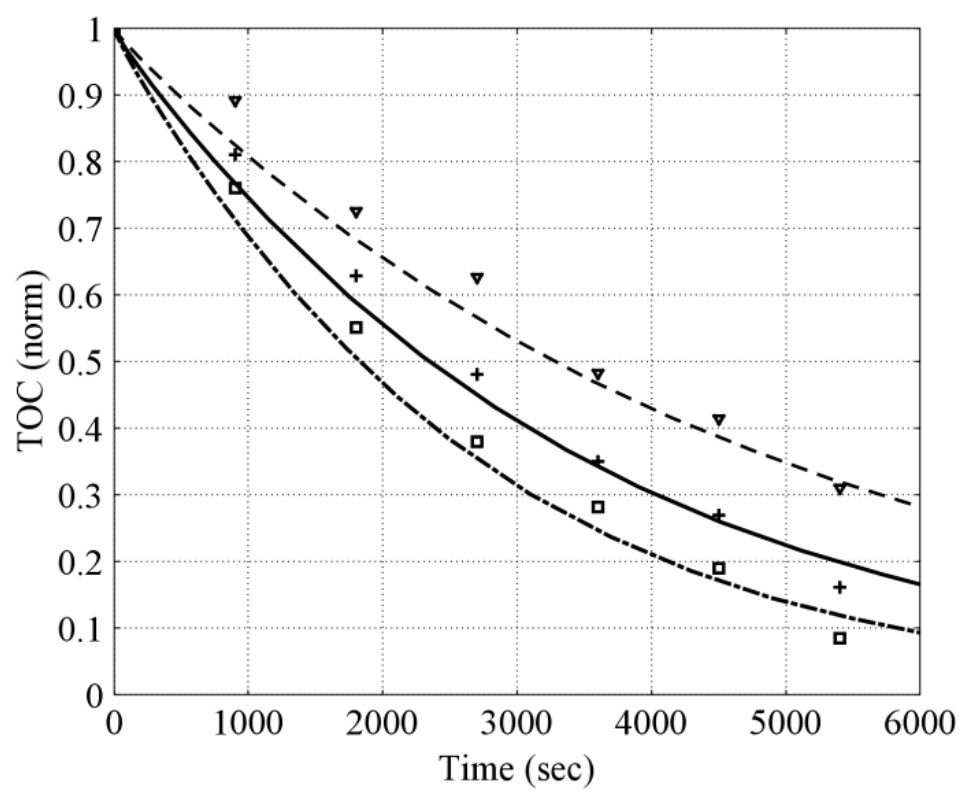

Figure 4.32. Simulation of the proposed kinetic model and comparison with experimental data shown in Table 4.11: Exp.7 ( $\square)$, Exp.8 (+), Exp.9 ( $\nabla)$.

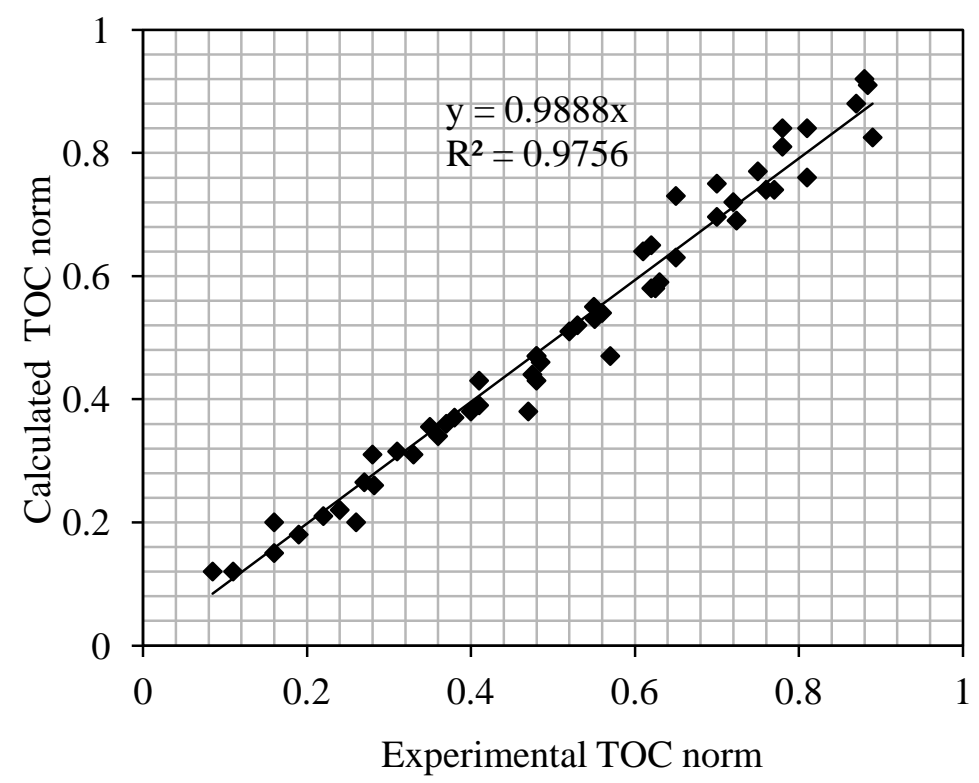

Figure 4.33. Comparison of model predictions with experimental data. 
$s\left(y_{i} ; \phi_{j}\right)=\frac{\phi_{j}}{y} \cdot s\left(y_{i} ; \phi_{j}\right)$

For the kinetic system in this study, the following equation represents a system of ordinary differential equations based on the mass balance that must be solved simultaneously (Varma et al., 1999).

$\frac{d s_{j}}{d t}=J(t) s_{j}+\frac{\partial f(t)}{\partial \phi_{j}}$

with the following initial conditions:

$\left.s_{i}\left(y_{i} ; \phi_{j}\right)\right|_{t=0}= \begin{cases}0, & \phi_{j} \neq y_{j}^{i} \\ 1, & \phi_{j}=y_{j}^{i}\end{cases}$

where

$J(t)=\frac{\partial f}{\partial y}=\left[\begin{array}{cccc}\frac{\partial f_{1}}{\partial y_{1}} & \frac{\partial f_{1}}{\partial y_{2}} & \cdots & \frac{\partial f_{1}}{\partial y_{n}} \\ \frac{\partial f_{2}}{\partial y_{1}} & \frac{\partial f_{2}}{\partial y_{2}} & \cdots & \frac{\partial f_{2}}{\partial y_{n}} \\ \vdots & \vdots & \ddots & \vdots \\ \frac{\partial f_{n}}{\partial y_{1}} & \frac{\partial f_{n}}{\partial y_{2}} & \cdots & \frac{\partial f_{n}}{\partial y_{n}}\end{array}\right]$

and

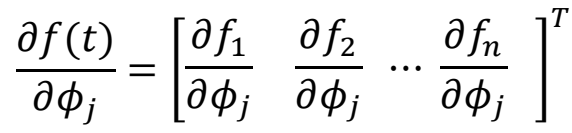

In this study, the dependent variable is considered as the TOC concentration of the polymer at time 90 min (highest TOC removal) with respect to the reaction rate constants shown in Table 4.10. Figure 4.34 represents the peak values of the normalized sensitivity analysis for the TOC concentration with respect to the rate constants of the most important reactions used in the 
kinetic modeling. In this figure, only the reactions with sensitivity coefficients within 2-3 orders of magnitude of the most sensitive reactions are presented. Positive results of the normalized sensitivity coefficient indicate that if a particular reaction rate constant was increased, a lower TOC removal would occur, i.e., the rate of mineralization would be lower (Varma et al., 1999; Kusic et al., 2009). The negative values of the normalized sensitivity coefficient specify higher mineralization rate with an increase in the reaction rate. In fact, the sensitivity analysis illustrates the significance of the specific reaction by showing the magnitude of the increase or decrease in the TOC removal rate. The value of the normalized sensitivity coefficients shows the order of magnitude of change in TOC removal with respect to the change in the given reaction rate by an order of magnitude. As illustrated in Figure 4.34, the most important reaction is Reaction (R39) showing the degradation of the polymer to the polymer radical. That means that an increase in the rate of Reaction (R39) results in the higher TOC removal. The next important reaction is Reaction (R1) that shows the direct photolysis of $\mathrm{H}_{2} \mathrm{O}_{2}$ to the ${ }^{\circ} \mathrm{OH}$. The negative value of the sensitivity analysis for this reaction shows that the increase in the rate of the reaction leads to higher percent of TOC removal.

In Reaction (R1), hydroxyl radicals are generated and they are the only species responsible for the attacking to the polymer backbone. Consequently, the higher rate of ${ }^{\circ} \mathrm{OH}$ generation leads to the higher percent of the TOC removal. Other important reactions according to the sensitivity analysis are Reactions (R40), (R11), (R5), and (R15) as is evident in Figure 4.34. However, these these reactions have values of the normalized sensitivity analysis for 2 order of magnitude less than those in Reactions (R39) and (R1) indicating their minor effect on the percent TOC removal. 


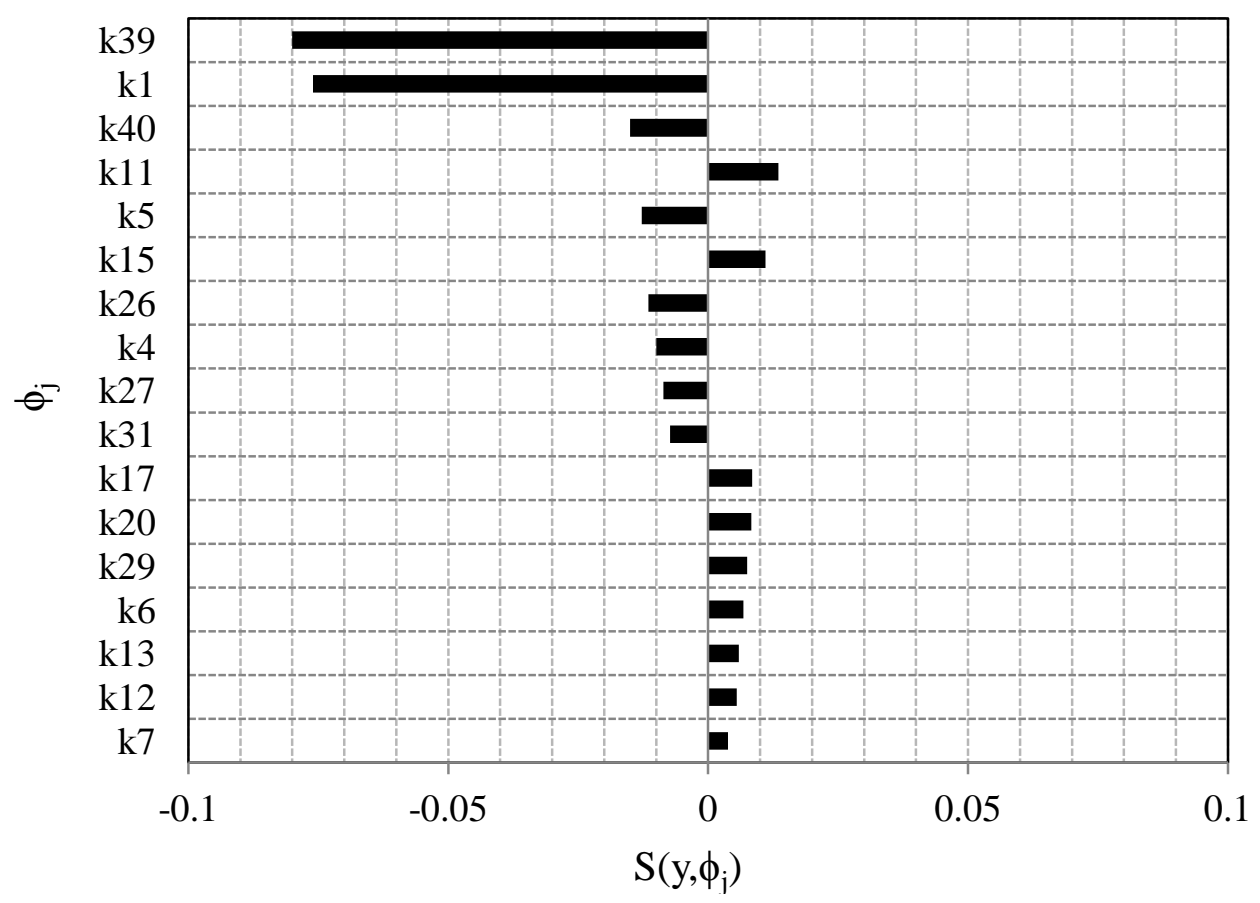

Figure 4.34. The peak values of the normalized sensitivity coefficients for TOC concentration with respect to reaction rate constant for the most important chemical reactions in Table 4.10. 
The negative values of the sensitivity coefficients for Reactions (R40) and (R5) show the positive influence of the higher reaction rates of these two reactions on the percent TOC removal and vice versa for the Reactions (R11) and (R15). The scavenging effect of $\mathrm{H}_{2} \mathrm{O}_{2}$ towards ${ }^{\circ} \mathrm{OH}$ in Reaction (R11) results in the negative effect of this reaction on the percent TOC removal. The recombination of ${ }^{\circ} \mathrm{OH}$ radicals also has negative influence on the percent TOC removal. Other important reactions are shown in Figure 4.34.

\subsubsection{CFD Model Development}

While numerous studies have been conducted in modeling AOTs, there are only limited studies on the CFD modeling for fundamental understanding of the complex photochemical processes. The CFD, as a numerical technique, solves the fluid dynamic equations through space and time including the conservations of mass, momentum, and energy (radiation). These governing equations combined with proper boundary and initial conditions can illustrate physical and chemical changes within a photoreactor. The continuous flow cylindrical photoreactor composed of two concentric cylinders with annular laminar flow ( $\operatorname{Re~53)}$ is shown in Figure 4.35. The inner cylinder is a quartz sleeve in which the UV lamp is inserted. The photoreactor description and operating conditions for the continuous flow in the photoreactor are summarized in Table 4.12. The operating conditions were based on the optimal conditions achieved in 9 sets of experiments as shown in Table 4.11. The main assumptions in the modeling are: (i) steady-state condition, (ii) laminar flow, (iii) incompressible fluid, (iv) isothermal condition $\left(22^{\circ} \mathrm{C}\right)$, and (v) constant physical properties of the solution similar to water. 


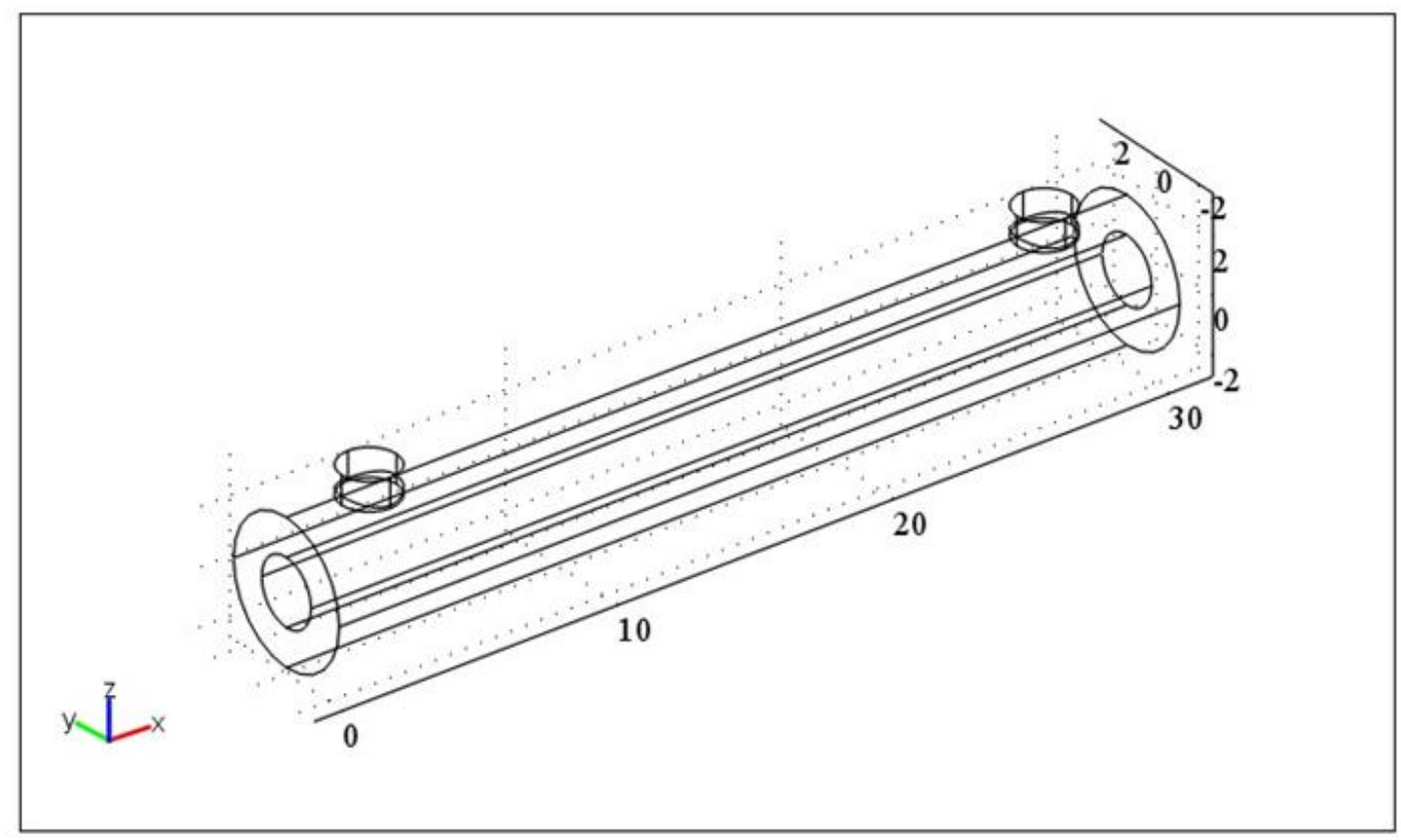

Figure 4.35. Laboratory-scale photoreactor geometry (dimensions are given in centimeters). 
Table 4.12. Laboratory-scale photoreactor description in continuous flow.

\begin{tabular}{llc}
\hline Item & & Specification \\
\hline Photoreactor & Type & Annular \\
& Inner Diameter & $2.44 \mathrm{~cm}$ \\
& Outer Diameter & $5.11 \mathrm{~cm}$ \\
& Length & $30.5 \mathrm{~cm}$ \\
& Volume & $0.46 \mathrm{~L}$ \\
Lamp & Type & LP Hg UV \\
& Wavelength & $254 \mathrm{~nm}$ \\
& Length & $20.5 \mathrm{~cm}$ \\
Temperature & Output power & $4 \mathrm{~W}$ \\
Pressure & & $22^{\circ} \mathrm{C}$ \\
Operating Flow Rate & & $1 \mathrm{~atm}$ \\
$\mathrm{Re}_{\left[\mathrm{PAA}_{\text {in }}\right.}$ & & $0.1-0.5 \mathrm{~L} \mathrm{~min}^{-1}$ \\
{$\left[\mathrm{H}_{2} \mathrm{O}_{2}\right]_{\text {in }}$} & & 53 \\
{$\left[\mathrm{Fe}^{3+}\right]_{\text {in }}$} & & $20 \mathrm{mg} \mathrm{L}^{-1}$ \\
\hline
\end{tabular}

To simulate the 3D fluid field within the cylindrical photoreactor, COMSOL Multiphysics (Version 3.5) was utilized. This commercial package is based on the finite element method used for CFD modeling. The photoreactor modeling requires transport-reaction coupling which exists in COMSOL Multiphysics Chemical Engineering Module and Reaction Engineering Lab. The COMSOL Reaction Engineering Lab is a tool that uses reaction formulas (kinetic expressions) to create models involving momentum, mass, and energy in COMSOL Multiphysics. The main step in CFD modeling is to discretize the flow domain within the photoreactor to small elements which is also referred to grid generation. Generally, the density of cells has to be fine enough to achieve the flow details. In order to determine the number of cells for discretization and to evaluate the accuracy of the numerical results, the grid independence studies were carried out. The grid convergence criteria (10,427 elements) were set as the additional cells did not change the velocity magnitude and calculated concentration by more than $3 \%$ for all grid points. 
Therefore, the CFD modeling of photo-Fenton-like process requires simultaneous solving of the momentum, mass, and energy balances.

\subsubsection{Momentum balance}

For the laminar flow (Re 53), the steady-state incompressible Navier-Stokes equations is used for the momentum balance. Therefore, the governing equations for continuity and the momentum balances at constant density and viscosity could be described as follows (Bird et al., 2002):

$\nabla \cdot u=0$

$\rho(u \cdot \nabla) u=\nabla \cdot\left[-p I+\eta\left(\nabla u+(\nabla u)^{T}\right)\right]+F$

where $\rho, u, p, \eta$, and $F$ are density, velocity vector, pressure, dynamic viscosity, and the body force vector. The boundary conditions for the inlet is the known value of the velocity, the zero normal stress for the outlet, and no slip boundary conditions for all walls of the photoreactor and quartz sleeve. The velocity profile is achieved by simultaneous solving of continuity and momentum equations along with boundary conditions. The velocity profile is presented in Figure 4.36. As depicted in Figure 4.36, the velocity near the walls of the photoreactor and quartz sleeve is close to zero as it was expected because of the no slip boundary condition. Also, as is evident in Figure 4.36 (a) and 4.36 (b), the velocities in the inlet and the outlet of the photoreactor are higher due to the smaller cross sectional area.

\subsubsection{Mass balance}

The continuity equation for steady-state mass balance of each species could be written as follows: 
$\nabla \cdot\left(-D \nabla C_{i}\right)=-u \cdot \nabla C_{i}+R_{i}$

where $D$ is the diffusion coefficient, $C_{i}$ is the concentration of species $i, u$ is the velocity vector, and $R_{i}$ is the reaction rate of species $i$. This governing equation is transport-reaction coupling which is carried out by directly exporting COMSOL Reaction Engineering Lab, including the reaction formulas for all species present in Table 4.12, to the COMSOL Multiphysics Chemical Engineering Module to create the mass balance. The boundary conditions for the mass balance are the inlet concentration of the species. There are only four species $\left(\mathrm{PAA}, \mathrm{H}_{2} \mathrm{O}_{2}, \mathrm{Fe}^{3+}\right.$, and $\mathrm{Cl}^{-}$) that have non-zero concentration at the inlet. The convective flux is considered at the outlet for all species. The concentration gradients on the photoreactor walls and the quartz sleeve are zero.

The concentration profile was obtained by solving the governing equation for transport-reaction coupling. The TOC concentration profile is presented in Figure 4.37. As illustrated in this figure, the TOC concentration decreases as the solution passes through the photoreactor. Higher residence time inside the reactor results in higher TOC removal. The experimental TOC removal achieved at the operating conditions in Table 4.12 was $14.18 \%$ that is in good agreement with the CFD simulation result (13.09\%). The concentration profiles for $\mathrm{Fe}^{3+}$ and $\mathrm{Fe}^{2+}$ concentrations are also presented in Figure 4.38 and Figure 4.39, respectively. As evident in these figures, the concentration of $\mathrm{Fe}^{3+}$ reduces as the solution passes through the photoreactor and simultaneously the concentration of $\mathrm{Fe}^{2+}$ increases as it can be also seen in the corresponding reactions in Table 4.10 (Reactions (R3) - (R10)). The concentration profile for ${ }^{\circ} \mathrm{OH}$ is also presented in Figure 4.40. 
(a)

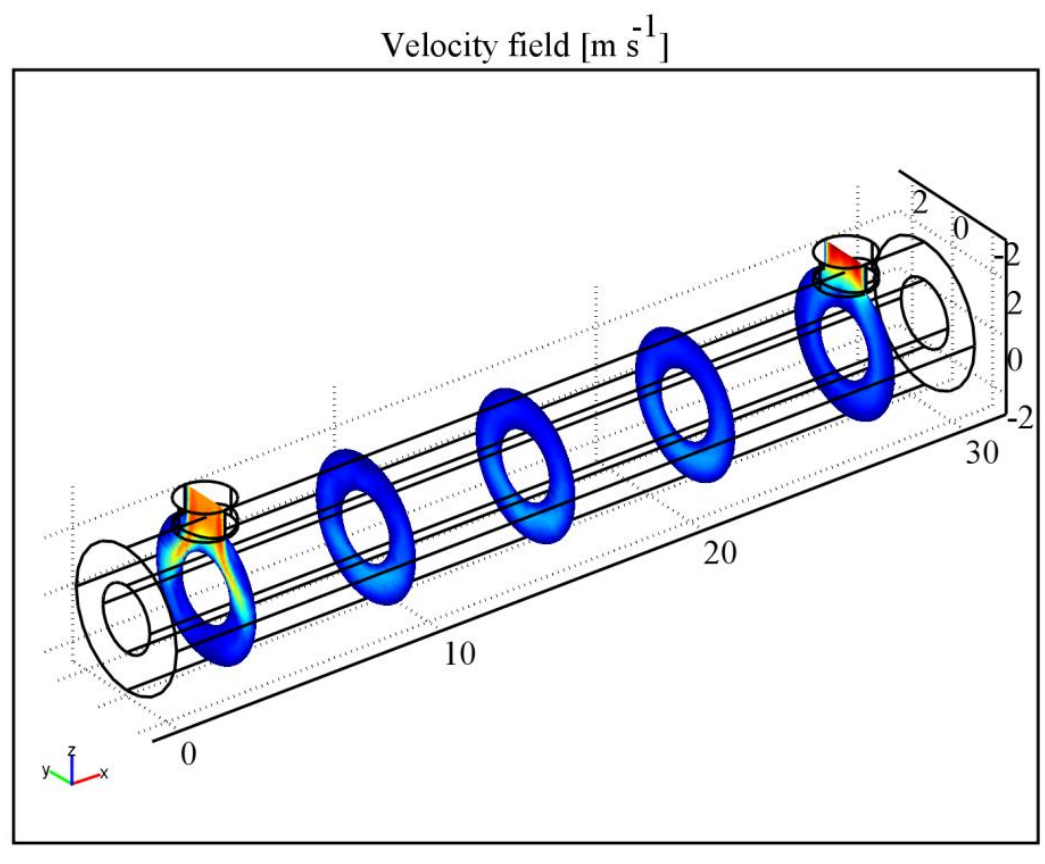

(b)

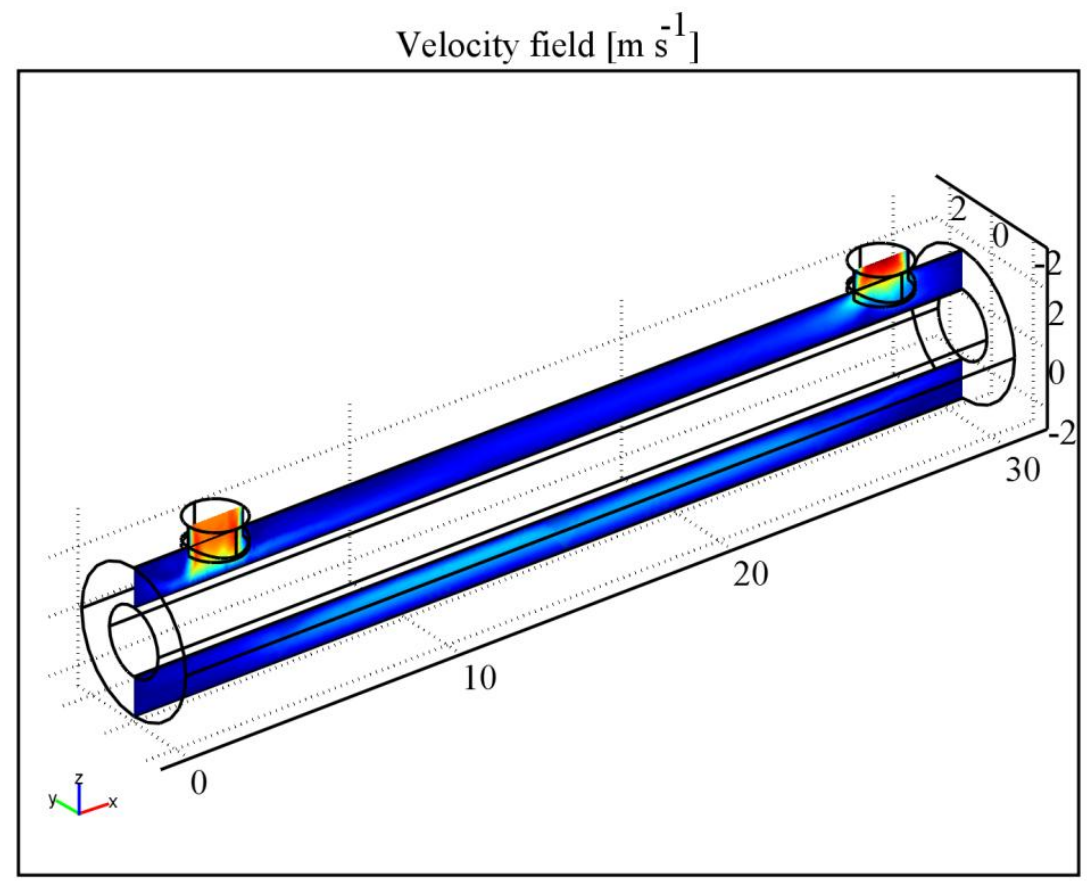

Max: $7.24 \mathrm{e}-3$

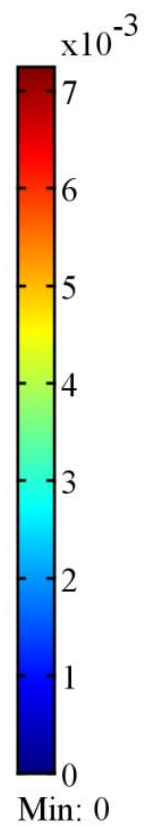

Max: 7.081e-3

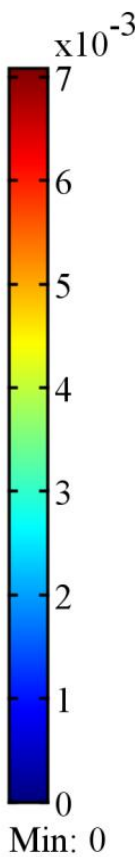

Figure 4.36. CFD simulation for axial velocity in laboratory-scale photoreactor (a) top view (b) side view. 
(a)

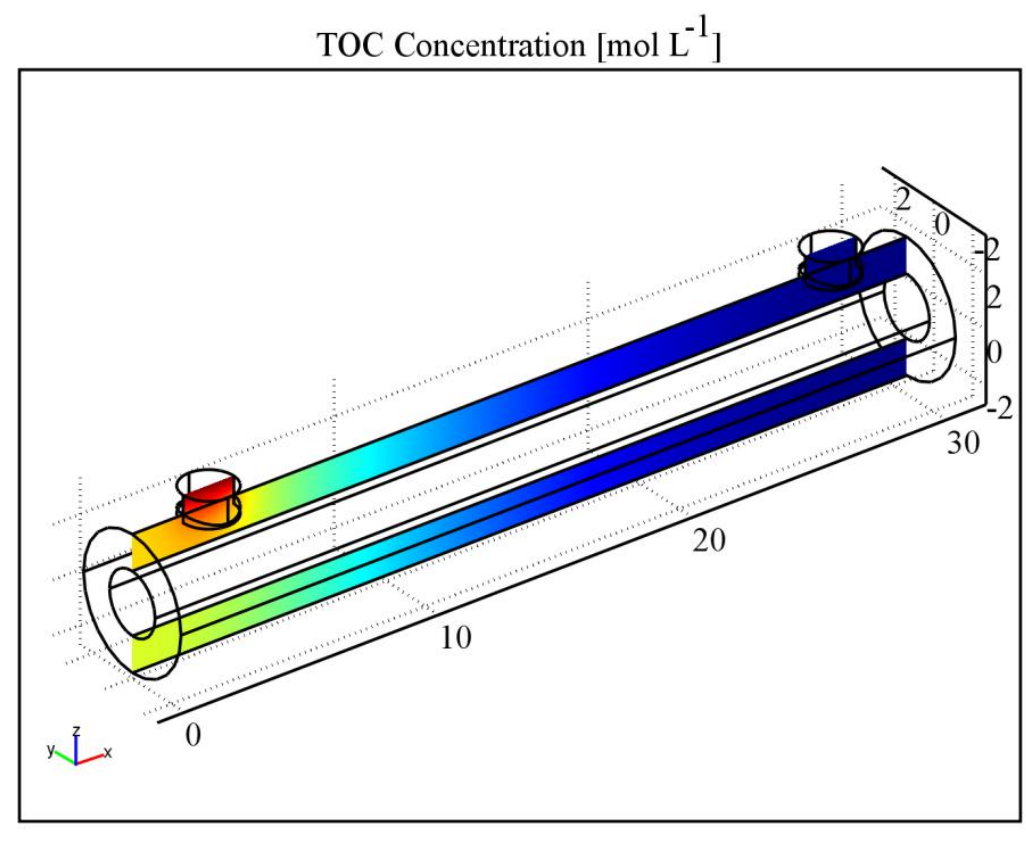

Max: $8.107 \mathrm{e}-4$

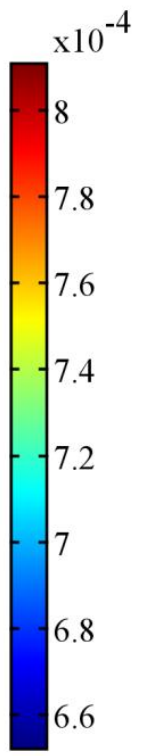

Min: 6.522e-4

(b)

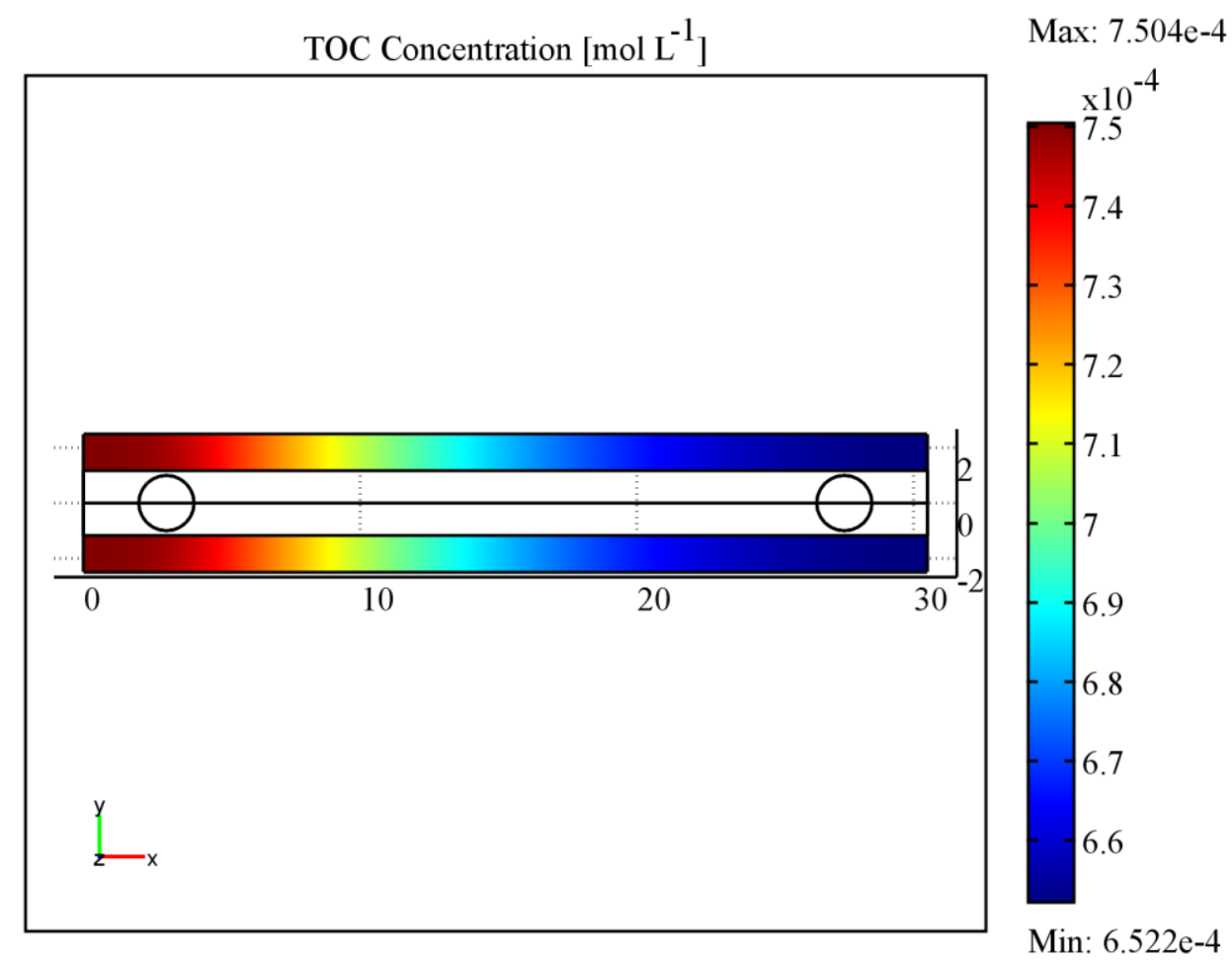

Figure 4.37. CFD simulation for TOC concentration profile in laboratory-scale photoreactor (a) side view (b) top view. 
As shown in this figure, ${ }^{\circ} \mathrm{OH}$ is produced by the direct photolysis of $\mathrm{H}_{2} \mathrm{O}_{2}$ according to the Reaction (R1). High concentration of ${ }^{\circ} \mathrm{OH}$ at the inlet of the photoreactor results in the TOC degradation (Figure 4.37) as ${ }^{\circ} \mathrm{OH}$ is responsible for the attack to the polymer molecules.

\subsubsection{Radiation balance}

In order to simulate the radiation balance, the local volumetric rate of energy absorption (LVREA) per unit volume and unit time must be determined. The LVREA appears in the kinetic expression for direct photolysis of $\mathrm{H}_{2} \mathrm{O}_{2}$ (Reaction (R1) in Table 4.10). The radiation field is not uniform within the photoreactor due to the light attenuation by different species present in the solution. The governing equation for $\operatorname{LVREA}\left(e_{\lambda}^{a}\right)$ from a lamp centered at a point $(a, b)$ within the photoreactor could be given by the following equation (Johnson and Mehrvar, 2008):

$e_{\lambda}^{a}=\mu_{s} I_{0} \frac{r_{i n}}{\sqrt{(x-a)^{2}+(y-b)^{2}}} \exp \left(-2.303 \mu_{s}\left(\sqrt{(x-a)^{2}+(y-b)^{2}}-r_{i n}\right)\right)$

where $\mu_{s}$ is the extinction coefficient of the solution, $I_{o}$ is the radiant energy flux on the sleeve, $x$ and $y$ are Cartesian coordinates, and $r_{i n}$ is the inner radius (radius of the quartz sleeve). The parameter $\mu_{s}$ is a linear function of the concentration of the absorbing species as shown in the following equation.

$\mu_{s}=\sum \varepsilon_{i} C_{i}$

where $\varepsilon_{i}$ is the molar extinction coefficient of each species. The axial local volumetric rate of energy absorption (LVREA) presented in Figure 4.41 indicates that the highest rate of energy absorption is close to the UV lamp where consequently there is the highest generation of ${ }^{\circ} \mathrm{OH}$ (Figure 4.40). Consequently, higher TOC removal would be achieved at higher light intensity, higher residence time, lower photoreactor radius, and higher photoreactor length. 


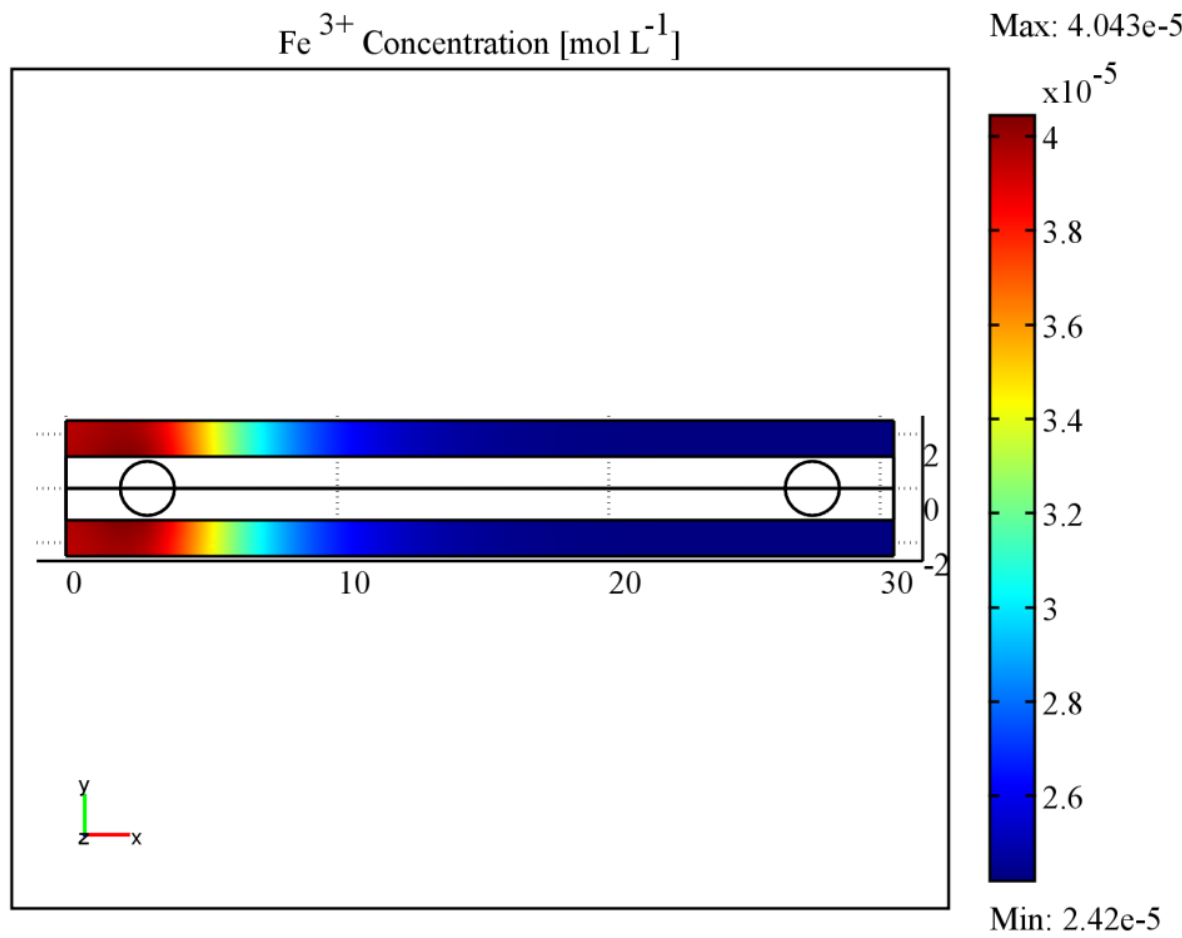

Figure 4.38. CFD simulation for $\mathrm{Fe}^{3+}$ concentration profile in laboratory-scale photoreactor.

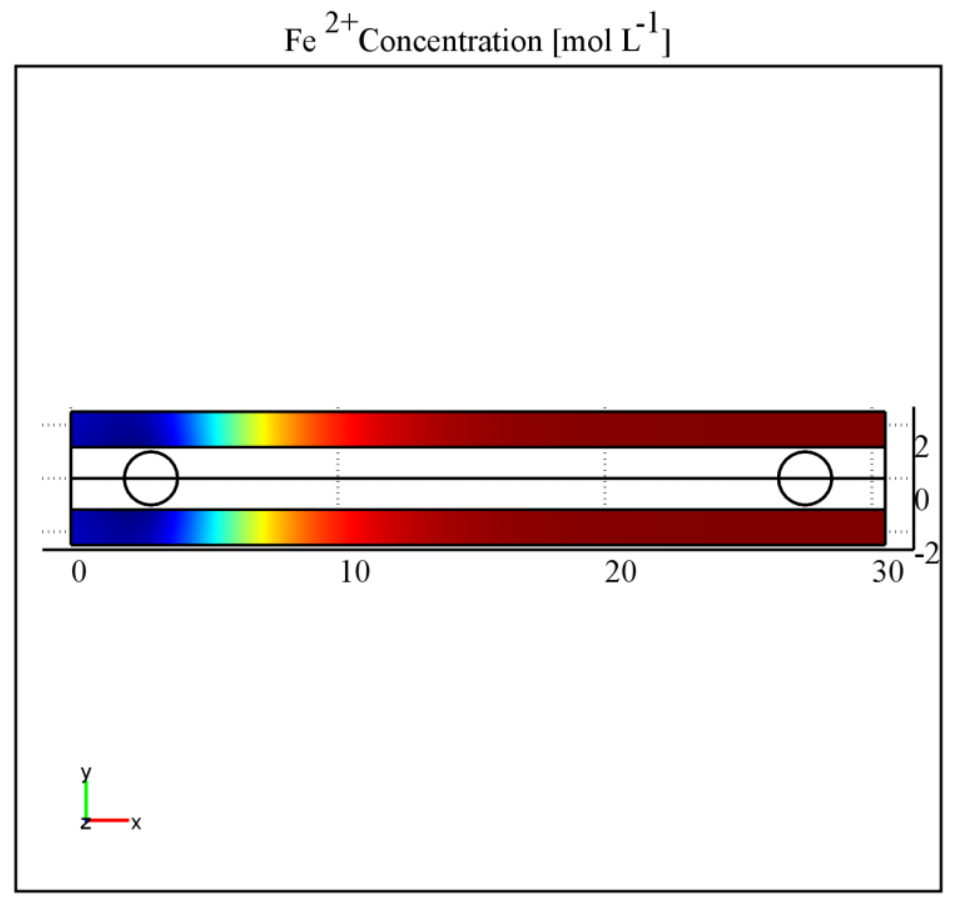

Max: 9.869e-5

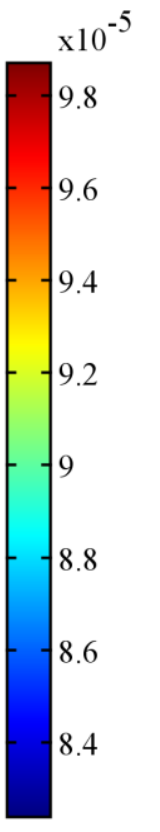

Min: $8.24 \mathrm{e}-5$

Figure 4.39. CFD simulation for $\mathrm{Fe}^{2+}$ concentration profile in laboratory-scale photoreactor. 


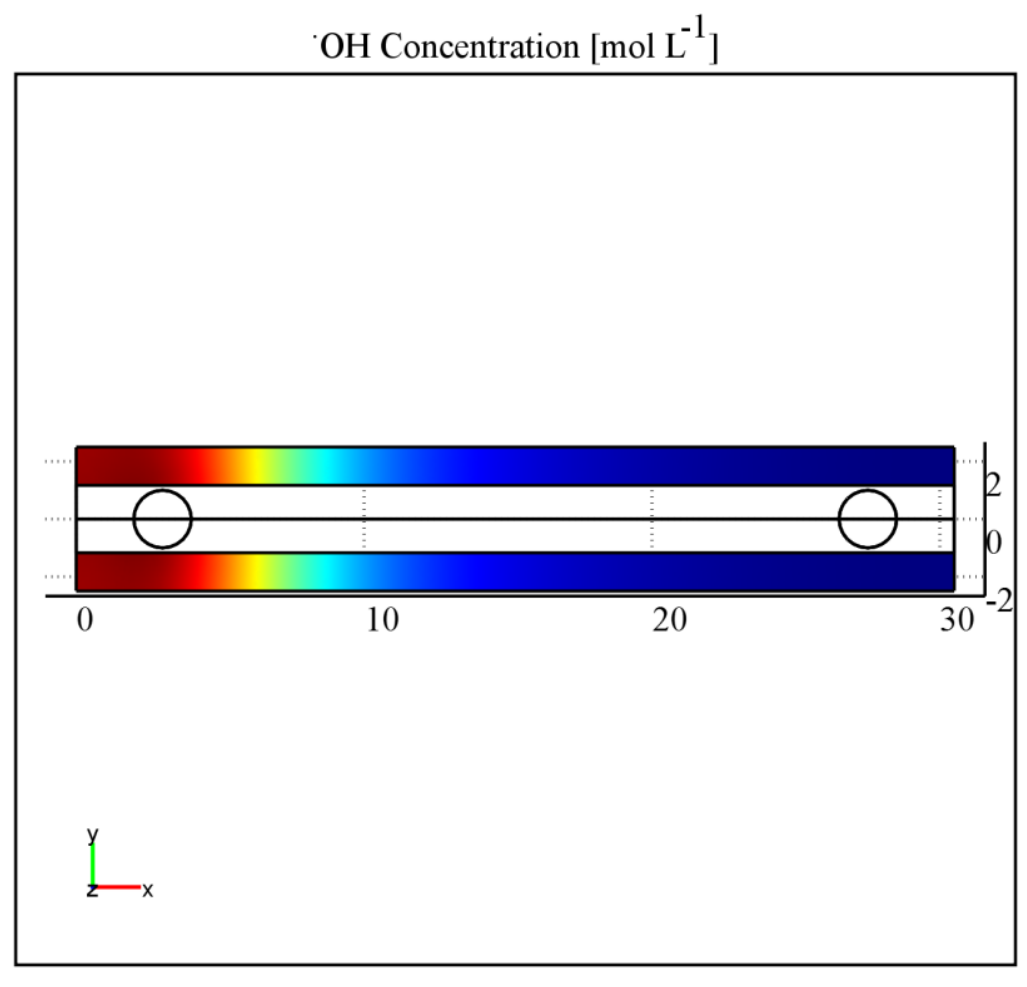

Max: 3.972e-9

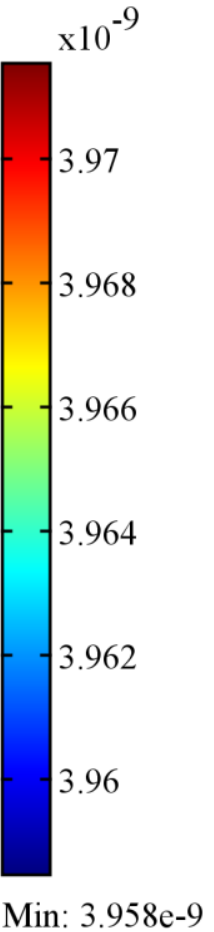

Figure 4.40. CFD simulation for ${ }^{\circ} \mathrm{OH}$ concentration profile in laboratory-scale photoreactor. 
(a)

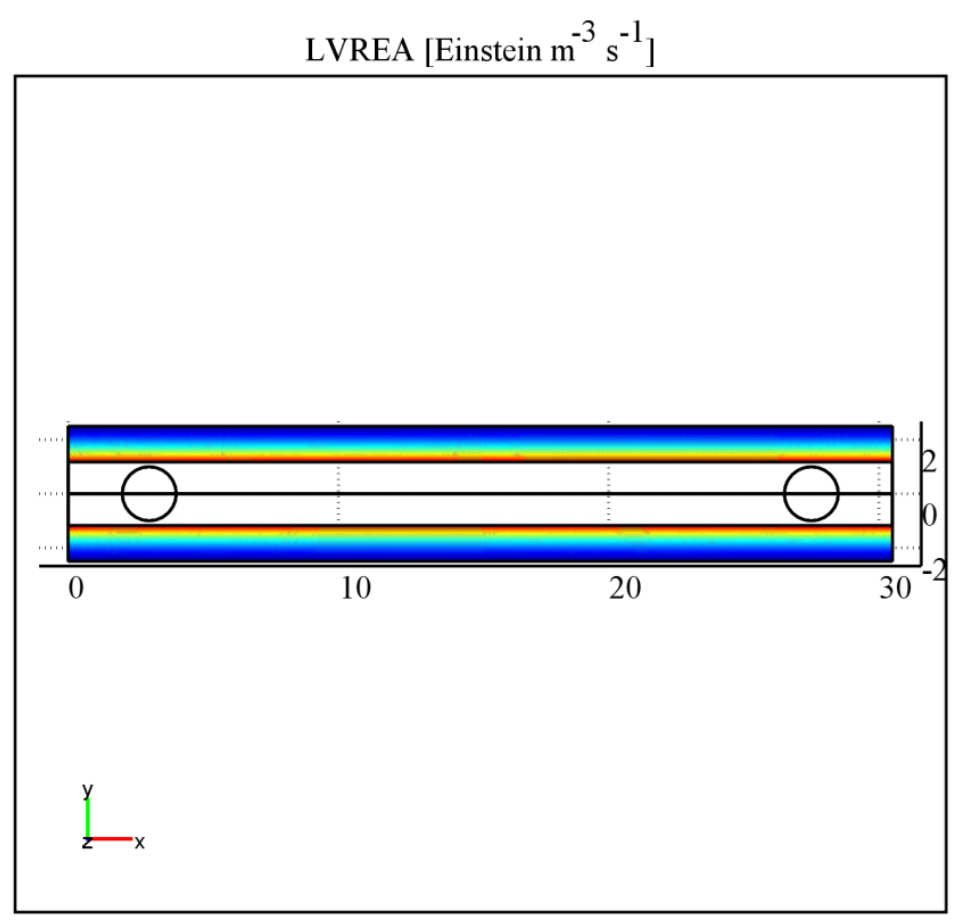

(b)

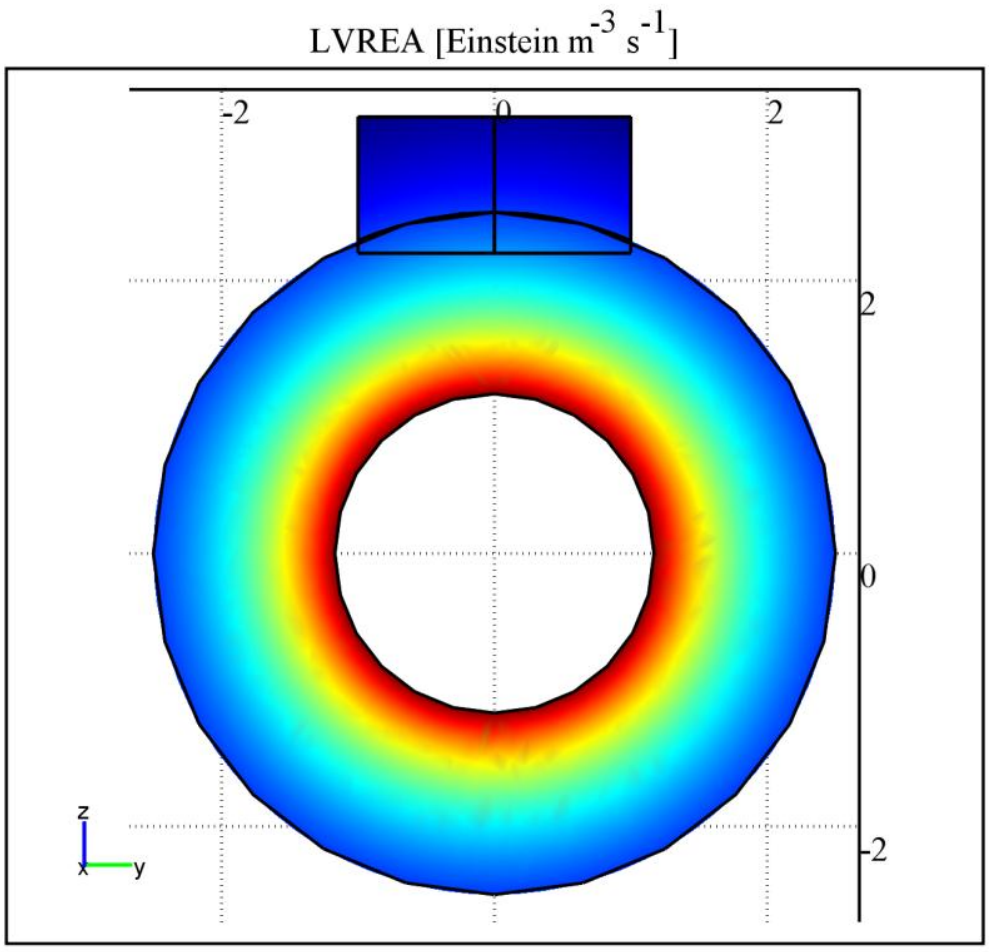

Max: 3.086e-7

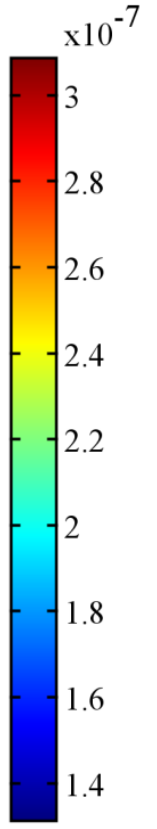

Min: $1.313 \mathrm{e}-7$

Max: 3.096e-7

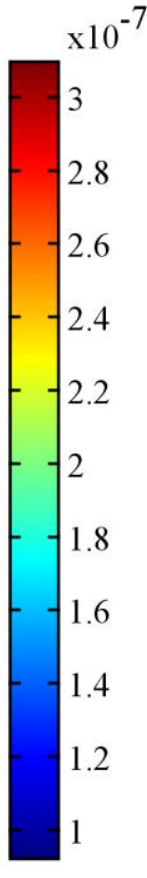

Min: $9.219 e-8$

Figure 4.41. CFD simulation for LVREA in laboratory-scale photoreactor (a) top view (b) cross section. 


\subsubsection{Conclusions}

The application of a three-factor, three-level Box-Behnken experimental design combined with RSM and quadratic programming was investigated for photo-Fenton-like degradation of PAA in aqueous solution. The developed mathematical model provided a critical analysis of the simultaneous interactive effects of the independent variables. The adequacy of the proposed model was tested using various descriptive statistics. The statistical analysis using ANOVA indicated that the developed quadratic models were highly accurate and predictive. A good agreement between quadratic model predictions and observed values also confirmed the accuracy of the developed model. The initial concentration of PAA and the initial $\mathrm{Fe}^{3+}$ and $\mathrm{H}_{2} \mathrm{O}_{2}$ dosage had significant effect on the percent TOC removal. The optimal operating conditions to achieve the maximum percent of TOC removal (93.94\%) in the selected range was determined to be $22.80 \mathrm{mg} \mathrm{L}^{-1} \mathrm{PEO}, 7 \mathrm{mg} \mathrm{L}^{-1} \mathrm{Fe}^{3+}$, and $1300 \mathrm{mg} \mathrm{L}^{-1} \mathrm{H}_{2} \mathrm{O}_{2}$, respectively. Finally, the model prediction for the maximum TOC removal was verified by an additional experimental run at the obtained optimum operating conditions. The validation results clearly confirmed that a threefactor three-level Box-Behnken experimental design combined with RSM and quadratic programming is an effective tool for mathematical modeling of photo-Fenton-like process. In the second part, a detailed mathematical kinetic model for $\mathrm{UV} / \mathrm{Fe}^{3+} / \mathrm{H}_{2} \mathrm{O}_{2}$ process including reactions of advanced oxidation technologies, iron chlorocomplexes, inorganic anion radicals, and polymer cleavage was developed. The local volumetric rate of energy absorption was also taken into account in the proposed model. Continuous distribution kinetics was applied to model the dynamics of the polymer degradation based on the chain-end scission. Six sets of experiments in a batch recirculation system were carried out to validate the proposed model predictions. The goodness of fit between model prediction and experimental data confirmed that the model could 
be a good approach to photo-Fenton-like degradation of water-soluble polymers. A sensitivity analysis was conducted to determine intensive kinetic parameters in the mathematical model. The kinetic parameter of the hydrogen abstraction from the polymer and also the model parameter of the photolysis of hydrogen peroxide were the most influential parameters on the percent TOC removal. Some other reaction rate constants were also of importance. In order to determine spatial distribution of different parameters, CFD modeling incorporating rigorous kinetic rate expressions and governing transport equations were studied in a continuous flow at the optimum operating conditions. CFD model verification for TOC was carried out using experimental data at optimum operating conditions. A good agreement between CFD simulation results and the experimental data was indicative of the model adequacy. 


\subsection{OPTIMIZATION OF PHOTO-FENTON-LIKE DEGRADATION OF AQUEOUS POLYACRYLIC ACID USING TWO PHOTOREACTORS IN SERIES}

\subsubsection{Introduction}

As mentioned in Section 2.1.1.2, photo-Fenton-like process, as one of the AOTs, has received great attention in recent decades due to its capacity in oxidizing and mineralizing recalcitrant compounds that are not amenable to biodegradation (Farias et al., 2009; Grcic et al., 2011). In the photo-Fenton-like process, a powerful non-selective source of oxidation $\left({ }^{\circ} \mathrm{OH}\right)$ is generated from $\mathrm{H}_{2} \mathrm{O}_{2}$ in the presence of $\mathrm{Fe}^{3+}$ ions. The great oxidation potential of these short-lived free radicals (especially ${ }^{\circ} \mathrm{OH}$ ) results in the fast degradation or decomposition of target organic compounds. The hydrogen abstraction from the main chain of the polymer by free radicals formed is the most important reason for the photodegradation of polymers (Shukla et al., 2009). The main advantages of the Fenton reagent are its ability to convert a wide range of pollutants to biodegradable products without environmental threat by its residual reagents, ease of operation, and the relatively low cost of the reagents (Tarr, 2003). The photo-Fenton-like process, similar to other AOTs, is a multifactor system where its efficiency is affected by different parameters such as initial concentrations of organic compounds, $\mathrm{H}_{2} \mathrm{O}_{2}$, and iron, as well as $\mathrm{pH}$ and other operating conditions. Therefore, cross-factor effects must be considered to characterize the process and determine the synergetic effects of the process variables.

In this section, an experimental design for the PAA photodegradation by photo-Fenton-like process is investigated in a batch recirculation system using two photoreactors in series 
(Ghafoori et al., 2013b). PAA, a carbon chain water-soluble polymer with the pendant carboxylic acids known to be recalcitrant to biodegradation, was considered as a model water-soluble polymer. The effects of initial concentrations of PAA and $\mathrm{H}_{2} \mathrm{O}_{2}: \mathrm{Fe}^{3+}$, $\mathrm{pH}$, and the recirculation rate on the percent TOC removal rate and the fitted values of "pseudo"-second order mineralization rate constants were studied using a four-factor three-level Box-Behnken experimental design combined with RSM and quadratic programming.

\subsubsection{Experimental Procedure}

The PAA solution was diluted to achieve the desired PAA concentration in a 4-L solution. The lamp was turned on for $30 \mathrm{~min}$ before the beginning of each experiment to stabilize the light intensity. The desired dosage of oxidizers $\left(\mathrm{H}_{2} \mathrm{O}_{2}\right.$ and $\left.\mathrm{Fe}^{3+}\right)$ was added to the solution at the beginning of each experiment. The solution was fed to the system with two photoreactors in series while the temperature was kept constant at $25 \pm 2^{\circ} \mathrm{C}$ during each experiment by means of a heat exchanger. The samples were taken from the collection tank at time 0 and at a time interval of 30 min during a total reaction time of $120 \mathrm{~min}$. The TOC concentration of the samples was measured by a TOC analyzer (Apollo 9000, Teledyne Tekmar, USA) right after taking the samples.

\subsubsection{Preliminary Experiments}

In order to obtain preliminary insight into TOC removal efficiency, different processes including

$\mathrm{UV}$ alone, $\mathrm{UV} / \mathrm{H}_{2} \mathrm{O}_{2}, \mathrm{UV} / \mathrm{Fe}^{3+}$, and $\mathrm{UV} / \mathrm{H}_{2} \mathrm{O}_{2} / \mathrm{Fe}^{3+}$ for one photoreactor and two photoreactors in series were carried out. In each case, the total organic carbon of the PAA solution was 
determined during the total reaction time of 120 min. Dark experiments did not show any TOC removal after $120 \mathrm{~min}$. The results of TOC removal for these processes operated in recirculating batch mode are presented in Figure 4.42. As illustrated in this figure, the TOC removal efficiency in one single reactor using UV alone was negligible.

Also, the TOC removal efficiency in two photoreactors in series showed only $33 \%$ reduction using UV alone (Figure 4.43). In $\mathrm{UV} / \mathrm{H}_{2} \mathrm{O}_{2}$ process, a drastic improvement in the TOC removal efficiency was observed in both single photoreactor and two photoreactors in series. The TOC reduction by $\mathrm{UV} / \mathrm{H}_{2} \mathrm{O}_{2}$ process was due to the attack of the free radicals (mainly ${ }^{\circ} \mathrm{OH}$ ) to the polymer molecules based on the following summarized reactions:

$$
\begin{aligned}
& \mathrm{H}_{2} \mathrm{O}_{2} \stackrel{h v}{\rightarrow} 2 \cdot \mathrm{OH} \\
& \mathrm{Fe}^{3+}+\mathrm{H}_{2} \mathrm{O}_{2} \rightarrow \mathrm{Fe}^{2+}+\mathrm{HO}_{2}^{\bullet}+\mathrm{H}^{+} \\
& \mathrm{Fe}^{2+}+\mathrm{H}_{2} \mathrm{O}_{2} \rightarrow \mathrm{Fe}^{3+}+\cdot \mathrm{OH}+\mathrm{OH}^{-} \\
& \mathrm{Fe}^{3+}+\mathrm{H}_{2} \mathrm{O} \stackrel{h v}{\rightarrow} \mathrm{Fe}^{2+}+\cdot \mathrm{OH}^{+}+\mathrm{H}^{+} \\
& \mathrm{TOC}+\cdot \mathrm{OH} \rightarrow \ldots \rightarrow \mathrm{CO}_{2}+\mathrm{H}_{2} \mathrm{O}
\end{aligned}
$$

However, the TOC reduction by the $\mathrm{UV} / \mathrm{H}_{2} \mathrm{O}_{2}$ process in two photoreactors in series was higher because of the higher light intensity resulting in the generation of more free radicals (Reaction (4.91)). As illustrated in Figure 4.42 and Figure 4.43, the photo-Fenton process $\left(\mathrm{UV} / \mathrm{Fe}^{3+}\right)$ in the single photoreactor and two photoreactors in series did not show high TOC removal efficiencies (less than 45\%). This is due to the lower quantum yield of the hydroxyl radical production by Reaction (4.94) (0.2 mol.Einstein $\left.{ }^{-1}\right)$ than the quantum yield of Reaction (4.91) (0.5 mol.Einstein ${ }^{-}$ 1) (Simunovic et al., 2011). The highest TOC removal efficiency was correspondent to the photo- 


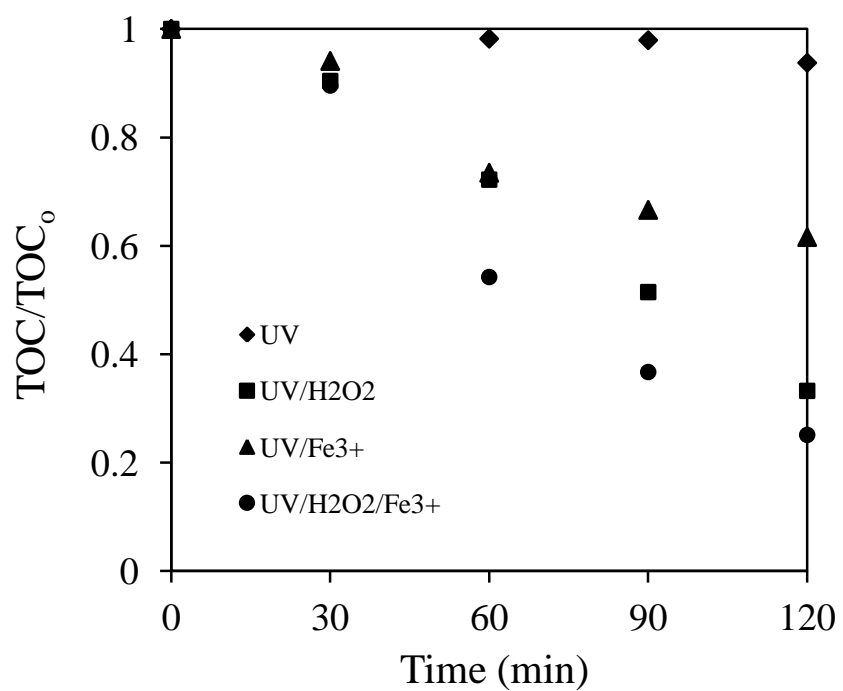

Figure 4.42. TOC removal (norm) in one photoreactor for $\mathrm{UV}, \mathrm{UV} / \mathrm{H}_{2} \mathrm{O}_{2}, \mathrm{UV} / \mathrm{Fe}^{3+}$, and $\mathrm{UV} / \mathrm{H}_{2} \mathrm{O}_{2} / \mathrm{Fe}^{3+}$ processes.

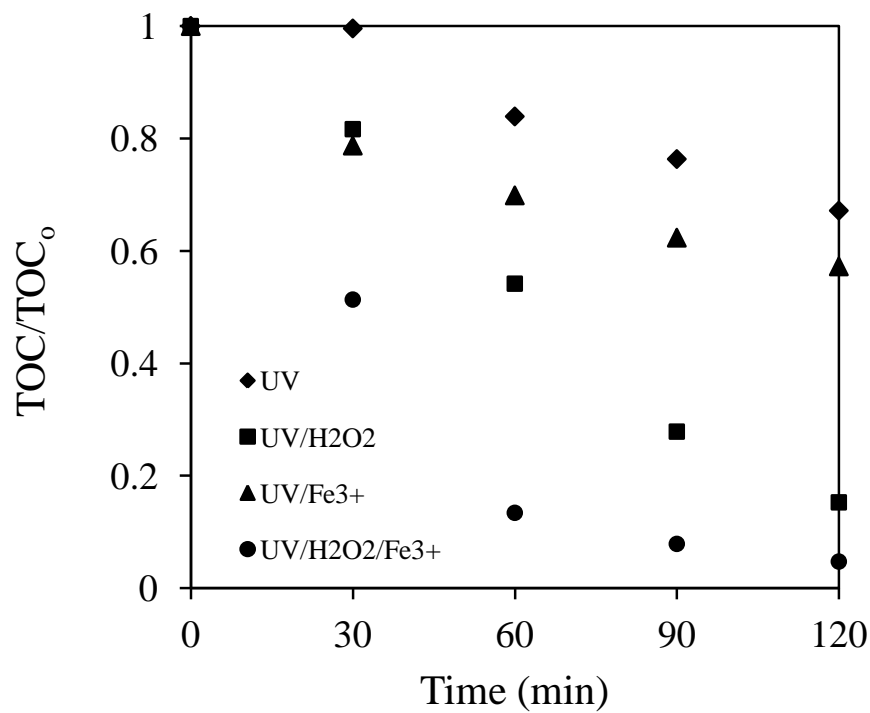

Figure 4.43. TOC removal (norm) in two photoreactors in series for $\mathrm{UV}, \mathrm{UV} / \mathrm{H}_{2} \mathrm{O}_{2}, \mathrm{UV} / \mathrm{Fe}^{3+}$, and $\mathrm{UV} / \mathrm{H}_{2} \mathrm{O}_{2} / \mathrm{Fe}^{3+}$ processes. 
Fenton-like process $\left(\mathrm{UV} / \mathrm{H}_{2} \mathrm{O}_{2} / \mathrm{Fe}^{3+}\right)$ using two photoreactors in series as shown in Figure 4.43. Therefore, the effectiveness of the photo-Fenton-like process was studied using response surface methodology.

\subsubsection{Experimental Design}

A four-factor three-level Box-Behnken experimental design combined with response surface modeling was used to correlate experimentally obtained criteria and experimental conditions given by Box-Behnken experimental design. In the Box-Behnken design, a modified central composite design, the variable combinations are at the center and the midpoints of the edges of the variable space (Ay et al., 2009). Also, compared to other types of experimental design such as full factorial design, the Box-Behnken experimental design needs fewer runs. Therefore, the effects of four independent variables on the response function were investigated. The independent variables were initial concentrations of PAA $\left(\mathrm{X}_{1}\right)$ and $\mathrm{H}_{2} \mathrm{O}_{2}: \mathrm{Fe}^{3+}\left(X_{2}\right), \mathrm{pH}\left(X_{3}\right)$, and recirculation rate $\left(X_{4}\right)$ that were coded as $-1,0$, and +1 as illustrated in Table 4.13 . The total number of experiments in this study was 27 based on three levels and a three factor experimental design, with three replicates at the center of the design to estimate a pure error sum of squares. The percent TOC removal rate and the "pseudo"-second order rate constant were considered as the dependent factors (process responses). The independent variables and their critical experimental levels in Table 4.13 were chosen based on preliminary experimental results and the literature values (Rojas et al., 2010; Silva et al., 2010; Sun and Lemley, 2011). The statistical softwares, STATISTICA (trial version 10.0) and Design-Expert (trial version 8.0), were used for the regression analysis and the parameter estimation of the response functions. The statistical significance of the model equations was completely analyzed using analysis of variance 
(ANOVA) at $95 \%$ confidence interval. Three-dimensional response surface plots and twodimensional contour plots were developed while holding a variable constant in the quadratic model.

Table 4.13. Independent variables and their coded levels based on Box-Behnken design for photo-Fenton-like process using two photoreactors in series.

\begin{tabular}{llllll}
\hline \multirow{2}{*}{ Independent Variable } & Unit & Symbol & \multicolumn{3}{c}{ Coded Levels } \\
\cline { 3 - 5 } & & & -1 & 0 & 1 \\
\hline & & & 10 & 30 & 50 \\
{$[\mathrm{PAA}]_{\mathrm{o}}$} & $\mathrm{mg} \cdot \mathrm{L}^{-1}$ & $X_{1}$ & $400: 4$ & $900: 6$ & $1600: 8$ \\
{$\left[\mathrm{H}_{2} \mathrm{O}_{2}: \mathrm{Fe}^{3+}\right]_{\mathrm{o}}$} & $\mathrm{mg} \cdot \mathrm{L}^{-1}: \mathrm{mg} \cdot \mathrm{L}^{-1}$ & $X_{2}$ & 3 & 5 & 7 \\
$\mathrm{pH}$ & - & $X_{3}$ & 1 & 6 & 10 \\
Recirculation rate & $\mathrm{L} \cdot \mathrm{min}^{-1}$ & $X_{4}$ & & & \\
\hline
\end{tabular}

The experimental and predicted values were compared to validate the derived models. The optimal operating conditions to maximize the TOC removal rate were also determined using a numerical technique built in the software Design-Expert 8.0. The "pseudo"-second order rate constant was calculated at the determined optimal conditions. Also, another experimental run was carried out to verify the obtained optimal conditions by the derived models for both response functions.

\subsubsection{RSM Model Development}

The efficiency of the photo-Fenton-like process $\left(\mathrm{UV} / \mathrm{H}_{2} \mathrm{O}_{2} / \mathrm{Fe}^{3+}\right)$ for the treatment of aqueous PAA in a batch recirculation system using two photoreactors in series was investigated using the Box-Behnken experimental design including four factors at three levels. The process parameters taken into account were initial concentrations of PAA and $\mathrm{H}_{2} \mathrm{O}_{2}: \mathrm{Fe}^{3+}, \mathrm{pH}$, and the recirculation 
rate. The effectiveness of the process was evaluated through the TOC removal rate for the model wastewater. Also, the performance of RSM on the "pseudo"-second order rate constant was tested in order to indirectly introduce another parameter (treatment time) in the experimental design (Kusic et al., 2010). In order to compare the reaction rates, the reaction order has to be determined and then the rate constants can be easily calculated and compared through the linear regression if all of them could be fitted to the same reaction order (Dopar et al., 2010). Therefore, several functional dependences of the TOC concentration on time representing different integral order of Reactions (zero, first, and second) were examined. It was determined that the reaction rates of the TOC removal for all 27 runs were fitted well to the "pseudo"-second order kinetics $\left(1 / \mathrm{TOC}_{\mathrm{t}}-1 / \mathrm{TOC}_{\mathrm{o}}=k_{\mathrm{obs}} \times t\right)$ as shown in Figure 4.44. The range of linear regression coefficients $\left(R^{2}\right)$ was from 0.91 to 0.99 . The four-factor Box-Behnken experimental design and the observed and predicted values for the percent TOC removal rates and the reaction rate constants by the derived quadratic models are shown in Table 4.14. As mentioned earlier, the RSM was used to estimate the parameters indicating an empirical relationship between input variables and the responses. Therefore, the quadratic model equation for predicting the percent TOC removal rate $\left(Y_{1}\right)$ and the pseudo"-second order rate constant $\left(Y_{2}\right)$ are presented in the following second order polynomial equations:

$$
\begin{aligned}
& Y_{1}=89.63-3.66 X_{1}+2.16 X_{2}-6.26 X_{3}+0.21 X_{4}+1.26 X_{1} X_{2}-1.08 X_{1} X_{3}+0.26 X_{1} X_{4}+ \\
& 0.38 X_{2} X_{3}+0.33 X_{2} X_{4}-0.075 X_{3} X_{4}-0.91 X_{1}^{2}-0.46 X_{2}{ }^{2}-3.10 X_{3}{ }^{2}+0.29 X_{4}{ }^{2} \\
& Y_{2}=19-23.42 X_{1}+4.25 X_{2}-10.17 X_{3}+1.83 X_{4}+0.5 X_{1} X_{2}+8 X_{1} X_{3}-1.25 X_{1} X_{4}+ \\
& 0.75 X_{2} X_{3}-1.5 X_{2} X_{4}+2.25 X_{3} X_{4}+14.58 X_{1}{ }^{2}+1.58 X_{2}{ }^{2}+1.21 X_{3}{ }^{2}+4.21 X_{4}{ }^{2}
\end{aligned}
$$

where $X_{1}, X_{2}, X_{3}$, and $X_{4}$ are coded terms for four independent variables including the initial concentrations of PAA and $\mathrm{H}_{2} \mathrm{O}_{2}: \mathrm{Fe}^{3+}, \mathrm{pH}$, and the recirculation rate, respectively. The negative 
coefficients for the model components indicated the unfavorable effects on the percent TOC removal and "pseudo"-second order rate constant, while, the positive coefficients indicated favorable effects on the percent TOC removal and "pseudo"-second order rate constant. The significance of each model parameter is determined by statistical analysis.

\subsubsection{Statistical Analysis}

ANOVA was applied as the most important test to evaluate the significance of the developed quadratic model for the prediction of the percent TOC removal and the pseudo"-second order rate constant. The results of the ANOVA are presented in Table 4.15 and Table 4.16 for the percent TOC removal and the "pseudo"-second order rate constant, respectively. The significance of each coefficient in Equations (4.96) and (4.97) was determined by the Fisher's $F$ test with values of probability greater than $F$. The acceptance or the rejection of the model terms were based on the $p$-value with a 95\% confidence level. As shown in Table 4.15 and Table 4.16, small probability values $(p<0.0001)$ indicate that the models were highly significant which confirm the accuracy of the derived models to predict the response functions. Also, high values of regression coefficient $\left(R^{2}\right)$ as well as adjusted regression coefficient $\left(R_{\text {adj }}^{2}\right)$ ensure an acceptable modification of the quadratic model to the experimental data. In fact, $R_{\text {adj }}^{2}$ modifies $R^{2}$ for the sample size and the number of terms in the model. The values of $R^{2}$ and $R_{\text {adj }}^{2}$ were found to be 0.9363 and 0.8620 for the percent TOC removal and 0.9576 and 0.9081 for the "pseudo"second order rate constants, respectively. The closer $R^{2}$ and $R_{\text {adj }}^{2}$ are to 1 , the better models predict the percent TOC removal and rate constants. The high values of regression and adjusted regression coefficients indicate that the derived quadratic models can adequately describe the 
system behavior within the selected range of operating parameters. Also, the adequate precisions, signal to noise ratio, greater than 4 (12.80 for TOC removal and 15.69 for the "pseudo"-second order rate constant) show that the models could be used to navigate the design space. The high correlations between observed and predicted data are illustrated in Figure 4.45a and Figure 4.46a. As a result of low discrepancies, the points are very close to the diagonal line. The normality of the data could be checked through the normal probability plot of the residuals as shown in Figure 4.45b and Figure 4.46b. Since the points on the plot follow a straight line, it can be concluded that the residuals are normally distributed. Also, as the S-shaped curve is not formed in the Figure $4.45 \mathrm{~b}$ and Figure $4.46 \mathrm{~b}$, the response transformations are not needed (Tanyildizi, 2011). The assumption of constant variance was tested by plotting internally studentized residual versus predicted values as illustrated in Figure $4.45 \mathrm{c}$ and Figure 4.46c. The studentized residuals are found by dividing the residuals by their standard deviations. As is evident from these figures, the points are scattered randomly between the outlier detection limits -3 and +3 . Therefore, it can be concluded that the predictions of experimental data by the derived quadartic models for both the percent TOC removal rate and the "pseudo"-second order rate constants are quite satisfactory.

\subsubsection{Effect of Model Parameters and their Interactions}

As the significance of the models was confirmed (Table 4.15 and 4.16 ) and the predictivity of the experimental data by the derived models found to be accurate (Figure 4.45 and Figure 4.46), the next step is to evaluate the significance of each model parameter. Hence, the most influential process parameters on the percent TOC removal rate and the "pseudo"-second order rate constants can be determined. 

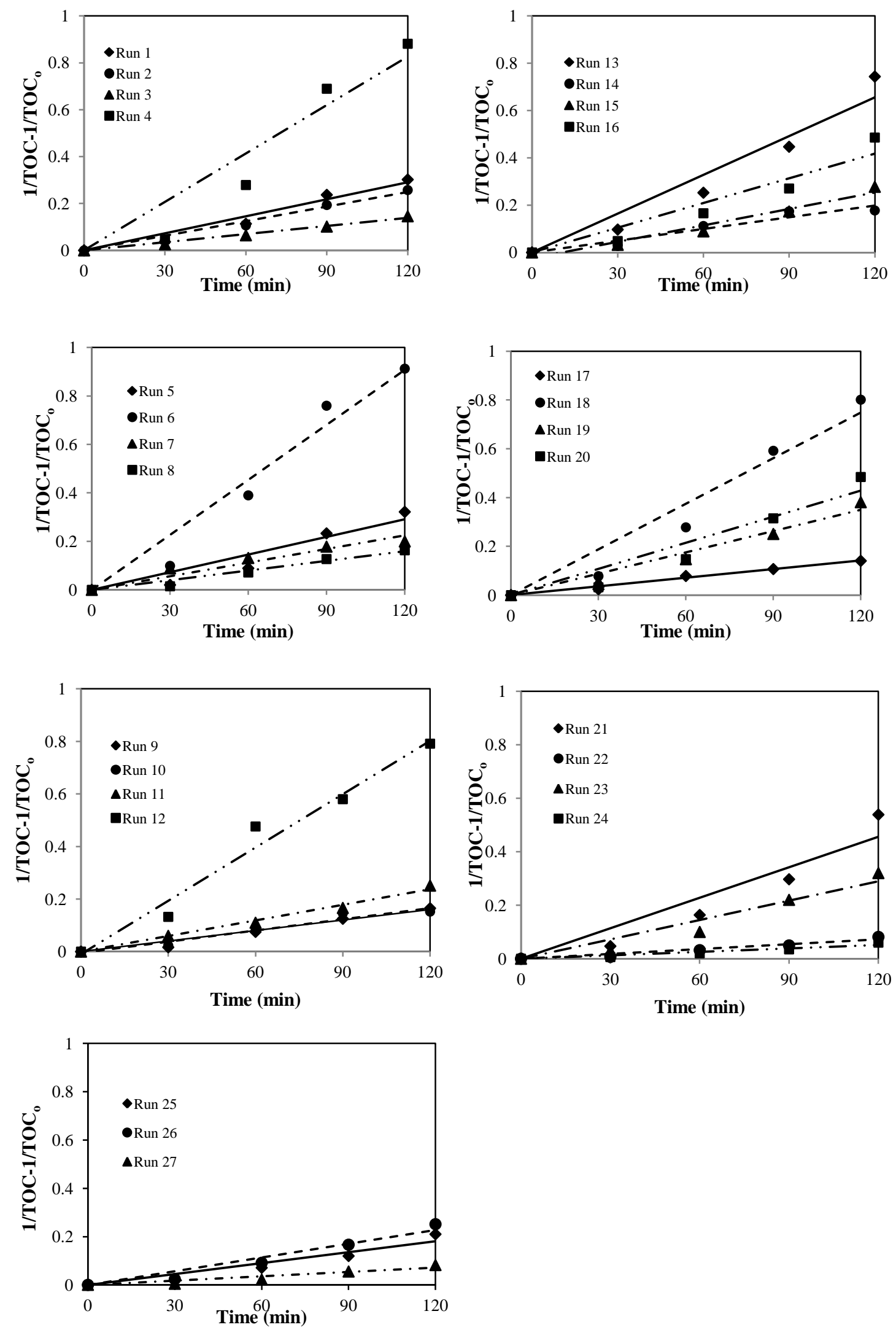

Figure 4.44. "Pseudo"-second order kinetic fit for experimentally achieved TOC in 27 runs. 
Table 4.14. Four-factor Box-Behnken design and the observed and predicted responses.

\begin{tabular}{|c|c|c|c|c|c|c|c|c|}
\hline \multirow[t]{2}{*}{ Run } & \multicolumn{4}{|c|}{ Independent Coded Variables } & \multicolumn{2}{|c|}{ TOC Removal (\%) } & \multicolumn{2}{|c|}{$k_{\mathrm{obs}} \times 10^{-4}\left(\mathrm{M}^{-1} \cdot \mathrm{s}^{-1}\right)$} \\
\hline & $X_{1}$ & $X_{2}$ & $X_{3}$ & $X_{4}$ & Observed & Predicted & Observed & Predicted \\
\hline 1 & 0 & 1 & 0 & -1 & 89.98 & 91.08 & 24 & 29 \\
\hline 2 & 0 & -1 & 0 & 1 & 87.55 & 87.18 & 21 & 24 \\
\hline 3 & 0 & 0 & 1 & 1 & 80.98 & 80.84 & 14 & 18 \\
\hline 4 & -1 & 1 & 0 & 0 & 95.13 & 92.82 & 69 & 62 \\
\hline 5 & 0 & 1 & 0 & 1 & 90.30 & 92.16 & 24 & 29 \\
\hline 6 & -1 & 0 & -1 & 0 & 95.65 & 94.46 & 74 & 76 \\
\hline 7 & 1 & 1 & 0 & 0 & 88.21 & 88.02 & 22 & 16 \\
\hline 8 & 1 & 0 & 0 & -1 & 87.53 & 85.40 & 13 & 14 \\
\hline 9 & 1 & 0 & 0 & 1 & 87.86 & 85.30 & 13 & 15 \\
\hline 10 & 0 & 0 & 1 & -1 & 80.17 & 80.27 & 14 & 10 \\
\hline 11 & 0 & 0 & 0 & 0 & 89.26 & 89.63 & 19 & 19 \\
\hline 12 & -1 & 0 & 0 & 1 & 91.20 & 93.14 & 67 & 64 \\
\hline 13 & -1 & -1 & 0 & 0 & 89.86 & 91.02 & 55 & 55 \\
\hline 14 & 0 & 1 & 1 & 0 & 83.34 & 82.35 & 18 & 17 \\
\hline 15 & 0 & 0 & 0 & 0 & 88.97 & 89.63 & 19 & 19 \\
\hline 16 & 0 & 0 & -1 & -1 & 91.94 & 92.94 & 35 & 35 \\
\hline 17 & 0 & -1 & 1 & 0 & 79.49 & 77.27 & 12 & 7 \\
\hline 18 & -1 & 0 & 0 & -1 & 92.68 & 92.20 & 62 & 58 \\
\hline 19 & -1 & 0 & 1 & 0 & 82.89 & 84.10 & 29 & 40 \\
\hline 20 & 0 & 0 & -1 & 1 & 92.35 & 93.21 & 36 & 34 \\
\hline 21 & 0 & 1 & -1 & 0 & 93.66 & 94.10 & 32 & 35 \\
\hline 22 & 1 & -1 & 0 & 0 & 77.91 & 81.18 & 6 & 7 \\
\hline 23 & 0 & -1 & -1 & 0 & 91.34 & 90.55 & 29 & 28 \\
\hline 24 & 1 & 0 & 1 & 0 & 72.61 & 74.62 & 4 & 9 \\
\hline 25 & 0 & -1 & 0 & -1 & 88.44 & 87.42 & 15 & 17 \\
\hline 26 & 0 & 0 & 0 & 0 & 90.70 & 89.26 & 19 & 19 \\
\hline 27 & 1 & 0 & -1 & 0 & 89.71 & 89.30 & 17 & 14 \\
\hline
\end{tabular}

This evaluation is based on the $F$ and $p$-values for each of the linear, quadratic, and interaction model paramaters as listed in the Table 4.15 and 4.16. Generally, the Fischer's $F$-value is used to determine the significance of regression coefficients of the variables while $p$-value determeines the significance of the variable that may indicate a pattern of interaction among variables. The larger the value of $F$ and the smaller the value of $p$ are, the more siginificant the corresponding coefficient is (Bayraktar, 2001). As shown in Table 4.15 and 4.16, the $p$-value less than 0.05 indicates the model parameters are significant. 
Table 4.15. ANOVA results for prediction of percent TOC removal in photo-Fenton-like process using two photoreactors in series.

\begin{tabular}{|c|c|c|c|c|c|}
\hline \multirow{2}{*}{$\begin{array}{l}\text { Factors } \\
\text { (coded) }\end{array}$} & \multicolumn{5}{|c|}{ Statistics } \\
\hline & $\mathbf{S S}^{1}$ & $d f^{2}$ & $\mathrm{MS}^{3}=\mathrm{SS} / \mathrm{df}$ & $F$-value & $\begin{array}{l}p \text {-value }{ }^{4} \\
\text { Prob }>F\end{array}$ \\
\hline Model & 761.57 & 14 & 54.40 & 12.60 & $<0.0001$ \\
\hline$X_{1}$ & 160.60 & 1 & 160.60 & 37.20 & $<0.0001$ \\
\hline$X_{2}$ & 56.03 & 1 & 56.03 & 12.98 & 0.0036 \\
\hline$X_{3}$ & 469.63 & 1 & 469.63 & 108.77 & $<0.0001$ \\
\hline$X_{4}$ & 0.55 & 1 & 0.55 & 0.13 & 0.7283 \\
\hline$X_{1} X_{2}$ & 6.33 & 1 & 6.33 & 1.47 & 0.2494 \\
\hline$X_{1} X_{3}$ & 4.71 & 1 & 4.71 & 1.09 & 0.3169 \\
\hline$X_{1} X_{4}$ & 0.28 & 1 & 0.28 & 0.064 & 0.8048 \\
\hline$X_{2} X_{3}$ & 0.59 & 1 & 0.59 & 0.14 & 0.7192 \\
\hline$X_{2} X_{4}$ & 0.43 & 1 & 0.43 & 0.099 & 0.7580 \\
\hline$X_{3} X_{4}$ & 0.023 & 1 & 0.023 & $5.21 \mathrm{E}-003$ & 0.9436 \\
\hline$X_{1}^{2}$ & 4.39 & 1 & 4.39 & 1.02 & 0.3331 \\
\hline$X_{2}^{2}$ & 1.15 & 1 & 1.15 & 0.27 & 0.6156 \\
\hline$X_{3}^{2}$ & 51.21 & 1 & 51.21 & 11.86 & 0.0049 \\
\hline$X_{4}^{2}$ & 0.43 & 1 & 0.43 & 0.10 & 0.7569 \\
\hline Residual & 51.81 & 12 & \multicolumn{2}{|c|}{4.32} & \\
\hline Pure Error & 0.66 & 2 & \multicolumn{2}{|c|}{0.33} & \\
\hline
\end{tabular}

As presented in Tables 4.15 and 4.16, the initial concentrations of PAA and $\mathrm{H}_{2} \mathrm{O}_{2}: \mathrm{Fe}^{3+}$, as well as $\mathrm{pH}$ have significent linear effects on the percent TOC removal rate and "pseudo"-second order rate constants. The effect of the initial concentration of PAA on the percent TOC removal rate and "pseudo"-second order rate constants is mainly due to the absorption of UV radiation by PAA molecules. An increase in the initial PAA concentration results in inducing an inner filter effect, i.e., the UV light penetration becomes less due to less permeability of the solution (Xu et al., 2009). This leads to a decrease in the UV radiation absorption by $\mathrm{H}_{2} \mathrm{O}_{2}$ and $\mathrm{Fe}^{3+}$ and subsequently less hydroxyl radical formation which is the main cause of the PAA degradation. Also, the high amount of PAA molecules reduces the chance of being in contact with the hydroxyl radicals and consequently fewer molecules are attacked by the hydroxyl radicals. According to Reactions (4.93) and (4.94), higher concentration of hydrogen peroxide and ferric 
Table 4.16. ANOVA results for prediction of "pseudo"-second order rate constant in photo-Fentonlike process using two photoreactors in series.

\begin{tabular}{llllll}
\hline $\begin{array}{l}\text { Factors } \\
\text { (coded) }\end{array}$ & \multicolumn{5}{c}{ Statistics } \\
\cline { 2 - 6 } & SS & df & MS=SS/df & $\boldsymbol{F}$-value & $\begin{array}{l}\boldsymbol{p} \text {-value } \\
\text { Prob }>\boldsymbol{F}\end{array}$ \\
\hline Model & 9625.94 & 14 & 687.57 & 19.34 & $<0.0001$ \\
$X_{1}$ & 6580.08 & 1 & 6580.08 & 185.10 & $<0.0001$ \\
$X_{2}$ & 216.75 & 1 & 216.75 & 6.10 & 0.0295 \\
$X_{3}$ & 1240.33 & 1 & 1240.33 & 34.89 & $<0.0001$ \\
$X_{4}$ & 40.33 & 1 & 40.33 & 1.13 & 0.3078 \\
$X_{1} X_{2}$ & 1.00 & 1 & 1.00 & 0.028 & 0.8696 \\
$X_{1} X_{3}$ & 256.00 & 1 & 256.00 & 7.20 & 0.0199 \\
$X_{1} X_{4}$ & 6.25 & 1 & 6.25 & 0.18 & 0.6824 \\
$X_{2} X_{3}$ & 2.25 & 1 & 2.25 & 0.063 & 0.8056 \\
$X_{2} X_{4}$ & 9.00 & 1 & 9.00 & 0.25 & 0.6240 \\
$X_{3} X_{4}$ & 20.25 & 1 & 20.25 & 0.57 & 0.4650 \\
$X_{1}{ }^{2}$ & 1134.26 & 1 & 1134.26 & 31.91 & 0.0001 \\
$X_{2}{ }^{2}$ & 13.37 & 1 & 13.37 & 0.38 & 0.5511 \\
$X_{3}{ }^{2}$ & 7.79 & 1 & 7.79 & 0.22 & 0.6481 \\
$X_{4}{ }^{2}$ & 94.45 & 1 & 94.45 & 2.66 & 0.1290 \\
Residual & 426.58 & 12 & 35.55 & & \\
Pure Error & 0.000 & 2 & 0.000 & & \\
& & & & & \\
\hline
\end{tabular}

iron results in the formation of more ferrous iron and subsequently more hydroxyl radicals which are the major cause of polymer degradation. In more acidic $\mathrm{pH}$, higher level of dissolved iron will be maintained in aqueous solution. Also, the oxidation potential of hydroxyl radicals is higher at more acidic conditions (Sun and Lemley, 2011). The recirculation rate did not show any significant effect on the response functions showing that this factor can be ignored in comparison to other influential factors. In liquid phase reactions, the recirculation rate or the rate of mixing is usually considered as an important factor. A higher rate of mixing increases the rate of reaction by bringing the reactants together. The insignificant effect of the rate of mixing in the selected range indicates that the small photoreactor volume provides good mixing condition in all studied levels. The interaction model parameters $\left(X_{1} X_{2}, X_{1} X_{3}, X_{1} X_{4}, X_{2} X_{3}, X_{2} X_{4}, X_{3} X_{4}\right)$ as 
shown in Table 4.15 did not show any significant effect on the percent TOC removal while the interaction effect of the initial concentration of polymer and $\mathrm{pH}\left(X_{1} X_{3}\right)$ shows a significant effect on the "pseudo"-second order rate constant. The significant effect of $X_{1} X_{3}$ indicates that the interaction effect of the initial concentration of the polymer and $\mathrm{pH}$ is beneficial to the TOC

removal rate or the treatment time. The quadratic model parameters $X_{3}{ }^{2}$ (the quadratic effect of $\mathrm{pH}$ ) and $X_{1}{ }^{2}$ (the quadratic effect of the initial concentration of the polymer) show significant effects on the percent TOC removal and the "pseudo"-second order rate constant. This finding shows that the percent TOC removal rate is more pronounced by the $\mathrm{pH}$ and the "pseudo"second order rate constant or the treatment time is more affected by the initial concentration of the polymer.

\subsubsection{D Response Surface and 2D Contour Plots}

The three dimensional (3D) response surface and two dimensional (2D) contour plots as the graphical representations of the regression analysis are presented in Figure 4.47 and Figure 4.48 for the percent TOC removal rate and the "pseudo"-second order rate constant, respectively. In such plots, the response function of two factors are presented while all others are at the fixed levels. The 3D response surface plots were formed based on the quadratic model Equations (4.96) and (4.97) and the relationship between the model factors and the response functions were illustrated by corresponding contour plots. The nonlinear nature of all 3D response surfaces in Figure 4.47 and Figure 4.48 show the considerable interactions between each of the independent variables and their corresponding response functions. Figure 4.47a and Figure 4.48a present the influence of the initial concentration of PAA $\left(X_{1}\right)$ and the initial concentration of $\mathrm{H}_{2} \mathrm{O}_{2}: \mathrm{Fe}^{3+}$ 
$\left(X_{2}\right)$ on the percent TOC removal rate and the "pseudo"-second order rate constant in the photoFenton-like process, respectively. As is evident in these figures, higher initial concentrations of PAA have negative effects on the response functions while higher $\mathrm{H}_{2} \mathrm{O}_{2}: \mathrm{Fe}^{3+}$ dosage results in higher TOC removal and higher rate constant. The high negative coefficient of $X_{1}$ in the response functions (Equations (4.96) and (4.97)) also confirms the antagonistic effect of the initial concentrations of PAA on the response functions. Furthermore, as shown in these figures, the effect of the initial concentration of PAA is more influential than the other factors as it is in agreement with the significance of these two process parameters on the basis of p-values (Table 4.15 and 4.16). However, as shown in the ANOVA result tables (Table 4.15 and 4.16), the interaction between the initial concentrations of PAA and the oxidant ratio $\left(\mathrm{H}_{2} \mathrm{O}_{2}: \mathrm{Fe}^{3+}\right)$ does not show any significant effect on the response functions. Figure $4.47 \mathrm{~b}$ and Figure $4.48 \mathrm{~b}$ present the interaction effects between the initial concentration of the polymer and the $\mathrm{pH}$. As is evident in Figure $4.47 \mathrm{~b}$, the higher percent of TOC removal rate at different initial concentration of the polymer is achieved at lower $\mathrm{pH}$ ( 3 to 5 ). As illustrated in Figure $4.48 \mathrm{~b}$, the variation of the initial concentration of the polymer on the "pseudo"-second order rate constant shows more influential at lower pH. $P$-value $<0.05$ in Table 4 also shows the significant effect of the interaction between the initial concentration of PAA and $\mathrm{pH}$ on the "pseudo"-second order rate constant. At low concentration of PAA and low $\mathrm{pH}$, higher rate constant is achieved which could be confirmed by the high negative values of $X_{1}$ and $X_{3}$ coefficients in Equation (4.97). The range of $\mathrm{pH}$ was selected in acidic range as it was proven that in the Fenton-like process a high level of dissolved ferric iron species is achieved at $\mathrm{pH}$ buffered to acidic range (Jung et al., 2009). Also, in the acidic condition, the prolonged reactivity of $\mathrm{H}_{2} \mathrm{O}_{2}$ is higher due to the presence of $\mathrm{H}^{+}$and the lifetime of 
a)

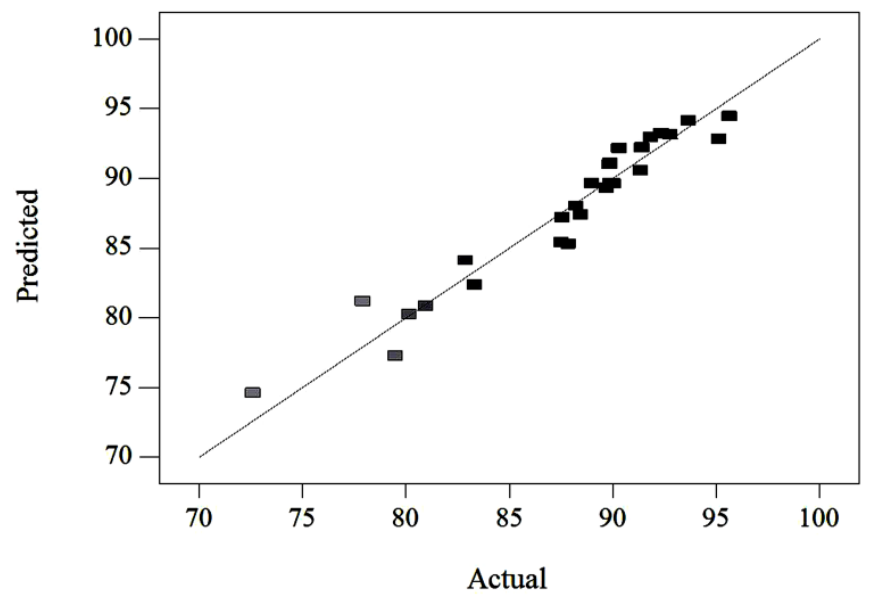

b)

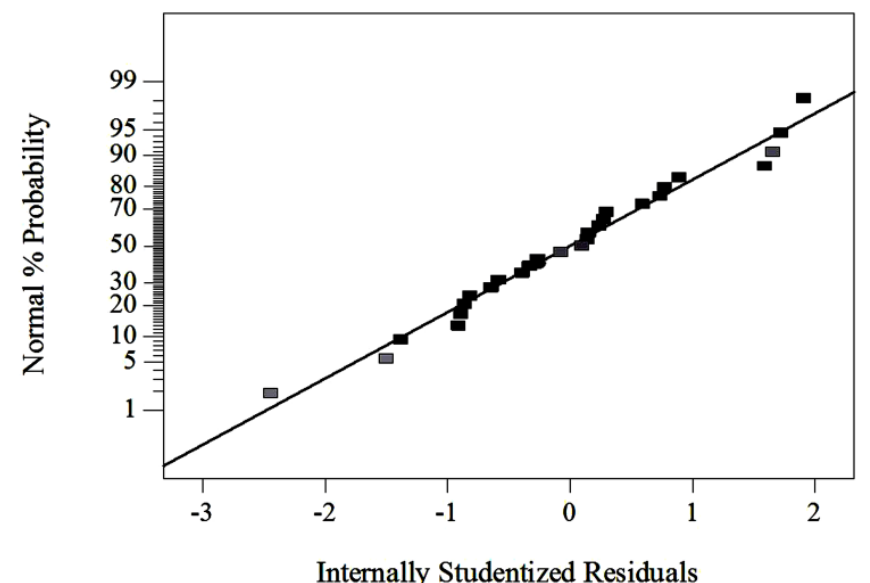

c)

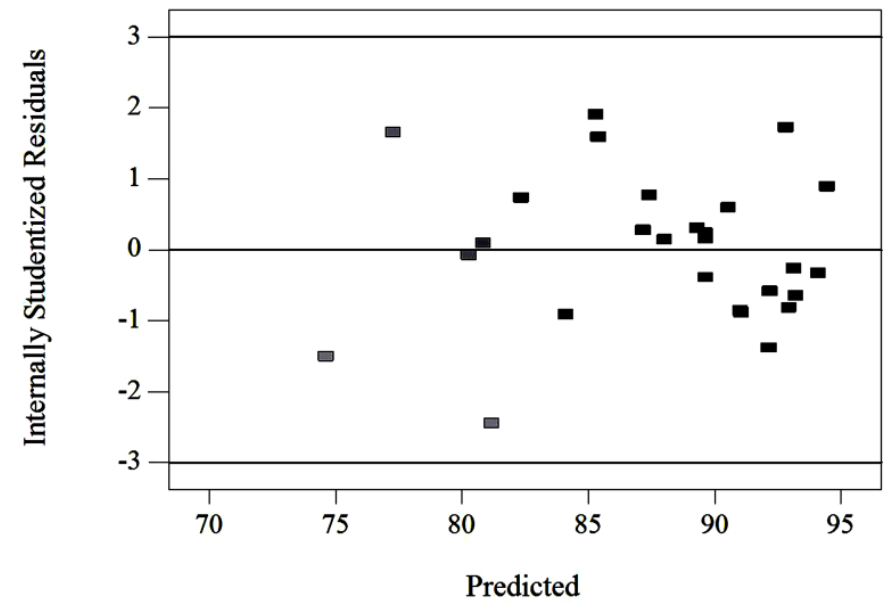

Figure 4.45. Observed vs. predicted plot (a), normal probability plot (b), and internally studentized residuals vs. predicted values plot (c) for the prediction of percent TOC removal in photo-Fenton-like process using two photoreactors in series. 
a)

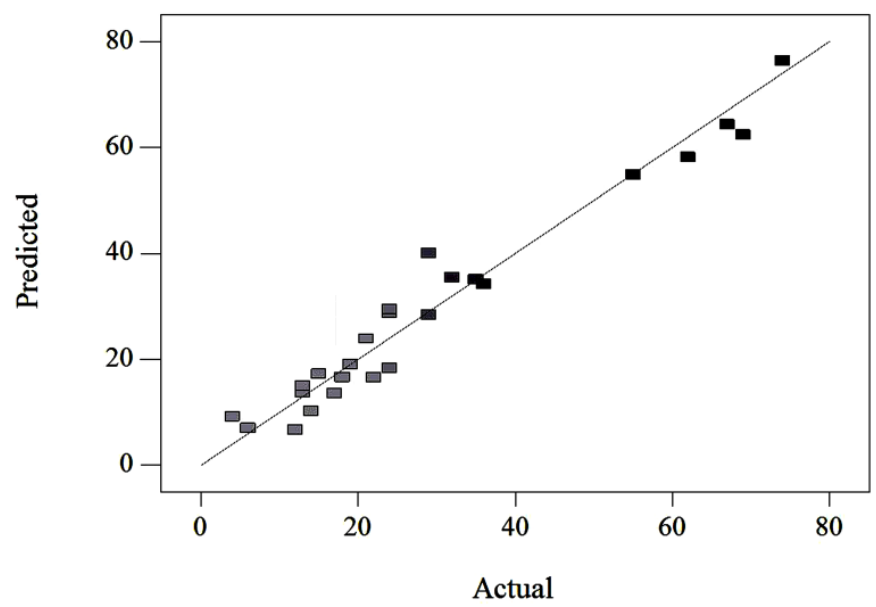

b)

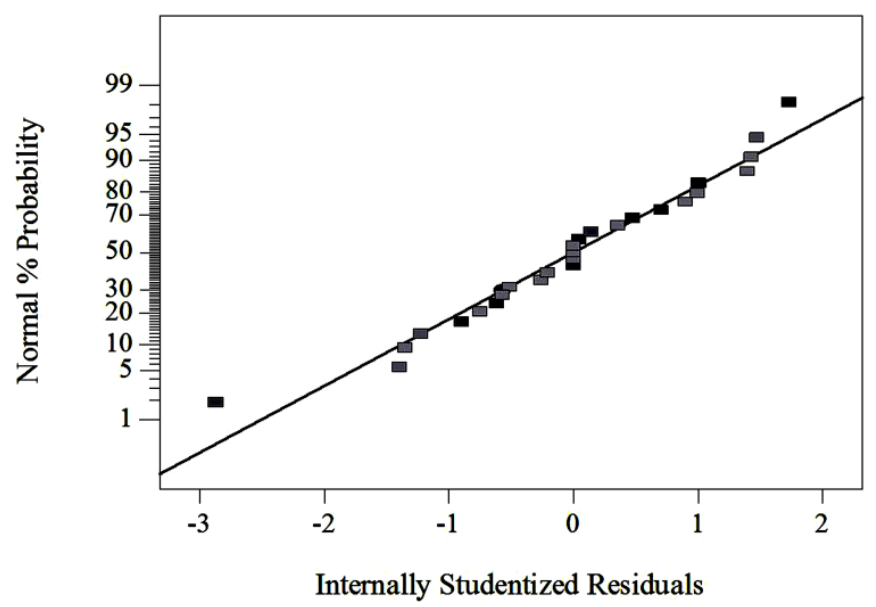

c)

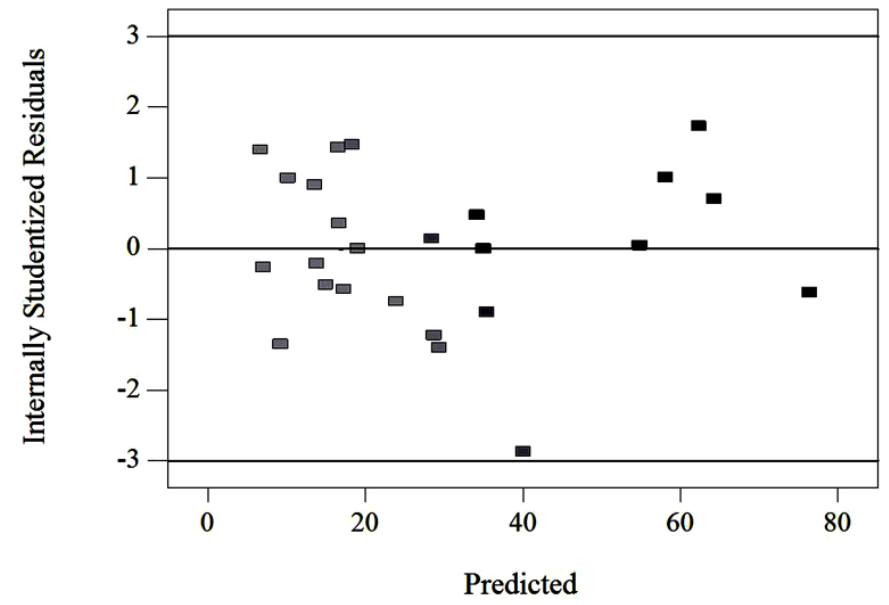

Figure 4.46. Observed vs. predicted plot (a), normal probability plot (b), and internally studentized residuals vs. predicted values plot (c) for the prediction of "pseudo"-second order rate constant in photo-Fenton-like process using two photoreactors in series. 
a)

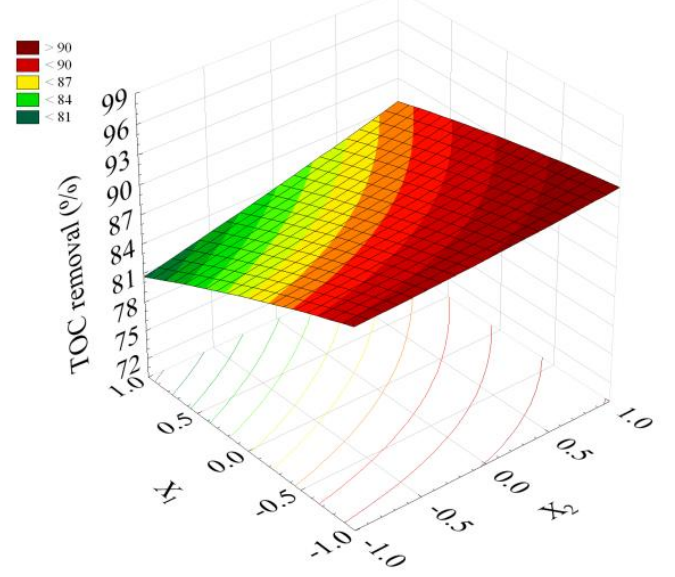

b)

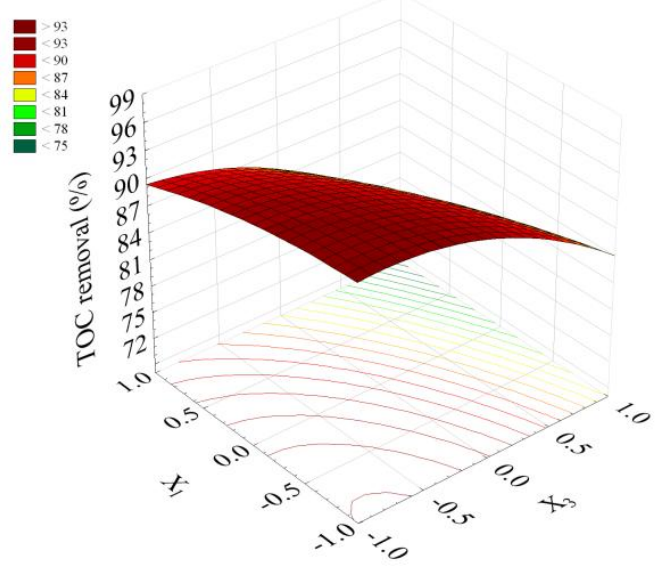

c)

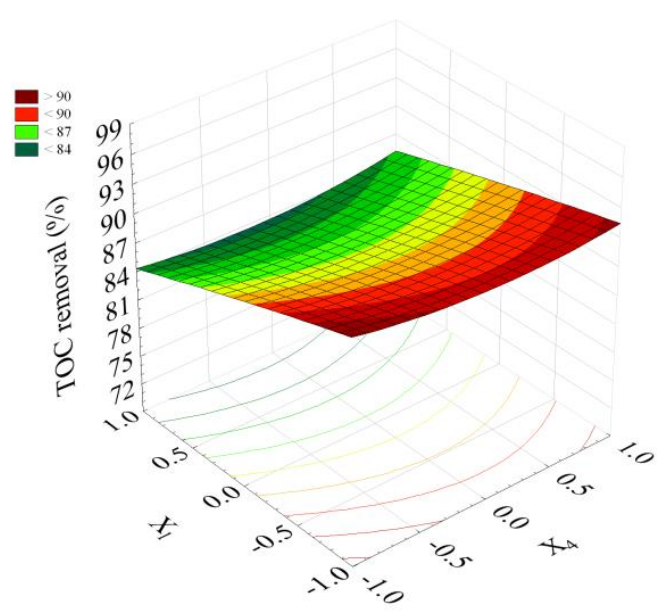

d)

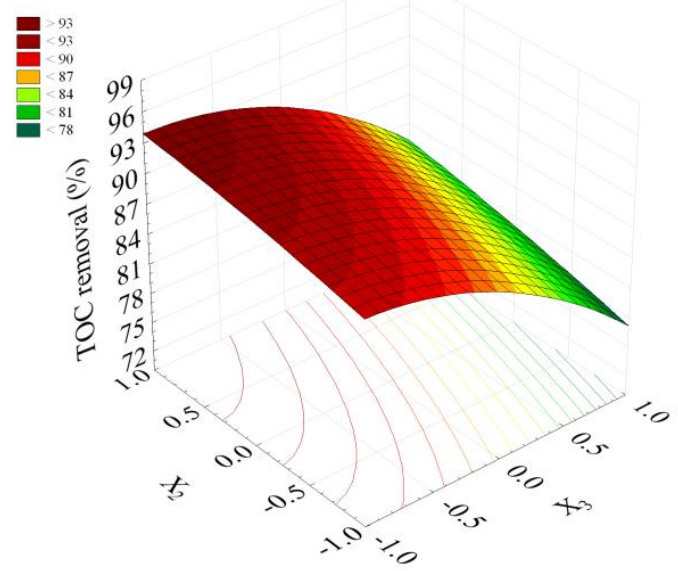

e)

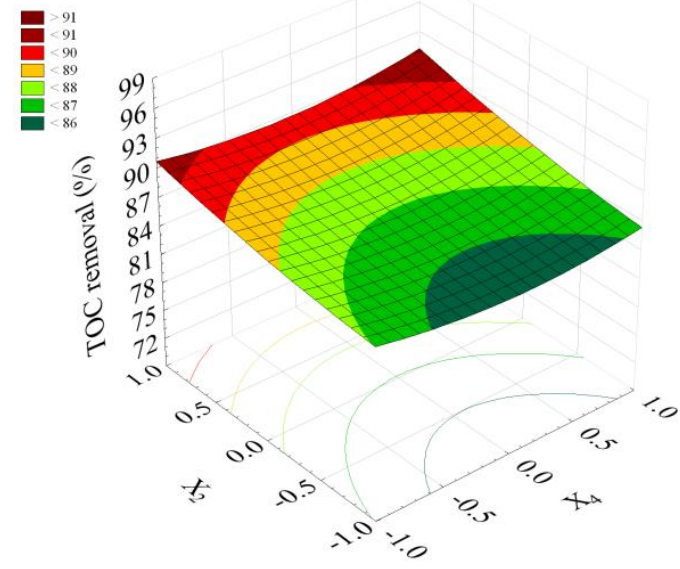

f)

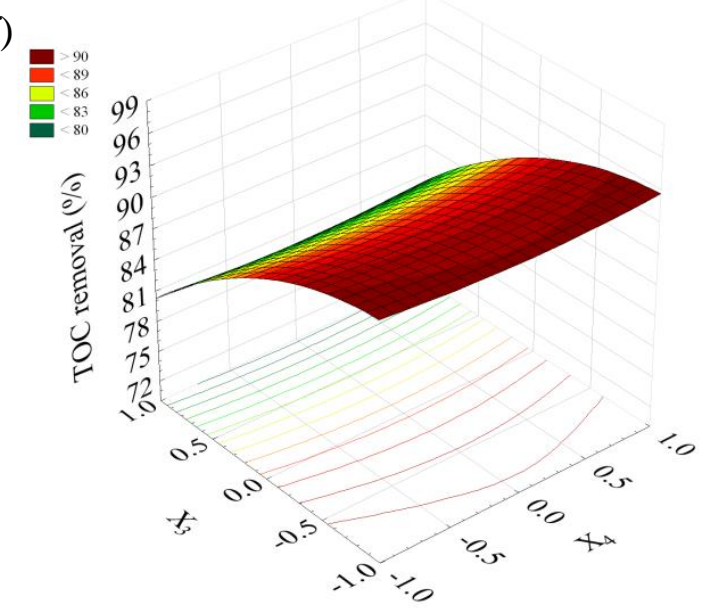

Figure 4.47. $3 \mathrm{D}$ response surface and $2 \mathrm{D}$ contours showing the interaction effects of the factors on the percent TOC removal: (a) $[\mathrm{PAA}]_{\mathrm{o}}\left(X_{1}\right)$ and $\left[\mathrm{H}_{2} \mathrm{O}_{2}: \mathrm{Fe}^{3+}\right]_{\mathrm{o}}\left(X_{2}\right)$, (b) $[\mathrm{PAA}]_{\mathrm{o}}$ and $\mathrm{pH}\left(X_{3}\right)$, (c) $[\mathrm{PAA}]_{\mathrm{o}}$ and recirculation rate $\left(X_{4}\right)$, (d) $\left[\mathrm{H}_{2} \mathrm{O}_{2}: \mathrm{Fe}^{3+}\right]_{\mathrm{o}}$ and $\mathrm{pH}$, (e) $\left[\mathrm{H}_{2} \mathrm{O}_{2}: \mathrm{Fe}^{3+}\right]_{\mathrm{o}}$, (f) $\mathrm{pH}$ and recirculation rate. 
a)

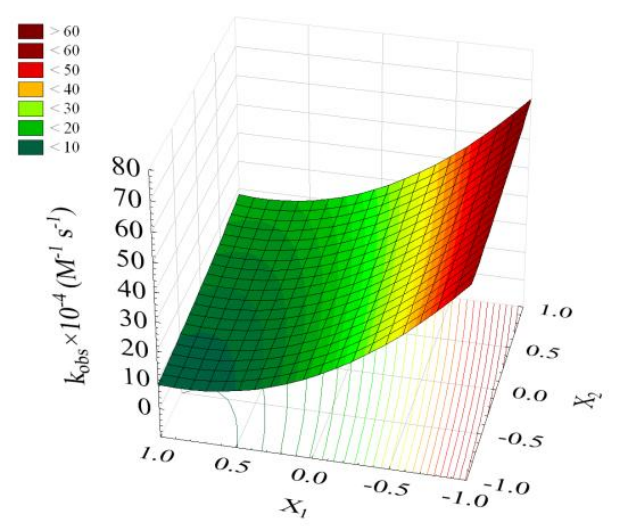

b)

c)
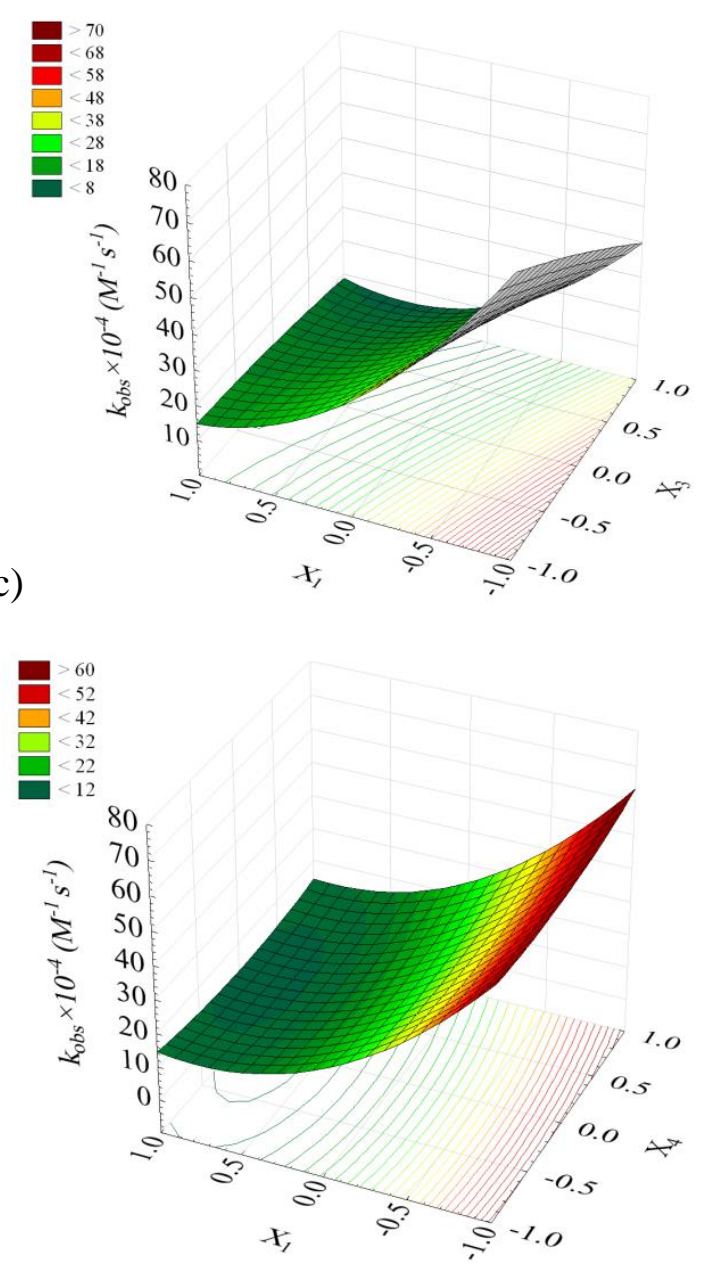

d)

e)

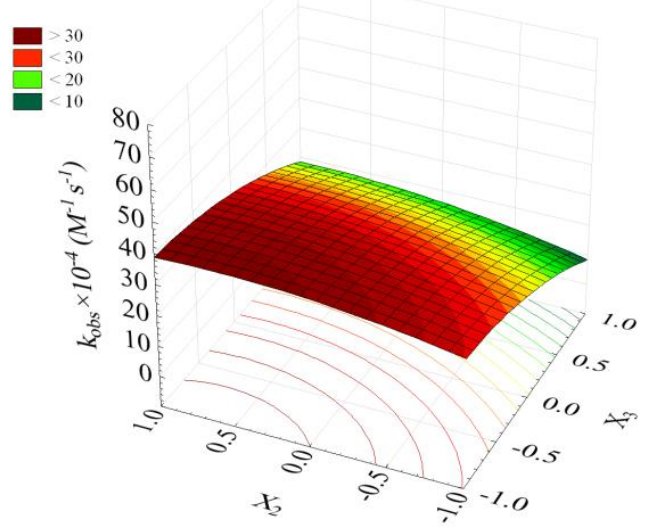

f)
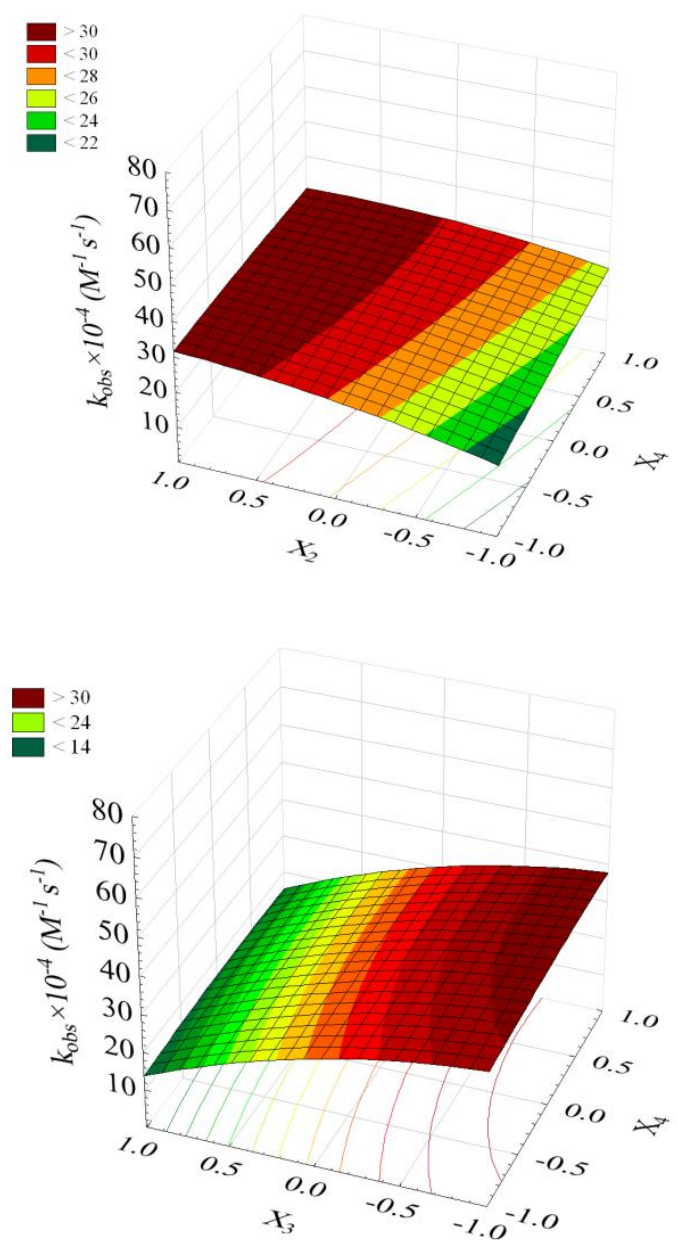

Figure 4.48. $3 \mathrm{D}$ response surface and $2 \mathrm{D}$ contours showing the interaction effects of the factors "pseudo"-second order rate constant: (a) $[\mathrm{PAA}]_{\mathrm{o}}\left(X_{1}\right)$ and $\left[\mathrm{H}_{2} \mathrm{O}_{2}: \mathrm{Fe}^{3+}\right]_{\mathrm{o}}\left(X_{2}\right)$, (b) $[\mathrm{PAA}]_{\mathrm{o}}$ and $\mathrm{pH}$ $\left(X_{3}\right)$, (c) $[\mathrm{PAA}]_{\mathrm{o}}$ and recirculation rate $\left(X_{4}\right)$, (d) $\left[\mathrm{H}_{2} \mathrm{O}_{2}: \mathrm{Fe}^{3+}\right]_{\mathrm{o}}$ and $\mathrm{pH}$, (e) $\left[\mathrm{H}_{2} \mathrm{O}_{2}: \mathrm{Fe}^{3+}\right]_{\mathrm{o}}$ and recirculation rate, $(f) \mathrm{pH}$ and recirculation rate. 
$\mathrm{H}_{2} \mathrm{O}_{2}$ is decreased at buffer $\mathrm{pH}$ as shown in Figure $4.47 \mathrm{~b}$ and Figure 4.48b. As illustrated in Figure $4.47 \mathrm{c}$ and Figure $4.48 \mathrm{c}$ and as confirmed previously in Table 4.15 and 4.16 , the recirculation rate does not show a significant effect on the percent TOC removal and the "pseudo"-second order rate constant. Also, the interaction effects of the initial concentration of the polymer and the recirculation rate on the response functions are insignificant. Figure $4.47 \mathrm{~d}$ illustrates the significant effects of the initial dosage of $\mathrm{H}_{2} \mathrm{O}_{2}: \mathrm{Fe}^{3+}$ and the $\mathrm{pH}$ on the percent TOC removal. As shown in this figure, the $\mathrm{pH}$ changes are more important than the other factor while in Figure 4.48d, both factors show small changes on the "pseudo"-second order rate constant (Figure 4.48d). Figure 4.47e also confirms the significant effect of the oxidants concentration $\left(\mathrm{H}_{2} \mathrm{O}_{2}: \mathrm{Fe}^{3+}\right)$ on the percent TOC removal while Figure 4.48e shows that the changes of the oxidant concentration are less important in the "pseudo"-second order rate constant. Figure $4.47 \mathrm{f}$ also shows the significant effect of the $\mathrm{pH}$ on the TOC removal rate at different recirculation rates while as is evident in Figure 4.48f, the $\mathrm{pH}$ is less influencial factor on the rate constant. Also, as Figure $4.48 \mathrm{f}$ shows, at higher recirculation rates, the $\mathrm{pH}$ decrease has more positive effect on the "pseudo"-second order rate constant.

\subsubsection{Optimization of the Process Parameters}

The derived quadratic model for the percent TOC removal (Equation (4.96)) was used as an objective function to find the optimum operating conditions (the initial PAA concentration, the initial dosage of $\mathrm{H}_{2} \mathrm{O}_{2}: \mathrm{Fe}^{3+}$, $\mathrm{pH}$, and the recirculation rate) that maximize the percent TOC removal. The constraints are the selected ranges for each factor. Therefore, the optimum process parameters were found by using the numerical technique built in the Design Expert Software 8.0.5 based on the predicted model and the factors in the critical range as the constraints. The 
numerical optimization searches the design space using the derived model in the analysis to find factor settings that meet the goal of maximizing the TOC removal rate. The optimum values to achieve the maximum 95.7\% TOC removal rate after 120 min were $10 \mathrm{mg} \mathrm{L}^{-1}$ PAA, $1600 \mathrm{mg} \mathrm{L}^{-1}$ $\mathrm{H}_{2} \mathrm{O}_{2}, 40 \mathrm{mg} \mathrm{L}^{-1} \mathrm{Fe}^{3+}$, $\mathrm{pH} 3.42$, and $10 \mathrm{~L} \mathrm{~min}^{-1}$ recirculation rate, respectively. The "pseudo"second order rate constant at the optimal conditions based on the derived quadratic model (Equation (4.97)) was found to be $82 \mathrm{M}^{-1} \mathrm{~s}^{-1}$. The TOC removal efficiency was tested experimentally at the optimum operating conditions to validate the model prediction. $92.5 \%$ TOC removal and $86 \mathrm{M}^{-1} \cdot \mathrm{s}^{-1}$ "pseudo"-second order rate constant achieved experimentally at the optimal operating conditions confirm the results of the derived models.

\subsubsection{Conclusions}

The photoassisted Fenton-like degradation of PAA as a model water-soluble compound was investigated in this study using two photoreactors in series operating in batch recirculation mode. The initial concentrations of the polymer, $\mathrm{H}_{2} \mathrm{O}_{2}: \mathrm{Fe}^{3+}, \mathrm{pH}$, and the recirculation rate were considered as the process parameters. The percent TOC removal and the "pseudo"-order rate constants were the response functions. The four factor three-level Box-Behnken statistical experiment design applied in this study was found to be an appropriate response surface methodology to determine the effects of process parameters on the response function in the studied range. Two quadratic models predicting the percent TOC removal and the "pseudo"order rate constants were derived. The statistical analysis using ANOVA indicated that the developed quadratic models were highly accurate and predictive. Also, the efficiency of the photo-Fenton like process was strongly affected by the initial concentration of the polymer and

the $\mathrm{pH}$. The oxidant ratio $\left(\mathrm{H}_{2} \mathrm{O}_{2}: \mathrm{Fe}^{3+}\right)$ showed less significant effect on the response functions 
while the recirculation rate had no significant effect on the response functions. The optimal operating conditions to achieve the maximum TOC removal rate (95.7\%) in the selected range was determined to be $10 \mathrm{mg} . \mathrm{L}^{-1}$ PAA, $1600 \mathrm{mg} \mathrm{L}^{-1} \mathrm{H}_{2} \mathrm{O}_{2}, 40 \mathrm{mg} \mathrm{L}^{-1} \mathrm{Fe}^{3+}$, pH 3.42, and $10 \mathrm{~L} \mathrm{~min}^{-}$ ${ }^{1}$ recirculation rate, respectively. The "pseudo"-order rate constant at the optimum conditions for the TOC removal was found to be $82 \mathrm{M}^{-1} \mathrm{~s}^{-1}$. The obtained optimal operating conditions were also validated experimentally. A good agreement between the model prediction and the experimental data confirmed the accuracy of the derived models. 


\subsection{PHOTOREACTOR SCALE-UP FOR DEGRADATION OF \\ POLYVINYL ALCOHOL IN AQUEOUS SOLUTION USING UV/H $\mathrm{H}_{2}$ PROCESS}

\subsubsection{Introduction}

As mentioned in Section 2.5, the traditional and empirical methodology for a photoreactor scaleup, which is carried out by gradual increase of the photoreactor size, is time-consuming and expensive. Therefore, another approach has to be taken into account to avoid the expensive and time-consuming procedure. One of the most robust methods to design a commercial scale photoreactor is by using a precise mathematical modeling supported by accurate laboratory experiments carried out in small photoreactors. By applying this procedure properly, it is possible to proceed from a laboratory-scale photoreactor to a large-scale one avoiding the expensive and time-consuming intermediate steps. In order to achieve this goal, it is always important to design a laboratory photoreactor with the simplest mathematical representation for both mass and radiation balances. In this case, the intrinsic kinetic parameters achieved in the laboratory-scale photoreactor are applied to the commercial-scale photoreactor with different configuration and mode of operation.

In this section, a methodology for a photoreactor scale-up for the photodegradation of polyvinyl alcohol (PVA) using $\mathrm{UV} / \mathrm{H}_{2} \mathrm{O}_{2}$ process is proposed. In this procedure, the intrinsic kinetic parameters are determined using a laboratory-scale batch recirculating photoreactor. The obtained kinetic parameters are used to predict the performance of the pilot-scale continuous flow through photoreactor without any adjustable parameter. In both cases, mass and radiation 
balances are needed. The method is validated in a pilot-scale photoreactor for different geometries using CFD model development.

\subsubsection{Photoreactor Scale-up Methodology}

The photoreactor scale-up methodology in this study is presented in Figure 4.49 which is based on the general methodology to scale-up photoreactors (Alfano and Cassano, 2009). As shown in this figure, the procedure consists of two parts. Part (a) is correspondent to the determination of the intrinsic kinetic parameters in the laboratory-scale photoreactor that are independent of the photoreactor geometry and operating conditions. For this purpose, the kinetic expressions are developed based on the detailed kinetic scheme incorporating the photochemical reactions and all chemical species present in the aqueous solution. A simple kinetic model such as LangmuirHinshelwood only considers the reactants and products concentration while the effect of the oxidizer concentration and radiation flux is not taken into account explicitly. The radiation balance equation is developed based on optical properties of the laboratory-scale photoreactor. The radiation model and kinetic expressions are incorporated in the mass balance of the species involved to achieve the theoretical evolution of each species. In the next step, an optimization algorithm is applied to minimize the error between model predictions and experimental results and consequently estimating the kinetic parameters. The achieved kinetic parameters are independent of the photoreactor geometry and operating conditions and could be applied directly to the kinetic model incorporated in the mass balance of the pilot-scale photoreactor. The second part (b) in Figure 4.49 involves the pilot-scale photoreactor and the application of the kinetic model on that. The 3-dimensional radiation balance and the mass balance are solved in the pilotscale photoreactor. In order to validate the methodology, the experimental results from the pilot- 
scale photoreactor are compared with the simulation results achieved by the CFD model development incorporating the proposed mass and radiation balances.

\subsubsection{Experimental Procedure}

In order to obtain kinetic parameters using the optimization algorithm, experiments were carried out at different $\mathrm{H}_{2} \mathrm{O}_{2}$ dosage $\left(300,500,700,900\right.$, and $\left.1100 \mathrm{mg} \mathrm{L}^{-1}\right)$. The $\mathrm{pH}$ was at natural level of the solution and the initial PVA concentration was $20 \mathrm{mg} \mathrm{L}^{-1}$. The PVA solution was prepared by gradually dissolving PVA in a $300-\mathrm{mL}$ beaker containing distilled water with a magnetic stir bar with the gradual increase of the temperature up to $80^{\circ} \mathrm{C}$. The solution was then diluted to achieve the desired PVA concentration in a 4-L solution and desired amount of $\mathrm{H}_{2} \mathrm{O}_{2}$ was added to the solution at the beginning of each experiment. The UV lamp was then turned on for $30 \mathrm{~min}$ before the beginning of each experiment to stabilize the light intensity and to degrade any possible organic background such as natural organic matter. The solution was fed to the system and the temperature was kept constant at $22^{\circ} \mathrm{C}$ during each experiment by means of a heat exchanger. The samples were taken from the collection tank at time 0 and at time interval of 30 min during total reaction time of $150 \mathrm{~min}$. The TOC concentration of the samples was measured by the TOC analyzer (Apollo 9000, Teledyne Tekmar, USA). The UV/Visible spectrophotometer (Ultrospec 1100 pro) was used to determine the extinction coefficient of PVA.

\subsubsection{Modeling of the Laboratory-Scale Photoreactor}

As mentioned earlier, in the first part of the scaling-up methodology, a laboratory-scale photoreactor is used in order to estimate the intrinsic kinetic parameters. To achieve this goal, the 


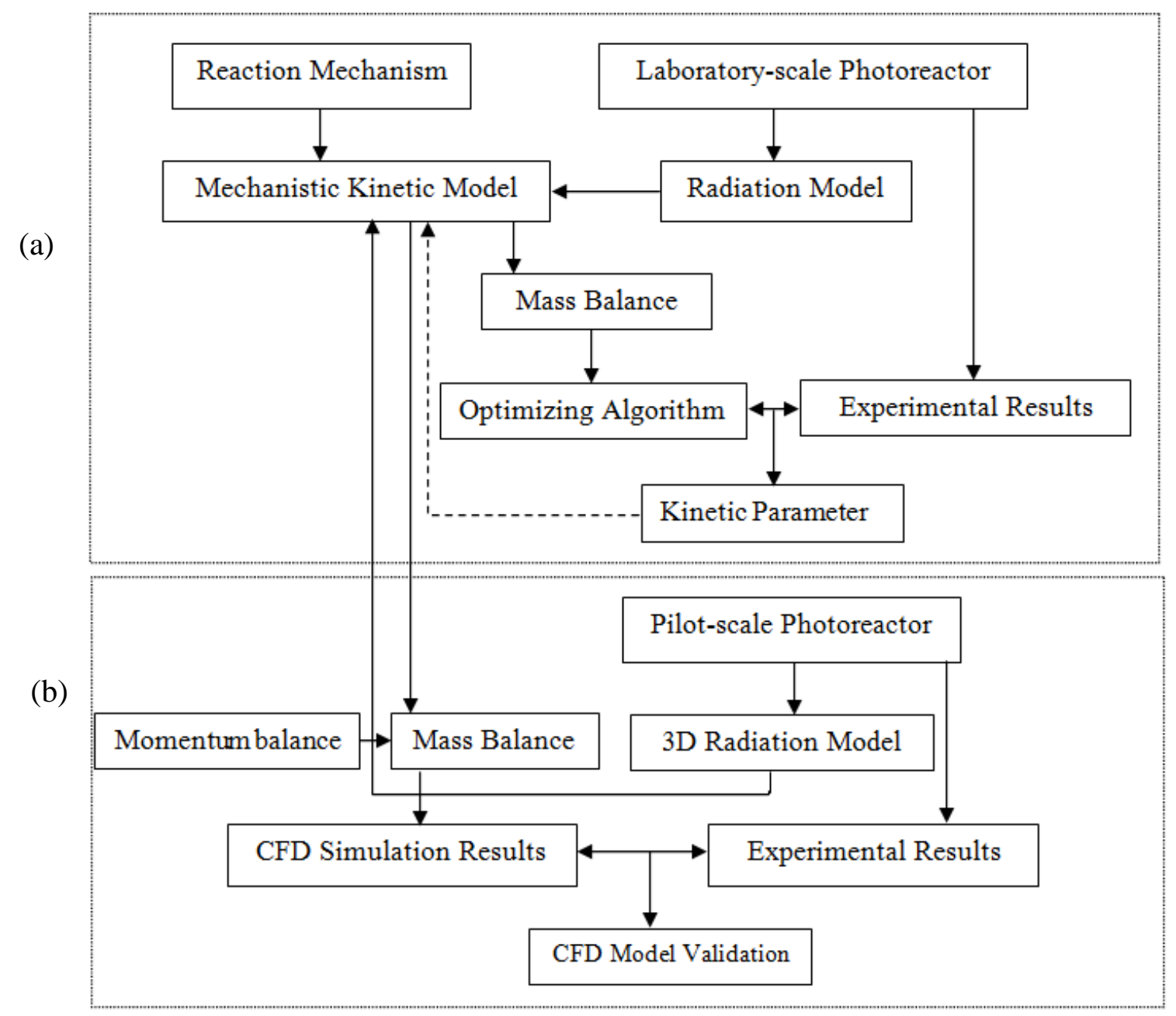

Figure 4.49. Photoreactor Scale-up methodology. 
laboratory-scale batch recirculating system is used as shown in Figure 3.1. The photoreactor configuration enables a simple mathematical representation for both mass and radiation balances.

\subsubsection{Kinetic modeling}

The kinetic model proposed for the photodegradation of PVA by $\mathrm{UV} / \mathrm{H}_{2} \mathrm{O}_{2}$ process is based on the reaction mechanisms in the Table 4.1. A mechanistic kinetic model was also developed based on the reaction sequences in the Table 4.1 as presented in Section 4.1 (Ghafoori et al., 2012a). Therefore, the kinetic expressions for the photodegradation of PVA by $\mathrm{UV} / \mathrm{H}_{2} \mathrm{O}_{2}$ process are as follows.

$$
\begin{aligned}
& R_{\mathrm{H}_{2} \mathrm{O}_{2}}= \\
& \left(-R_{U V, \mathrm{H}_{2} \mathrm{O}_{2}}-k_{2}\left[^{\bullet} \mathrm{OH}\right]\left[\mathrm{H}_{2} \mathrm{O}_{2}\right]-k_{3}\left[^{\bullet} \mathrm{OH}\right]\left[\mathrm{HO}_{2}^{-}\right]-k_{4}\left[\mathrm{HO}_{2}^{\bullet}\right]\left[\mathrm{H}_{2} \mathrm{O}_{2}\right]-k_{5}\left[\mathrm{O}_{2}^{-}\right]\left[\mathrm{H}_{2} \mathrm{O}_{2}\right]+\right. \\
& \left.k_{8}\left[{ }^{\bullet} \mathrm{OH}\right]^{2}+k_{9}\left[H \mathrm{O}_{2}^{\bullet}\right]^{2}+k_{11}\left[H \mathrm{O}_{2}^{\bullet}\right]\left[\mathrm{O}_{2}^{\bullet-}\right]\right) \times\left(\frac{1}{1+K_{a}\left[H^{+}\right]^{-1}}\right) \\
& R_{\mathrm{HO}_{2}^{-}}= \\
& \left(-R_{U V, \mathrm{H}_{2} \mathrm{O}_{2}}-k_{2}\left[^{\bullet} \mathrm{OH}\right]\left[\mathrm{H}_{2} \mathrm{O}_{2}\right]-k_{3}\left[^{\bullet} \mathrm{OH}\right]\left[\mathrm{HO}_{2}^{-}\right]-k_{4}\left[\mathrm{HO}_{2}^{\bullet}\right]\left[\mathrm{H}_{2} \mathrm{O}_{2}\right]-k_{5}\left[\mathrm{O}_{2}^{\bullet-}\right]\left[\mathrm{H}_{2} \mathrm{O}_{2}\right]+\right. \\
& \left.k_{8}\left[{ }^{\bullet} \mathrm{OH}\right]^{2}+k_{9}\left[\mathrm{HO}_{2}^{\bullet}\right]^{2}+k_{11}\left[\mathrm{HO}_{2}^{\bullet}\right]\left[\mathrm{O}_{2}^{\bullet-}\right]\right) \times\left(\frac{1}{1+K_{a}^{-1}\left[H^{+}\right]}\right) \\
& R_{\mathrm{HO}_{2}^{*}}=k_{2}\left[^{\bullet} \mathrm{OH}\right]\left[\mathrm{H}_{2} \mathrm{O}_{2}\right]+k_{3}\left[^{\bullet} \mathrm{OH}\right]\left[\mathrm{HO}_{2}^{-}\right]-k_{4}\left[\mathrm{HO}_{2}^{\bullet}\right]\left[\mathrm{H}_{2} \mathrm{O}_{2}\right]+k_{6}\left[\mathrm{O}_{2}^{\bullet-}\right]\left[\mathrm{H}^{+}\right]-k_{7}\left[\mathrm{HO}_{2}^{*}\right]- \\
& k_{9}\left[\mathrm{HO}_{2}^{\bullet}\right]^{2}-k_{10}\left[{ }^{\circ} \mathrm{OH}\right]\left[\mathrm{HO}_{2}^{\bullet}\right]-k_{11}\left[\mathrm{HO}_{2}^{\bullet}\right]\left[\mathrm{O}_{2}^{\bullet-}\right] \\
& R_{\mathrm{O}_{2}^{--}}=-k_{5}\left[\mathrm{O}_{2}^{\bullet-}\right]\left[\mathrm{H}_{2} \mathrm{O}_{2}\right]-k_{6}\left[\mathrm{O}_{2}^{\bullet-}\right]\left[\mathrm{H}^{+}\right]+k_{7}\left[\mathrm{HO}_{2}^{\bullet}\right]-k_{11}\left[\mathrm{HO}_{2}^{\bullet}\right]\left[\mathrm{O}_{2}^{\bullet-}\right]-k_{12}\left[{ }^{\bullet} \mathrm{OH}\right]\left[\mathrm{O}_{2}^{\bullet-}\right]
\end{aligned}
$$




$$
\begin{aligned}
& R_{T O C_{1}}=-k_{14}[\cdot O H]\left[\text { TOC }_{1}\right] \\
& R_{r^{(0)}}=k_{14}\left[{ }^{\circ} \mathrm{OH}\right]\left[\mathrm{TOC}_{1}\right] \\
& R_{\text {TOC }_{2}}=k_{15} r^{(0)}-k_{16}\left[^{\circ} \mathrm{OH}\right]\left[\mathrm{TOC}_{2}\right] \\
& R^{\circ}{ }_{\mathrm{OH}}=2 R_{U V, \mathrm{H}_{2} \mathrm{O}_{2}}-k_{2}\left[{ }^{\bullet} \mathrm{OH}\right]\left[\mathrm{H}_{2} \mathrm{O}_{2}\right]-k_{3}\left[^{\bullet} \mathrm{OH}\right]\left[\mathrm{HO}_{2}^{-}\right]+k_{4}\left[\mathrm{HO}_{2}^{\bullet}\right]\left[\mathrm{H}_{2} \mathrm{O}_{2}\right]+k_{5}\left[\mathrm{O}_{2}^{--}\right]\left[\mathrm{H}_{2} \mathrm{O}_{2}\right]- \\
& k_{8}\left[{ }^{\circ} \mathrm{OH}\right]^{2}-k_{10}\left[^{\bullet} \mathrm{OH}\right]\left[\mathrm{HO}_{2}^{\cdot}\right]-k_{12}\left[^{\cdot} \mathrm{OH}\right]\left[\mathrm{O}_{2}^{\cdot-}\right]-k_{14}\left[\mathrm{TOC}_{1}\right]\left[\left[^{\bullet} \mathrm{OH}\right]-k_{16}\left[\mathrm{TOC}_{2}\right]\left[{ }^{\circ} \mathrm{OH}\right]\right.
\end{aligned}
$$

In these equations $\mathrm{TOC}_{1}$ and $\mathrm{TOC}_{2}$ are the TOC of the PVA and the PVA monomer, respectively. Radiation balance equations have to be developed and incorporated in the kinetic expressions shown in Equations (4.98), (4.99), and (4.105).

\subsubsection{Radiation balance}

Due to the small annular space in the laboratory-scale photoreactor, the uniform light distribution is considered throughout the photoreactor. Therefore, by employing the radiation balance, the LVREA is achieved based on the beer-Lambert law as follows:

$$
\begin{aligned}
& e_{\lambda}^{a}= \\
& I_{O}\left(\frac{\varepsilon_{\mathrm{H}_{2} \mathrm{O}_{2}\left[\mathrm{H}_{2} \mathrm{O}_{2}\right]+\varepsilon_{\mathrm{HO}_{2}^{-}}\left[\mathrm{HO}_{2}^{-}\right]}}{\varepsilon_{\mathrm{H}_{2} \mathrm{O}_{2}}\left[\mathrm{H}_{2} \mathrm{O}_{2}\right]+\varepsilon_{\mathrm{HO}_{2}^{-}}\left[\mathrm{HO}_{2}^{-}\right]+\varepsilon_{\mathrm{TOC}}\left[\mathrm{TOC}_{1}\right]}\right)\left(1-e^{\left(-2.303 b\left(\varepsilon_{\mathrm{H}_{2} \mathrm{O}_{2}}\left[\mathrm{H}_{2} \mathrm{O}_{2}\right]+\varepsilon_{H O_{2}^{-}}\left[\mathrm{HO}_{2}^{-}\right]+\varepsilon_{T O C_{1}}\left[\mathrm{TOC}_{1}\right]\right)\right)}\right)
\end{aligned}
$$

where $I_{o}$, the intensity of the incident light, was calculated to be $1.97 \times 10^{-5}$ Einstein $\mathrm{L}^{-1} \mathrm{~s}^{-1}$, which is the light incident at $254 \mathrm{~nm}$ photon irradiance estimated from $14 \mathrm{~W}$ LP lamp over the 
photoreactor volume assuming $10 \%$ attenuation by the quartz sleeve and $33 \%$ efficiency for the LP lamp. The fraction shows the portion of the UV radiation absorption by hydrogen peroxide. Parameters $\varepsilon_{\mathrm{H}_{2} \mathrm{O}_{2}}, \varepsilon_{\mathrm{HO}_{2}^{-}}$, and $\varepsilon_{T O C_{1}}$ are the molar extinction coefficients of $\mathrm{H}_{2} \mathrm{O}_{2}\left(18.7 \mathrm{M}^{-1} \mathrm{~cm}^{-1}\right)$, $\mathrm{HO}_{2}^{-}\left(210 \mathrm{M}^{-1} \mathrm{~cm}^{-1}\right), \mathrm{TOC}_{1}\left(153.24 \mathrm{M}^{-1} \mathrm{~cm}^{-1}\right)$ at $254 \mathrm{~nm}$, respectively, and $b$ is the effective path length (annular space) in the photoreactor (Tarr, 2003; Ghafoori et al., 2012b). The LVREA is incorporated in the direct photolysis of $\mathrm{H}_{2} \mathrm{O}_{2}$ as follows:

$$
R_{U V, H_{2} O_{2}}=\varphi_{H_{2} O_{2}} e_{\lambda}^{a}
$$

The kinetic model including the radiation balance is incorporated to the mass balance as depicted in Figure 4.49.

\subsubsection{Mass balance}

The general mass balance equation is applied to the recirculating batch photoreactor. Under the well-stirred conditions and high recirculating flow rate (small conversion per pass), the rate of the change of the concentration in the tank could be written as follows (Ghafoori et al., 2012b):

$\frac{d C_{i}}{d t}=\frac{V_{R}}{V_{T}}\left(R_{i}\right)$

where $C_{i}, t, V_{R}, V_{T}$, and $R_{i}$ are the molar concentration of component $i$ in the reacting system, time, photoreactor volume, tank volume, and the molar reaction rate of the component $i$, respectively. Therefore, the dynamic model Equations (4.98) to (4.105) are substituted into Equation (4.108) for each species and the obtained set of stiff ordinary differential equations are solved simultaneously. 


\subsubsection{Model validation}

In order to validate the kinetic model and also to obtain the kinetic parameters, experimental runs were carried out at different $\mathrm{H}_{2} \mathrm{O}_{2}$ dosages $\left(300,500,700,900\right.$, and $1100 \mathrm{mg} \mathrm{L}^{-1}$ ). The initial PVA concentration was $20 \mathrm{mg} \mathrm{L}^{-1}$. The results are presented in Figure 4.50. As illustrated in Figure 4.50, a good agreement between model predictions and experimental data are achieved, as it is confirmed in Figure 4.51.

\subsubsection{Kinetic parameter estimation}

In order to find the optimal kinetic parameters, the parameter estimation was performed based on the following objective function (Ghafoori et al., 2012b):

$\min J(z)=\sum_{i=1}^{N}\left(\frac{T O C_{m, i}(z)}{T O C_{i}}-1\right)^{2}$

subject to the obtained dynamic model equations.

where $z=\left[k_{14}, k_{15}, k_{16}\right]^{T}$

Here, $J(z)$ is the objective function, $T O C_{m, i}(z)$ and $T O C_{i}$ are the model prediction and the experimental data for TOC, respectively, and $N$ is the number of data points. The kinetic parameter estimation was carried out for experimental runs in batch recirculation system as described in the previous section. Therefore, the kinetic parameters are estimated as follows:

$k_{14}=6.2 \times 10^{6} \pm 2.2 \times 10^{6} \mathrm{M}^{-1} \mathrm{~s}^{-1}$

$k_{15}=5.25 \times 10^{2} \pm 0.013 \times 10^{2} \mathrm{~s}^{-1}$ 
$k_{16}=1.23 \times 10^{6} \pm 0.12 \times 10^{6} \mathrm{M}^{-1} \mathrm{~s}^{-1}$

The obtained kinetic parameters could be used diretly for the kinetic modeling of the pilot-scale photoreactor as illustrated in Figure 4.49.

\subsubsection{CFD Modeling of the Pilot-Scale Photoreactor}

As illustrated in the scaling-up methodology (Figure 4.49) the kinetic parameters estimated in the first part using the laboratory-scale photoreactor could be applied directly to the kinetic model of the pilot-scale photoreactor operating in continuous mode. The 3D pilot-scale photoreactor geometry depicted in Figure 4.52 is composed of two concentric cylinders (the inner cylinder is a quartz sleeve in which the UV lamp is inserted). The photoreactor operates under a continuous, steady-state condition. Other assumptions are: incompressible fluid, isothermal condition, and constant physical properties of the solution similar to water. The photoreactor description and operating conditions for the continuous flow in the photoreactor are summarized in Table 3.2. The final number of grid cells was determined to be 13,276 cells after conducting a grid independence study. COMSOL Multiphysics (Version 3.5) was utilized to simulate the 3D fluid field within the cylindrical photoreactor. As mentioned earlier, CFD modeling of a photoreactor requires a transport-reaction coupling. Therefore, it needs simultaneous solution of the kinetic model coupled with radiation, momentum, and mass balance equations.

\subsubsection{Radiation balance}

As mentioned earlier, a new model for the photon distribution (radiant energy balance) in the 


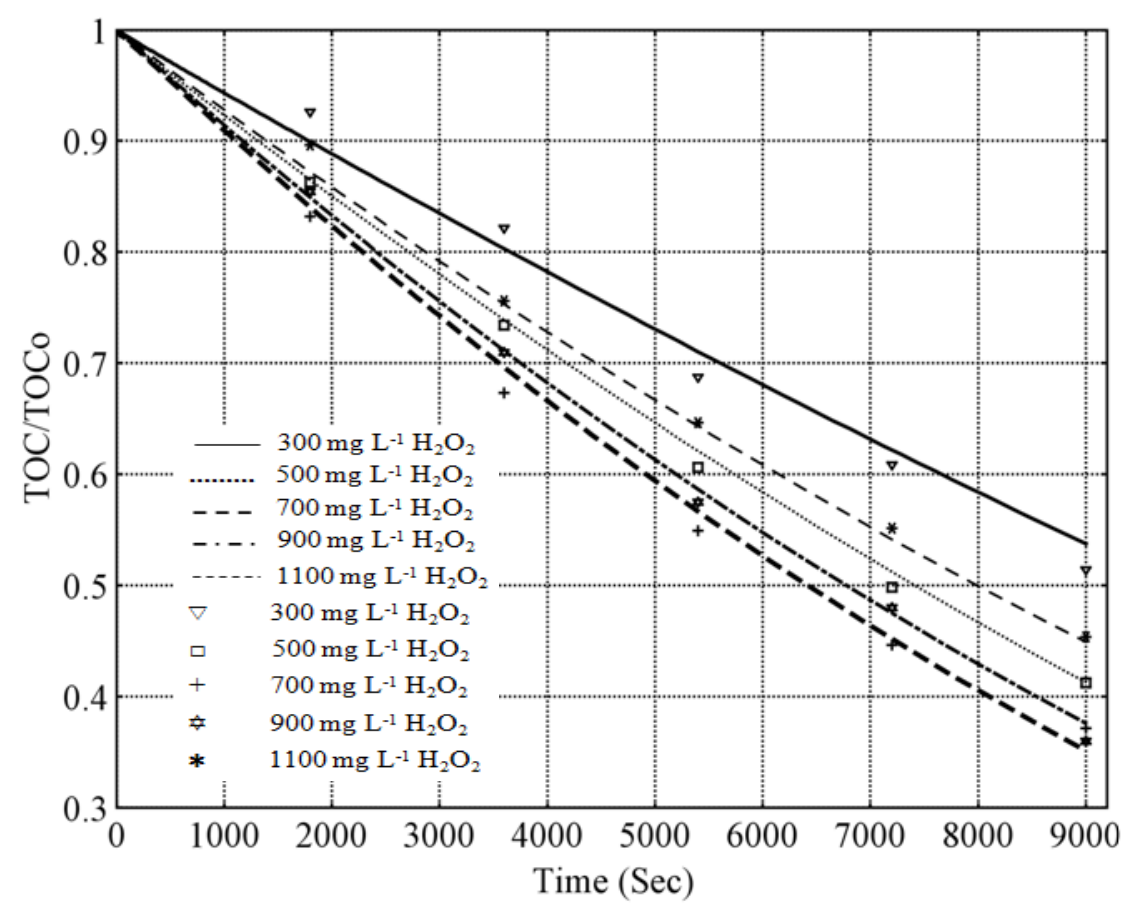

Figure 4.50. Experimental and predicted $\mathrm{TOC} / \mathrm{TOC}_{\mathrm{o}}$ concentration vs. time for $\mathrm{PVA}=20 \mathrm{mg} \mathrm{L}^{-1}$ in laboratory-scale photoreactor. Lines are model predictions and the symbols are corresponding experimental results. 


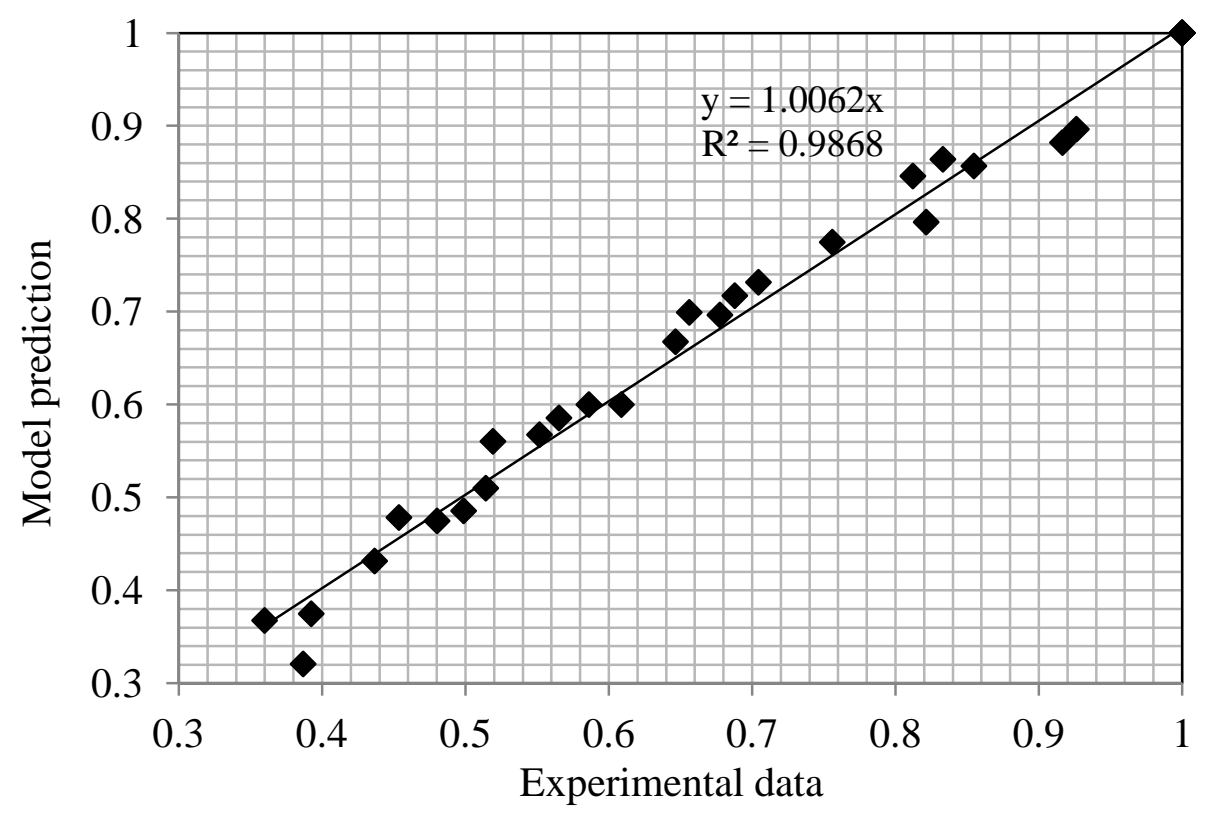

Figure 4.51. Kinetic model predictions vs. experimental results for TOC concentration of photodegradation of PVA by $\mathrm{UV} / \mathrm{H}_{2} \mathrm{O}_{2}$ at different dosage of $\mathrm{H}_{2} \mathrm{O}_{2}$ in laboratory-scale photoreactor $\left(300,500,700,900\right.$, and $\left.1100 \mathrm{mg} \mathrm{L}^{-1}\right)$. 
annular space is needed to determine LVREA $\left(e_{\lambda}^{a}\right)$ which has to be applied to the kinetic models. The radiation balance in the annular space of the pilot-scale photoreactor is different from the laboratory-scale photoreactor in which a uniform light distribution was assumed. In this case, a three-dimensional LVREA has to be taken into account (Cassano et al., 1995). The emission of the radiation from the cylindrical lamp is modeled using a three-dimensional source (TDS) with a volumetric emission model (Figure 4.53) based on the following assumptions:

- Emitters are uniformly distributed in the lamp extension given by its used length $\left(\mathrm{L}_{\mathrm{L}}\right)$ radius $\left(\mathrm{R}_{\mathrm{L}}\right)$;

- The radiation coming from each emitter is spherical, isotropic, and proportional to its extension;

- Each of the differential volumes of emission is transparent to the emission of its surroundings;

- The lamp is a perfect cylinder surrounded by mathematical surfaces without thickness. Hence, any bundle of radiation coming from inside does not change its intensity or direction when it crosses this boundary;

- The lamp is long enough; hence end effects in the lamp electrodes are neglected which indicates uniform emission produced by the lamp along its central axis; and

- Emission from the lamp is at steady state.

The radiation arrives at any point inside the photoreactor from a lamp that emits in all directions (volumetric emission in space). The lamp is separated from the reacting space by means of a quartz sleeve. The general radiant energy balance (Equation 2.30) is used with the following boundary condition (Equation 4.111) at the point of entrance $\left(S_{R}\right)$ : 


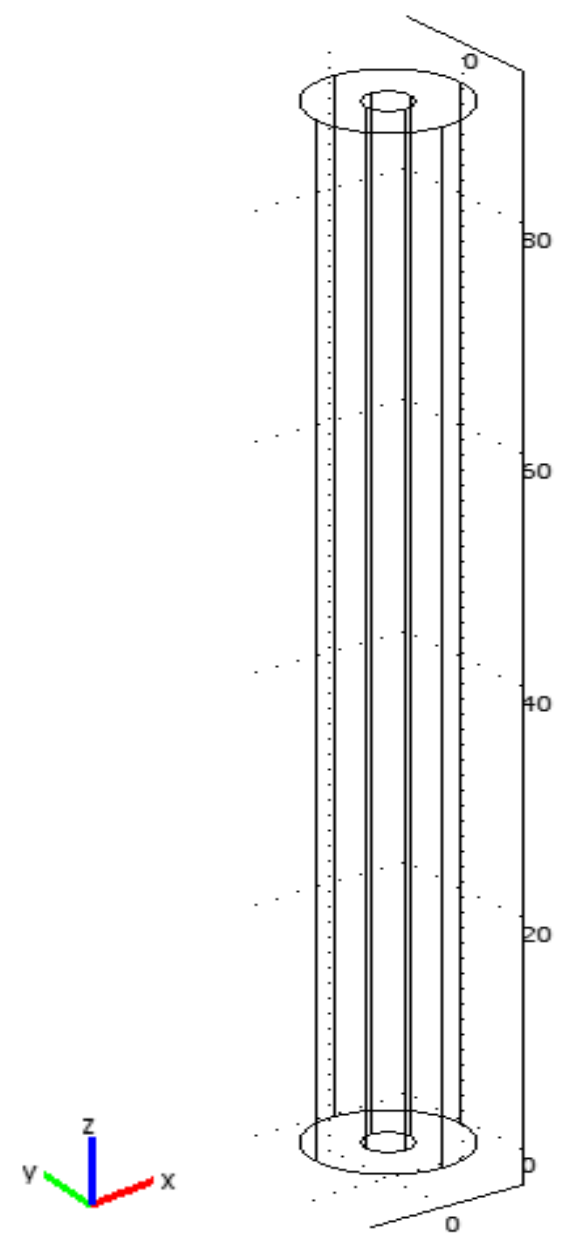

Figure 4.52. Pilot-scale photoreactor geometry (dimension are given in centimeters). 
Table 4.17. Pilot-scale photoreactor description in continuous flow.

\begin{tabular}{llc}
\hline Item & & Specification \\
\hline Photoreactor & Type & Annular \\
& Inner Diameter & $3 \mathrm{~cm}$ \\
& Outer Diameter & $9.72 \mathrm{~cm}$ \\
& Length & $90 \mathrm{~cm}$ \\
Lamp & Volume & $6 \mathrm{~L}$ \\
& Type & LP Hg UV \\
& Wavelength & $254 \mathrm{~nm}$ \\
& Length & $20.5 \mathrm{~cm}$ \\
Temperature & Output power & $13 \mathrm{~W}$ \\
Pressure & & $22^{\circ} \mathrm{C}$ \\
Operating Flow Rate & $1 \mathrm{~atm}$ \\
{$\left[\mathrm{PVA}_{\text {in }}\right.$} & & $0.1-0.5 \mathrm{~L} \mathrm{~min}^{-1}$ \\
{$\left[\mathrm{H}_{2} \mathrm{O}_{2}\right]_{\text {in }}$} & & $20 \mathrm{mg} \mathrm{L}^{-1}$ \\
\hline
\end{tabular}




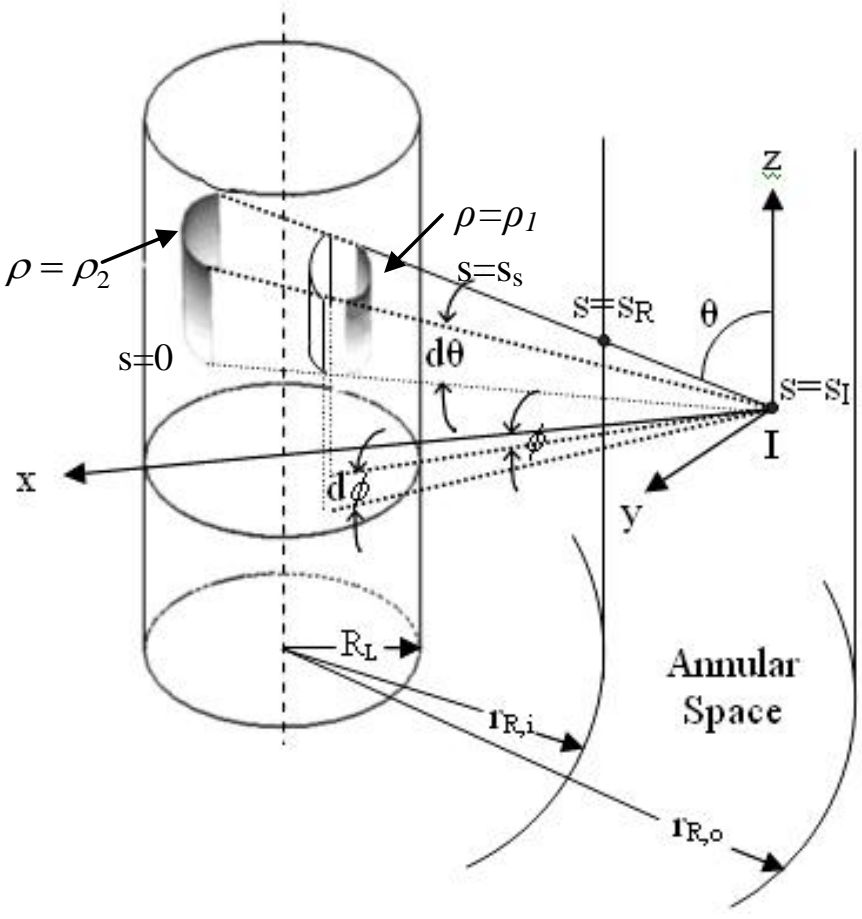

Figure 4.53. Schematic diagram of a three-dimensional source with volumetric emission model (Adapted by Cassano et al., 1995). 
$\frac{d I_{\lambda, \Omega}}{d s}(s, t)+\underbrace{\mu_{\lambda}(s, t) I_{\lambda, \Omega}(s, t)}_{\text {Absorption }}+\underbrace{\sigma_{\lambda}(s, t) I_{\lambda, \Omega}(s, t)}_{\text {Out-scatteing }}=\underbrace{\frac{\sigma_{\lambda}(s, t)}{4 \pi} \int_{\Omega^{\prime}=4 \pi} p_{\lambda}\left(\Omega^{\prime} \rightarrow \Omega\right) I_{\lambda, \Omega}(s, t) \mathrm{d} \Omega^{\prime}}_{\text {In-scattering }}$

$I(x, \theta, \phi, t)=I_{o}(\theta, \phi, t)$

where $I$ is the specific intensity, $x$ is the position inside the photoreactor, $\theta$ and $\phi$ are arbitrary directions inside the photoreactor, and $t$ is time. The boundary condition at the point of entrance at steady radiation is achieved by the following equation (Alfano and Cassano, 2009):

$I_{o}(\theta, \phi)=\frac{P_{L}}{4 \pi^{2} R_{L}^{2} Z_{L}} \cdot \frac{\left(R_{L}^{2}-r^{2} \sin ^{2} \phi\right)^{1 / 2}}{\sin \theta}$

where $P_{L}, R_{L}$, and $Z_{L}$ are the radiant power, radius, and length of the lamp, respectively. Integrating Equation 2.30 from the point of radiation entrance at the reactor wall $\left(s=s_{R}\right)$ to the point under consideration $(I)$ neglecting in and out-scattering, the following equation is obtained.

$I(x, \theta, \phi, t)=I_{o}(\theta, \phi, t) \exp \left[-\int_{s_{R}}^{s_{I}} \mu(s, t) \mathrm{d} s\right]$

where $\mu$ is the volumetric absorption coefficient. Integration of all possible directions of irradiation from the lamp volume of emission to the point $I$ over the solid angle of incidence ( $d \Lambda=\sin \theta d \theta d \phi)$ gives the value of the incident radiation $\left(G(x)=\int I(s, \Lambda) d \Lambda\right)$ at a point $x$ inside the reactor (Cassano et al., 1995).

$G(x, t)=\int_{\phi_{1}}^{\phi_{2}} \mathrm{~d} \phi \int_{\theta_{1}}^{\theta_{2}} \mathrm{~d} \theta \sin \theta I_{o}(\theta, \phi, t) \exp \left[-\int_{s_{R}}^{s_{I}} \mu(s, t) \mathrm{d} s\right]$

and the local volumetric rate of energy absorption $\left(e_{\lambda}^{a}(x, t)=\mu(x, t) G(x, t)\right)$ can be found subsequently as follows: 
$e_{\lambda}^{a}(x, t)=\mu(x, t) \int_{\phi_{1}}^{\phi_{2}} \mathrm{~d} \phi \int_{\theta_{1}}^{\theta_{2}} \mathrm{~d} \theta \sin \theta I_{o}(\theta, \phi) \exp \left[-\int_{S_{R}}^{S_{I}} \mu(s, t) \mathrm{d} s\right]$

Using the following change of coordinates and integrating from $s=0$ to $s=s_{s}$ (Figure 4.53) considering constant value for $\mu(s, t)$ give the LVREA as follows.

$d s=-d \rho$

$s=0 \quad \rho=\rho_{2}$

$s=s_{S} \quad \rho=\rho_{1}$

$e_{\lambda}^{a}(x, t)=\mu \int_{\phi_{1}}^{\phi_{2}} \mathrm{~d} \phi \int_{\theta_{1}}^{\theta_{2}} \mathrm{~d} \theta \sin \theta I_{o}(\theta, \phi) \exp \left[-\mu \Delta \rho_{\mathrm{s}}\right]$

where $s$ and $\rho$ are linear coordinates along the direction $\Lambda$ and distance from the radiation source to the point $I$, respectively (Figure 4.53 and Figure 4.54).The integration limits are obtained by writing the boundary surface of the radiation source (a cylinder) in spherical coordinates (Cassano et al., 1995):

$\rho^{2} \sin ^{2} \theta-2 \rho(\sin \theta \cos \phi) r+\left(r^{2}-R_{L}^{2}\right)=0$

Two solutions of this quadratic equation give the intersections of the $\rho$ coordinate with the front and rear parts of the lamp at any value of $\theta$ and $\phi$ :

$\rho_{1,2}=\frac{r \cos \phi \pm\left(r^{2} \cos ^{2} \phi-r^{2}+R_{L}^{2}\right)^{1 / 2}}{\sin \theta}$

Therefore $\Delta \rho$ in Equation 4.119 could be obtained as follows.

$\Delta \rho_{s}=\frac{2\left[r^{2} \cos ^{2} \phi-r^{2}+R_{L}^{2}\right]^{1 / 2}}{\sin \theta}$

Also from Figure 4.54 and Figure 4.55, the following equation could be written: 
$L_{L}-z=\rho_{1} \cos \theta_{1}$

Therefore, the integration limits for the Equation 4.119 will be achieved as follows (Cassano et al., 1995):

$\theta_{1}(\phi)=\tan ^{-1}\left\{\frac{r \cos \phi-\left[r^{2}\left(\cos ^{2} \phi-1\right)+R_{L}^{2}\right]^{\frac{1}{2}}}{\left(L_{L}-z\right)}\right\}$

$\theta_{2}(\phi)=\tan ^{-1}\left\{\frac{r \cos \phi-\left[r^{2}\left(\cos ^{2} \phi-1\right)+R_{L}^{2}\right]^{1 / 2}}{-z}\right\}$

The limits for independent variable $\phi$ can be found by imposing a restriction in the values of $\rho_{1}$ and $\rho_{2}$. At the limiting points, both intersections of the $\rho$ coordinate with the lamp boundary must coincide that means:

$\rho_{1}=\rho_{2}$

And from Equation 4.113:

$r^{2} \cos ^{2} \phi=r^{2}-R_{L}^{2}$

and subsequently:

$-\phi_{1}=\phi_{2}=\cos ^{-1}\left[\frac{\left(r^{2}-R_{L}^{2}\right)^{\frac{1}{2}}}{r}\right]$

Therefore, by determining all the unknown values and the integration limits, Equation 4.119 could be solved to achieve 3-dimensional LVREA $\left(e_{\lambda}^{a}(x, t)\right)$ through the photoreactor. LVREA is solved for the degradation of PVA $\left(20 \mathrm{mg} \mathrm{L}^{-1}\right)$ by the $\mathrm{UV} / \mathrm{H}_{2} \mathrm{O}_{2}$ process at different $\mathrm{H}_{2} \mathrm{O}_{2}$ dosages of through the annular space in the photoreactor as presented in Figure 4.56. As shown in this figure, the LVREA decreases by increasing the radius of the photorector. Also, the LVREA reduces by decreasing the concentration of $\mathrm{H}_{2} \mathrm{O}_{2}$ as it is considered as the main light ab- 


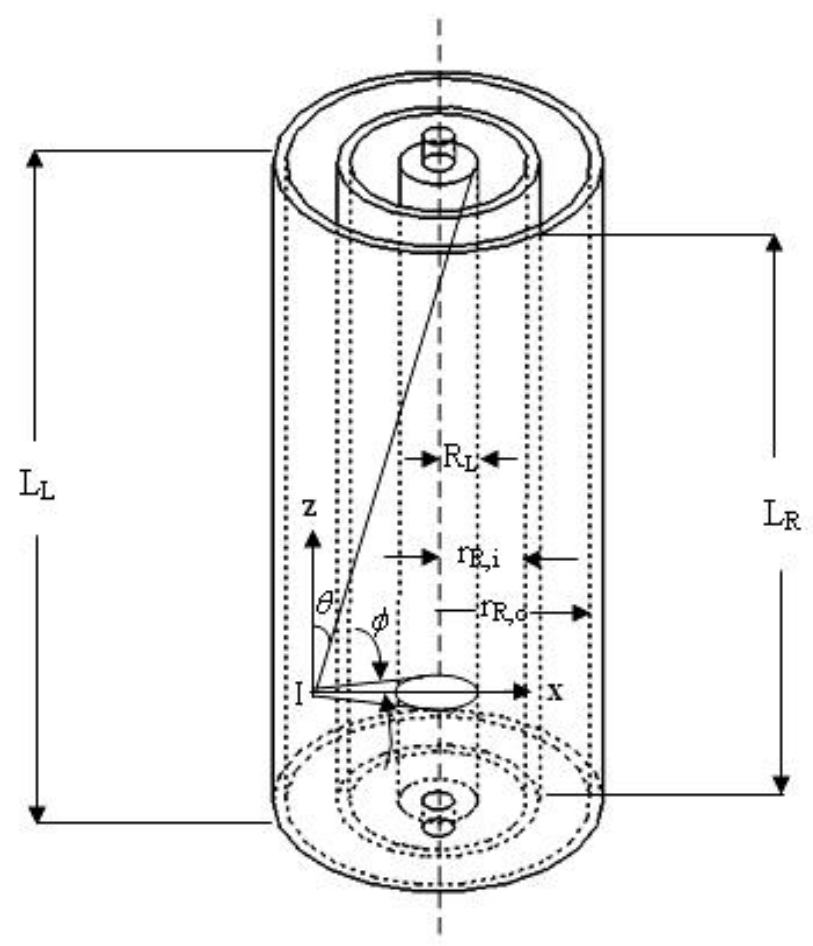

Figure 4.54. Continuous flow annular photoreactor (Adapted by Cassano et al., 1995). 


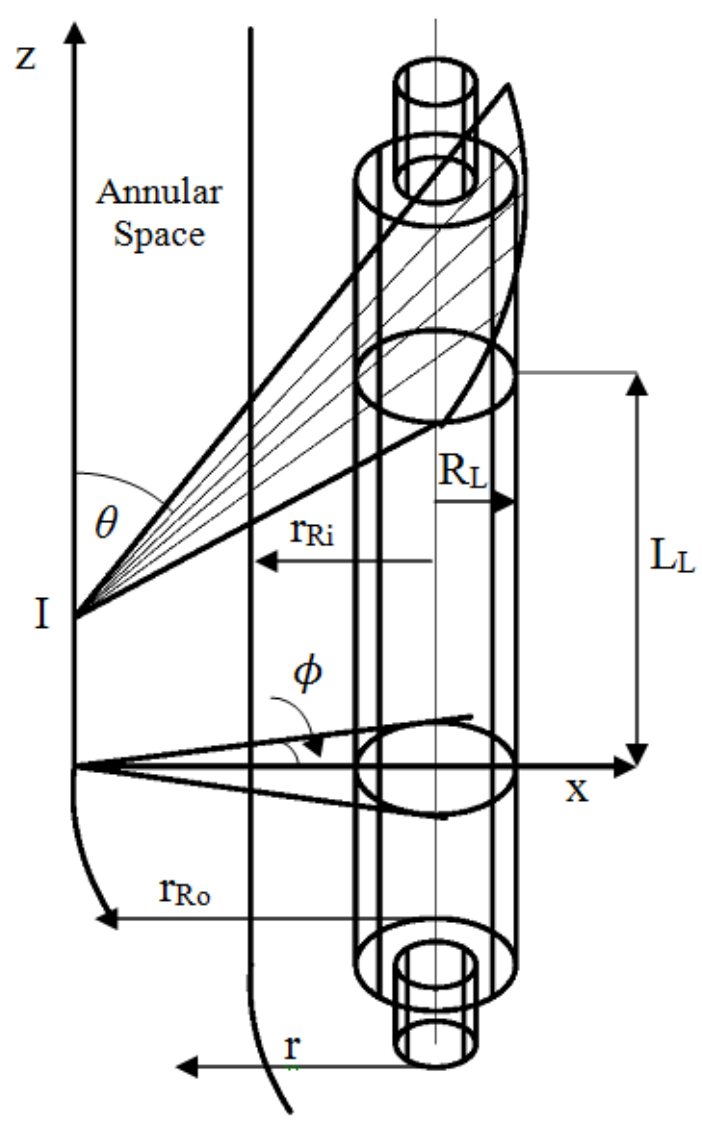

Figure 4.55. Limits of integration for the lamp (Adapted by Cassano et al., 1995). 
sorbing species in the solution. The LVREA will be used in the kinetic model incorporated in the mass balance equation.

\subsubsection{Momentum balance}

In order to determine the velocity profile for the laminar flow, steady-state incompressible Navier-Stokes equations are used for the momentum balance. Consequently, the governing equations for continuity and the momentum balances at constant density and viscosity could be described as follows:

$\nabla \cdot u=0$

$\rho(u . \nabla) u=\nabla \cdot\left[-p I+\eta\left(\nabla u+(\nabla u)^{T}\right)\right]+F$

where $\rho, u, \eta$, and $F$ are density, velocity vector, pressure, dynamic viscosity, and the body force, respectively. The boundary condition for the inlet is known value of the velocity, zero normal stress for the outlet, and no slip boundary conditions for all walls of the photoreactor and quartz sleeve. The velocity profile presented in Figure 4.57 is achieved by simultaneous solution of continuity and momentum equations along with their boundary conditions. As illustrated in Figure 4.57 (a) and (b) velocity profile is uniform along the photoreactor except the zero velocity near the walls which was expected based on the boundary conditions.

\subsubsection{Mass balance}

The continuity equation for steady-state mass balance for each species is given by the following equation: 


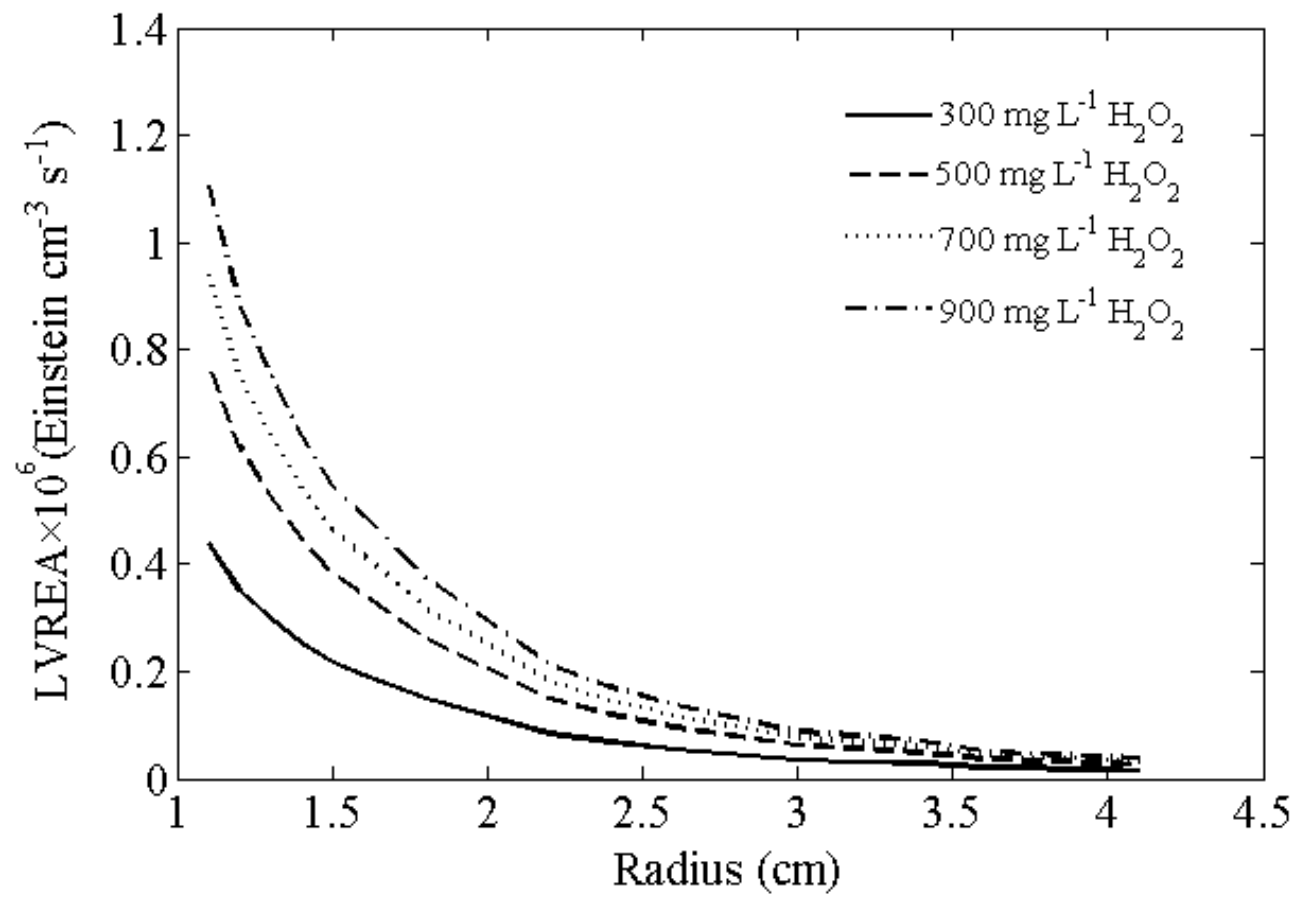

Figure 4.56. Local volumetric rate of energy absorption in the pilot-scale photoreactor. 
$\nabla \cdot\left(-D \nabla C_{i}\right)=-u \cdot \nabla C_{i}+R_{i}$

where $D$ is the diffusion coefficient, $C_{i}$ is the concentration of species $i, u$ is the velocity vector achieved from the momentum balance, and $R_{\mathrm{i}}$ is the reaction rate of species $i$. This governing equation is transport-reaction coupling which is carried out by directly exporting COMSOL Reaction Engineering Lab (Version 3.5), including the reaction formulas for all species present in Table 4.5, to the COMSOL Multiphysics Chemical Engineering Module to create the mass balance. In order to incorporate the 3-dimensional radiation balance, the COMSOL Multiphysics Chemical Engineering Module is connected to MATLAB program to solve the radiation balance. The velocity vector $(u)$ was determined in the previous section by solving the momentum balance equation. The boundary conditions for the mass balance are the inlet concentration of the species (20 mg L ${ }^{-1}$ PVA). The convective flux is considered at the outlet for all species. The concentration gradients on the photoreactor walls and the quartz sleeve are zero. The CFD simulation results for mass balance based on the TOC concentration of the PVA, convective flux, diffusive flux, and total flux for PVA are presented in Figure 4.58, Figure 4.59, Figure 4.60, and Figure 4.61, respectively. As is evident in Figure 4.58, the TOC concentration decreases as the solution passes along the photoreactor. $44.25 \%$ TOC removal was achieved in this process having flow rate of $0.1 \mathrm{~L} \mathrm{~min}^{-1}$ and $900 \mathrm{mg} \mathrm{L}^{-1} \mathrm{H}_{2} \mathrm{O}_{2}$. The higher residence time obviously would result in higher percent of TOC removal. The $\mathrm{H}_{2} \mathrm{O}_{2}$ concentration profile, convective flux, diffusive flux, and total flux for $\mathrm{H}_{2} \mathrm{O}_{2}$ are also presented in Figure 4.62, Figure 4.63, Figure 4.64, and Figure 4.65, respectively. As depicted in Figure 4.62, there is slight decrease in the concentration of $\mathrm{H}_{2} \mathrm{O}_{2}$ along the photoreactor as there is a chain of reactions for production and consumption of $\mathrm{H}_{2} \mathrm{O}_{2}$ (Table 4.1). 


\subsubsection{Validation of the Scaling-up Methodology}

In order to validate the CFD model predictions incorporating the mass, momentum, and 3dimensional radiation balances in the pilot-scale photoreactor, a set of experiments were carried out in continuous mode for different levels of $\mathrm{H}_{2} \mathrm{O}_{2}\left(300,500,700,900\right.$, and $\left.1100 \mathrm{mg} \mathrm{L}^{-1}\right)$ and different flow rates $\left(0.1,0.3\right.$, and $\left.0.5 \mathrm{~L} \mathrm{~min}^{-1}\right)$. The PVA concentration was $20 \mathrm{mg} \mathrm{L}^{-1}$ and there was no control on $\mathrm{pH}$ of the solution. The results based on the percent TOC removal for CFD model prediction and the experimental data in each case are presented in Figure 4.66. As is evident in this figure, there is a good agreement between the CFD model predictions and the experimental results confirming the reliability of the scaling-up methodology. Also, as is evident in this figure, increasing the dosage of $\mathrm{H}_{2} \mathrm{O}_{2}$ results in higher percent of the TOC removal up to the optimum level of $900 \mathrm{mg} \mathrm{L}^{-1}$ which above that the trend reverses. Also, there is slight decrease in the percent TOC removal by increasing the flow rate. As it was expected, higher residence time results in higher percent TOC removal.

\subsubsection{Conclusions}

A photoreactor scale-up methodology has been proposed to proceed from a laboratory photochemical reactor to a pilot-scale photoreactor without using experimentally adjusted parameters and costly changes of the photoreactor sizes. In order to employ this methodology, four conditions must be satisfied: (1) to have a detailed validated mechanism or kinetic scheme, (2) to have a validated detailed mechanistic kinetic model to find the intrinsic kinetic parameters, (3) to use the same spectral radiation $(\lambda)$ in both laboratory and pilot-scale photoreactors, (4) to employ and appropriately solve an accurate mathematical model to both photoreactors. For this 
a)

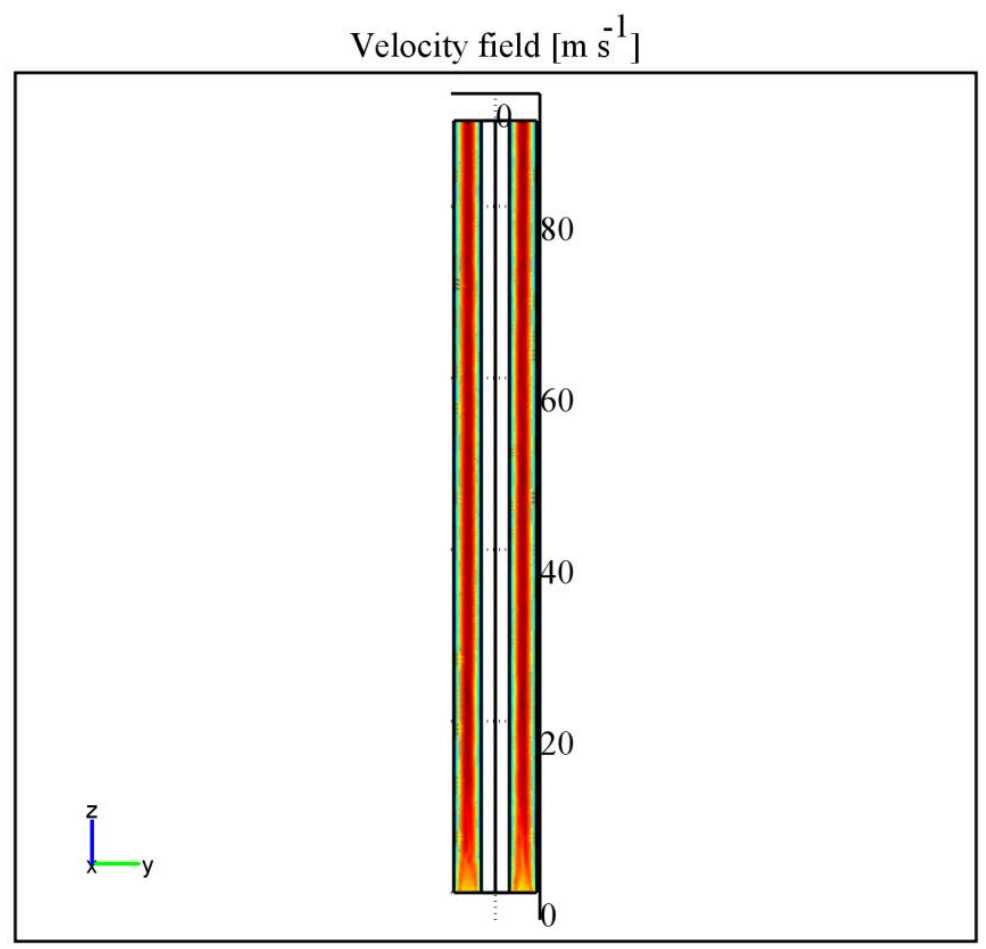

b)

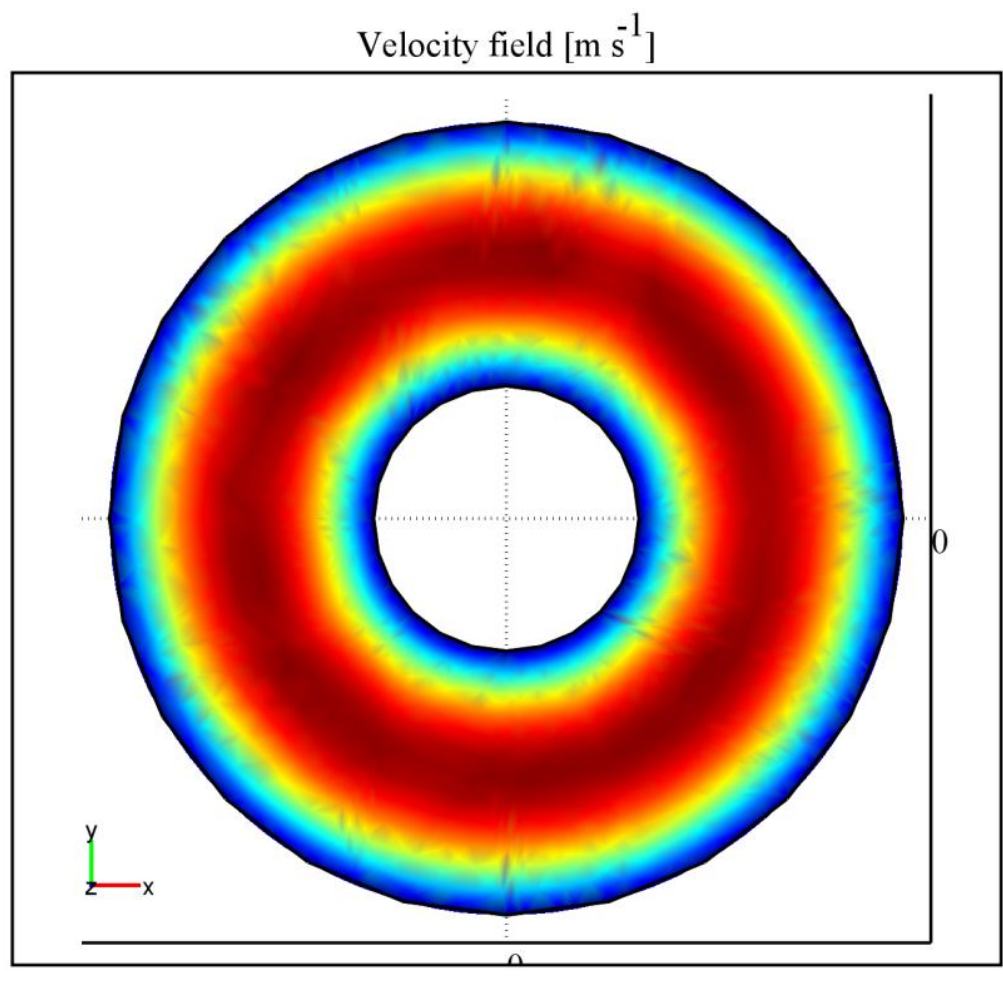

Max: 3.652e-4

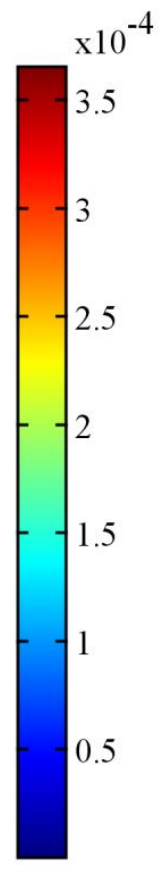

Min: $6.115 \mathrm{e}-32$

Max: $3.617 \mathrm{e}-4$

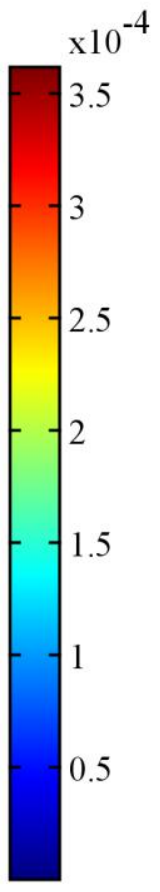

Min: $3.649 \mathrm{e}-31$

Figure 4.57. Velocity profile in (a) axial and (b) radial cross section of the pilot-scale photoreactor (PVA: $20 \mathrm{mg} \mathrm{L}^{-1}, \mathrm{H}_{2} \mathrm{O}_{2}: 900 \mathrm{mg} \mathrm{L}^{-1}$, and flow rate: $0.1 \mathrm{~L} \mathrm{~min}^{-1}$ ). 


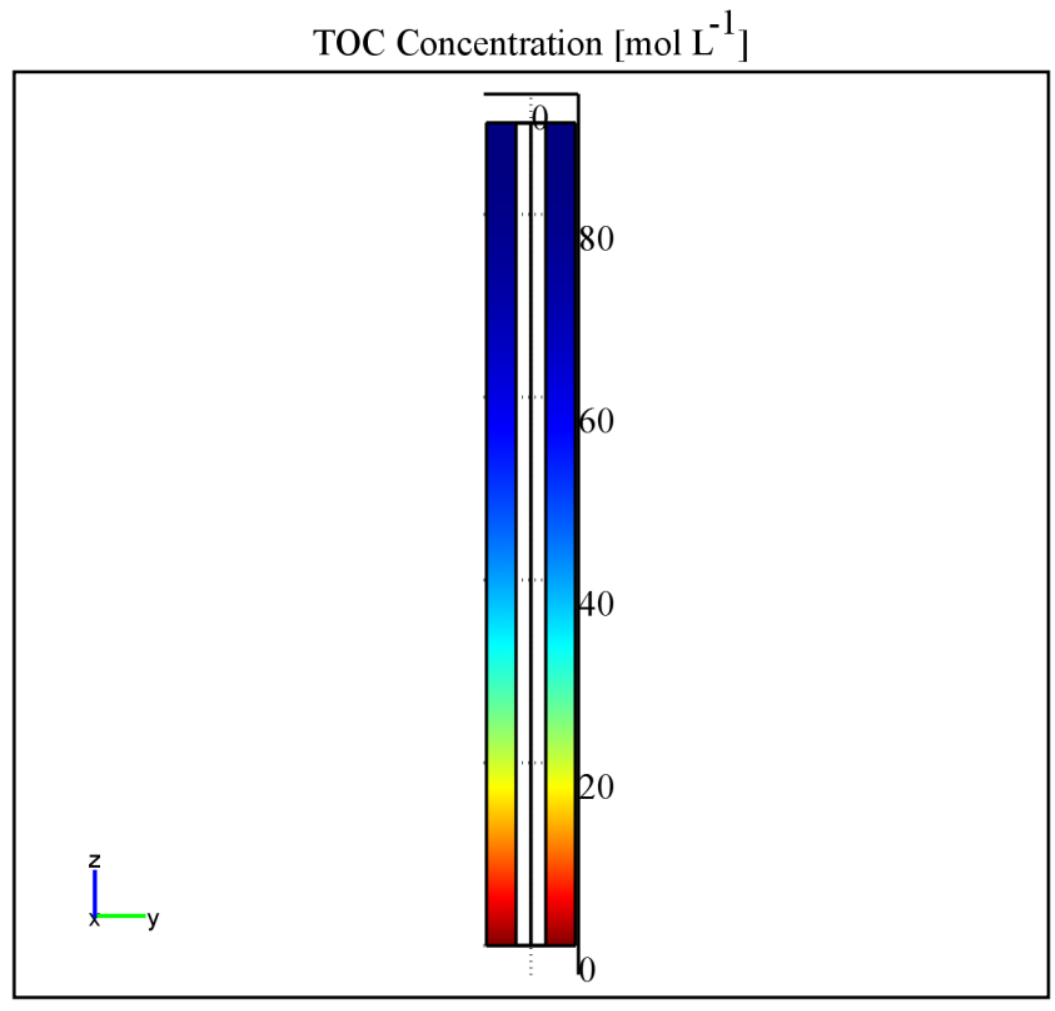

Max: 1.166e-3

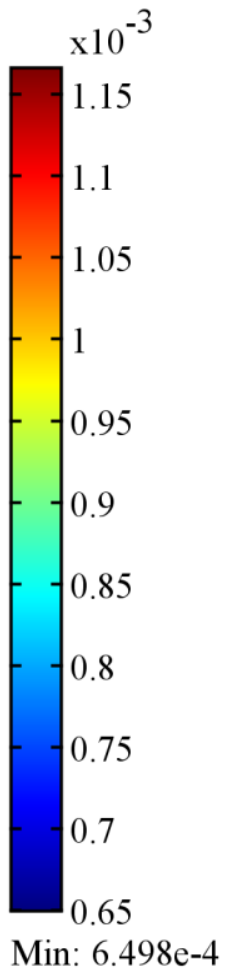

Figure 4.58. TOC concentration profile for PVA in the pilot-scale photoreactor (PVA: $20 \mathrm{mg} \mathrm{L}^{-}$ ${ }^{1}, \mathrm{H}_{2} \mathrm{O}_{2}: 900 \mathrm{mg} \mathrm{L}^{-1}$, and flow rate: $0.1 \mathrm{~L} \mathrm{~min}^{-1}$ ). 


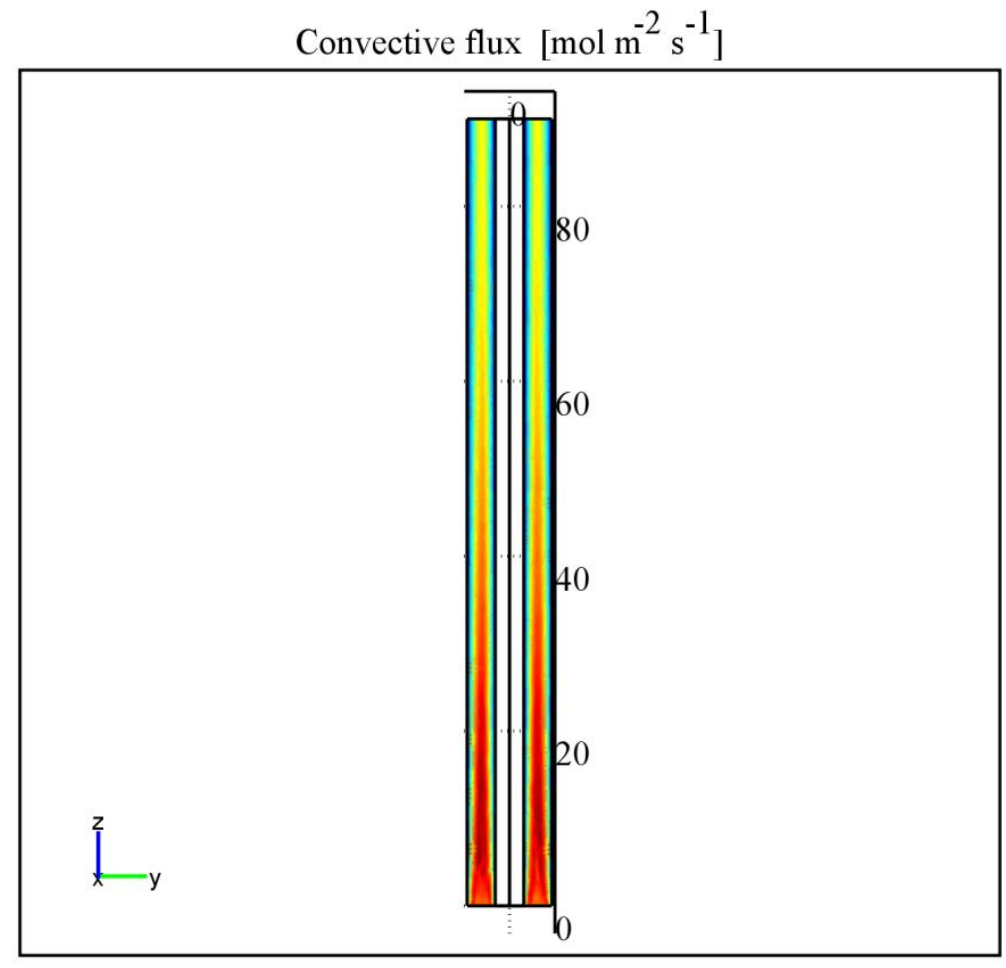

Max: 3.684e-7

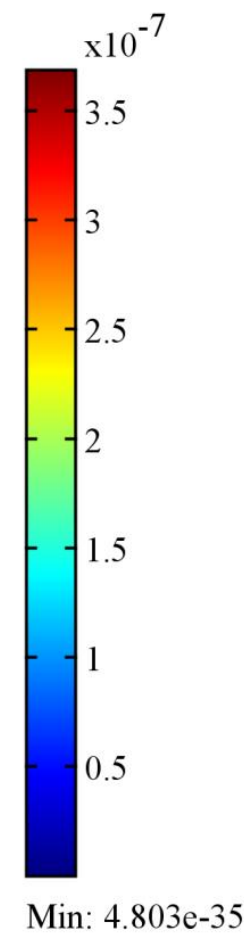

Figure 4.59. Convective flux for PVA in the pilot-scale photoreactor (PVA: $20 \mathrm{mg} \mathrm{L}^{-1}, \mathrm{H}_{2} \mathrm{O}_{2}$ : $900 \mathrm{mg} \mathrm{L}^{-1}$, and flow rate: $0.1 \mathrm{~L} \mathrm{~min}^{-1}$ ). 


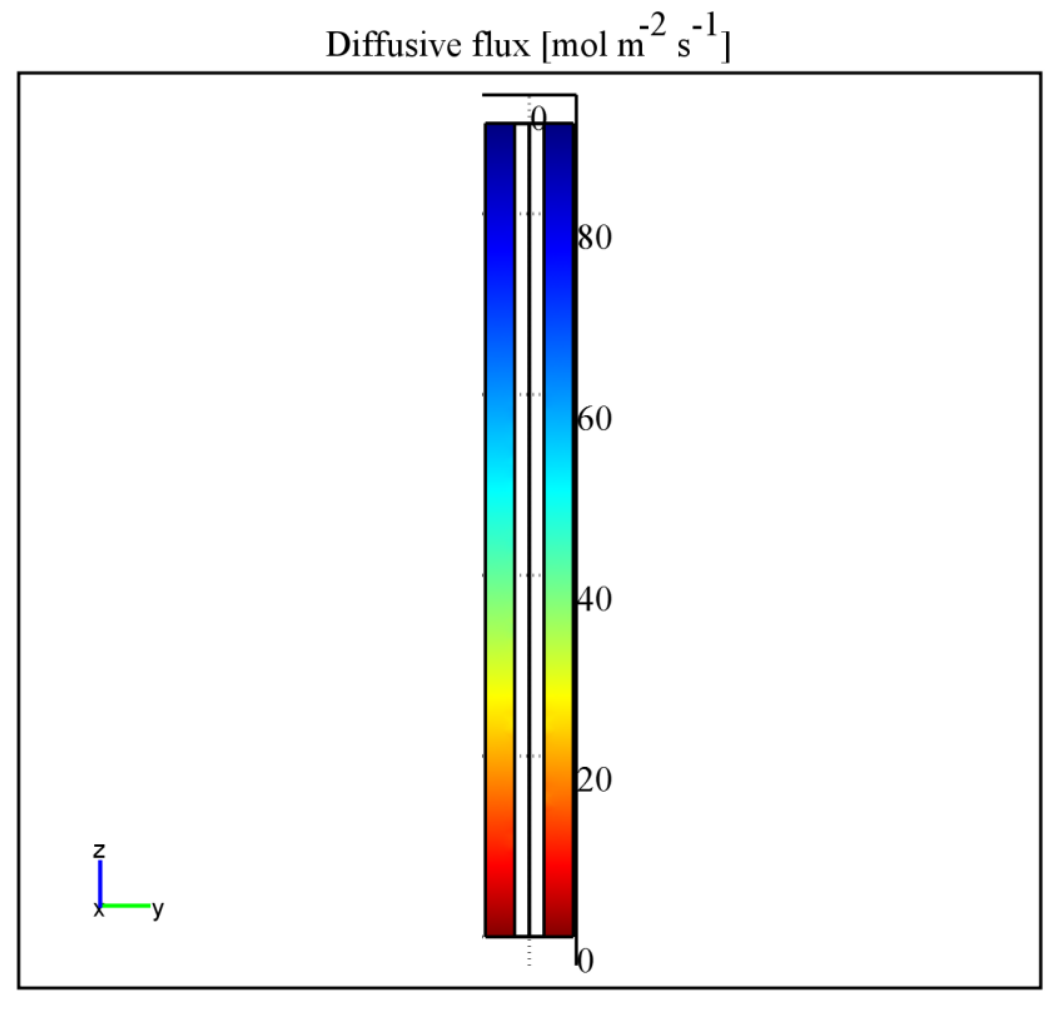

Max: $1.287 \mathrm{e}-5$

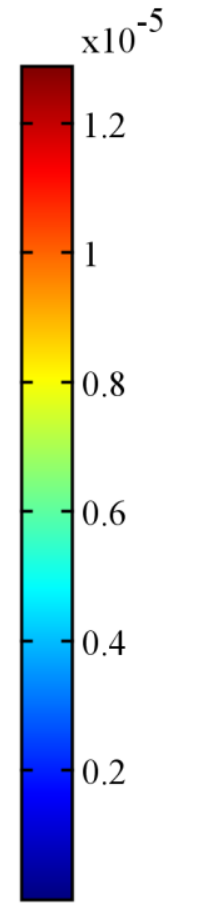

Min: $6.18 \mathrm{e}-11$

Figure 4.60. Diffusive flux for PVA in the pilot-scale photoreactor (PVA: $20 \mathrm{mg} \mathrm{L}^{-1}, \mathrm{H}_{2} \mathrm{O}_{2}: 900$ $\mathrm{mg} \mathrm{L}^{-1}$, and flow rate: $\left.0.1 \mathrm{~L} \mathrm{~min}^{-1}\right)$. 


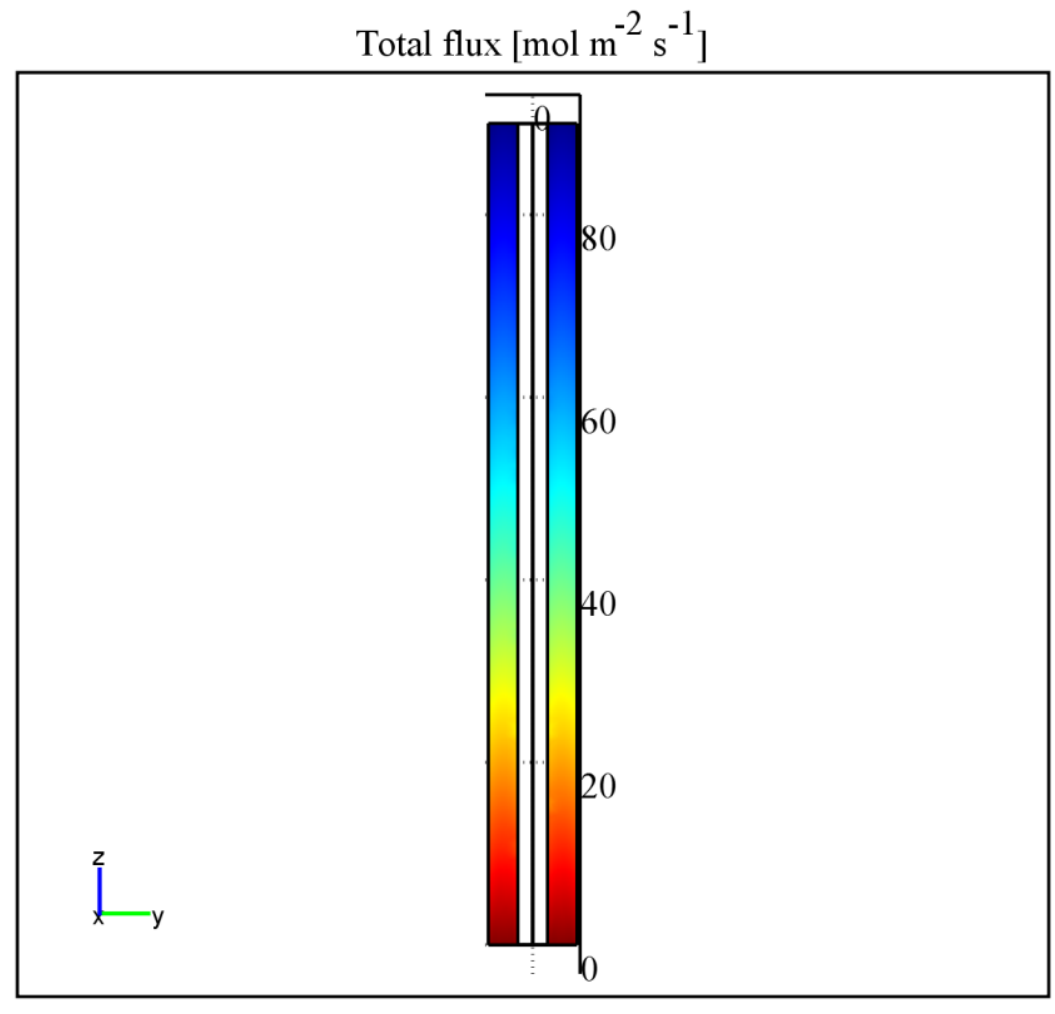

Max: $1.318 \mathrm{e}-5$

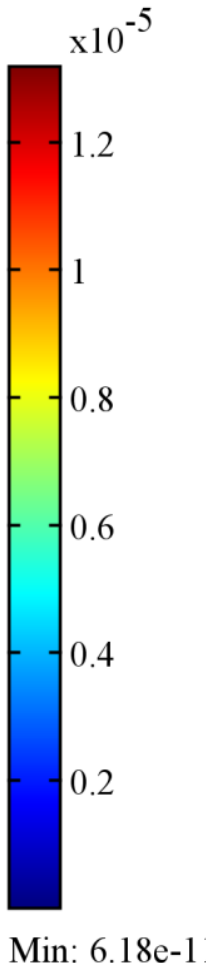

Figure 4.61. Total flux for PVA in the pilot-scale photoreactor (PVA: $20 \mathrm{mg} \mathrm{L}^{-1}, \mathrm{H}_{2} \mathrm{O}_{2}: 900 \mathrm{mg}$ $\mathrm{L}^{-1}$, and flow rate: $0.1 \mathrm{~L} \mathrm{~min}^{-1}$ ). 


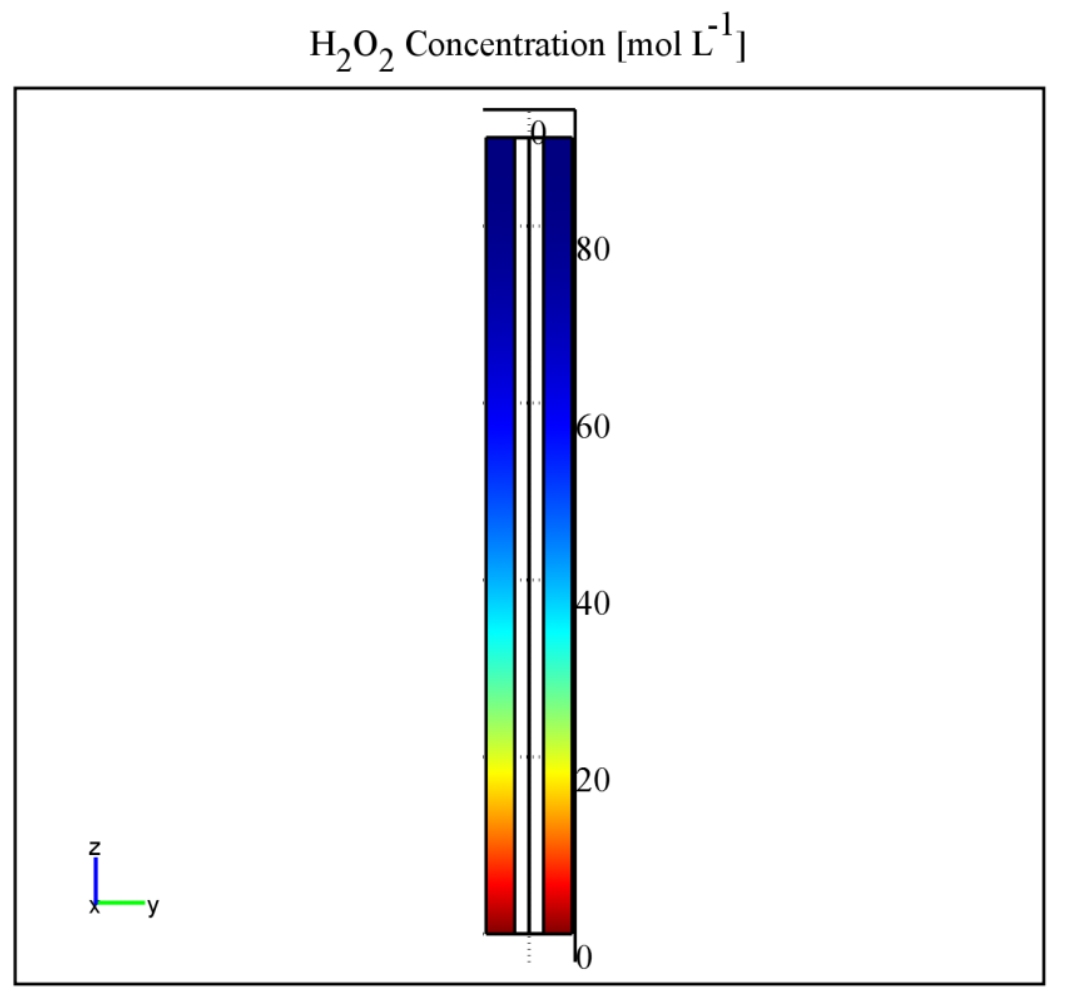

Max: 0.0265

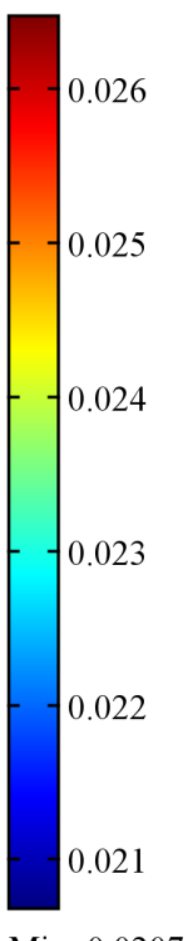

Min: 0.0207

Figure 4.62. Concentration profile for $\mathrm{H}_{2} \mathrm{O}_{2}$ in the pilot-scale photoreactor (PVA: $20 \mathrm{mg} \mathrm{L}^{-1}$, $\mathrm{H}_{2} \mathrm{O}_{2}: 900 \mathrm{mg} \mathrm{L}^{-1}$, and flow rate: $0.1 \mathrm{~L} \mathrm{~min}^{-1}$ ). 


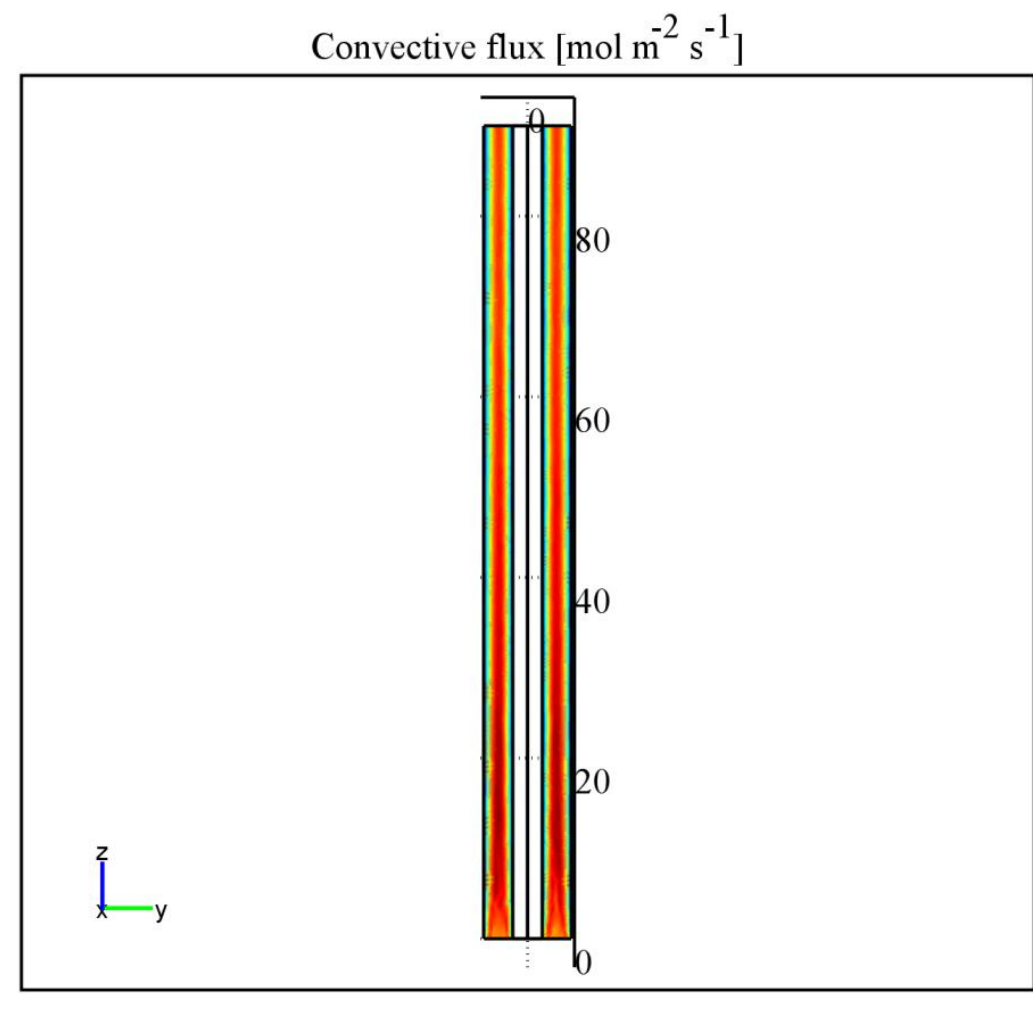

Max: $8.844 \mathrm{e}-6$

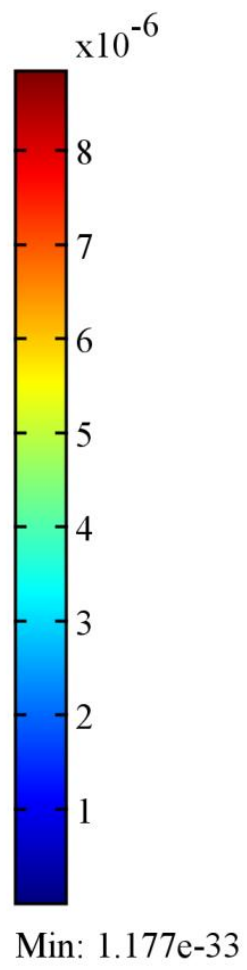

Figure 4.63. Convective flux for $\mathrm{H}_{2} \mathrm{O}_{2}$ in the pilot-scale photoreactor (PVA: $20 \mathrm{mg} \mathrm{L} \mathrm{L}^{-1}, \mathrm{H}_{2} \mathrm{O}_{2}$ : $900 \mathrm{mg} \mathrm{L}^{-1}$, and flow rate: $0.1 \mathrm{~L} \mathrm{~min}^{-1}$ ). 


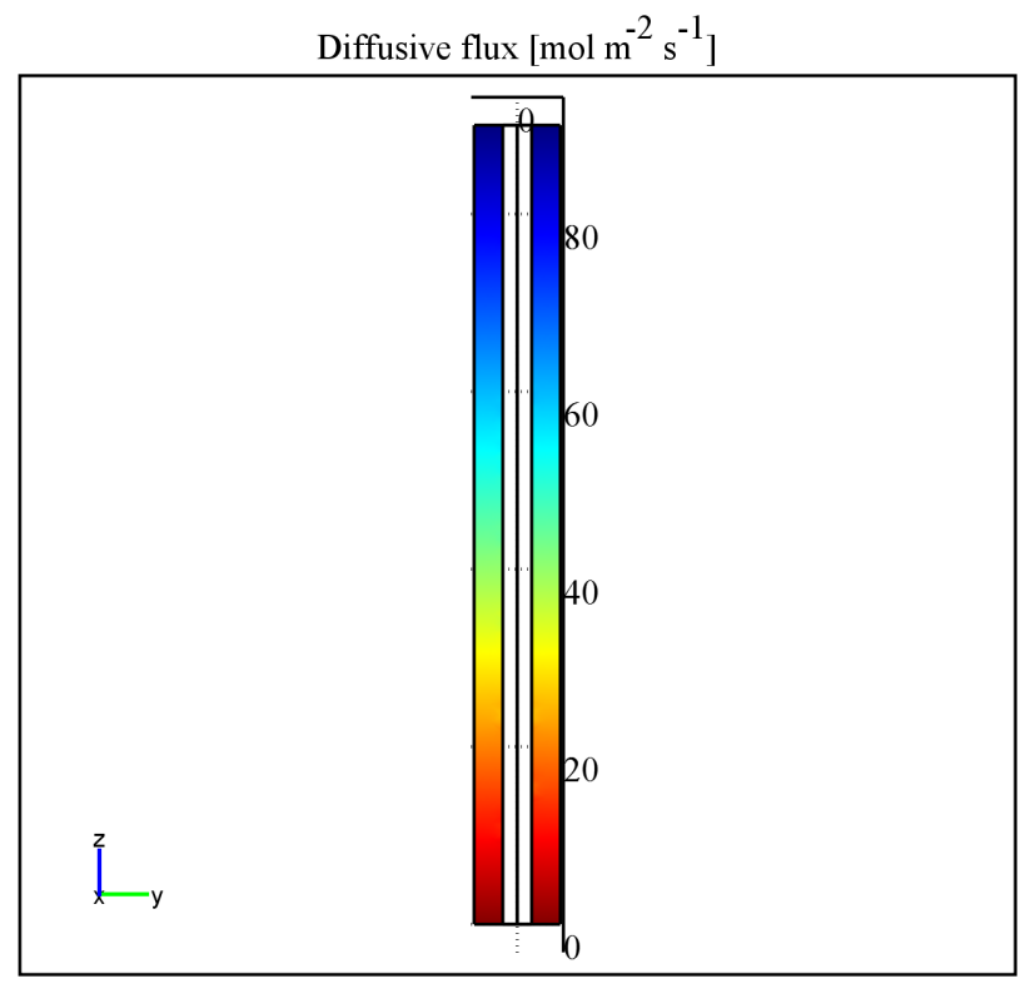

Max: $1.347 \mathrm{e}-4$

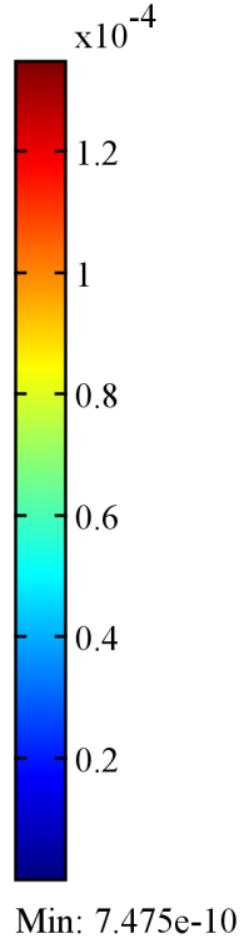

Figure 4.64. Diffusive flux for $\mathrm{H}_{2} \mathrm{O}_{2}$ in the pilot-scale photoreactor (PVA: $20 \mathrm{mg} \mathrm{L}^{-1}, \mathrm{H}_{2} \mathrm{O}_{2}$ : 900 $\mathrm{mg} \mathrm{L}^{-1}$, and flow rate: $0.1 \mathrm{~L} \mathrm{~min}^{-1}$ ). 


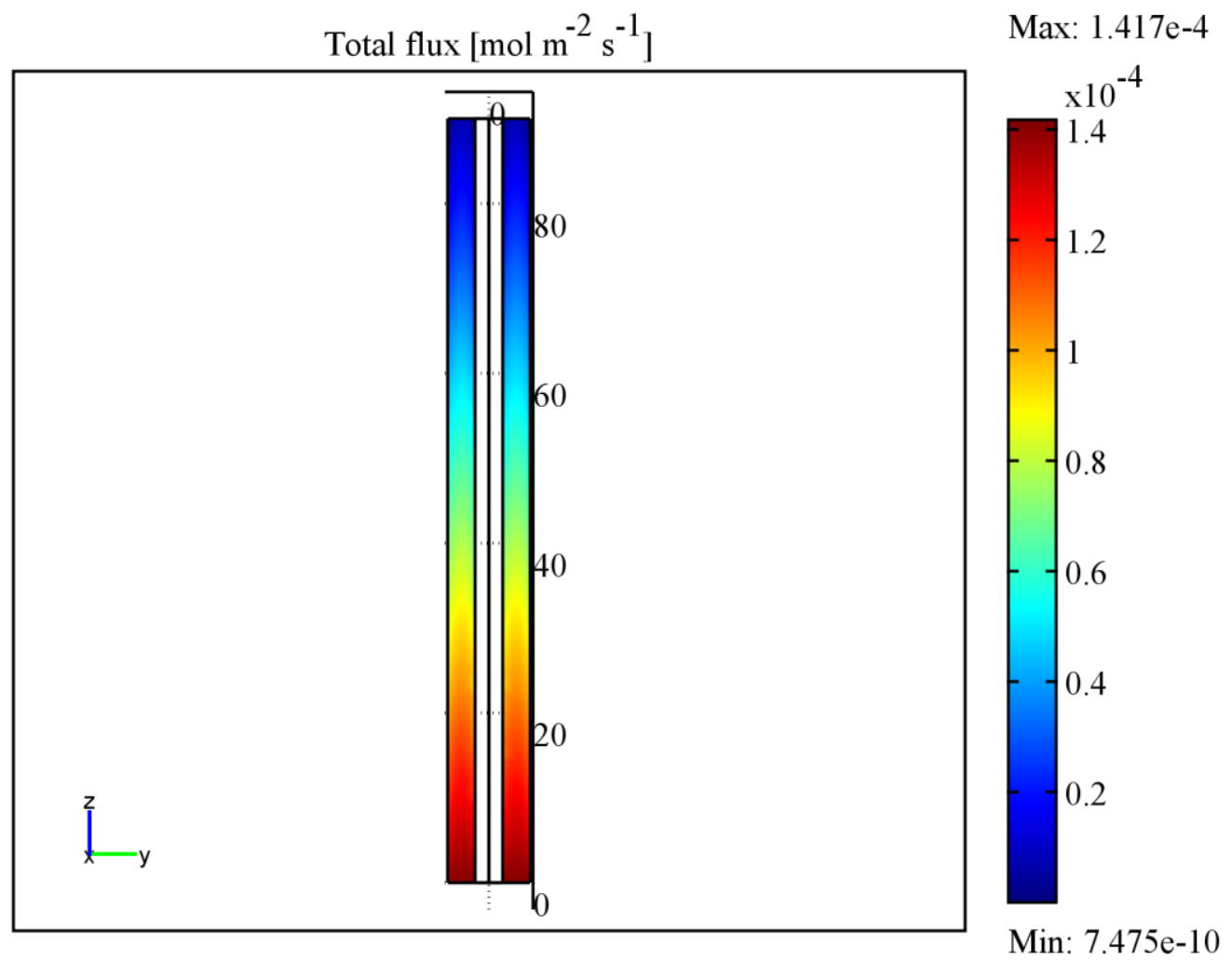

Figure 4.65. Total flux for $\mathrm{H}_{2} \mathrm{O}_{2}$ in the pilot-scale photoreactor (PVA: $20 \mathrm{mg} \mathrm{L}^{-1}, \mathrm{H}_{2} \mathrm{O}_{2}: 900 \mathrm{mg}$ $\mathrm{L}^{-1}$, and flow rate: $0.1 \mathrm{~L} \mathrm{~min}^{-1}$ ). 


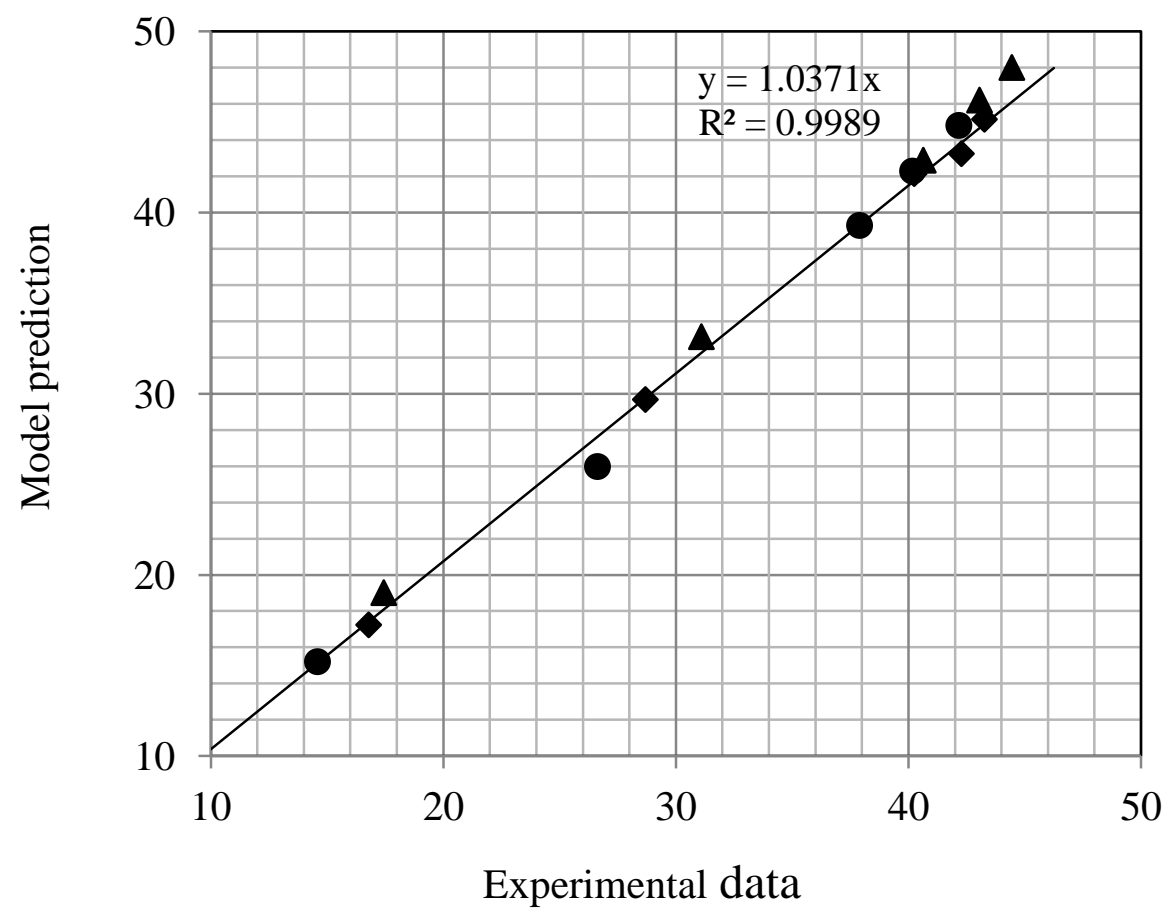

Figure 4.66. CFD Model prediction vs. experimental data for percent TOC removal in the pilotscale photoreactor at different $\mathrm{H}_{2} \mathrm{O}_{2}$ dosage $\left(300,500,700,900,1100 \mathrm{mg} \mathrm{L}^{-1}\right)$ and different inlet flow rate $\left(\boldsymbol{\wedge}: 0.1 \mathrm{~L} \mathrm{~min}^{-1}, \triangleleft: 0.3 \mathrm{~L} \mathrm{~min}^{-1}\right.$, and $\left.\bullet: 0.5 \mathrm{~L} \mathrm{~min}^{-1}\right)$. 
purpose, a laboratory batch recirculation system was used to achieve the kinetic parameters incorporating all important parameters influencing the photochemical reaction of PVA. Obtained kinetic parameters are independent of the photoreactor geometry and operation mode that can be used to predict the performance of the pilot-scale photoreactor with different geometry and mode of operation. The 3-dimensional radiation model was incorporated in the mass balance to model the pilot-scale photoreactor. The CFD model development was used to simulate the results of the proposed model in continuous flow. A good agreement between CFD simulation results and experimental data confirmed the reliability of the scaling-up methodology. 


\section{Chapter 5}

\section{CONCLUSIONS AND RECOMMENDATIONS}

\subsection{Conclusions}

The contribution of water-soluble polymers to the ever-increasing problem of water pollution has been a major concern. Most of the techniques currently applied to degrade these types of polymers have been found either inefficient or hazardous to the environment. AOTs have been recently applied as an effective method to degrade this type of polymers. However, only few studies are available in the open literature about the degradation and mineralization of polymers by AOTs. Also, due to the complexity of degrading high molecular weight compounds there is lack of information in literature in studying the degradation kinetics of water-soluble polymers using lumped parameters such as total organic carbon (TOC). Most of the established investigations in polymer degradation kinetics have centered on determining the rate of change of average molecular weight by thermal degradation. Also, the CFD modeling of AOTs and especially CFD modeling of the pilot-scale photoreactors considering 3D LVREA are scarce in literature.

In this study, the effectiveness of the photodegradation process using different methods of AOTs was investigated in a laboratory-scale batch recirculation system by advanced experimental design methods. Response surface methodology using Central Composite Design and BoxBehnken Design combined with quadratic programming is used for the experimental design, statistical analysis, and optimization of the process. The effects of different operating conditions on the process efficiency were investigated and statistically analyzed. The optimum operating 
conditions to maximize process efficiency were determined in each case using the numerical techniques. A detailed mechanistic kinetic model for the photodegradation of water-soluble polymers by different methods of AOTs is developed. Continuous distribution kinetics is applied to establish the kinetic model for the photodegradation of the polymers in aqueous solution. The proposed kinetic models are validated at different influential operating conditions using the results of the experimental design. A good agreement between the model predictions and the experimental results confirm the reliability of the proposed kinetic models. A methodology was developed in order to scale-up the laboratory-scale photoreactor to the larger scale photoreactor. In the scaling-up methodology, the intrinsic kinetics is determined firstly in the laboratory-scale photoreactor. Secondly, the obtained kinetic expressions are directly incorporated in the mass balance for the pilot-scale photoreactor. The CFD development was employed to simulate the results of the scaling-up methodology. The procedure was satisfactory validated by comparing the simulation results with the experimental data. Following are the main conclusions of the thesis in detail:

1. The mathematical kinetic model developed to predict the number average molecular weight for photodegradation of water-soluble polymers by $\mathrm{UV} / \mathrm{H}_{2} \mathrm{O}_{2}$ showed satisfactory results when compared to the experimental results. The kinetic rate constants were determined by nonlinear parameter estimation using an optimization algorithm. The optimal values of the kinetic parameters that are independent of the reactor size and configuration can be considered as the starting point for designing commercial scale photoreactors.

2. The response surface methodology using $\mathrm{CCD}$ and quadratic programming showed reliable results for the photodegradation of $\mathrm{PEO}$ by the $\mathrm{UV} / \mathrm{H}_{2} \mathrm{O}_{2}$ process. Predicted results from the response functions were in good agreement with the experimental data confirming the reliability 
of the employed methodology. The optimum percent TOC removal (84\%) was found after 150 min at the operating conditions of $10 \mathrm{mg} \mathrm{L}^{-1} \mathrm{PEO}, 780 \mathrm{mg} \mathrm{L}^{-1} \mathrm{H}_{2} \mathrm{O}_{2}$, pH 3, and $0.5 \mathrm{~L} \mathrm{~min}^{-1}$ recirculation rate based on the developed quadratic model. A mathematical model was developed based on the mass balance of the main species present in water to predict the percent TOC removal of PEO. An excellent agreement between the model predictions and the experimental data confirmed the reliability of the predicted model. The estimated kinetic parameters using an optimization algorithm were also in the acceptable range of reported values in the open literature.

3. Advanced oxidation of PAA by the photo-Fenton-like process $\left(\mathrm{UV} / \mathrm{Fe}^{3+} / \mathrm{H}_{2} \mathrm{O}_{2}\right)$ in aqueous solution was investigated using Box-Behnken experimental design combined with RSM and quadratic programming. The statistical analysis of the results indicated satisfactory prediction of the system behavior by the developed model. The optimal operating conditions to achieve the maximum TOC removal rate (93.94\%) in the selected range was determined to be $22.80 \mathrm{mg} \mathrm{L}^{-1}$ PEO, $7 \mathrm{mg} \mathrm{L}^{-1} \mathrm{Fe}^{3+}, 1300 \mathrm{mg} \mathrm{L}^{-1} \mathrm{H}_{2} \mathrm{O}_{2}$, respectively. A detailed mathematical kinetic model for $\mathrm{UV} / \mathrm{Fe}^{3+} / \mathrm{H}_{2} \mathrm{O}_{2}$ process including detail reactions of advanced oxidation technologies, iron chlorocomplexes, inorganic anion radicals, and polymer cleavage was developed and validated experimentally. Kinetic parameter of the hydrogen abstraction from the polymer and also the model parameter of the photolysis of hydrogen peroxide were the most influential parameters on the percent TOC removal based on the results of the sensitivity analysis. CFD modeling incorporating rigorous kinetic rate expressions and governing transport equations were studied in a continuous flow and was verified experimentally. A good agreement between CFD simulation results and the experimental data confirmed the model adequacy.

4. The experimental design, statistical analysis, and optimization for the PAA photodegradation by photo-Fenton-like process was investigated in a batch recirculation system using two 
photoreactors in series. The effects of the initial concentrations of PAA and $\mathrm{H}_{2} \mathrm{O}_{2}: \mathrm{Fe}^{3+}$, $\mathrm{pH}$, and the recirculation rate on the percent TOC removal rate and the fitted values of "pseudo"-second order mineralization rate constants were studied using a four-factor three-level Box-Behnken experimental design combined with RSM and quadratic programming. The statistical analysis using ANOVA confirmed accuracy of the developed quadratic models. The optimal operating conditions to achieve the maximum TOC removal rate $(95.7 \%)$ in the selected range was determined to be $10 \mathrm{mg} . \mathrm{L}^{-1}$ PAA, $1600 \mathrm{mg} . \mathrm{L}^{-1} \mathrm{H}_{2} \mathrm{O}_{2}, 40 \mathrm{mg} . \mathrm{L}^{-1} \mathrm{Fe}^{3+}$, pH 3.42, and $10 \mathrm{~L} \cdot \mathrm{min}^{-1}$ recirculation rate, respectively. The "pseudo"-order rate constant at the optimum conditions for TOC removal was found to be $82 \mathrm{M}^{-1} \cdot \mathrm{s}^{-1}$. The obtained optimal operating conditions were also validated experimentally indicating the reliability of the developed models.

5. A methodology for scaling-up a photoreactor for the photodegradation of PVA using $\mathrm{UV} / \mathrm{H}_{2} \mathrm{O}_{2}$ process was proposed. In order to employ this methodology, four conditions must be satisfied: (1) to have a detailed validated mechanism or kinetic scheme, (2) to have a validated detailed mechanistic kinetic model to find the intrinsic kinetic parameters, (3) to use the same spectral radiation $(\lambda)$ in both laboratory and pilot-scale photoreactors, (4) to employ and appropriately solve an accurate mathematical model to both photoreactors. In this procedure the intrinsic kinetic parameters were determined using a laboratory-scale batch recirculating photoreactor. The obtained kinetic parameters were used to predict the performance of the pilotscale continuous flow through photoreactor without any adjustable parameter. Three-dimensional radiation balance and differential mass balance incorporating the detailed kinetic model were developed using CFD model. The simulation results were compared to a set of experimental data carried out at different $\mathrm{H}_{2} \mathrm{O}_{2}$ dosage and inlet flow rate. Good agreement between the simulation results and the experimental data confirmed the reliability of the scaling-up methodology. 


\subsection{Recommendations for Future Work}

Although this study highlighted new insights into the polymer degradation by advanced oxidation technologies there are still some areas for future consideration as follows:

1. To investigate the molecular weight distribution during the photodegradation of the polymers using AOTs. It can provide another insight into the photodegradation process.

2. It is recommended to study the viscosity average molecular weight changes for photodegradation of the polymers in aqueous solution. Modeling the viscosity average molecular weight could be also carried out using moment operation.

3. To study polymer degradation and kinetic modeling of the polymer degradation by the other methods of AOTs such as photocatalysis by $\mathrm{TiO}_{2}$ and $\mathrm{ZnO}$ in order to compare the photodegradation efficiency of different AOTs.

4. It is suggested to use different UV lamps with various intensities to compare the effect of the light intensity on polymer degradation because light intensity has significant effect on the photodegradation effectiveness. However, higher light intensity may lead to the recombination of carbon-peroxyl radicals and formation of polymers

5. To investigate the AOTs for the polymer degradation as a pretreatment method to biological degradation and optimization of the integrated process in terms of all involved parameters. Integration of photodegradation and biodegradation makes the whole process cost-effective.

6. To study the CFD modeling of the multiphase flow for the pilot-scale photoreactor operating in turbulent regime as the information on CFD modeling of multiphase flow photoreactors in turbulent regime is scarce in the literature. 


\section{Nomenclature}

$\begin{array}{ll}A_{254} & \text { UV absorbance at } 254 \mathrm{~nm} \\ b & \text { UV path length in the photoreactor [cm] } \\ c_{p} & \text { Rotatable number of center point } \\ C_{i} & \text { Concentration of chemical species in water }[\mathrm{M}] \\ c_{T O C_{1}} & \text { TOC concentration of PEO }[\mathrm{M}] \\ d & \text { Degree of freedom } \\ D & \text { Diffusion coefficient }\left[\mathrm{m}^{2} / \mathrm{s}\right] \\ e & \text { Residuals } \\ e_{\lambda}^{a} & \text { Local volumetric rate of energy absorption }\left[\text { Einstein } \mathrm{L} \mathrm{s}^{-1}\right] \\ F_{M} & \text { Fraction of radiation that is absorbed by M } \\ I_{O} & \left.\text { UV light intensity [Einstein } \mathrm{cm}^{-3} \mathrm{~s}^{-1}\right] \\ J(z) & \text { Objective function } \\ k & \text { Factor number } \\ K_{a} & \text { Equilibrium constant } \\ k_{i} & \text { Rate constant }\left[\mathrm{M}^{-1} \mathrm{~s}^{-1}\right],\left[\mathrm{s}^{-1}\right] \\ k_{\mathrm{obs}} & \text { "pseudo"-second order rate constant }\left[\mathrm{M}^{-1} \mathrm{~s}^{-1}\right] \\ k_{\lambda} & \text { Volumetric absorption coefficient }\left[\mathrm{m}^{-1}\right] \\ l & \text { UV path length in the spectrophotometer }\left[\mathrm{cm}^{-1}\right] \\ N_{i} & \left.\text { Molar flux [mol } \mathrm{cm}^{-2} \mathrm{~s}^{-1}\right] \\ m & \text { Parameter indicating the shape of the scission fragment distribution } \\ m(n, t) & \text { Chain length distribution of monomer } \\ n & \text { Chain length } \\ \mathrm{n}_{\mathrm{c}} & \text { Number of center points } \\ N & \text { Total number of experiments } \\ & \end{array}$




\begin{tabular}{|c|c|}
\hline$p_{\lambda}$ & Phase function \\
\hline$p(n, t)$ & Chain length distribution of polymer \\
\hline$P_{n}$ & Polymer of chain length $n$ \\
\hline$P_{L}$ & Radiation power [Einstein $\mathrm{s}^{-1}$ ] \\
\hline$q_{i}$ & Flux of incident radiation in a photoreactor $\left[\mathrm{J} \mathrm{m}^{-2} \mathrm{~s}^{-1}\right]$ \\
\hline$r(n, t)$ & Chain length distribution of end radical \\
\hline$r^{(p)}$ & P-th order moment operation \\
\hline$R^{2}$ & Regression coefficient \\
\hline$R_{\text {adj }}^{2}$ & Adjusted regression coefficient \\
\hline$R_{i}$ & Reaction rate $\left[\mathrm{M}^{-1} \mathrm{~s}^{-1}\right]$ \\
\hline$R_{L}$ & Radius of the lamp [cm] \\
\hline$R_{n}^{\bullet}$ & Polymer radical of chain length $n$ \\
\hline $\operatorname{Re}$ & Reynolds number \\
\hline$s$ & Linear coordinate along the direction $\Omega[\mathrm{m}]$ \\
\hline$S$ & Number of chain scission per molecule \\
\hline$t$ & Time $[\mathrm{s}],[\mathrm{min}]$ \\
\hline$u$ & Velocity vector \\
\hline$V_{i}$ & Volume $\left[\mathrm{cm}^{3}\right]$ \\
\hline$W$ & Gain or loss of energy [Einstein $\mathrm{s}^{-1} \mathrm{~m}^{-3} \mathrm{sr}^{-1}$ ] \\
\hline$x$ & Position vector $[\mathrm{m}]$ \\
\hline$X_{i}$ & Independent variable \\
\hline$X_{j}$ & Independent variable \\
\hline$y$ & Dependant variable \\
\hline Y & Predicted response \\
\hline$Y_{1}$ & Percent TOC removal [\%] \\
\hline$Y_{2}$ & "Pseudo"-second order rate constant $\left[\mathrm{M}^{-1} \mathrm{~s}^{-1}\right]$ \\
\hline
\end{tabular}


$z \quad$ Optimal values of the kinetic parameter

$z_{L} \quad$ Length of the lamp [cm]

Greek Letters

$\begin{array}{ll}\beta_{0} & \text { Constant coefficient (intercept) } \\ \beta_{i} & \text { Linear coefficient } \\ \beta_{i i} & \text { Quadratic coefficient } \\ \beta_{i j} & \text { Interaction coefficient } \\ \xi_{i} & \text { Controllable input variables } \\ \Gamma & \text { Gamma function } \\ \Omega & \text { Kernal function, solid angle [sr] } \\ \delta & \text { Dirac delta function } \\ \varepsilon & \text { Molar extinction coefficient }\left[\mathrm{M}^{-1} \mathrm{~cm}^{-1}\right] \\ \theta & \text { Spherical coordinate [rad] } \\ \lambda & \text { Wavelength [nm] } \\ \mu & \text { Parameter defined in Equation }(2.26) \\ \rho & \text { Spherical coordinate [m] } \\ \sigma & \text { Volumetric scattering coefficient }\left[\mathrm{m}^{-1}\right] \\ \varphi & \left.\text { Quantum yield [mol Einstein }{ }^{-1}\right] \\ \phi & \text { Spherical coordinate [rad], vector containing } m \text { system input parameters }\end{array}$

Acronyms

2D Two dimensional

3D Three dimensional

ANOVA Analysis of Variance 
AOTs Advanced Oxidation Technologies

CCD Central Composite Design

df Degree of Freedom

ESVE Extense source model with voluminal emission

ESSE Extense source model with superficial emission

F-Value Fischer's Value

LCA Long Chain Approximation

LVREA Local Volumetric Rate of Energy Absorption

M Monomer

MS Mean square

MW Molecular weight

MWD Molecular weight distribution

ODEs Ordinary Differential Equations

p-Value Probability Value

PAA Poly(acrylic acid)

PVP Poly(vinyl pyrrolidone)

PBEs Population Balance Equations

PEG Poly(ethylene glycol)

PEO Poly(ethylene oxide)

RSM Response Surface Methodology

RTE Rate Transfer Equation

SS Sum of squares

TIC Theil's Inequality Coefficient

TOC Total Organic Carbon

UV Ultraviolet 


\section{Bibliography}

Aarthi, T.; Shaama, M.S.; Madras, G. Degradation of water soluble polymers under combined ultrasonic and ultraviolet radiation. Ind. Eng. Chem. Res., 46 (19), 6204-6210, 2007.

Adams, C.D.; Kuzhikannil, J.J. Effects of $\mathrm{UV} / \mathrm{H}_{2} \mathrm{O}_{2}$ preoxidation on the aerobic biodegradability of quaternary amine surfactants. Water Res. 34 (2), 668-672, 2000.

Alfano, O. M.; Romero, R.L.; Cassano, A.E. Radiation field modelling in photoreactors-I. homogeneous media. Chem. Eng. Sci. 41 (3), 421-444, 1986.

Alfano, O. M.; Cassano, A.E. Scaling-Up of Photoreactors. Applications to Advanced Oxidation Processes. Adv. Chem. Eng. 36, 229-287, 2009.

Alfassi, Z.B. The Chemistry of Free Radicals: Peroxyl Radicals. John Wiley \& Sons, New York, 1997.

Alpert, S. M.; Knappe, D.R.U.; Ducoste, J.J. Modeling the UV/hydrogen peroxide advanced oxidation process using computational fluid dynamics. Water Res. 44 (6), 1797-1808, 2010.

Ani, El. K.; Ramadhan, E. A. Photodegradation kinetics of poly(para-substituted styrene) in solution. Polym. Degrad. Stab., 93 (8), 1590-1596, 2008.

Audenaert, W. T. M.; Vermeersch, Y.; Van Hulle, S. W. H.; Dejans, P.; Dumoulin, A.; Nopens, I. Application of a mechanistic UV/hydrogen peroxide model at full-scale: Sensitivity analysis, calibration and performance evaluation. Chem. Eng. J. 17(1), 113-126, 2011. 
Ay, F.; Catalkaya, E.C.; Kargi, F. A statistical experiment design approach for advanced oxidation of Direct Red azo-dye by photo-Fenton treatment. J. Hazard. Mater.162 (1), 230$236,2009$.

Bach, A.; Shemer, H.; Semiat, R. Kinetics of phenol mineralization by Fenton-like oxidation. Desalination. 264 (3), 188-192, 2010.

Barrera, M.; Mehrvar, M., Gilbride, K. A.; McCarthy, L. H.; Laursen, A. E.; Boston, E.; Pushchak, R. Photolytic treatment of organic constituents and bacterial pathogens in secondary effluent of synthetic slaughterhouse wastewater. Chem. Eng. Res. Des. 90 (9), 1335-1350, 2012.

Bayraktar, E. Response surface optimization of the separation of DL-tryptophan using an emulsion liquid membrane. Process Biochem. 37 (2), 169-175, 2001.

Beltrán, F.J.; Aguinaco, A.; García-Araya, J. F. Kinetic modeling of TOC removal in the photocatalytic ozonation of diclofenac aqueous solutions. Appl. Catal., B. 100 (1-2), 289298, 2011.

Beltrán, F. J.; González M.; Rivas J.; Jaramillo J. Application of photochemical reactor models to UV radiation of trichloroethylene in water. Chemosphere. 31 (3), 2873-2885, 1995.

Bénard, A. C.; Darcos, V.; Drakides, C.; Casellas, C.; Coudane, J.; Vert, M. Fluorescence verses radioactivity labeling for laboratory-scale investigation of the fate of water-soluble polymers in wastewater treatment plants. J. Polym. Environ. 19 (1), 40-48, 2011.

Bielski, B. H. J.; Cabelli, D. E.; Arudi, R. L.; Ross, A. B. Reactivity of $\mathrm{HO}_{2} / \mathrm{O}_{2}{ }^{-}$radicals in aqueous solution. J. Phys. Chem. Ref. Data. 14 (4), 1041-1100, 1985. 
Bird, R. B.; Stewart, W. E.; Lightfoot, E. L. Transport Phenomena, second ed.; J. Wiley \& Sons: New York, Ch 18, 2002.

Box, G. E. P., and Behnken, D. W. Some New Three-Level Designs for the Study of Quantitative Variables. Technometrics, 2, 455-475, 1960.

Box, G. E. P.; Wilson, K. B. On the Experimental Attainment of Optimum Conditions. J. R. Stat .Soc. Series B. Stat. Methodol. 13 (1), 1-45, 1951.

Braun, A. M., Jakob, L., Oliveros, E. Advanced Oxidation Processes - Concepts of Reactor Design. J. Water SRT-Aqua. 42, 166-173, 1993.

Bruns, M.C.; Koo, J.H.; Ezekoye, O.A. Population-based models of thermoplastic degradation: Using optimization to determine model parameters. Polym. Degrad. Stab. 94 (6), 1013$1022,2009$.

Buxton G. V.; Greenstock C. L.; Helman W. P.; Ross A. B. Critical review of rate constants for reactions of hydrated electrons, hydrogen atoms and hydroxyl radicals $\left({ }^{\circ} \mathrm{OH} /{ }^{\circ} \mathrm{O}^{-}\right)$in aqueous solution. J. Phys. Chem. Ref. Data. 17 (2), 513-886, 1988.

Cao, W.; Mehrvar, M. Slaughterhouse wastewater treatment by combined anaerobic baffled reactor (ABR) and $\mathrm{UV} / \mathrm{H}_{2} \mathrm{O}_{2}$ processes. Chem. Eng. Res. Des. 89 (7), 1136-1143, 2011.

Carroccio, S.; Puglisi, C. Comparison of photooxidation and thermal oxidation processes in poly (ether imide). Macromol., 38 (16), 6863-6870, 2005.

Cassano, A.E. ; Martín, C.A.; Brandi, R.J.; Alfano, O.M. Photoreactor analysis and design: Fundamentals and applications. Ind. Eng. Chem. Res. 34 (7), 2155-2201, 1995. 
Cerdá, J., Marchetti, J. L. and Cassano, A. E. The use of radiation models for the case of direct irradiation of photochemical reactors. Lat. Am. J. Chem. Engng appl. Chew. 8, 15-25, 1978.

Chen, R.; Pignatello, J. J. Role of quinone intermediates as electron shuttles in fenton and photoassisted fenton oxidations of aromatic compounds. Environ. Sci. Technol. 31, 23992406, 1997.

Christensen, H.; Sehested, K.; Corfitzen, H. Reactions of hydroxyl radicals with hydrogen peroxide at ambient and elevated temperatures. J. Phys. Chem. 86, 1588-1590, 1982.

Crittenden, J. C.; Hu, S.; Hand, D. W.; Green, S. A. A kinetic model for $\mathrm{H}_{2} \mathrm{O}_{2} / \mathrm{UV}$ process in a completely mixed batch reactor. Water Res. 33(10), 2315-2328, 1999.

Daraboina, N.; Madras, G. Kinetics of ultrasonic degradation of poly (alkyl methacrylates). Ultrason. Sonochem. 16, 273-279, 2009.

De Laat, J.; Le, T.G. Effects of chloride ions on the iron(III)-catalyzed decomposition of hydrogen peroxide and on the efficiency of the Fenton-like oxidation process. Appl. Catal., B. 66, 137-146, 2006.

Dopar, M., Kusic, H., Koprivanac, N. Treatment of simulated industrial wastewater by photoFenton process. Part I: The optimization of process parameters using design of experiments (DOE). Chem. Eng. J. 173, 267-279, 2010.

Devi, L. G.; Rajashekhar, K.E.; Raju, K.S.A.; Kumar, S. G. Kinetic modeling based on the nonlinear regression analysis for the degradation of Alizarin Red S by advanced photo Fenton 
process using zero valent metallic iron as the catalyst. J. Mol. Catal. A: Chem. 314, 88-94, 2009.

Duran, J. E.; Mohseni, M.; Taghipour, F. Computational fluid dynamics modeling of immobilized photocatalytic reactors for water treatment. AIChE J. 57, 1860-1872, 2011.

Edalatmanesh, M.; Dhib, R.; Mehrvar, M. Kinetic modeling of aqueous phenol degradation by $\mathrm{UV} / \mathrm{H}_{2} \mathrm{O}_{2}$ process. Int. J. Chem. Kinet. 40, 34-43, $2008 \mathrm{~b}$.

Einschlag, G. F.; Féliz, M.R.; Capparelli, A.L. Effect of temperature on hydrogen peroxide photolysis in aqueous solutions. J. Photochem. Photobiol. A. 110, 235-242, 1997.

Elliot, A.J.; Buxton, G.V. Temperature dependence of the reactions $\mathrm{OH}+\mathrm{O}_{2}^{-}$and $\mathrm{OH}+\mathrm{HO}_{2}$ in water up to $200^{\circ}$ C. J. Chem. Soc., Faraday Trans. 88, 2465-2470, 1992.

Farias, J.; Albizatti, E.D.; Alfano, O.M. Kinetic study of the photo-Fenton degradation of formic acid Combined effects of temperature and iron concentration. Catal. Today. 1-2, 117-123, 2009.

Fenton H. J. H. Oxidation of tartaric acid in presence of iron. J. Chem. Soc. 65,899-910, 1894.

Fernández, M.D.; Fernández, M.J. Thermal degradation of copolymers from vinyl acetate and vinyl alcohol. J. Therm. Anal. Calorim. 92, 829-837, 2008.

Fernando, S.S.; A. Christensen, P.; A. Egerton, T; R.White, J. Carbon dioxide evolution and carbonyl group development during photodegradation of polyethylene and proproylene. Polym. Degrad. Stab., 92 (12), 2163-2172, 2007.

Gavalas, G.R. The long chain approximation in free radical reaction systems. Chem. Eng. Sci. 21 (2), 133-142, 1966. 
Ghafoori, S., Mehrvar, M., Chan, P. Photoassisted Fenton-like degradation of aqueous poly(acrylic acid): From mechanistic kinetic model to CFD modeling. Chem. Eng. Res. Des. 2013a (in press).

Ghafoori, S., Mehrvar, M., Chan, P. Optimization of photo-Fenton-like degradation of aqueous polyacrylic acid using Box-Behnken experimental design. Can. J. Chem. Eng. 2013b, Published online.

Ghafoori, S.; Mehrvar, M.; Chan, P. Kinetic study of photodegradation of water soluble polymers. Iran. Polym. J. 21:869-876, 2012a.

Ghafoori, S.; Mehrvar, M.; Chan, P. Free-radical-induced degradation of aqueous Polyethylene Oxide by $\mathrm{UV} / \mathrm{H}_{2} \mathrm{O}_{2}$ : Experimental design, reaction mechanisms, and kinetic modeling. Ind. Eng. Chem. Res. 51, 14980-14993, 2012 b.

Giménez, J.; Curcó, D. Photocatalytic treatment of phenol and 2,4-dichlorophenol in a solar plant in the way to scaling-up. Catal. Today. 54. 229-243, 1999.

Giroto, J.A.; Teixeira, A.C.S.C.; Nascimento, C.A.O.; Guardani, R. Degradation of Poly(ethylene glycol) in aqueous solution by photo-Fenton and $\mathrm{H}_{2} \mathrm{O}_{2} / \mathrm{UV}$ processes. Ind . Eng. Chem. Res. 49, 3200-3206, 2010.

Giroto, J.A.; Teixeira, A.C.S.C.; Nascimento, C.A.O; Guardani, R. Photo-fenton removal of water-soluble polymers. Chem. Eng. Process., 47 (12), 2361-2369, 2008.

Grcic, I, M. Maljkovic, S. Papic, Koprivanac, N. Low frequency US and UV-A assisted Fenton oxidation of simulated dyehouse wastewater. J. Hazard. Mater. 197, 279-284, 2011. 
Guang-meng, R.; De-zhi, S.;Chuank, J.S. Advanced treatment of oil recovery wastewater from polymer flooding by $\mathrm{UV} / \mathrm{H}_{2} \mathrm{O}_{2} / \mathrm{O}_{3}$ and fine filtration. J. Environ. Sci., 18(1), 29-32, 2006.

Haber F, Weiss J. The catalytic decomposition of hydrogen peroxide by iron salts. Proc. $R$ Soc. Ser. A. Math. Phys. Sci.147, 332-351, 1934.

Hassouna, F.; Mailhot, G.; Morlat-Thérias, S.; Gardette, J.L. Influence of iron salts on the photooxidation of poly(N-vinylpyrrolidone) in aqueous solution. J. Photochem. Photobiol. A. 218, 239-246, 2011.

Hassouna, F.; Morlat-Therias, S.; Mailhot, G.; Gardette, J. Photooxidation of poly(Nvinylpyrrolidone) (PVP) in the solid state in aqueous solution. Polym. Degrad. Stab. 94, 2257-2266, 2009.

Hassouna, F.; Morlat-Therias, S.; Mailhot, G.; Gardette, J. Influence of water on the photodegradation of poly(ethylene oxide). Polym. Degrad. Stab. 92 (11), 2042-2050, 2007.

Herney-Ramireza, J.; Vicente, M. A.; Madeira, L. M. Heterogeneous photo-Fenton oxidation with pillared clay-based catalysts for wastewater treatment: A review. Appl. Catal., B. 98, 10-26, 2010.

Hsu, L. J.; Lee, L. T.; Lin, C. C. Adsorption and photocatalytic degradation of polyvinyl alcohol in aqueous solutions using $\mathrm{P}-25 \mathrm{TiO}_{2}$. Chem. Eng. J. 173, 698-705, 2011.

Iordan, A.R.; Palamaru, M.; Cecal, A. ; Popa, A.F. Photochemical degradation of polymers containing urethane groups in the solid phase: Influence of $\mathrm{S}$ block cations on the degradation. Int. J. Polym. Mater., 53 (2), 157-172, 2004. 
Irazoqui. H. A.; Cerdá, J.; Cassano, A. E. Radiation profiles in an empty annular photoreactor with a source of finite spatial dimensions. AIChE. J. 19, 460-467, 1973.

Jacob, S. M.; Dranoff, J. S. Radial scale-up of perfectly mixed photochemical reactors. Chem. Engng Prog. Symp. Sm. 62, 47-55, 1966.

Johnson, M. B.; Mehrvar, M. Aqueous metronidazole degradation by $\mathrm{UV} / \mathrm{H}_{2} \mathrm{O}_{2}$ process in single-and multi-lamp tubular photoreactors: Kinetics and reactor design. Ind. Eng. Chem. Res. 47, 6525, 2008.

Jung, Y.S., Lim, W.T., Park, J.Y., Kim, Y.H. Effect of pH on Fenton and Fenton-like oxidation. Environ. Technol. 30, 183-190, 2009.

Kaczmarek, H.; Kaminska, A.; Swiatek, M.; Rabek, J. F. Photo-oxidative degradation of some water-soluble polymers in the presence of accelerating agents. Angew. Makromol. Chem. 4622, 109-121, 1998.

Kaczmarek, H.; Kowalonek, J.; Klusek, Z.; Pierzgalski, S.; Datta, S. Studies of photooxidative degradation of poly(vinyl chloride)/poly(ethylene oxide)blends. J. Polym. Sci., Part B: Polym. Phys. 42 (4), 585-602, 2004.

Kanwal, F., Pethrick, R. A. Ultrasonic degradation studies of poly(ethylene oxide), poly(ethylene adipate) and poly(dimethylsiloxane). Polym. Degrad. Stab.84, 1-6, 2004.

Kodera, Y.; J. McCoy, B. Distribution kinetics of radical mechanisms: reversible polymer decomposition. AIChE J., 43 (12), 3205-3214, 1997.

Konaganti, V. K.; Madras, G. Photooxidative and pyrolytic degradation of methyl methacrylatealkyl acrylate copolymers. Polym. Degrad. Stab. 94, 1325-1335, 2009. 
Konaganti, V.K.; Madras, G. Ultrasonic degradation of poly(methyl methacrylate-co-alkyl acrylate) copolymers. Ultrasonics Sonochemistry., 17 (2), 403-408, 2010.

Kossiakoff, A., Rice, F.O. Thermal decomposition of hydrocarbons, resonance stabilization and isomerization of free radicals. J. Am. Chem. Soc., 65 (4), 590-595, 1943.

Koppenol, W. H.; Butler, J.; Van Leeuwen, J. W. The Haber-Weiss cycle. Photochem. Photobiol. 28, 655-658, 1978.

Kralik, P., Kusic, H., Koprivanac, N., Loncaric Bozic, A. Degradation of chlorinated hydrocarbons by $\mathrm{UV} / \mathrm{H}_{2} \mathrm{O}_{2}$ : The application of experimental design and kinetic modeling approach. Chem, Eng. J. 158 (2), 154-166, 2010.

Kusic, H.; Koprivanac, N.; Horvat, S.; Bakija, S.; Bozic, A. L. Modeling dye degradation kinetic using dark- and photo-Fenton type processes. Chem. Eng. J. 155, 144-154, 2009.

Labas, M. D.; Zalazar, C. S.; Brandi, R. J.; Martín, C.A.; Cassano, A. E. Scaling up of a photoreactor for Formic Acid degradation employing hydrogen peroxide and UV radiation. Helv. Chim. Acta. 85, 82-95, 2002.

Ledakowicz, S.; Solecka, M. Impact of advanced oxidation processes on the biodegradation kinetics of industrial wastewater. Water Sci. Technol. 41, 157-164, 2000.

Lépine, L.; Gilbert, R. Thermal degradation of polyacrylic acid in dilute aqueous solution. Polym. Degrad. Stab. 75, 337-345, 2002.

Levenspiel O. Chemical Reaction Engineering, 2nd ed., John Wiley \& Sons, New York, 1972. 
Linden, K. G.; Sharpless, C. M.; Andrews, S.; Atasi, K.; Korategere, V.; Stefan, M.; Suffet, I. H. M. Innovative UV Technologies to Oxidize Organic and Organoleptic Chemicals; IWA Publishing: London, Ch 8, 2005.

Lu, M.; Wu, X.; Wei, X. Chemical degradation of polyacrylamide by advanced oxidation processes. Environ. Technol. 33, 1021-1028, 2012.

Madras, G; Chung, G. Y; Smith J. M; McCoy B. J. Molecular weight effect on the dynamics of polystyrene degradation. Ind. Eng. Chem. Res. 36, 2019-2024, 1997.

Madras, G.; Smith, J.M.; McCoy, B.J. Effect of tetralin on the degradation of polymer in solution. Ind. Eng. Chem. Res., 34 (12), 4222-4228, 1995.

Martins-Franchetti, S.M.; Campos, A.; Egerton, T.; White, R. J. Structural and morphological changes in poly(caprolactone)/poly(vinyl chloride) blends caused by UV irradiation. $J$. Mater. Sci., 43 (3), 1063-1069, 2008.

Marugan, J.; Grieken, R. V.; Cassano, A. E.; Alfano, O. M. Scaling-up of slurry reactors for the photocatalytic oxidation of cyanide with $\mathrm{TiO}_{2}$ and silica-supported $\mathrm{TiO}_{2}$ suspensions. Catal. Today. 144, 87-93, 2009.

McCoy, B.; Madras, G. Degradation Kinetics of Polymers in Solution: Dynamics of Molecular Weight Distributions. AIChE J., 43 (3), 802-810, 1997.

McCoy, B. J.; Wang, M. Continuous-mixture fragmentation kinetics: particle size reduction and molecular cracking. Chem. Eng. Sci. 49, 3773, 1994. 
Mehrvar, M., Anderson, W.A., Moo-Young, M., Reilly, P.M. Non-linear parameter estimation for a dynamic model in photocatalytic reaction engineering. Chem. Eng. Sci. 55 (21), 48854891, 2000.

Mehrvar, M.; Anderson, W.A.; Moo-Young, M. Photocatalytic degradation of aqueous organic solvents in the presence of hydroxyl radical scavengers. Int. J. Photoenergy. 3 (4). 187-191, 2001.

Meulemans C. C. The Basic Principles of UV Disinfection in Water. Ozone. Sci. Eng . 9, 299$313,1987$.

Mohajerani, M.; Mehrvar, M.; Ein-Mozaffari, F. An overview of the integration of advanced oxidation technologies and other processes for water and wastewater treatment. Int. J. Eng. 3, 120-146, 2009.

Mohajerani, M.; Mehrvar, M.; Ein-Mozaffari, F. CFD modelling of metronidazole degradation in water by $\mathrm{UV} / \mathrm{H}_{2} \mathrm{O}_{2}$ process in single and multi-lamp photoreactors. Ind. Eng. Chem. Res. 49, 5347, 2010.

Mohajerani, M.; Mehrvar, M.; Ein-Mozaffari, F. Photoreactor design and CFD modeling of a $\mathrm{UV} / \mathrm{H}_{2} \mathrm{O}_{2}$ process for distillery wastewater treatment. Can. J. Chem. Eng. 90, 719-729, 2012a.

Mohajerani, M.; Mehrvar, M.; Ein-Mozaffari, F. Using an external-loop airlift sonophotoreactor to enhance the biodegradability of aqueous sulfadiazine solution. Sep. Purif. Technol. 90, 173-181, 2012b. 
Myers, R.H.; Mongomery, D.C.; Anderson-Cook, C. M. Process and Product Optimization using Designed Experiments. 3rd edition, John Wiley \& Sons, Inc., Hoboken, New Jersey, 2009.

Nigam, A.; Fake, D.M.; Klein, M.T. Simple approximate rate law for both short- and long-chain Rice Herzfeld kinetics. AIChE J. 40, 908-910, 1994.

Oppenländer, T. Photochemical Purification of Water and Air: Advanced Oxidation Processes, Wiley-VCH., Weinheim, 2003.

Pérez-Moya, M.; Graells, M.; Buenestado, P.; Mansilla, H.D. A comparative study on the empirical modeling of photo-Fenton treatment process performance. Applied Catalysis B: Environmental. 84, 313-323, 2008.

Perry, R. H.; Green, D. W.; Maloney, J. D. Perry's Chemical Engineer's Handbook; McGrawHill: New York, Ch 7, 1981.

Pignatello J. J.; Liu D.; Huston P. Evidence for an additional oxidant in the photoassisted Fenton reaction. Environ. Sci. Technol. 33, 1832-1839, 1999.

Pontes, R. F. F.; Moraes, J. E.F.; Machulek, A. M. Jr., Pinto, J. M. A mechanistic kinetic model for phenol degradation by the Fenton process. J. Hazard. Mater. 176, 402-413, 2010.

Poyatos, J.M.; Monio, M.M.; Almecija, M.C.; Torres, J.C.; Hontoria, E.; Osorio, F. Advanced oxidation processes for wastewater treatment: state of the art. Water Air Soil Pollut. 205, 187-204, 2010.

Pulgarin, C.; Kiwi, J. Overview on photocatalytic and electrocatalytic pretreatment of industrial non-biodegradable pollutants and pesticides. Chimia. 50, 50-55, 1996. 
Ranby, B. Photodegradation and photo-oxidation of synthetic polymers. J. Anal. Appl. Pyrolysis., 15, 237-247, 1989.

Ranby, B.; Rabek, J.F. Photodegradation, Photo-oxidation and Photostabilization of Polymers. John Wiley and Sons, London, 1975.

Reich, L.; Stivala, S. Elements of Polymer Degradation. McGraw Hill, New York, 1971.

Rice, F. O.; Herzfeld, K. F. The mechanism of some chain reactions. J. Chem. Phys. 7, 671, 1939.

Rodríguez, M.; Malato, S.; Pulgarin, C.; Contreras, S.; Curco, D.; Gimenez, J.; Esplugas, S. Optimizing the solar photo-Fenton process in the treatment of contaminated water. Determination of intrinsic kinetic constants for scale-up. Sol. Energy. 79, 360-368, 2005.

Rojas, M. R.; Pérez, F.; Whitley, D.; Arnold, R. G.; Sáez, A. E. Modeling of Advanced Oxidation of Trace Organic Contaminants by Hydrogen Peroxide Photolysis and Fenton's Reaction. Ind. Eng. Chem. Res. 49, 11331-11343, 2010.

Roy, P.K.; Surekha, P.; Raman, R.; Rajagopal, C. Investigating the role of metal oxidation state on the degradation behaviour of LDPE. Polym. Degrad. Stab., 94 (7), 1033-1039, 2009.

Santro, D.; Raisee, M.; Moghaddami, M.; Ducost, J.; Sasges, M.; Liberti, L.; Notarnicola, M. Modeling hydroxyl radical distribution and trialkyl phosphates oxidation in $\mathrm{UV}-\mathrm{H}_{2} \mathrm{O}_{2}$ photoreactors using computational fluid dynamics. Environ. Sci. Technol. 44, 6233-6241, 2010.

Santos L.C., Poli A.L., Cavalheiro C.C.S., Neumann M.G. The UV/ $\mathrm{H}_{2} \mathrm{O}_{2}$ photodegradation of poly(ethylene glycol) and model compounds. J. Braz. Chem. Soc. 20, 1467-1472, 2009. 
Santos, K.A.M.; Suarez, P.A.Z.; Rubim, J.C. Photodegradation of synthetic and natural polyisoprenes at specific UV radiations. Polym. Degrad. Stab., 90 (1), 34-43, 2005.

Satuf, A. L.; Brandi, R. J.; Cassano, A. E.; Alfano, O. M. Scaling-up of slurry reactors for the photocatalytic degradation of 4-chlorophenol. Catal. Today. 129, 110-117, 2007.

Sezgi, A. N.; S. Cha, W.; Smith, J. M.; J. McCoy, B. Polyethylene pyrolysis: Theory and experiments for molecular-weight-distribution kinetics. Ind. Eng. Chem. Res., 37 (7), 2582$2591,1998$.

Sharpless, C. M., Linden, K. G. Experimental and model comparisons of low- and mediumpressure $\mathrm{Hg}$ lamps for the direct and $\mathrm{H}_{2} \mathrm{O} 2$ assisted $\mathrm{UV}$ photodegradation of $\mathrm{N}$ nitrosodimethylamine in simulated drinking water. Environ. Sci. Technol. 37 (9), 1933$1940,2003$.

Shukla, B.N.; Daraboina, N.; Madras, G. Oxidative and photooxidative degradation of poly (acrylic acid). Polym. Degrad. Stab., 94 (8), 1238-1244, 2009.

Silva, T. C. F.; Silva, C. M.; Reis, C.; Bellato, C. R.; Lucia, L. A. Application of Photo-oxidative Processes for the Remediation of Bleached Kraft Pulp Effluent. Ind. Eng. Chem. Res. 49, 11214-11220, 2010.

Simha, R.; Wall, L. A. Mechanisms of Polper Formation and Decomposition. Catalysis; RienMd: New York, 1985.

Simmler, W. Water, in: Ullmann's Encyclopedia of Industrial Chemistry. $5^{\text {th }}$ ed., Vol. B 8:1152, VCH, Weinheim, 1995. 
Simunovic, M.; Kusic, H.; Koprivanac, N.; Bozic, A. L. Treatment of simulated industrial wastewater by photo-Fenton process: Part II. The development of mechanistic model. Chem. Eng. J. 173, 280- 289, 2011.

Smagala T.G.; McCoy, B.J. Mechanisms and approximations in macromolecular reactions: reversible initiation, chain scission, and hydrogen abstraction. Ind. Eng. Chem. Res., 42 (12), 2461-2469, 2003.

Sonntag C.; Schuchmann H.P. The Elucidation of Peroxyl Radical Reactionsmin Aqueous Solution with the Helpmof Radiation-Chemical Methods. Angew. Chem. Int. Ed. Engl. 30, $1229-1253,1991$.

Sterling, W. J.; McCoy, B. J. Distribution kinetics of thermolytic macromolecular reactions. AlChE J. 47, 2289-2303, 2001.

Stramigioli, C.; Santarelli, F.; Foraboschi, F. P. Photosensitized reactions in an annular continuous photoreactor. Appl. Sci. Res. 33, 2342, 1977.

Suji, T.H.; Echizen, Y.; Nishimura, Y. Photodegrdation of biodegradable polyesters: a comprehensive study on poly(L.lactic) and poly(e-caprolactone). Polym. Degrad. Stab., 91 (5), 1128-1137, 2006.

Sun, S. P.; Lemley, A. T. p-Nitrophenol degradation by a heterogeneous Fenton-like reaction on nano-magnetite: Process optimization, kinetics, and degradation pathways. J. Mol. Catal. A: Chem. 349, 71-79, 2011.

Swift, G. Directions for environmentally bioderadable polymer research. Acc. Chem. Res. 26, 105-110, 1993. 
Tabrizi, G.B.; Mehrvar, M. Integration of advanced oxidation technologies and biological processes: Recent developments, trends, and advances. J. Environ. Sci. Health., Part A. 39, 3029-3081, 2004.

Tabrizi, G. B.; Mehrvar, M. Pilot-plant study for the photochemical treatment of aqueous linear alkylbenzene sulfonatz. Sep. Purif. Technol. 49 (2), 115-121, 2006.

Tanyildizi, M.S. Modeling of adsorption isotherms and kinetics of reactive dye from aqueous solution by peanut hull. Chem. Eng. J. 168, 1234-1240, 2011.

Tarr M.A. Ozone-UV radiation-hydrogen peroxide oxidation technologies. In: Chemical Degradation Methods for Wastes and Pollutants. Marcel Dekker Inc., New York, 2003.

Trujillo, F. J.; Safinski, T.; Adesina, A. A. Oxidative photomineralization of dichloroacetic Acid in an externally-irradiated rectangular bubble tank reactor: computational fluid dynamics modeling and experimental verification studies. Ind. Eng. Chem. Res. 49, 6722-6734, 2010 .

Varma, A., Morbidelli, M., Wu, H. Parametric Sensitivity in Chemical Systems. Cambridge University Press, Cambridge, 1999.

Vijayalakshmi, S.P.; Raichur, A.; Madras, G. Thermal degradation of poly(ethylene oxide) and polyacrylamide with ascorbic acid. J. Appl. Polym. Sci., 101, 3067-3072, 2006.

Vincent, G.; Schaer, E.; Marquaire, P.; Zahraa, O. CFD modelling of an annular reactor, application to the photocatalytic degradation of acetone. Process Saf. Environ. 89, 35-40, 2011. 
Vrandečić, N.S.; Erceg, M.; Jakić, M.; Klarić, I. Kinetic analysis of thermal degradation of poly(ethylene glycol) and poly(ethylene oxide)s of different molecular weight. Thermochim. Acta. 498, 71-80, 20101.

Wang, M.; Smith, J.M.; McCoy, B. J. Continuous kinetics for thermal degradation of polymer in solution. AIChE J., 41 (6), 1521-1533, 1995.

Weinstein, J.; Bielski, B. H. J. Kinetics of the interaction of $\mathrm{HO}_{2}$ and $\mathrm{O}_{2}^{-}$radicals with hydrogen peroxide: The Haber-Weiss reaction. J. Am. Chem. Soc.101, 58-62, 1979.

White, J.R.; Shyichuk, A.V. Macromolecular scission and crosslinking rate changes during polyolefin photo-oxidation. Polym. Degrad. Stab. 92 (7), 1161-1168, 2007.

Williams, P.A. Handbook of Industrial Water Soluble Polymers; Blackwell-Ames: Iowa, Ch 1, 2007.

Wols, B.A., Hofman-Caris, C.H.M. Review of photochemical reaction constants of organic micropollutants required for UV advanced oxidation processes in water. Water Res. 46, 2815-2827, 2012.

Xu, B.; Gao, N.-Y.; Cheng, H.; Xia, S.-J.; Rui, M.; Zhao, D.-d. Oxidative degradation of dimethyl phthalate (DMP) by UV/ $\mathrm{H}_{2} \mathrm{O}_{2}$ process. J. Hazard. Mater. 162, 954-959, 2009.

Zhang, H.; Ran, X.; Wu, X., Zhang, D. Evaluation of electro-oxidation of biologically treated landfill leachate using response surface methodology. J. Hazard. Mater. 188, 261-268, 2011.

Ziff, R. M.; McGrady, E. D. Kinetics of polymer degradation. Macromol. 19, 2513-2519, 1986. 


\section{Appendices}

\section{Appendix A: TOC Calibration Curve}

The TOC was measured by a Tekmar Dohrmann's Apollo 9000 TOC analyzer. The analyzer uses combustion $\left(680\right.$ to $\left.1000^{\circ} \mathrm{C}\right)$ with a patented reusable platinum catalyst for the lowest detection limit while maximizing TOC recovery. The Non-Dispersive Infra-Red (NDIR) detector in the Apollo 9000 TOC Analyzer is sensitive for very low levels of TOC (4 $m g C / L)$ which directly and specifically measures the carbon dioxide generated by the oxidation of the organic carbon in the sample. Any potential interference is removed by inline scrubbers or filters as the sample gas is swept to the detector. The analyzer is able to measure TOC and TN simultaneously for the same sample with an optional module. It can measure TOC between 4-25,000 $m g \mathrm{C} / L$ range and $\mathrm{TN}$ from $0-200 m g \mathrm{~N} / L$ range. Approximately $15-40 \mathrm{~mL}$ sample was filled in a sampling vial and was placed in the auto sampler. Through running TOC Talk software (version 3.5), the TOC standard calibration

analysis was carried out using the working standard solutions. The TOC (Figures A.1 and A.2) calibration curve for the range of $1-4,000 \mathrm{mgC} / \mathrm{L}$ was obtained for analyzing TOC concentrations. Measurement of each sample was repeated in triplicate and an average value was reported as the TOC reading. A response factor of the instrument correlates the raw counts to a known amount of organic carbon in the standard. The calibration of the TOC was done once during the 9 month experimental study period.

The steps during the TOC analysis (Apollo 9000 TOC Analyzer Operation Manual, 2009) are described as follows: 
1. Sampling: Samples were injected into the analyzer with the help of an automated syringe from the sampling bottle. The sample injection valve automatically selects the appropriate sample volume for the optimum measuring range.

2. Inorganic Carbon (IC) detection: $20 \%$ phosphoric acid was added to lower the $\mathrm{pH}$ so that inorganic carbon was sparged off as $\mathrm{CO}_{2}$. This was measured to get IC content and to ensure that it was not carried over into the TOC. 


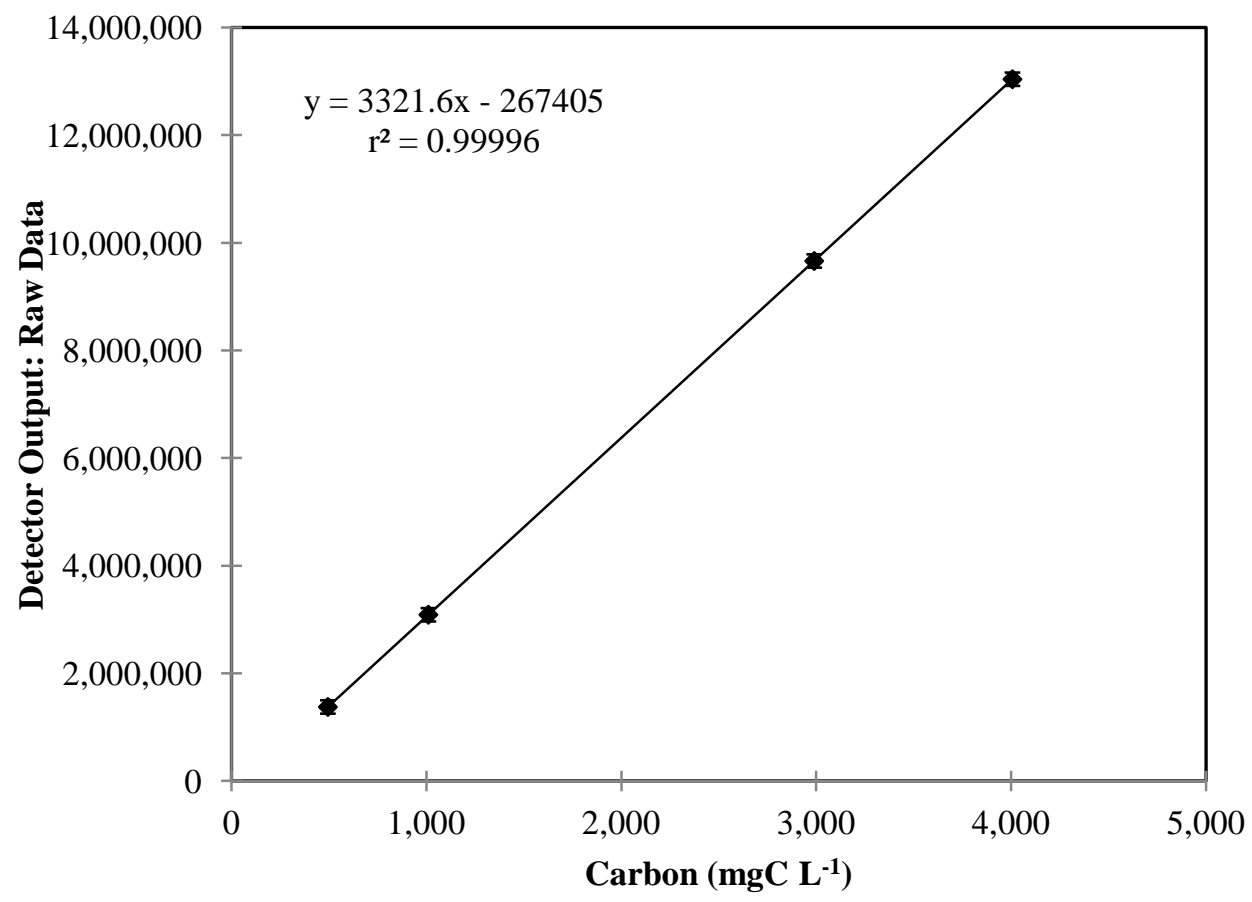

Figure A.1. TOC calibration curve plotted for output raw data from the detector versus the amount of organic carbon present, for the range of $1-4,000 \mathrm{mg} \mathrm{C} \mathrm{L}^{-1}$. 


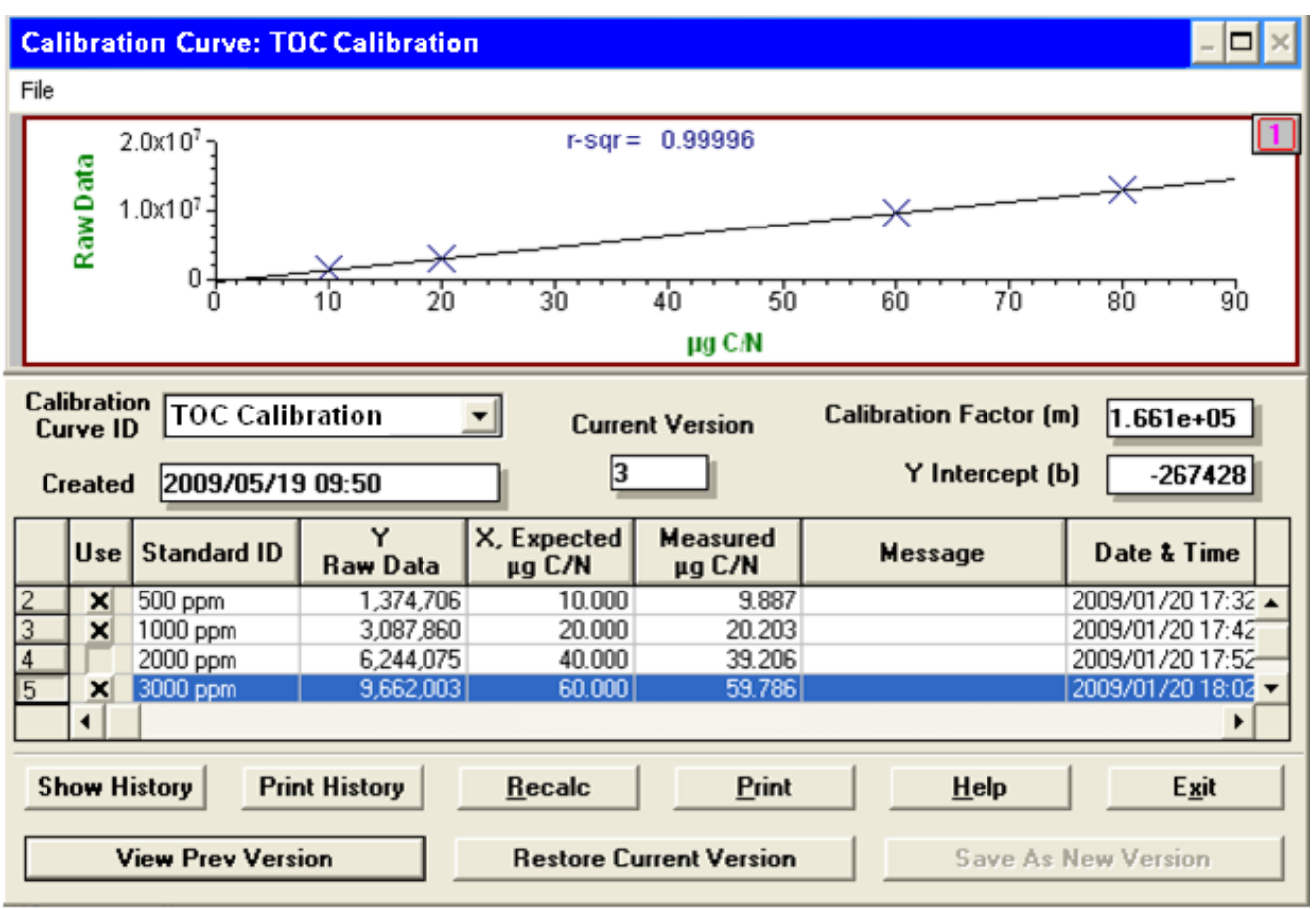

Figure A.2. TOC calibration curve for the range 0 - $60 \mu g \mathrm{C}$ from Apollo $9000 \mathrm{TOC} / \mathrm{TN}$ analyzer. 


\section{Appendix B: Computer Program}

A computer program is written in MATLAB to determine the model predictions for photodegradation of PEG using $\mathrm{UV} / \mathrm{H}_{2} \mathrm{O}_{2}$ process as presented in Section 4.1.

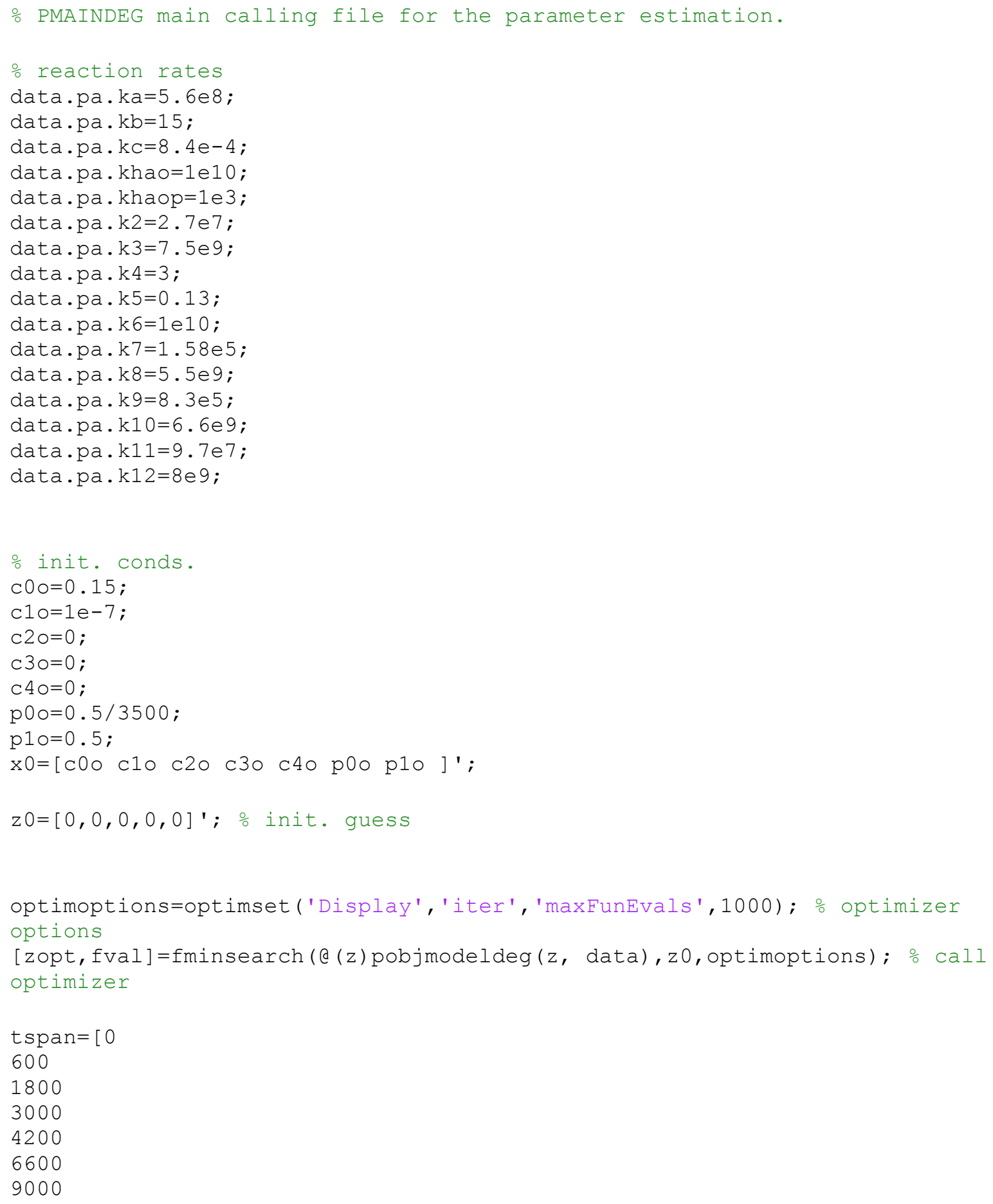


10200

11400

12600

]; o time for which you have data

$\mathrm{Mn}=[$

3500

1750

510

250

184

175

146

130

130

121

] ; $\frac{\circ}{0}$ Mn data points

options = odeset('RelTol',1e-4,'AbsTol', [1e-12 1e-12 1e-12 1e-12 1e-12 1e-12 $1 \mathrm{e}-12])$;

$[t, y]=0 d e 15 s\left(@(t, x)\right.$ pmodeldeg $(t, x, z o p t$, data $), t$ span, $x 0$, options ); $\frac{\circ}{\circ}$ integrate

$[B X, S 1, S 2]=p l o t y y\left(t / 60, y(:, 1), t / 60, y(:, 6)\right.$, plot $\left.^{\prime}\right) ;$

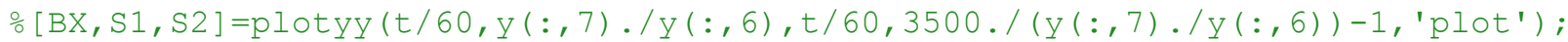
set (S1,'linestyle','-'); set(S2,'linestyle','--');

hold on;

oplot (t/60,3500./(y $\left.\left.(:, 5) \cdot / \mathrm{y}(:, 4)), \mathbf{1}^{\prime}-\right)^{\prime}\right)$;

oplot (tspan/60, (3500./Mn)-1,'or');

ohold on;

oplot $\left(t / 60,3500 . /(y(:, 5) . / y(:, 4)),{ }^{\prime}-'\right)$;

$\frac{0}{0}[\mathrm{AX}, \mathrm{H} 1, \mathrm{H} 2]=\operatorname{plotyy}(\operatorname{tspan} / 60, \mathrm{Mn}, \operatorname{tspan} / 60,(3500 . / \mathrm{Mn})-1)$;

oset (H1, 'linestyle', 'none', 'marker', 'o');

set (H2,' 'linestyle', 'none', 'marker', '^') ;

olegend([S1,S2,H1,H2], 'Predicted model for number average molecular weight','Predicted model for number of chain scission per

molecule','Experimental data', 'Calculated from experimental data');

legend([S1,S2],'Predicted model for H_2O_2 concentration','Predicted model

for total molar concentration of polymer');

oplot (tspan, y $\left.(:, 1),{ }^{-}-1\right)$;

oplot (tspan, y $(:, 4))$; 
A computer program is written in MATLAB to determine the model predictions for photodegradation of $\mathrm{PEO}$ using $\mathrm{UV} / \mathrm{H}_{2} \mathrm{O}_{2}$ process as presented in Section 4.2.

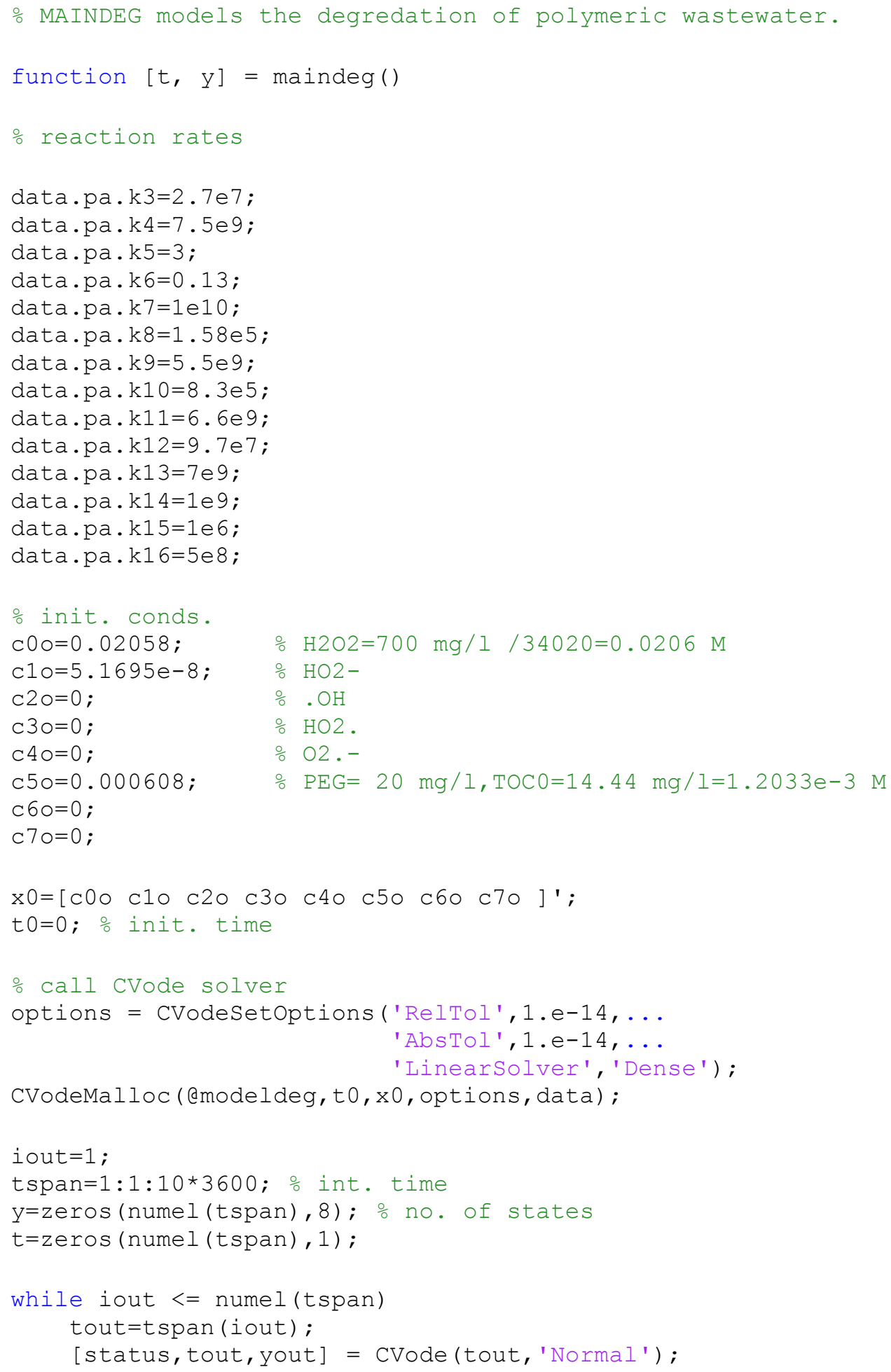




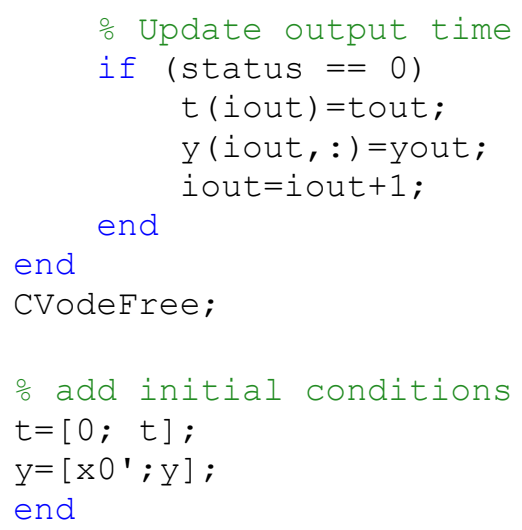


\% MODELDEG dynamic model for degradation polymeric wastewater.

function $[d x, f l a g$, new_data $]=\operatorname{modeldeg}(t, x$, data $)$

ㄴ. trans. vars.

$\mathrm{C} 0=\mathrm{x}(1) ;$

$\mathrm{C} 1=\mathrm{x}(2) ;$

$\mathrm{c} 2=\mathrm{x}(3) ;$

$\mathrm{c} 3=\mathrm{x}(4) ;$

$\mathrm{C} 4=\mathrm{x}(5) ;$

$\mathrm{c} 5=\mathrm{x}(6)$;

$\mathrm{c} 6=\mathrm{x}(7) ;$

$\mathrm{c} 7=\mathrm{x}(8) ;$

o get params.

data.pa.k3=2.7e7;

data.pa.k4=7.5e9;

data.pa.k5=3;

data.pa.k6=0.13;

data.pa.k7=1e10;

data.pa.k8=1.58e5;

data.pa.k9=5.5e9;

data.pa.k10=8.3e5;

data.pa.k11=6.6e9;

data.pa.k12=9.7e7;

data.pa.k13=7e9;

data.pa.k14=1e9;

data.pa.k15=1e6;

data.pa.k16=5e8;

\% model diff. eqs.

$\mathrm{dc} 0=0.092 *(-0.5 * 1.97 e-5 *((1870 * \mathrm{c} 0+21000 * \mathrm{c} 1) /(1870 * \mathrm{c} 0+21000 * \mathrm{c} 1+21396 * \mathrm{c} 5)) *(1-$ $\left.\exp \left(-2.303 * 0.00133 *\left(1870 * \mathrm{c} 0+21000 * \mathrm{c} 1+21396{ }^{*} \mathrm{c} 5\right)\right)\right)-\mathrm{k} 3{ }^{*} \mathrm{c} 0{ }^{*} \mathrm{c} 2-\mathrm{k} 4{ }^{*} \mathrm{c} 1{ }^{*} \mathrm{c} 2-\mathrm{k} 5{ }^{*} \mathrm{c} 0{ }^{*} \mathrm{c} 3-$ $\mathrm{k} 6 * \mathrm{c} 0 * \mathrm{c} 4+\mathrm{k} 9 * \mathrm{c} 2 * \mathrm{c} 2+\mathrm{k} 10 * \mathrm{c} 3 * \mathrm{c} 3+\mathrm{k} 12 * \mathrm{c} 3 * \mathrm{c} 4) *(1 /(1+10 e-6)) ;$

$\mathrm{dc} 1=0.092 *\left(-0.5 * 1.97 e-5 *\left((1870 * \mathrm{c} 0+21000 * \mathrm{c} 1) /\left(1870 *_{\mathrm{c}} 0+21000 * \mathrm{c} 1+21396 * \mathrm{c} 5\right)\right) *(1-\right.$ $\exp (-2.303 * 0.00133 *(1870 * c 0+21000 * c 1+21396 * c 5)))-k 3 * c 0 * c 2-k 4 * c 1 * c 2-k 5 * c 0 * c 3-$ $\left.\mathrm{k} 6{ }^{*} \mathrm{c} 0 * \mathrm{c} 4+\mathrm{k} 9{ }^{*} \mathrm{c} 2{ }^{*} \mathrm{c} 2+\mathrm{k} 10 * \mathrm{c} 3{ }^{*} \mathrm{c} 3+\mathrm{k} 12{ }^{*} \mathrm{c} 3 * \mathrm{c} 4\right) *(1 /(1+10 \mathrm{e} 6))$;

$\mathrm{dc} 2=0.092 *(2 * 0.5 * 1.97 e-5 *((1870 * \mathrm{c} 0+21000 * \mathrm{c} 1) /(1870 * \mathrm{c} 0+21000 * \mathrm{c} 1+21396 * \mathrm{c} 5)) *(1-$ $\exp (-2.303 * 0.00133 *(1870 * c 0+21000 * c 1+21396 * c 5)))-\mathrm{k} 3 * c 0 * c 2-$

$\left.\mathrm{k} 4{ }^{*} \mathrm{c} 1{ }^{*} \mathrm{c} 2+\mathrm{k} 5{ }^{*} \mathrm{c} 0{ }^{*} \mathrm{c} 3+\mathrm{k} 6{ }^{*} \mathrm{c} 0{ }^{*} \mathrm{c} 4-\mathrm{k} 9{ }^{*} \mathrm{c} 2{ }^{*} \mathrm{c} 2-\mathrm{k} 11{ }^{*} \mathrm{c} 2{ }^{*} \mathrm{c} 3-\mathrm{k} 13{ }^{*} \mathrm{c} 2{ }^{*} \mathrm{c} 4-\mathrm{k} 14{ }^{*} \mathrm{c} 2{ }^{*} \mathrm{c} 5-\mathrm{k} 16 * \mathrm{c} 7{ }^{*} \mathrm{c} 2\right) ;$ $\mathrm{dc} 3=0.092 *\left(\mathrm{k} 3{ }^{*} \mathrm{c} 0{ }^{*} \mathrm{c} 2+\mathrm{k} 4{ }^{*} \mathrm{c} 2{ }^{*} \mathrm{c} 3-\mathrm{k} 5{ }^{*} \mathrm{c} 0{ }^{*} \mathrm{c} 3+\mathrm{k} 7{ }^{*} \mathrm{c} 4{ }^{*} 1 \mathrm{e}-6-\mathrm{k} 8{ }^{*} \mathrm{c} 3-\mathrm{k} 10{ }^{*} \mathrm{c} 3{ }^{*} \mathrm{c} 3-\mathrm{k} 11{ }^{*} \mathrm{c} 2{ }^{*} \mathrm{c} 3-\right.$ $\left.\mathrm{k} 12 * \mathrm{c} 3{ }^{*} \mathrm{c} 4\right)$;

$\mathrm{dc} 4=0.092 *\left(-\mathrm{k} 6{ }^{*} \mathrm{c} 0{ }^{*} \mathrm{c} 4-\mathrm{k} 7{ }^{*} \mathrm{c} 4{ }^{*} 1 \mathrm{e}-6+\mathrm{k} 8{ }^{*} \mathrm{c} 3-\mathrm{k} 12{ }^{*} \mathrm{c} 3{ }^{*} \mathrm{c} 4-\mathrm{k} 13{ }^{*} \mathrm{c} 2{ }^{*} \mathrm{c} 4\right)$;

$\mathrm{dc} 5=0.092 *\left(-\mathrm{k} 14{ }^{*} \mathrm{c} 2{ }^{*} \mathrm{c} 5\right) ;$

$\mathrm{dc} 6=0.092 *(\mathrm{k} 14 * \mathrm{c} 2 * \mathrm{c} 5) ;$

$\mathrm{dc} 7=0.092 *\left(\mathrm{k} 15{ }^{*} \mathrm{c} 6-\mathrm{k} 16{ }^{*} \mathrm{c} 7{ }^{*} \mathrm{c} 2\right)$;

o state deriv. vec.

$d x=[d c 0$ de1 de2 dc3 dc4 dc5 dc6 dc7 ]'; flag=0; new_data=[]; end 
PMAINDEG main calling file for the parameter estimation.

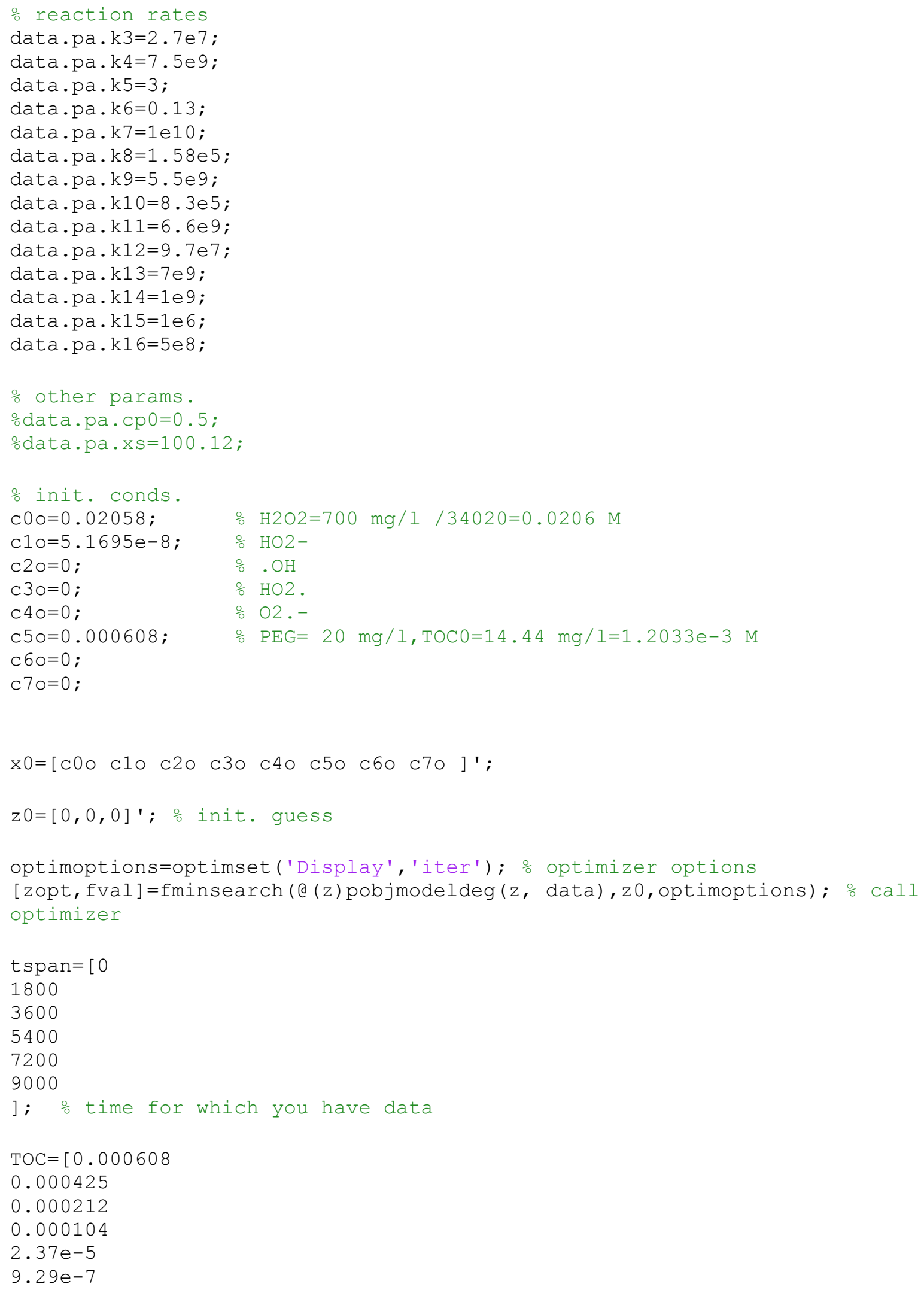


] i data points

options = odeset('RelTol', 1e-4, 'AbsTol', [1e-14 1e-14 1e-14 1 e-14 1 e-14 1 e-14 $1 e-141 e-14])$;

$[t, y]=\operatorname{ode15s}(@(t, x)$ pmodeldeg $(t, x, z o p t$, data $)$, tspan, $x 0$, options $)$; $\frac{\circ}{\text { integrate }}$ plot $(t / 60, y(:, 6) / 0.000608, '-. ')$; hold on;

plot (tspan/60, TOC/0.000608, 'o') ;

function $d x=$ pmodeldeg $(t, x, z$, data $)$ 
\% PMODELDEG dynamic model for degradation polymeric wastewater.

$\frac{o}{\circ}$ trans
$\mathrm{c} 0=\mathrm{x}(1) ;$
$\mathrm{c} 1=\mathrm{x}(2) ;$
$\mathrm{c} 2=\mathrm{x}(3) ;$
$\mathrm{C} 3=\mathrm{x}(4) ;$
$\mathrm{C} 4=\mathrm{x}(5) ;$
$\mathrm{C} 5=\mathrm{x}(6) ;$
$\mathrm{C} 6=\mathrm{x}(7) ;$
$\mathrm{C} 7=\mathrm{x}(8) ;$

$\circ \mathrm{qs} 1=\mathrm{x}(4)$;

o get params.

k3=data.pa.k3;

$\mathrm{k} 4=\mathrm{data} \cdot \mathrm{pa} \cdot \mathrm{k} 4$;

$\mathrm{k} 5=\mathrm{data} \cdot \mathrm{pa} \cdot \mathrm{k} 5$;

$\mathrm{k} 6=\mathrm{data} \cdot \mathrm{pa} \cdot \mathrm{k} 6$;

$\mathrm{k} 7=\mathrm{data} \cdot \mathrm{pa} \cdot \mathrm{k} 7$;

$\mathrm{k} 8=\mathrm{data} \cdot \mathrm{pa} \cdot \mathrm{k} 8$;

k9=data.pa.k9;

$\mathrm{k} 10=$ data $\cdot \mathrm{pa} \cdot \mathrm{k} 10$;

$\mathrm{k} 11=$ data $\cdot \mathrm{pa} \cdot \mathrm{k} 11$;

$\mathrm{k} 12=$ data $\cdot \mathrm{pa} \cdot \mathrm{k} 12$;

$\mathrm{k} 13=$ data $\cdot \mathrm{pa} \cdot \mathrm{k13}$;

$\mathrm{k} 14=\mathrm{data} \cdot \mathrm{pa} \cdot \mathrm{k} 14$ * $(1+\mathrm{z}(1))$;

$\mathrm{k} 15=$ data $\cdot \mathrm{pa} \cdot \mathrm{k} 15 *(1+\mathrm{z}(2))$;

$\mathrm{k} 16=\mathrm{data} \cdot \mathrm{pa} \cdot \mathrm{k} 15 *(1+\mathrm{z}(3))$;

\% model diff. eqs.

$\mathrm{dc} 0=0.092 *(-0.5 * 1.97 e-5 *((1870 * \mathrm{c} 0+21000 * \mathrm{c} 1) /(1870 * \mathrm{c} 0+21000 * \mathrm{c} 1+21396 * \mathrm{c} 5)) *(1-$ $\left.\exp \left(-2.303 * 0.00133 *\left(1870 * \mathrm{c} 0+21000 * \mathrm{c} 1+21396{ }^{*} \mathrm{c} 5\right)\right)\right)-\mathrm{k} 3{ }^{*} \mathrm{c} 0{ }^{*} \mathrm{c} 2-\mathrm{k} 4{ }^{*} \mathrm{c} 1{ }^{*} \mathrm{c} 2-\mathrm{k} 5{ }^{*} \mathrm{c} 0{ }^{*} \mathrm{c} 3-$ $\left.\mathrm{k} 6{ }^{*} \mathrm{c} 0 * \mathrm{c} 4+\mathrm{k} 9 * \mathrm{c} 2 * \mathrm{c} 2+\mathrm{k} 10 * \mathrm{c} 3 * \mathrm{c} 3+\mathrm{k} 12 * \mathrm{c} 3 * \mathrm{c} 4\right) *(1 /(1+10 \mathrm{e}-6))$;

$\mathrm{dc} 1=0.092 *(-0.5 * 1.97 e-5 *((1870 * \mathrm{c} 0+21000 * \mathrm{c} 1) /(1870 * \mathrm{c} 0+21000 * \mathrm{c} 1+21396 * \mathrm{c} 5)) *(1-$ $\exp (-2.303 * 0.00133 *(1870 * c 0+21000 * c 1+21396 * c 5)))-k 3 * c 0 * c 2-k 4 * c 1 * c 2-k 5 * c 0 * c 3-$ $\left.\mathrm{k} 6 * \mathrm{c} 0 * \mathrm{c} 4+\mathrm{k} 9{ }^{*} \mathrm{c} 2 * \mathrm{c} 2+\mathrm{k} 10 * \mathrm{c} 3 * \mathrm{c} 3+\mathrm{k} 12 * \mathrm{c} 3 * \mathrm{c} 4\right) *(1 /(1+10 \mathrm{e} 6))$;

$\mathrm{dc} 2=0.092 *(2 * 0.5 * 1.97 e-5 *((1870 * \mathrm{c} 0+21000 * \mathrm{c} 1) /(1870 * \mathrm{c} 0+21000 * \mathrm{c} 1+21396 * \mathrm{c} 5)) *(1-$ $\exp (-2.303 * 0.00133 *(1870 * c 0+21000 * c 1+21396 * c 5)))-\mathrm{k} 3 * c 0 * c 2-$

$\left.\mathrm{k} 4{ }^{*} \mathrm{c} 1{ }^{*} \mathrm{c} 2+\mathrm{k} 5{ }^{*} \mathrm{c} 0{ }^{*} \mathrm{c} 3+\mathrm{k} 6{ }^{*} \mathrm{c} 0{ }^{*} \mathrm{c} 4-\mathrm{k} 9{ }^{*} \mathrm{c} 2{ }^{*} \mathrm{c} 2-\mathrm{k} 11{ }^{*} \mathrm{c} 2{ }^{*} \mathrm{c} 3-\mathrm{k} 13{ }^{*} \mathrm{c} 2{ }^{*} \mathrm{c} 4-\mathrm{k} 14{ }^{*} \mathrm{c} 2{ }^{*} \mathrm{c} 5-\mathrm{k} 16{ }^{*} \mathrm{c} 7{ }^{*} \mathrm{c} 2\right) ;$ $\mathrm{dc} 3=0.092 *\left(\mathrm{k} 3{ }^{*} \mathrm{c} 0{ }^{*} \mathrm{c} 2+\mathrm{k} 4{ }^{*} \mathrm{c} 2{ }^{*} \mathrm{c} 3-\mathrm{k} 5{ }^{*} \mathrm{c} 0{ }^{*} \mathrm{c} 3+\mathrm{k} 7{ }^{*} \mathrm{c} 4{ }^{*} 1 e-6-\mathrm{k} 8{ }^{*} \mathrm{c} 3-\mathrm{k} 10{ }^{*} \mathrm{c} 3{ }^{*} \mathrm{c} 3-\mathrm{k} 11{ }^{*} \mathrm{c} 2{ }^{*} \mathrm{c} 3-\right.$ $\left.\mathrm{k} 12 * \mathrm{c} 3{ }^{*} \mathrm{c} 4\right)$;

$\mathrm{dc} 4=0.092 *\left(-\mathrm{k} 6{ }^{*} \mathrm{c} 0{ }^{*} \mathrm{c} 4-\mathrm{k} 7{ }^{*} \mathrm{c} 4{ }^{*} 1 e-6+\mathrm{k} 8{ }^{*} \mathrm{c} 3-\mathrm{k} 12{ }^{*} \mathrm{c} 3{ }^{*} \mathrm{c} 4-\mathrm{k} 13{ }^{*} \mathrm{c} 2{ }^{*} \mathrm{c} 4\right)$;

$\mathrm{dc} 5=0.092 *(-\mathrm{k} 14 * \mathrm{c} 2 * \mathrm{c} 5) ;$

$\mathrm{dc} 6=0.092 *\left(\mathrm{k} 14{ }^{*} \mathrm{c} 2{ }^{*} \mathrm{c} 5\right) ;$

$\mathrm{dc} 7=0.092 *\left(\mathrm{k} 15 \star^{\mathrm{c}} 6-\mathrm{k} 16{ }^{*} \mathrm{c} 7{ }^{*} \mathrm{c} 2\right)$;

o state deriv. vec.

$\mathrm{dx}=\left[\begin{array}{llllllll}\mathrm{de} 0 & \mathrm{dc} 1 & \mathrm{dc} & \mathrm{dc} 3 & \mathrm{dc} 4 & \mathrm{dc} 5 & \mathrm{dc} 6 & \mathrm{dc} 7\end{array}\right]^{\prime}$;

end 
ㅇ POBJMODELDEG objective function for the parameter estimation.

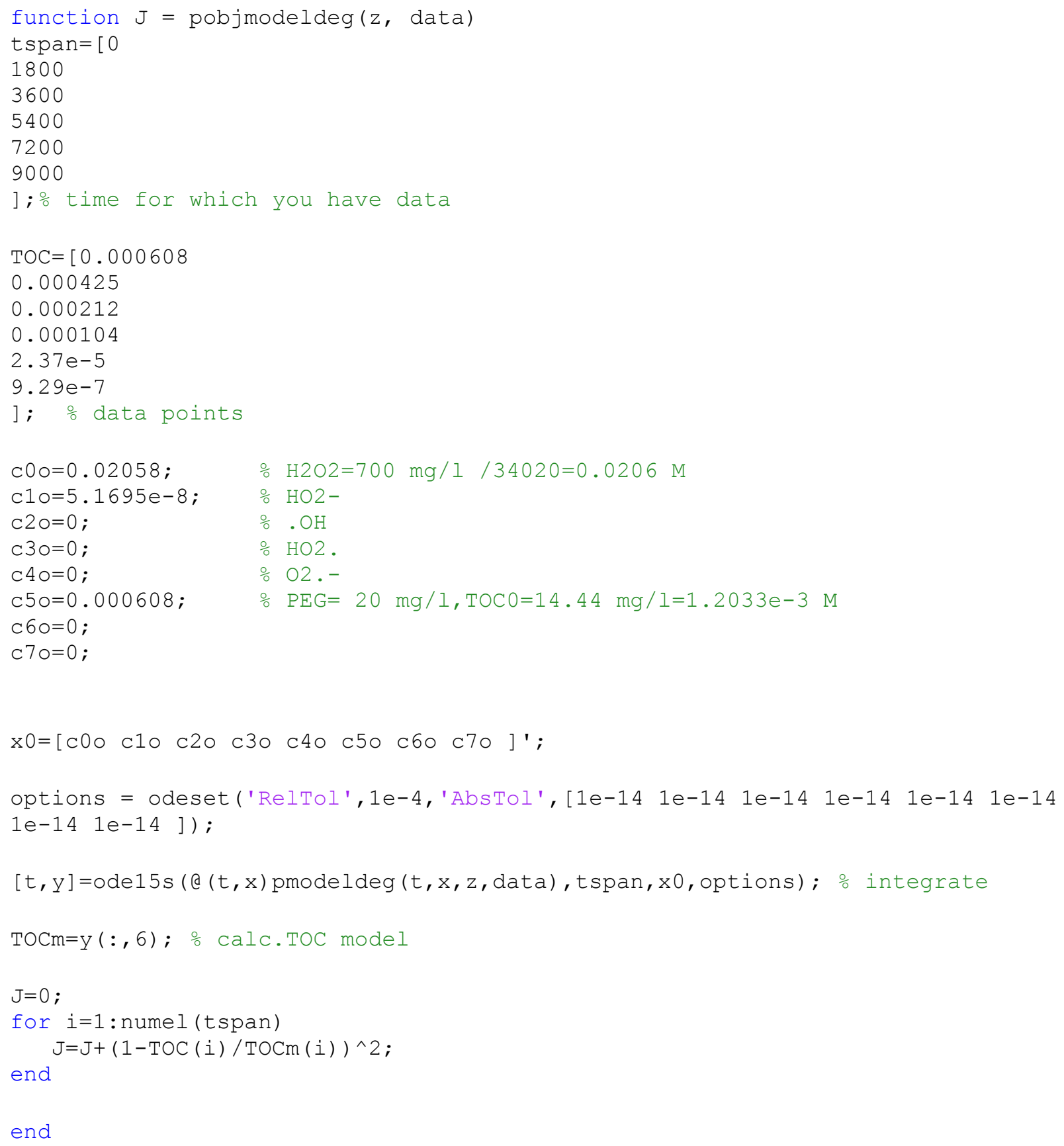

